Supporting Information for

\title{
Diastereodivergent Intermolecular 1,2-diamination of unactivated alkenes enabled by iodine catalysis
}

\author{
Satoshi Minakata*, Hayato Miwa, Kenya Yamamoto, Arata Hirayama, Sota Okumura \\ Department of Applied Chemistry, Graduate School of Engineering, Osaka University, \\ Yamadaoka 2-1, Suita, Osaka 565-0871, Japan.
}




\section{Table of Contents}

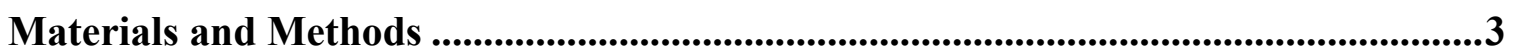

Supporting Imformation .................................................................................................................3

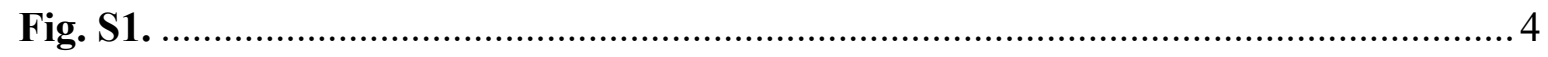

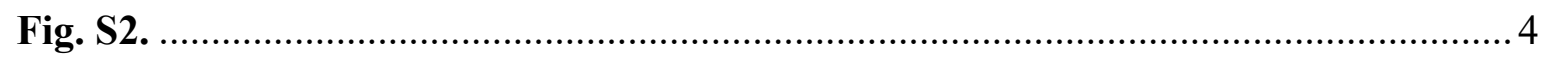

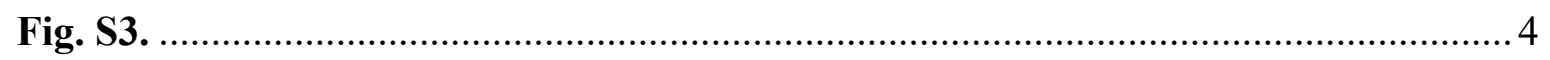

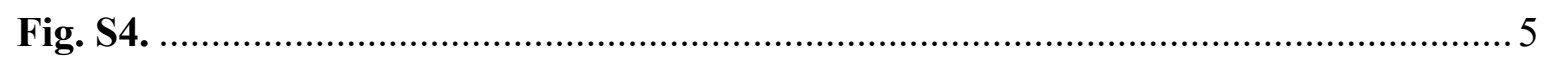

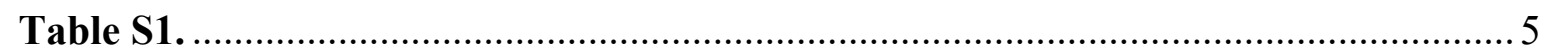

Plausible reaction pathway for anti-1,2-diamination of alkenes .....................................6

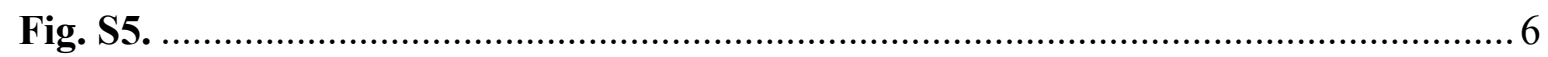

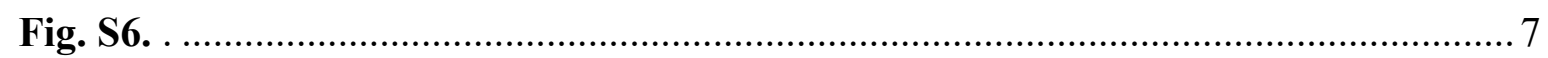

Plausible reaction pathway for $s y n-1,2$-diamination of alkenes ...................................8

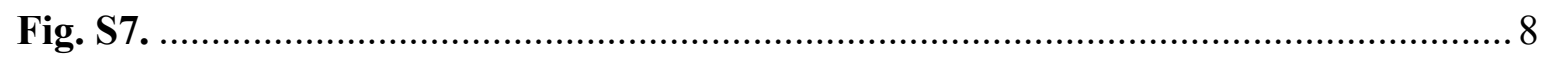

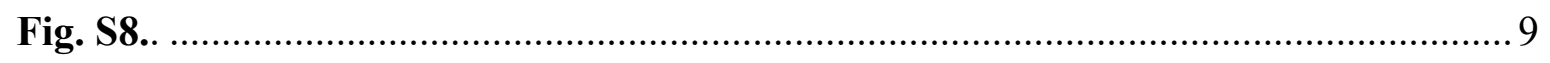

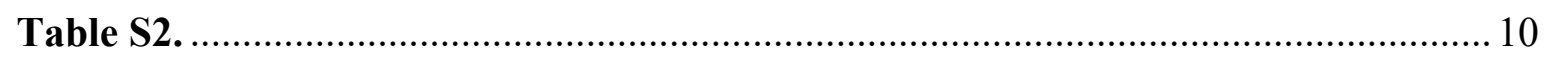

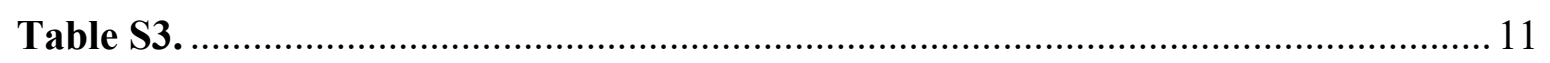

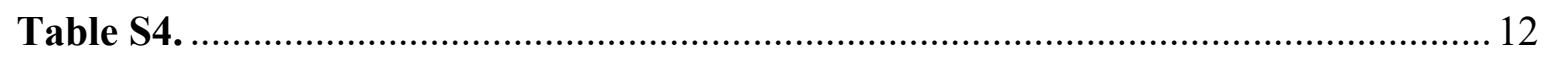

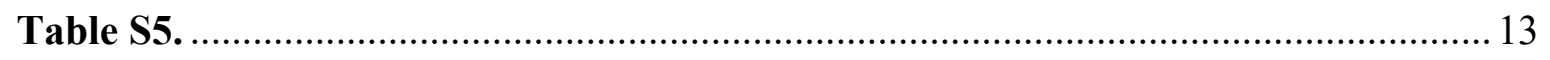

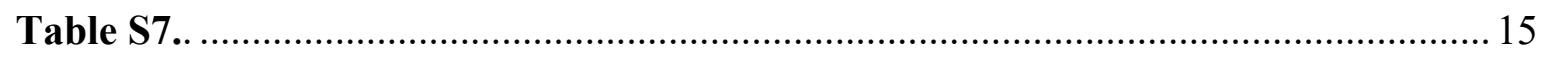

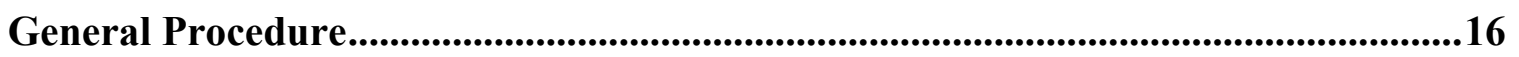

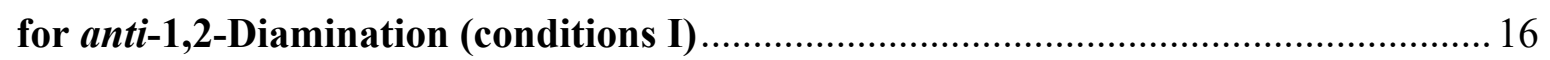

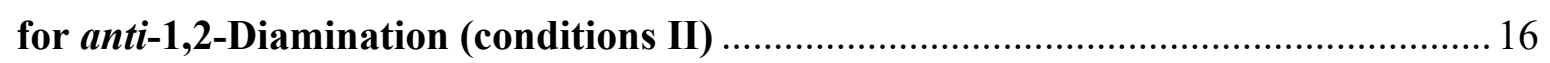

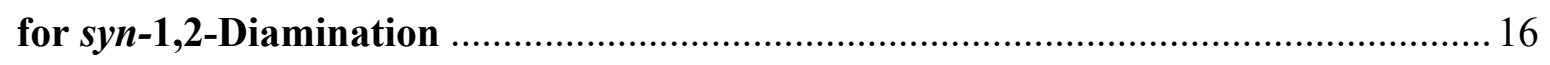

Experimental Procedures and Analytical Data ...........................................................17

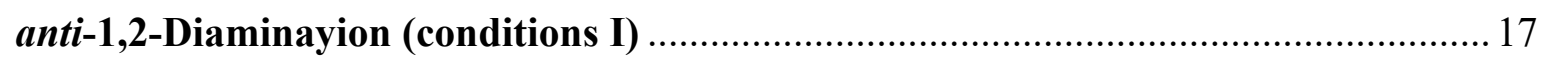

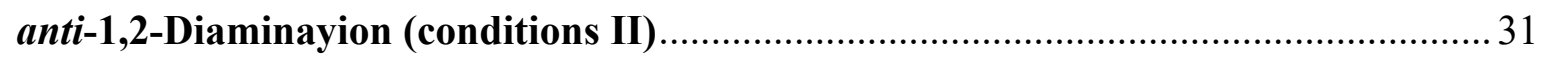

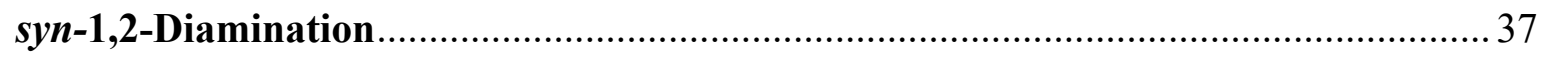

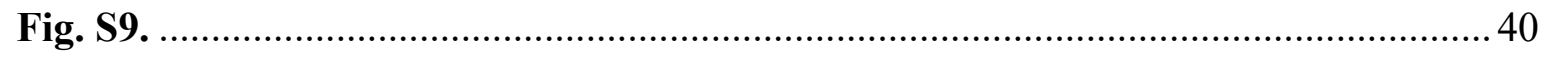

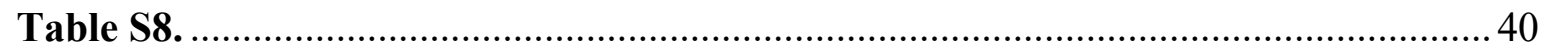

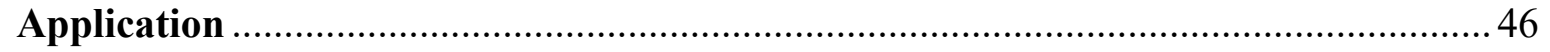

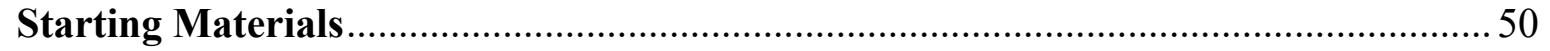

NMR Spectra ........................................................................................................................55

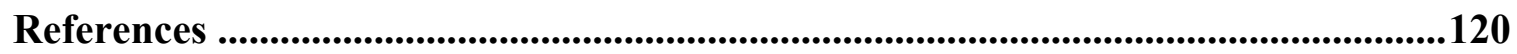




\section{Materials and Methods}

Unless otherwise noted, all reactions were performed in two-neck glass tubes under a nitrogen atmosphere. Sodium hypochlorite pentahydrate $\left(\mathrm{NaOCl} \cdot 5 \mathrm{H}_{2} \mathrm{O}\right)$ should be stored below $7{ }^{\circ} \mathrm{C}$. When weighing this reagent, you should take it out of the refrigerator immediately, weigh it, and use a plastic spatula. Concentration of solution was carried out by using a rotary evaporator and generally followed by removal of residual solvents on a vacuum line held at $0.1-1$ torr. Products were purified by chromatography on silica gel BW-300 (Fuji Silysia Chemical Ltd.) or recycling gel permeation chromatography (GPC) with JAI LC-908 using chloroform as an eluent. Commercial reagents and solvents were purchased from Sigma Aldrich, TCI, Wako Pure Chemical, nacalai tesque, Iharanikkei Chemical Industry and Kanto Chemical and used as received with the following exceptions: acetonitrile and tetrahydrofuran were used from a solvent purification system. Analytical thin-layer chromatography (TLC) was performed on pre-coated silica gel glass plates (Merck silica gel $60 \mathrm{~F}_{254}, 0.25 \mathrm{~mm}$ thickness). Compounds were visualized with UV lamp or treatment with an ethanolic solution of phosphomolybdic acid followed by heating or exposure to an iodine atmosphere. ${ }^{1} \mathrm{H},{ }^{13} \mathrm{C}$ and ${ }^{19} \mathrm{~F}$ NMR spectra were recorded on a JEOL JMTC-400/54/SS spectrometer ( ${ }^{1} \mathrm{H}$ NMR, $400 \mathrm{MHz} ;{ }^{13} \mathrm{C}$ NMR, 100 $\mathrm{MHz} ;{ }^{19} \mathrm{~F}$ NMR, $\left.377 \mathrm{MHz}\right) .{ }^{1} \mathrm{H}$ NMR chemical shifts for $\mathrm{CDCl}_{3}$ were determined relative to $\mathrm{Me}_{4} \mathrm{Si}(0.0 \mathrm{ppm})$ as an internal standard. In other cases, NMR spectra were calibrated using residual undeuterated solvent $\left({ }^{1} \mathrm{H}: \delta 2.50\right.$ for DMSO- $d_{6}, \delta 2.05$ for acetone- $d_{6} ;{ }^{13} \mathrm{C}: \delta 77.00$ for $\mathrm{CDCl}_{3}, \delta 39.50$ for DMSO- $d_{6}, \delta 29.80$ for acetone- $\left.d_{6}\right) .{ }^{19} \mathrm{~F}$ NMR chemical shifts were determined relative to $\mathrm{C}_{6} \mathrm{~F}_{6}(-162.5 \mathrm{ppm})$ as an external standard. Data are represented as follows: chemical shift, multiplicity $(\mathrm{br}=$ broad, $\mathrm{s}=$ singlet, $\mathrm{d}=$ doublet, $\mathrm{t}=$ triplet, $\mathrm{q}=$ quartet, $\mathrm{m}=$ multiplet), coupling constants in Hertz $(\mathrm{Hz})$. Infrared spectra were recorded on a SHIMADZU IRAffinity-1 FT-IR Spectrometer. High-resolution mass spectra were obtained on a JEOL JMS-DX303HF mass spectrometer. Melting points were determined on a Stanford Research Systems MPA100 OptiMelt Automated Melting Point System. X-ray crystal data were collected and processed using CrysAlisPro (Rigaku Oxford Diffraction).

Abbreviations: Ac - acetyl, BBS— $N, N$ '-bis(tert-butoxycarbonyl)sulfamide, Boc-tert-butyl carbamate, Bs-benzenesulfonyl, Cbz-benzyloxycarbonyl, $\mathrm{DIH}$-1,3-diiodo-5,5dimethylhydantoin, DMAP - N,N'-dimethyl-4-aminopyridine, DME-1,2-dimethoxyethane, DMF - $N, N$ '-dimethylformamide, DMSO - dimethylsulfoxide, EtOAc - ethyl acetate, $\mathrm{MeCN}$ - acetonitrile, $\mathrm{MeOH}$ - methanol, Ms - methanesulfonyl, Ns-2-nitrobenzenesulfonyl, $p$-Ns - 4-nitrobenzenesulfonyl, THF - tetrahydrofuran, Ts - $p$-toluenesulfonyl.

\section{Supporting Imformation}




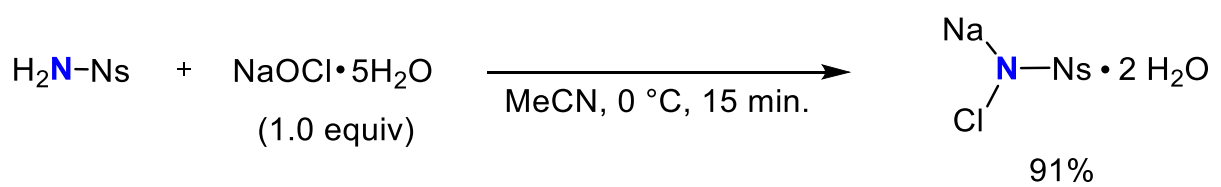

Fig. S1. Preparation of chloramine-Ns by the reaction of nosylamide with $\mathrm{NaOCl}$ petahydrate. (Experimental details were shown in the experimental procedure for synthesis of starting materials.)

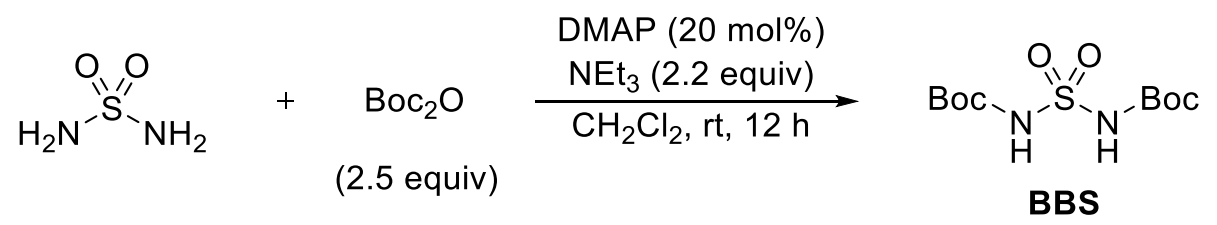

$71 \%$

Fig. S2. Preparation of $N, N^{\prime}$-bis(tert-butoxycarbonyl)sulfamide (BBS). (Experimental details were shown in the experimental procedure for synthesis of starting materials.)

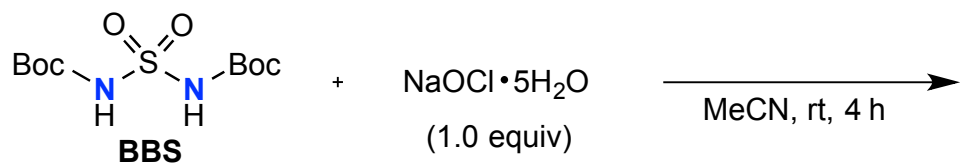

BBS

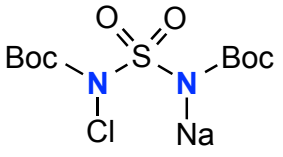

chloramine-BBS $96 \%$

Fig. S3. Preparation of chloramine-BBS by the reaction of BBS with $\mathrm{NaOCl}$ pentahydrate. (Experimental details were shown in the experimental procedure for synthesis of starting materials.) 

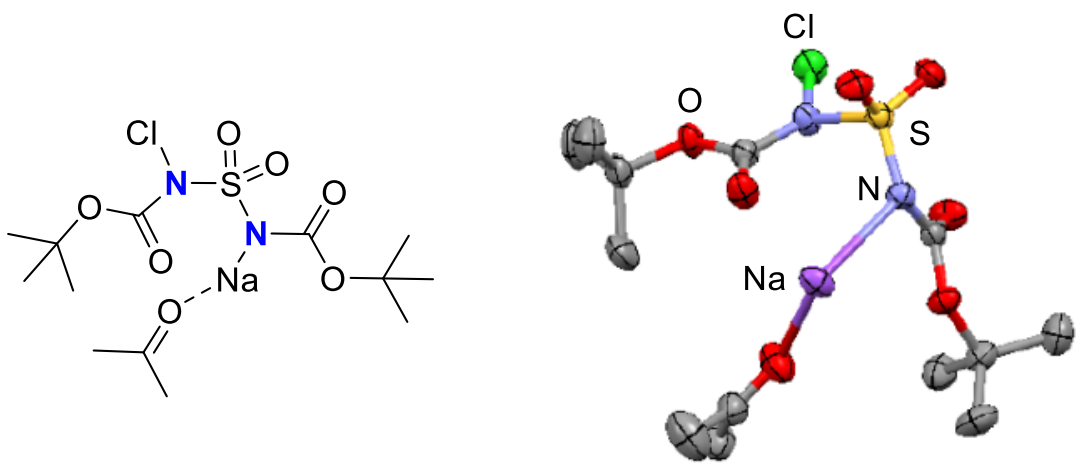

Fig. S4. Crystal structure of chloramine-BBS. CCDC 1919392 contains the supplementary crystallographic data. The data can be obtained free of charge from The Cambridge Crystallographic Data Centre via www.ccdc.cam.ac.uk/data_request/cif.

Table S1. Crystallographic data of chloramine-BBS

Empirical Formula

Formula Weight

Crystal System

Space Group

Unit cell dimensions

$V$

$Z$

Density (calculated)

Absorption coefficient

$\mathrm{R} 1(\mathrm{I}>2.00 \sigma(\mathrm{I}))$

wR2 (All reflections)

Crystal size

Goodness of Fit Indicator

No. of Reflections Measured
$\mathrm{C}_{13} \mathrm{H}_{24} \mathrm{ClN}_{2} \mathrm{NaO}_{7} \mathrm{~S}$

410.84

monoclinic

$\mathrm{P} 2{ }_{1} / \mathrm{c}$ (\#14)

$\mathrm{a}=9.96887(15) \AA$

$\mathrm{b}=17.1553(2) \AA$

$\mathrm{c}=11.82620(17) \AA$

$\beta=99.7876(14)^{\mathrm{O}}$

1993.07(5) $\AA^{3}$

4

$1.369 \mathrm{~g} / \mathrm{cm}^{3}$

$3.213 \mathrm{~cm}^{-1}$

0.0605

0.1862

$0.100 \times 0.050 \times 0.010 \mathrm{~mm}$

1.071

Total: 12324

Unique: $3952\left(\mathrm{R}_{\text {int }}=0.0322\right)$ 


\section{Plausible reaction pathway for anti-1,2-diamination of alkenes}

As depicted in Fig. S5, the reaction pathway was assumed based on our previous report. ${ }^{1}$ At first, iodine reacts with chloramine-Ns to afford $N$-chloro- $N$-iodosulfonamide. This iodo substituent attached to the nitrogen should act as an iodonium source to generate cyclic iodonium intermediate by the reaction with alkenes, subsequently nitrogen anion attacks this carbon to give this intermediate. Iodide of the liberated sodium iodide at initial step attacks the chlorine substituent, and the cyclization proceeds, leading to aziridine. The resulting $N$ nosylated aziridine should be readily ring-opened by another chloramine-Ns, leading to diaminated compounds with complete stereoselectivity. In order to clarify the ring opening step, the reaction of isolated N-Ns aziridine derived from trans-4-octene with nosylamide in $\mathrm{MeCN}$ at $80{ }^{\circ} \mathrm{C}$ for $12 \mathrm{~h}$ did not proceed at all, but the reaction with chloramine-Ns gave the desired diaminated compound. The same results were obtained in the case of the reaction with $\mathrm{BocNH}_{2}$.

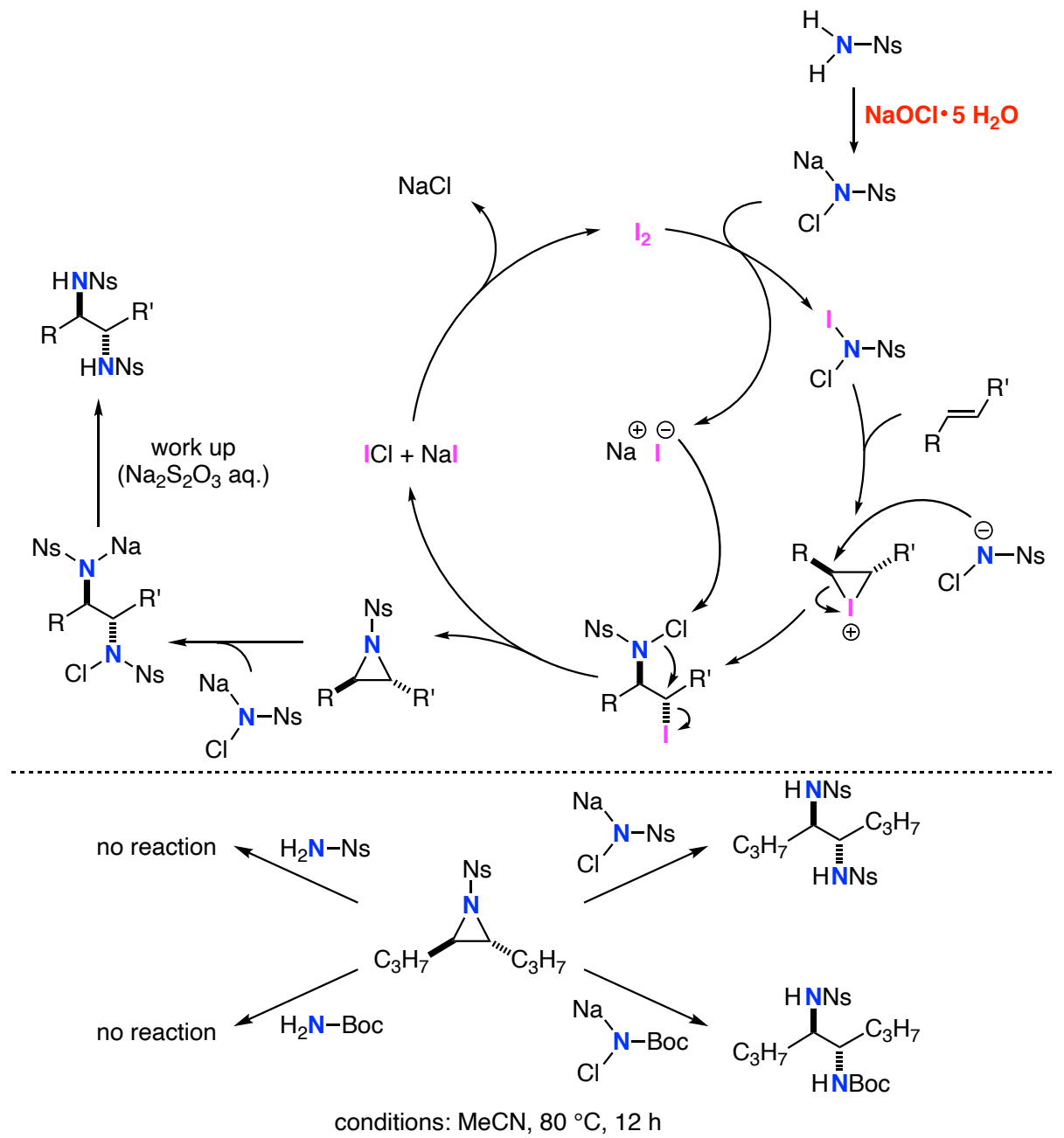

Fig. S5. Plausible reaction pathway for anti-1,2-diamination. 


$$
\underset{\mathrm{Cl}^{\prime}}{\mathrm{Na}-\mathrm{Ns} \cdot 2 \mathrm{H}_{2} \mathrm{O}} \frac{\mathrm{I}_{2}(1.0 \text { equiv })}{\mathrm{CD}_{3} \mathrm{CN}, \mathrm{rt}, 5 \mathrm{~min}} \underset{\mathrm{Cl}^{\prime}}{\stackrel{\mathrm{N}}{\mathrm{N}}-\mathrm{Ns}}
$$

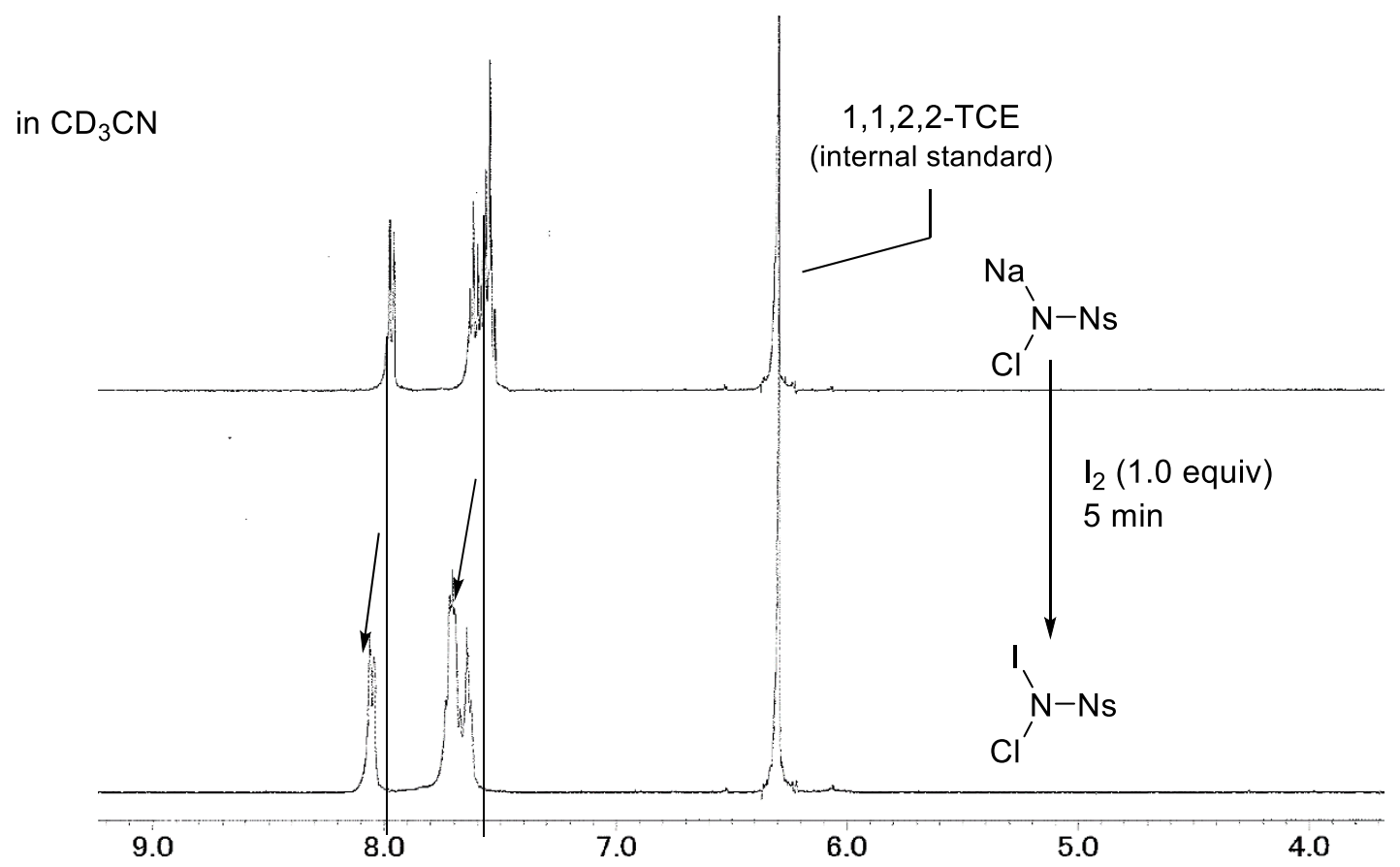

Fig. S6. Supporting experiments to clarify the reactive species for anti-1,2-diamination. 


\section{Plausible reaction pathway for syn-1,2-diamination of alkenes}

As depicted in Fig. S7, the reaction pathway was assumed based on our previous report. ${ }^{1}$ At first, iodine reacts with chloramine-BBS to afford $N$-chloro- $N$ '-iodosulfamide. This iodo substituent attached to the nitrogen should act as an iodonium source to generate cyclic iodonium intermediate by the reaction with alkenes, subsequently nitrogen anion attacks this carbon to give this intermediate. Iodide of the liberated sodium iodide at initial step attacks the chlorine substituent on the iodoaminated adduct, and the cyclization proceeds, leading to fivemembered ring with complete stereoselectivity.

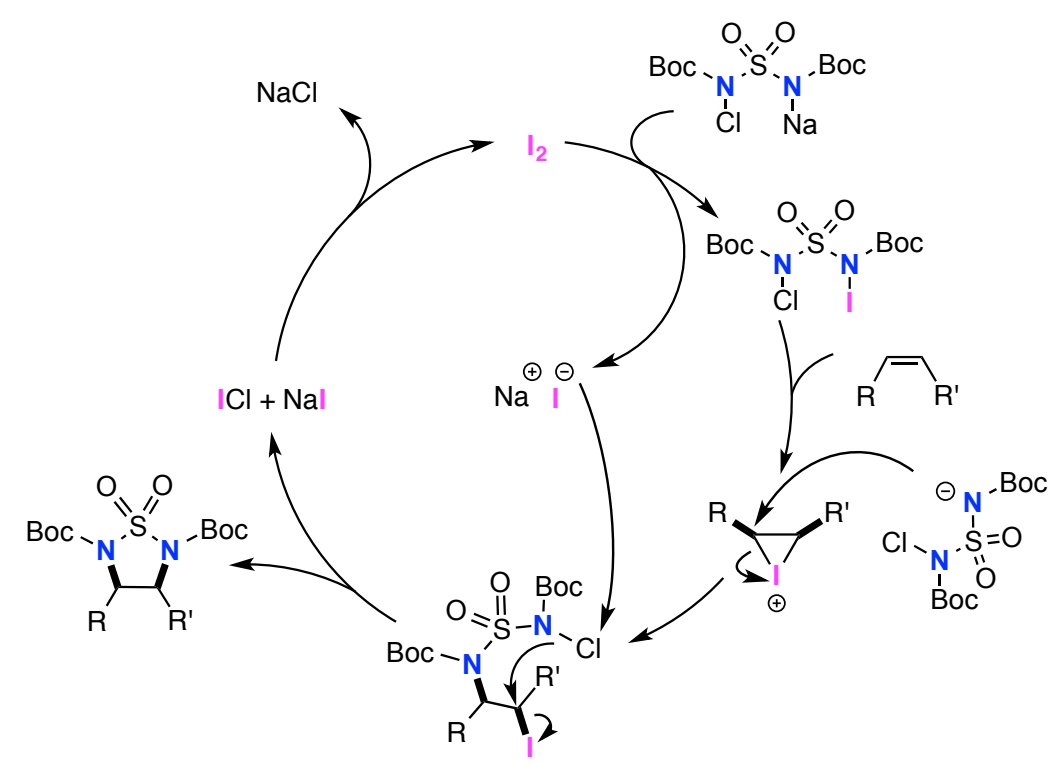

Fig. S7. Plausible reaction pathway for syn-1,2-diamination. 


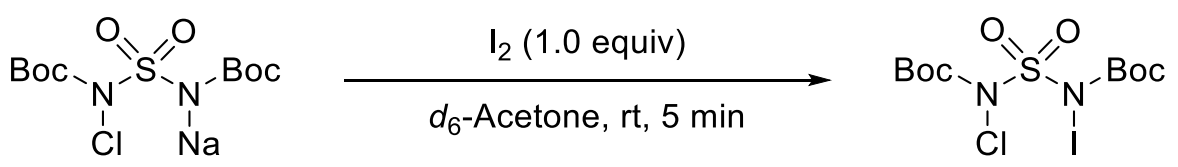

in $d_{6}$-Acetone

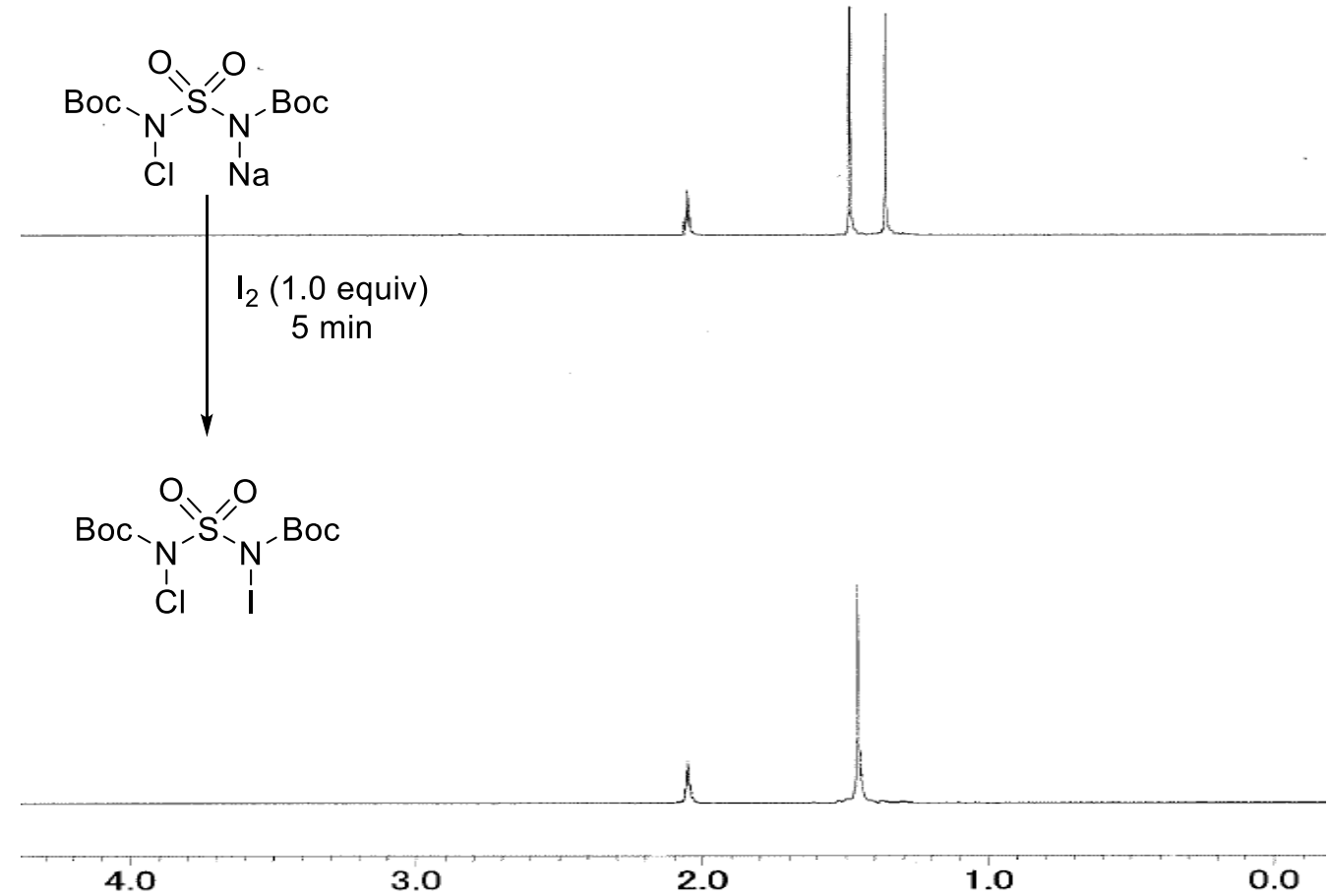

Fig. S8. Supporting experiments to clarify the reactive species for $s y n$-1,2-diamination. 
Table S2. $\mathrm{I}_{2}$-Catalyzed 1,2-diamination of an alkene with various chloramine salts.

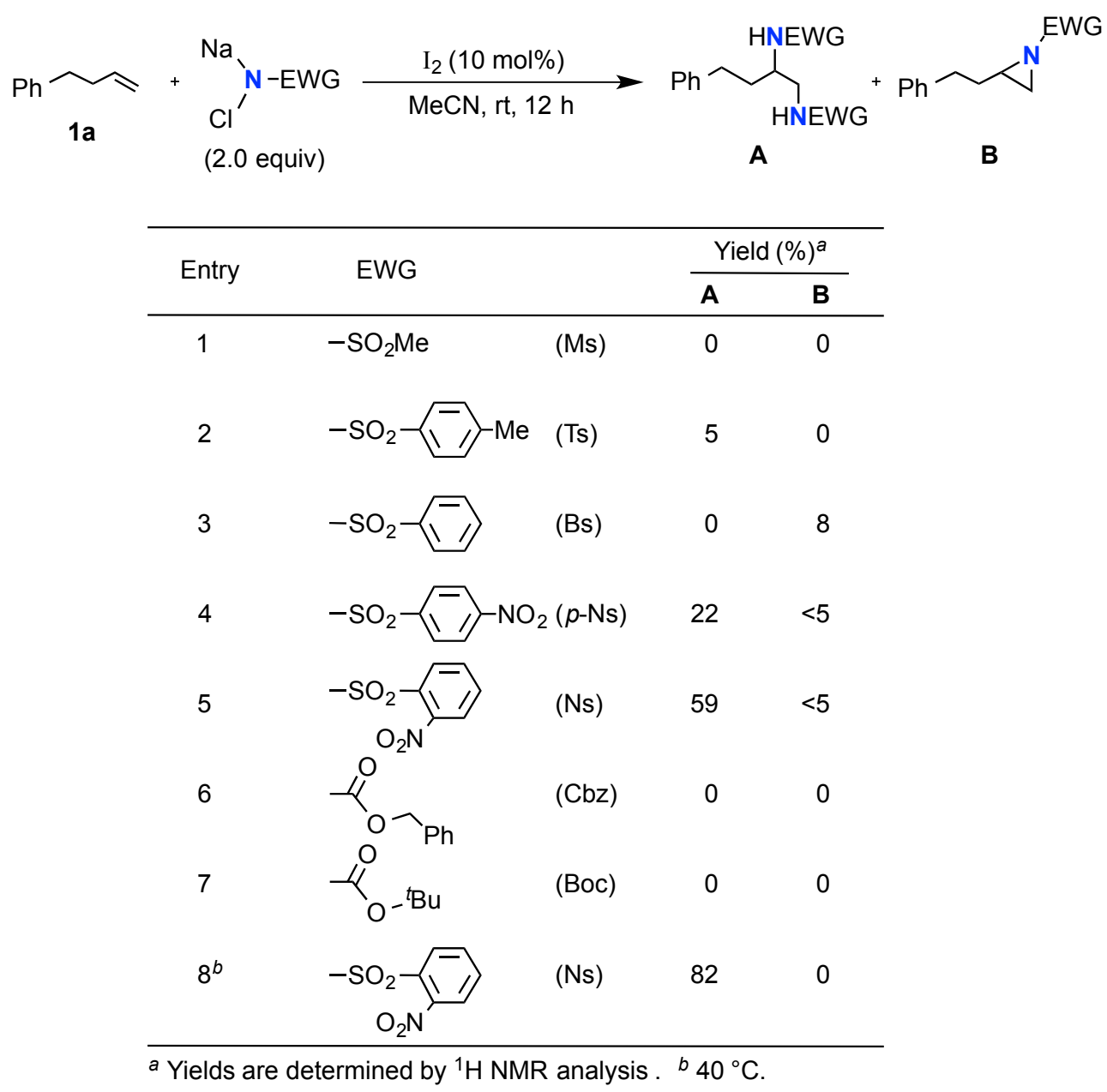


Table S3. $\mathrm{I}_{2}$-Catalyzed 1,2-diamination of an alkene with nosylamide in the presence of oxidants and/or base.

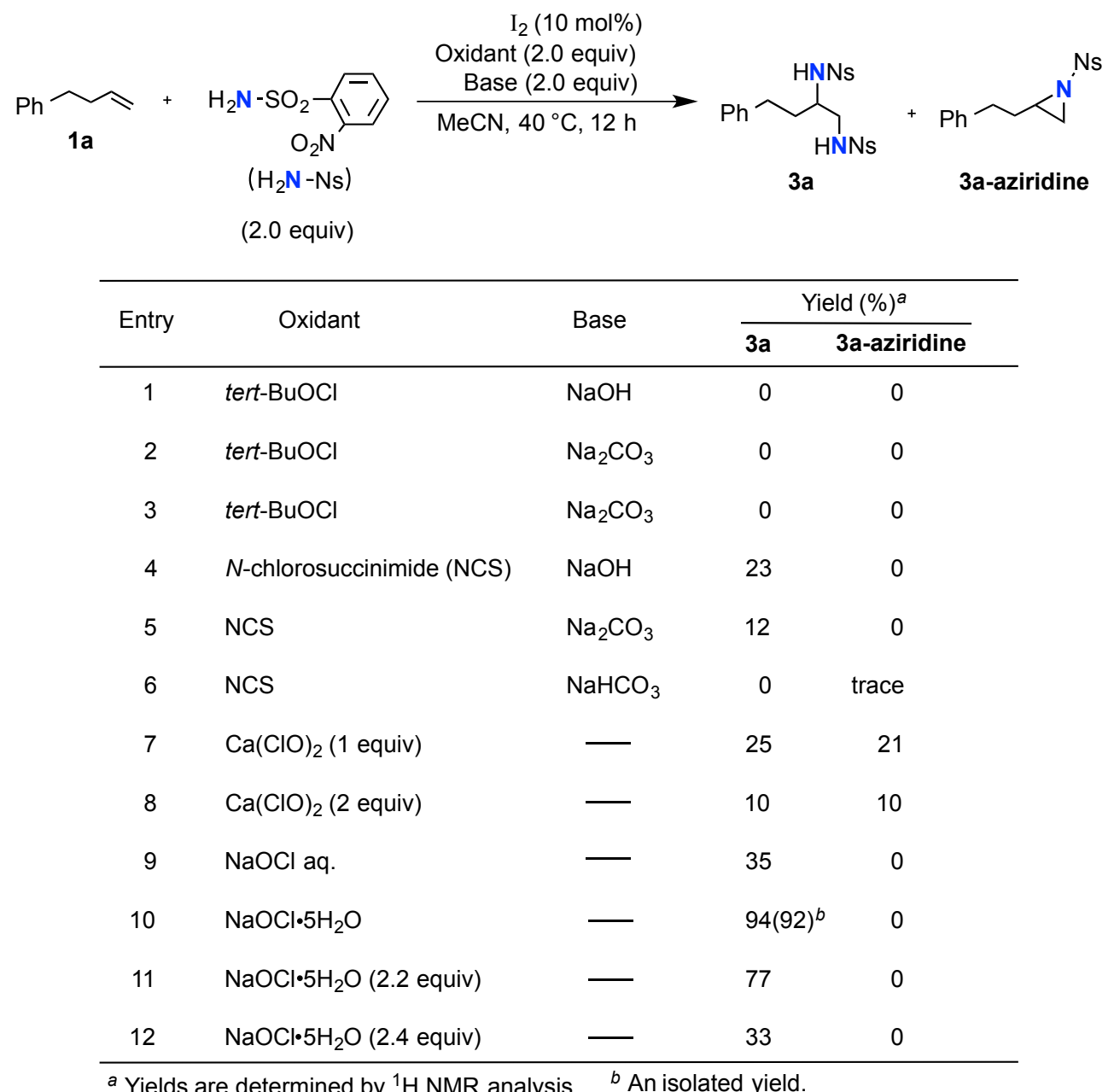


Table S4. Effect of solvents on the 1,2-diamination.

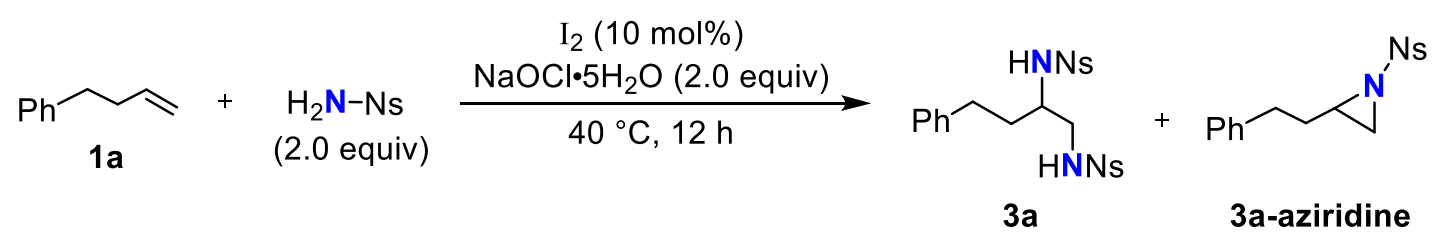

\begin{tabular}{|c|c|c|c|}
\hline \multirow{2}{*}{ Entry } & \multirow{2}{*}{ Solvent } & \multicolumn{2}{|c|}{ Yield $(\%)^{a}$} \\
\hline & & $3 a$ & 3a-aziridine \\
\hline 1 & $\mathrm{MeCN}$ & $94(92)^{b}$ & 0 \\
\hline 2 & THF & 78 & 5 \\
\hline 3 & DME & 26 & 0 \\
\hline 4 & EtOAc & 18 & 0 \\
\hline 5 & DMF & 15 & 0 \\
\hline 6 & $\mathrm{CH}_{2} \mathrm{Cl}_{2}$ & 0 & 0 \\
\hline 7 & toluene & 0 & 0 \\
\hline
\end{tabular}

${ }^{a}$ Yields are determined by ${ }^{1} \mathrm{H}$ NMR analysis.

${ }^{b} \mathrm{An}$ isolated yield. 
Table S5. Effects of temperature and amount of $I_{2}$ on the 1,2-diamination.

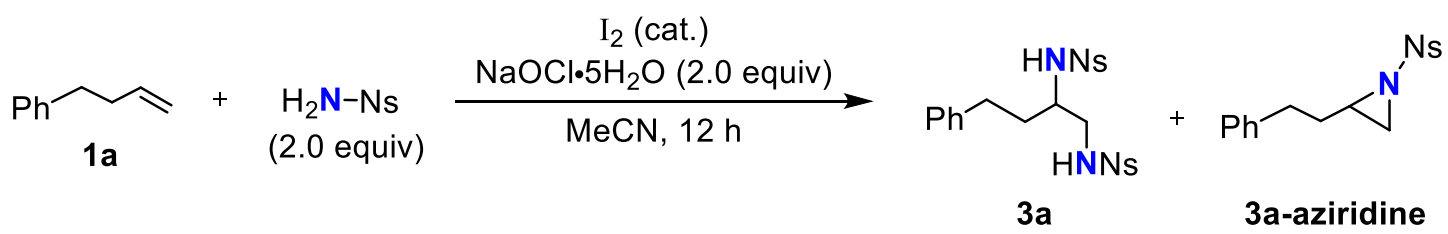

\begin{tabular}{|c|c|c|c|c|}
\hline \multirow{2}{*}{ Entry } & \multirow{2}{*}{$\mathrm{I}_{2}(\mathrm{~mol} \%)$} & \multirow{2}{*}{ Temp. $\left({ }^{\circ} \mathrm{C}\right)$} & \multicolumn{2}{|c|}{ Yield $(\%)^{a}$} \\
\hline & & & $3 \mathbf{a}$ & 3a-aziridine \\
\hline 1 & 10 & 30 & 82 & 0 \\
\hline 2 & 10 & 40 & $94(92)^{b}$ & 0 \\
\hline 3 & 10 & 50 & 82 & 0 \\
\hline 4 & 10 & 60 & 80 & 0 \\
\hline 5 & none & 40 & 0 & 0 \\
\hline 6 & 5 & 40 & 52 & 0 \\
\hline 7 & 20 & 40 & 84 & 0 \\
\hline
\end{tabular}

${ }^{a}$ Yields are determined by ${ }^{1} \mathrm{H}$ NMR analysis.

${ }^{b} \mathrm{An}$ isolated yield. 
Table S6. Solvent effects on 1,2-syn-diamination of cyclopentene.

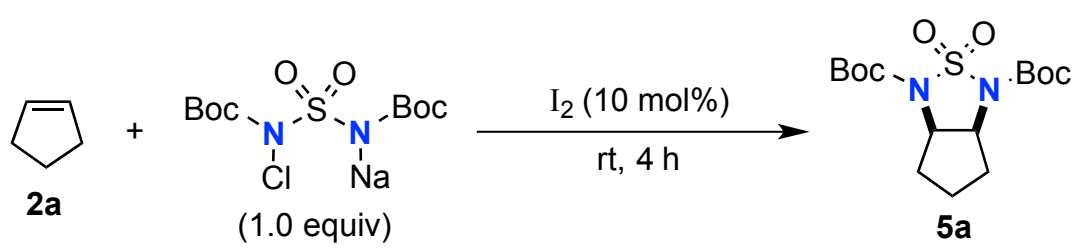

\begin{tabular}{ccr}
\hline Entry & Solvent & Yield $(\%)^{a}$ \\
\hline 1 & THF & 8 \\
2 & MeCN & trace \\
3 & MeOH & 2 \\
4 & Acetone & 14 \\
5 & DMSO & 0 \\
6 & DMF & 8 \\
7 & toluene & 5 \\
8 & DME & 22 \\
9 & 1.4-dioxane & 5 \\
10 & MTBE & 10 \\
11 & MCH & 5 \\
12 & Et $_{2} \mathrm{O}$ & 25 \\
\hline
\end{tabular}

a Yields are determined by ${ }^{1} \mathrm{H}$ NMR analysis. 
Table S7. Optimization of reaction conditions for 1,2-syn-diamination of cyclopentene.

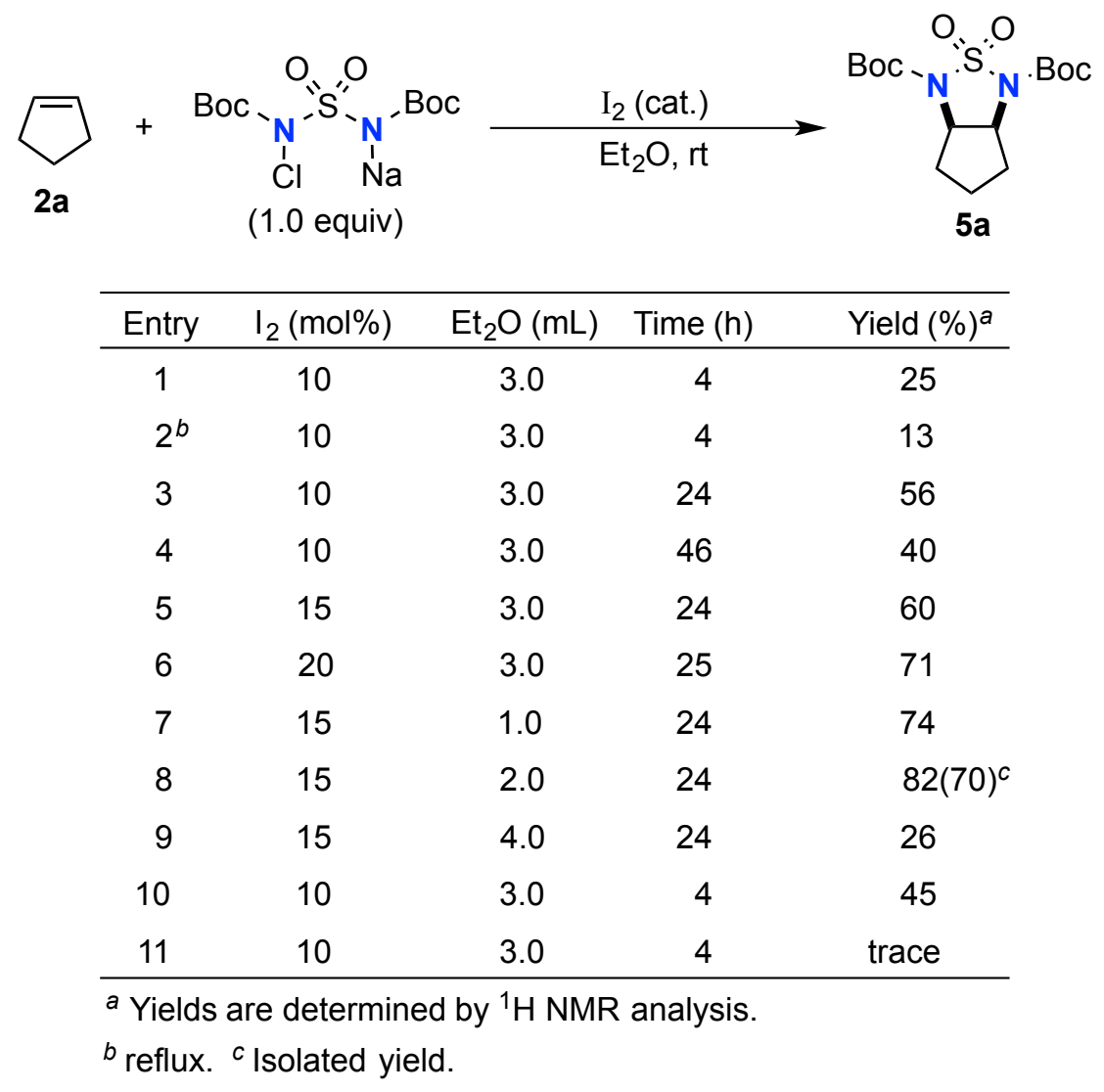




\section{General Procedure}

\section{for anti-1,2-Diamination (conditions I)}

Sodium hypochlorite pentahydrate $(165.0 \mathrm{mg}, 1.00 \mathrm{mmol})$ was added to a stirred solution of the alkene ( $0.5 \mathrm{mmol}, 1.0$ equiv), 2-nitrobenzenesulfonamide (204.0 $\mathrm{mg}, 1.01 \mathrm{mmol})$ and iodine $(12.7 \mathrm{mg}, 0.05 \mathrm{mmol}, 10 \mathrm{~mol} \%)$ at room temperature under a nitrogen atmosphere in $\mathrm{MeCN}(3 \mathrm{~mL})$. The reaction mixture was warmed to $40{ }^{\circ} \mathrm{C}$ and stirred for $12 \mathrm{~h}$, unless otherwise stated. The reaction was quenched by adding an aqueous $1 \mathrm{M}$ sodium thiosulfate solution (5 $\mathrm{mL})$ and the aqueous layer was extracted with EtOAc or $\mathrm{CH}_{2} \mathrm{Cl}_{2}(3 \times 30 \mathrm{~mL})$. The combined organic extracts were dried over anhydrous $\mathrm{Na}_{2} \mathrm{SO}_{4}$ and concentrated in vacuo. The crude product was purified by flash column chromatography on silica gel and/or by washing with $\mathrm{Et}_{2} \mathrm{O}$.

\section{for anti-1,2-Diamination (conditions II)}

Sodium hypochlorite pentahydrate $(181.7 \mathrm{mg}, 1.10 \mathrm{mmol}, 2.2$ equiv) was added to a stirred solution of the alkene ( $0.5 \mathrm{mmol}, 1.0$ equiv), 2-nitrobenzenesulfonamide ( $223 \mathrm{mg}, 1.10 \mathrm{mmol}$ ) and iodine (12.7 mg, $0.05 \mathrm{mmol}, 10 \mathrm{~mol} \%)$ at room temperature under a nitrogen atmosphere in $\mathrm{MeCN}(3 \mathrm{~mL})$. The reaction mixture was then stirred at room temperature for $12 \mathrm{~h}$, then it warmed to $80^{\circ} \mathrm{C}$ and again stirred for $12 \mathrm{~h}$, unless otherwise stated. The reaction was quenched by adding a $1 \mathrm{M}$ solution of sodium thiosulfate aqueous $(5 \mathrm{~mL})$ and aqueous layer was extracted with EtOAc $(3 \times 30 \mathrm{~mL})$. The combined organic extracts were dried over anhydrous $\mathrm{Na}_{2} \mathrm{SO}_{4}$ and concentrated in vacuo. The crude product was purified by flash column chromatography on silica gel and/or by washing with $\mathrm{Et}_{2} \mathrm{O}$.

\section{for syn-1,2-Diamination}

The alkene ( $0.5 \mathrm{mmol}, 1.0$ equiv) was added to a stirred solution of chloramine-BBS (177.3 $\mathrm{mg}, 0.50 \mathrm{mmol})$ and iodine $(19.1 \mathrm{mg}, 0.075 \mathrm{mmol}, 15 \mathrm{~mol} \%)$ in ether $(2.0 \mathrm{~mL})$ at room temperature under a nitrogen atmosphere. The reaction mixture was stirred under the conditions described in each experimental procedure for syn-1,2-diamination (Fig. 3). The reaction was quenched by adding a $1 \mathrm{M}$ solution of sodium thiosulfate aqueous $(2.0 \mathrm{~mL})$ and the aqueous layer was extracted with $\mathrm{CH}_{2} \mathrm{Cl}_{2}(3 \times 30 \mathrm{~mL})$. The combined organic extracts were dried over anhydrous $\mathrm{Na}_{2} \mathrm{SO}_{4}$ and concentrated in vacuo. The crude product was purified by flash column chromatography on silica gel. 


\section{Experimental Procedures and Analytical Data}

anti-1,2-Diaminayion (conditions I)

$N, N^{\prime}$-(4-phenylbutane-1,2-diyl)bis(2-nitrobenzenesulfonamide) (3a)

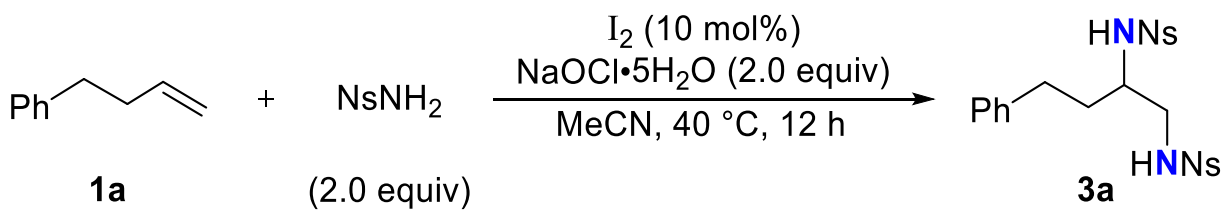

Following the general procedure (conditions I), 4-phenyl-1-butene (1a: $64.4 \mathrm{mg}, 0.49 \mathrm{mmol}$ ), iodine (12.9 mg, $0.05 \mathrm{mmol}$ ), 2-nitrobenzenesulfonamide (204.0 mg, $1.01 \mathrm{mmol}$ ) and sodium hypochlorite pentahydrate $(165.0 \mathrm{mg}, 1.00 \mathrm{mmol})$ were stirred at $40{ }^{\circ} \mathrm{C}$ in $\mathrm{MeCN}$ for $12 \mathrm{~h}$. Purification by flash column chromatography on silica gel $\left(\mathrm{CH}_{2} \mathrm{Cl}_{2} / \mathrm{MeOH}=19: 1\right)$ gave the product (3a: $239.0 \mathrm{mg}, 92 \%$ ) as a white solid. $\mathrm{mp}: 152.5-153.7{ }^{\circ} \mathrm{C}$; TLC: $\mathrm{R}_{\mathrm{f}}=0.40$ (hexane/EtOAc = 3:2); ${ }^{1} \mathrm{H}$ NMR $\left(400 \mathrm{MHz}, \mathrm{CDCl}_{3}\right) \delta 8.10-8.00(\mathrm{~m}, 2 \mathrm{H}), 7.92-7.82(\mathrm{~m}, 2 \mathrm{H})$, 7.80-7.67 (m, 4H), 7.30-7.11 (m, 3H), 6.99 (d, $J=6.8 \mathrm{~Hz}, 2 \mathrm{H}), 5.56(\mathrm{dd}, J=6.4,6.4 \mathrm{~Hz}, 1 \mathrm{H})$, $5.41(\mathrm{~d}, J=7.2 \mathrm{~Hz}, 1 \mathrm{H}), 3.63-3.53(\mathrm{~m}, 1 \mathrm{H}), 3.29-3.11(\mathrm{~m}, 2 \mathrm{H}), 2.69-2.49(\mathrm{~m}, 1 \mathrm{H}), 2.55-2.43$ $(\mathrm{m}, 1 \mathrm{H}), 2.01-1.89(\mathrm{~m}, 1 \mathrm{H}), 1.81-1.68(\mathrm{~m}, 1 \mathrm{H}) ;{ }^{13} \mathrm{C} \mathrm{NMR}\left(100 \mathrm{MHz}, \mathrm{CDCl}_{3}\right) \delta 147.9,147.7$, $140.1,133.9,133.8,133.1,132.9,130.9,130.7,128.5,128.2,126.2,125.5,54.5,47.6,34.2$, 31.4 (Three $\mathrm{sp}^{2}$ signals were not observed because of overlapping.); IR (ATR) 3353, 3348, 3102, 3095, 2956, 2924, 1594, 1533, 1444, 1419, 1409, 1360, 1343, 1311, 1164, 1125, 1091, 1059, 854, 816, $790 \mathrm{~cm}^{-1}$; HRMS (FAB+): $m / z$ calcd. for $\mathrm{C}_{22} \mathrm{H}_{23} \mathrm{~N}_{4} \mathrm{O}_{8} \mathrm{~S}_{2}\left([\mathrm{M}+\mathrm{H}]^{+}\right)$535.0957, found 535.0961 .

$N, N^{\prime}$-(3-phenylpropane-1,2-diyl)bis(2-nitrobenzenesulfonamide) (3b)

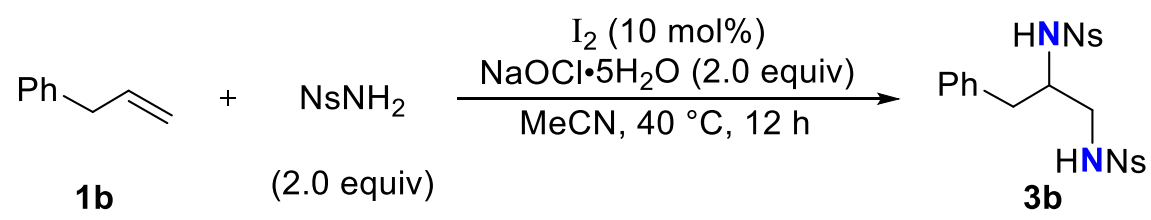

Following the general procedure (conditions I), allylbenzene (1b: $57.3 \mathrm{mg}, 0.48 \mathrm{mmol}$ ), iodine (13.1 mg, $0.05 \mathrm{mmol})$, 2-nitrobenzenesulfonamide (203.0 $\mathrm{mg}, 1.00 \mathrm{mmol}$ ) and sodium hypochlorite pentahydrate $(165.8 \mathrm{mg}, 1.01 \mathrm{mmol})$ were stirred at $40{ }^{\circ} \mathrm{C}$ in $\mathrm{MeCN}$ for $12 \mathrm{~h}$. Washing the crude product with $\mathrm{Et}_{2} \mathrm{O}$ gave the product (3b: $161.3 \mathrm{mg}, 64 \%$ ) as a white solid. 
mp: $187.5-188.1{ }^{\circ} \mathrm{C}$; TLC: $\mathrm{R}_{\mathrm{f}}=0.40$ (hexane/EtOAc $\left.=1: 1\right) ;{ }^{1} \mathrm{H}$ NMR (400 MHz, DMSO- $\left.d_{6}\right)$ $\delta 8.24$ (brs, 1H), 8.08 (brs, 1H), 8.03-8.00 (m, 1H), 7.99-7.94 (m, 1H), 7.94-7.84 (m, 2H), $7.78(\mathrm{~d}, J=8.0 \mathrm{~Hz}, 1 \mathrm{H}), 7.67$ (ddd, $J=2.0,8.0,8.0 \mathrm{~Hz}, 1 \mathrm{H}), 7.59-7.50(\mathrm{~m}, 2 \mathrm{H}), 7.02-6.82$ (m, 5H), 3.58 (brs, 1H), 3.11-3.01 (m, 1H), 3.00-2.90 (m, 1H), $2.86(\mathrm{dd}, J=4.0,13.6 \mathrm{~Hz}, 1 \mathrm{H})$ (One $\mathrm{C}\left(\mathrm{sp}^{3}\right)-\mathrm{H}$ signal is overlapped with residual DMSO signal.); ${ }^{13} \mathrm{C} \mathrm{NMR}(100 \mathrm{MHz}$, DMSO- $\left.d_{6}\right) \delta 147.7,146.7,137.4,134.3,133.6,133.2$, 132.8, 132.6, 132.4, 129.7, 129.3, 129.0, 127.9, 126.2, 124.7, 124.5, 55.7, 47.3, 36.9; IR (ATR) 3329, 3268, 3262, 3105, 3097, 2930, 2879, 1592, 1545, 1417, 1362, 1346, 1327, 1293, 1163, 1125, 1086, 1059, 1044, 976, 795, 731 $\mathrm{cm}^{-1}$; HRMS (FAB+): $m / z$ calcd. for $\mathrm{C}_{21} \mathrm{H}_{21} \mathrm{~N}_{4} \mathrm{O}_{8} \mathrm{~S}_{2}\left([\mathrm{M}+\mathrm{H}]^{+}\right)$521.0801, found 521.0795.

\section{$N, N^{\prime}$-(octane-1,2-diyl)bis(2-nitrobenzenesulfonamide) (3c)}

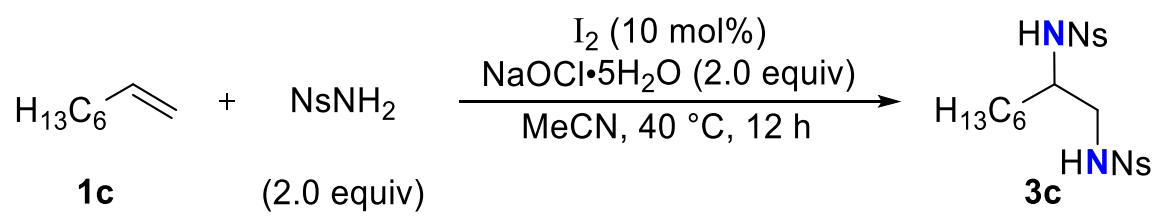

Following the general procedure (conditions I), 1-octene (1c: $56.3 \mathrm{mg}, 0.50 \mathrm{mmol}$ ), iodine (13.2 mg, $0.05 \mathrm{mmol}$ ), 2-nitrobenzenesulfonamide (203.4 $\mathrm{mg}, 1.01 \mathrm{mmol}$ ) and sodium hypochlorite pentahydrate $(166.4 \mathrm{mg}, 1.01 \mathrm{mmol})$ were stirred at $40{ }^{\circ} \mathrm{C}$ in $\mathrm{MeCN}$ for $12 \mathrm{~h}$. Washing the crude product with $\mathrm{Et}_{2} \mathrm{O}$ gave the product (3c: $176.5 \mathrm{mg}, 69 \%$ ) as a white solid. mp: 156.5-157.2 ${ }^{\circ} \mathrm{C}$; TLC: $\mathrm{R}_{\mathrm{f}}=0.43$ (hexane/EtOAc $\left.=1: 1\right) ;{ }^{1} \mathrm{H}$ NMR (400 MHz, DMSO- $\left.d_{6}\right)$ $\delta 8.09(\mathrm{dd}, J=6.0,6.0 \mathrm{~Hz}, 1 \mathrm{H}), 8.05-7.90(\mathrm{~m}, 5 \mathrm{H}), 7.90-7.79(\mathrm{~m}, 4 \mathrm{H}), 3.35-3.19(\mathrm{~m}, 1 \mathrm{H})$, 2.97-2.79 (m, 2H), 1.52-1.38 (m, 1H), 1.30-1.17 (m, 1H), 1.16-1.02 (m, 3H), 1.02-0.81 (m, $5 \mathrm{H}), 0.77(\mathrm{t}, J=7.2 \mathrm{~Hz}, 3 \mathrm{H}) ;{ }^{13} \mathrm{C} \mathrm{NMR}\left(100 \mathrm{MHz}, \mathrm{DMSO}-d_{6}\right) \delta 147.6,147.1,134.2,134.1$, $133.3,132.7,132.53,132.47,129.9,129.5,124.6,124.3,53.2,46.9,31.0,30.8,28.0,24.5,21.8$, 13.9; IR (ATR) 3353, 3101, 2959, 2927, 2859, 1592, 1534, 1444, 1439, 1419, 1346, 1303, $1171,1162,1143,1129,1122,1105,1059,982,967,855,812,785,742,727 \mathrm{~cm}^{-1}$; HRMS $(\mathrm{FAB}+): m / z$ calcd. for $\mathrm{C}_{20} \mathrm{H}_{27} \mathrm{~N}_{4} \mathrm{O}_{8} \mathrm{~S}_{2}\left([\mathrm{M}+\mathrm{H}]^{+}\right)$515.1265, found 515.1263.

\section{$N, N^{\prime}$-(7-bromoheptane-1,2-diyl)bis(2-nitrobenzenesulfonamide) (3d)}

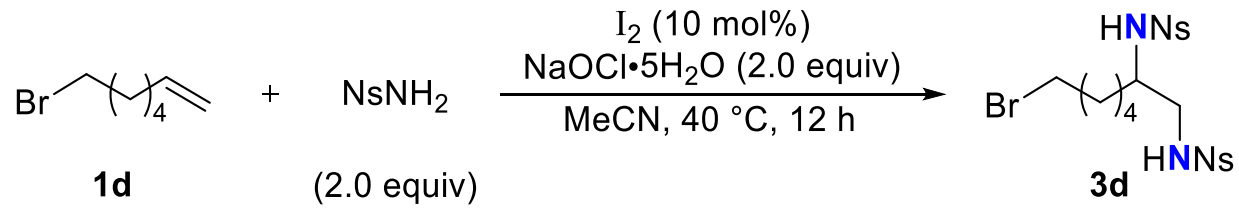


Following the general procedure (conditions I), 7-bromo-1-heptene (1d: $89.0 \mathrm{mg}, 0.50 \mathrm{mmol}$ ), iodine (13.6 mg, $0.05 \mathrm{mmol}$ ), 2-nitrobenzenesulfonamide (202.6 mg, $1.00 \mathrm{mmol}$ ) and sodium hypochlorite pentahydrate $(165.2 \mathrm{mg}, 1.00 \mathrm{mmol})$ were stirred at $40{ }^{\circ} \mathrm{C}$ in $\mathrm{MeCN}$ for $12 \mathrm{~h}$. Purification by flash column chromatography on silica gel (hexane/EtOAc $=7: 3$ ) gave the product (3d: $174.2 \mathrm{mg}, 60 \%$ ) as a white solid. $\mathrm{mp}: 212.0-213.8{ }^{\circ} \mathrm{C}$; $\mathrm{TLC}: \mathrm{R}_{\mathrm{f}}=0.38$ (hexane/EtOAc = 1:1); ${ }^{1} \mathrm{H}$ NMR $\left(400 \mathrm{MHz}, \mathrm{CDCl}_{3}\right) \delta 8.18-8.11(\mathrm{~m}, 1 \mathrm{H}), 8.10-8.03(\mathrm{~m}, 1 \mathrm{H})$, $7.90-7.81(\mathrm{~m}, 2 \mathrm{H}), 7.80-7.69(\mathrm{~m}, 4 \mathrm{H}), 5.64(\mathrm{dd}, J=6.4,6.4 \mathrm{~Hz}, 1 \mathrm{H}), 5.44(\mathrm{~d}, J=7.6 \mathrm{~Hz}, 1 \mathrm{H})$, $3.64-3.54(\mathrm{~m}, 1 \mathrm{H}), 3.29(\mathrm{t}, J=6.0 \mathrm{~Hz}, 2 \mathrm{H}), 3.28-3.10(\mathrm{~m}, 2 \mathrm{H}), 1.77-1.54(\mathrm{~m}, 3 \mathrm{H}), 1.52-1.39$ $(\mathrm{m}, 1 \mathrm{H}), 1.33-1.21(\mathrm{~m}, 3 \mathrm{H}), 1.20-1.06(\mathrm{~m}, 1 \mathrm{H}) ;{ }^{13} \mathrm{C} \mathrm{NMR}\left(100 \mathrm{MHz}, \mathrm{CDCl}_{3}\right) \delta 147.9,147.6$, 134.0, $133.85133 .81,133.15,133.10,133.0,130.9,130.7,125.5,54.9,47.7,33.5,32.4,32.2$, 27.4, 24.4 (One sp ${ }^{2}$ signal was not observed because of overlapping.); IR (ATR) 3348, 3335, 3100, 3022, 2941, 2862, 2261, 1964, 1852, 1594, 1534, 1441, 1417, 1346, 1304, 1270, 1163, $1125,1098,1059,1022,963,911,853 \mathrm{~cm}^{-1}$; HRMS (FAB+): $m / z$ calcd. for $\mathrm{C}_{19} \mathrm{H}_{24} \mathrm{BrN}_{4} \mathrm{O}_{8} \mathrm{~S}_{2}$ $\left([\mathrm{M}+\mathrm{H}]^{+}\right) 579.0219$, found 579.0214 .

\section{$4 N, N^{\prime}$-(methyl undecanoate-10,11-diyl)bis(2-nitrobenzenesulfonamide) (3e)}

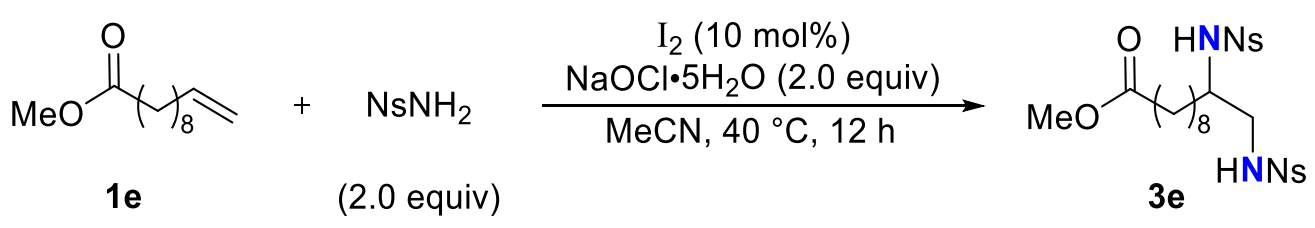

Following the general procedure (conditions I), methyl 10-undecenoate (1e: $99.5 \mathrm{mg}, 0.50$ $\mathrm{mmol}$ ), iodine (13.6 mg, $0.05 \mathrm{mmol})$, 2-nitrobenzenesulfonamide (202.7 mg, $1.00 \mathrm{mmol}$ ) and sodium hypochlorite pentahydrate $(164.3 \mathrm{mg}, 1.00 \mathrm{mmol})$ were stirred at $40{ }^{\circ} \mathrm{C}$ in $\mathrm{MeCN}$ for $12 \mathrm{~h}$. Purification by flash column chromatography on silica gel (hexane/EtOAc $=4: 1$ ) gave the product (3e: $244.8 \mathrm{mg}, 81 \%$ ) as a white solid. $\mathrm{mp}: 103.2-104.0{ }^{\circ} \mathrm{C}$; TLC: $\mathrm{R}_{\mathrm{f}}=0.34$ (hexane/EtOAc = 1:1); ${ }^{1} \mathrm{H}$ NMR $\left(400 \mathrm{MHz}, \mathrm{CDCl}_{3}\right) \delta 8.17-8.07(\mathrm{~m}, 2 \mathrm{H}), 7.92-7.84(\mathrm{~m}, 2 \mathrm{H})$, $7.79-7.73(\mathrm{~m}, 4 \mathrm{H}), 5.59$ (dd, $J=6.4,6.4 \mathrm{~Hz}, 1 \mathrm{H}), 5.33$ (d, $J=7.2 \mathrm{~Hz}, 1 \mathrm{H}), 3.67(\mathrm{~s}, 3 \mathrm{H}), 3.60$ $3.49(\mathrm{~m}, 1 \mathrm{H}), 3.26-3.11(\mathrm{~m}, 2 \mathrm{H}), 2.29(\mathrm{t}, J=7.6 \mathrm{~Hz}, 2 \mathrm{H}) 1.63-1.53(\mathrm{~m}, 3 \mathrm{H}), 1.49-1.37(\mathrm{~m}$, 1H), 1.29-1.01 (m, 10H); $\left.{ }^{13} \mathrm{C} \mathrm{NMR} \mathrm{(100} \mathrm{MHz,} \mathrm{CDCl}_{3}\right) \delta 174.3,148.0,147.7,134.0,133.80$, $133.78,133.2$, 133.1, 132.9, 131.0, 130.7, 125.5, 55.0, 51.5, 47.9, 34.0, 32.5, 28.98 28.93, 28.8, 25.2, 24.8 (One $\mathrm{sp}^{2}$ and one $\mathrm{sp}^{3}$ signals were not observed because of overlapping.); IR (ATR) 3348, 2934, 2854, 1717, 1593, 1533, 1457, 1442, 1418, 1346, 1306, 1255, 1231, 1198, 1164 , $1113,1061 \mathrm{~cm}^{-1}$; $\quad$ HRMS (FAB+): $m / z$ calcd. for $\mathrm{C}_{24} \mathrm{H}_{33} \mathrm{~N}_{4} \mathrm{O}_{10} \mathrm{~S}_{2}\left([\mathrm{M}+\mathrm{H}]^{+}\right) 601.1638$, found 601.1646 . 


\section{$N, N^{\prime}$-(3-hydroxypropane-1,2-diyl)bis(2-nitrobenzenesulfonamide) (3f)}

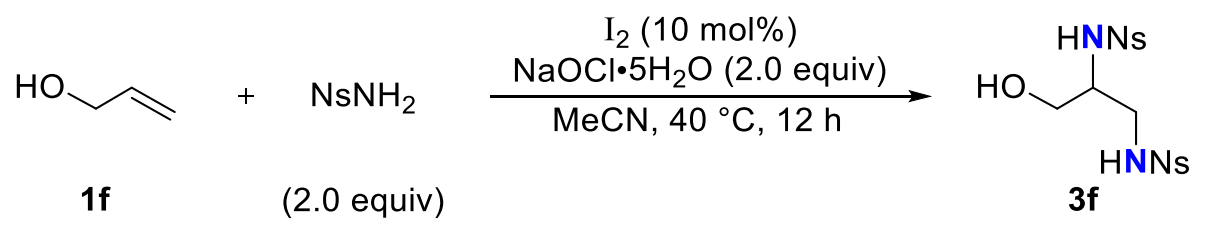

Following the general procedure (conditions I), allyl alcohol (1f: $29.0 \mathrm{mg}, 0.50 \mathrm{mmol}$ ), iodine (13.4 mg, $0.05 \mathrm{mmol}$ ), 2-nitrobenzenesulfonamide (203.4 $\mathrm{mg}, 1.01 \mathrm{mmol}$ ) and sodium hypochlorite pentahydrate $(167.1 \mathrm{mg}, 1.02 \mathrm{mmol})$ were stirred at $40{ }^{\circ} \mathrm{C}$ in $\mathrm{MeCN}$ for $12 \mathrm{~h}$. Purification by flash column chromatography on silica gel (hexane/EtOAc $=1: 1$ ) gave the product (3f: $173.8 \mathrm{mg}, 75 \%$ ) as a white solid. $\mathrm{mp}: 61.6-62.4{ }^{\circ} \mathrm{C}$; $\mathrm{TLC}: \mathrm{R}_{\mathrm{f}}=0.10$ (hexane/EtOAc = 1:1); ${ }^{1} \mathrm{H}$ NMR $\left(400 \mathrm{MHz}, \mathrm{CDCl}_{3}\right) \delta 8.19-8.12(\mathrm{~m}, 1 \mathrm{H}), 8.10-8.06(\mathrm{~m}, 1 \mathrm{H})$, $7.92-7.85(\mathrm{~m}, 2 \mathrm{H}), 7.82-7.71(\mathrm{~m}, 4 \mathrm{H}), 5.94(\mathrm{~d}, J=7.6 \mathrm{~Hz}, 1 \mathrm{H}), 5.79(\mathrm{dd}, J=5.6,5.6 \mathrm{~Hz}, 1 \mathrm{H})$, 3.88-3.78 (m, 1H), 3.75-3.61 (m, 2H), 3.39-3.21 (m, 2H), 2.15 (brs, $1 \mathrm{H}) ;{ }^{13} \mathrm{C}$ NMR $(100 \mathrm{MHz}$, $\left.\mathrm{CDCl}_{3}\right) \delta 147.9,147.7,134.0,133.2,133.1,132.9,131.0,130.7,125.7,125.6,62.1,55.1,44.6$ (Two $\mathrm{sp}^{2}$ signals were not observed because of overlapping.); IR (ATR) 3542, 3324, 3102, 2952, 2881, 1560, 1539, 1533, 1506, 1440, 1344, 1161, 1123, 1060, 968, 854, 782, $739 \mathrm{~cm}^{-1}$; HRMS (FAB+): $m / z$ calcd. for $\mathrm{C}_{15} \mathrm{H}_{17} \mathrm{~N}_{4} \mathrm{O}_{9} \mathrm{~S}_{2}\left([\mathrm{M}+\mathrm{H}]^{+}\right)$461.0437, found 461.0435 .

\section{$N, N^{\prime}$-(7-hydroxyheptane-1,2-diyl)bis(2-nitrobenzenesulfonamide) (3g)}

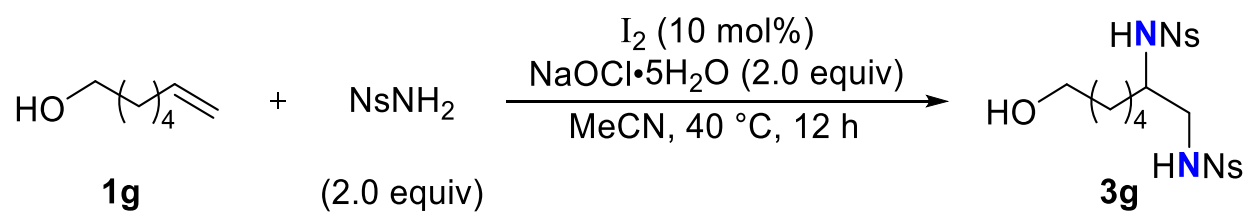

Following the general procedure (conditions I), 6-hepten-1-ol (1g: $56.5 \mathrm{mg}, 0.50 \mathrm{mmol}$ ), iodine (13.3 mg, $0.05 \mathrm{mmol})$, 2-nitrobenzenesulfonamide (203.4 $\mathrm{mg}, 1.01 \mathrm{mmol}$ ) and sodium hypochlorite pentahydrate $(163.5 \mathrm{mg}, 0.99 \mathrm{mmol})$ were stirred at $40{ }^{\circ} \mathrm{C}$ in $\mathrm{MeCN}$ for $12 \mathrm{~h}$. Purification by flash column chromatography on silica gel (hexane/EtOAc $=1: 1$ ) gave the product (3g: $225.5 \mathrm{mg}, 96 \%)$ as a white solid. mp: 65.1-65.9 ${ }^{\circ} \mathrm{C}$; $\mathrm{TLC}: \mathrm{R}_{\mathrm{f}}=0.10$ (hexane/EtOAc $=1: 1) ;{ }^{1} \mathrm{H}$ NMR $\left(400 \mathrm{MHz}, \mathrm{CDCl}_{3}\right) \delta 8.23-8.03(\mathrm{~m}, 2 \mathrm{H}), 7.95-7.65(\mathrm{~m}, 6 \mathrm{H})$, $5.64(\mathrm{dd}, J=6.4,6.4 \mathrm{~Hz}, 1 \mathrm{H}), 5.44(\mathrm{~d}, J=7.6 \mathrm{~Hz}, 1 \mathrm{H}), 3.65-3.45(\mathrm{~m}, 3 \mathrm{H}), 3.28-3.10(\mathrm{~m}, 2 \mathrm{H})$, 2.75-1.10 (m, 9H); ${ }^{13} \mathrm{C}$ NMR (100 MHz, $\left.\mathrm{CDCl}_{3}\right) \delta 147.9,147.6,134.0,133.8,133.1,132.9$, 130,9 130.7, 125.4, 62.4, 54.9, 47.7, 32.4, 32.0, 25.0, 24.9 (Three sp ${ }^{2}$ signals were not observed because of overlapping.); IR (ATR) 3348, 3101, 2940, 2859, 1593, 1534, 1419, 1344, 1162, 
1125, 1059, 854, 811, 782, 742, $729 \mathrm{~cm}^{-1}$; HRMS $(\mathrm{CI}+): \mathrm{m} / z$ calcd. for $\mathrm{C}_{19} \mathrm{H}_{25} \mathrm{~N}_{4} \mathrm{O}_{9} \mathrm{~S}_{2}$ $\left([\mathrm{M}+\mathrm{H}]^{+}\right) 517.1063$, found 517.1066.

\section{$N, N^{\prime}$-(undecanal-10,11-diyl)bis(2-nitrobenzenesulfonamide) (3h)}

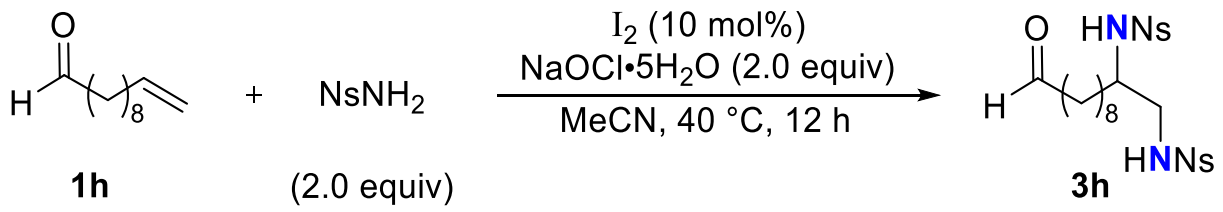

Following the general procedure (conditions I), 10-undecenal (1 h: $82.4 \mathrm{mg}, 0.49 \mathrm{mmol}$ ), iodine (13.2 mg, $0.05 \mathrm{mmol})$, 2-nitrobenzenesulfonamide (203.0 $\mathrm{mg}, 1.00 \mathrm{mmol}$ ) and sodium hypochlorite pentahydrate $(164.9 \mathrm{mg}, 1.00 \mathrm{mmol})$ were stirred at $40{ }^{\circ} \mathrm{C}$ in $\mathrm{MeCN}$ for $12 \mathrm{~h}$. Purification by flash column chromatography on silica gel (hexane/EtOAc $=7: 3$ ) gave the product (3h: $197.4 \mathrm{mg}, 71 \%$ ) as a colorless oil. TLC: $\mathrm{R}_{\mathrm{f}}=0.28$ (hexane/ EtOAc $\left.=1: 1\right) ;{ }^{1} \mathrm{H}$ NMR $\left(400 \mathrm{MHz}, \mathrm{CDCl}_{3}\right) \delta 9.77(\mathrm{t}, J=2.0 \mathrm{~Hz}, 1 \mathrm{H}), 8.18-8.12(\mathrm{~m}, 1 \mathrm{H}), 8.11-8.07(\mathrm{~m}, 1 \mathrm{H})$, $7.92-7.84(\mathrm{~m}, 2 \mathrm{H}), 7.80-7.72(\mathrm{~m}, 4 \mathrm{H}), 5.58(\mathrm{dd}, J=5.6,5.6 \mathrm{~Hz}, 1 \mathrm{H}), 5.31(\mathrm{~d}, J=7.6 \mathrm{~Hz}, 1 \mathrm{H})$, $3.62-3.50(\mathrm{~m}, 1 \mathrm{H}), 3.27-3.12(\mathrm{~m}, 2 \mathrm{H}), 2.42(\mathrm{dt}, J=2.0,7.2 \mathrm{~Hz}, 2 \mathrm{H}) 1.65-1.52(\mathrm{~m}, 3 \mathrm{H}), 1.48-$ $1.38(\mathrm{~m}, 1 \mathrm{H}), 1.38-1.05(\mathrm{~m}, 10 \mathrm{H}) ;{ }^{13} \mathrm{C} \mathrm{NMR}\left(100 \mathrm{MHz}, \mathrm{CDCl}_{3}\right) \delta 202.9,148.0,147.7,134.0$, 133.8, 133.2, 133.1, 132.9, 131.0, 130.7, 125.55, 125.52, 55.0, 47.9, 43.8, 32.6, 29.0, 28.9, 28.8, 25.1, 21.9 (One $\mathrm{sp}^{2}$ and one $\mathrm{sp}^{3}$ signals were not observed because of overlapping.); IR (ATR) 3314, 2928, 2855, 1717, 1537, 1418, 1346, 1165, 1124, 1059, 854, 783, $741 \mathrm{~cm}^{-1}$; HRMS $(\mathrm{FAB}+): m / z$ calcd. for $\mathrm{C}_{23} \mathrm{H}_{31} \mathrm{~N}_{4} \mathrm{O}_{9} \mathrm{~S}_{2}\left([\mathrm{M}+\mathrm{H}]^{+}\right)$571.1532, found 571.1526.

\section{$N, N^{\prime}$-(1-(2-oxopyrrolidin-1-yl)ethane-1,2-diyl)bis(2-nitrobenzenesulfonamide) (3i)}

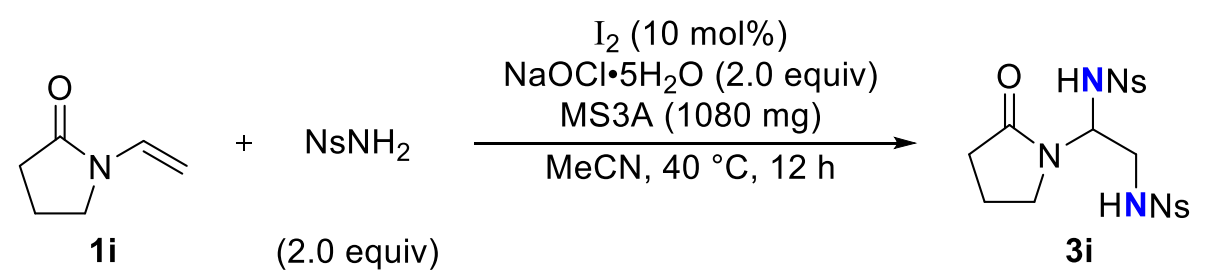

Following the general procedure (conditions I), 1-ethenyl-2-pyrrolidinone (1i: $55.9 \mathrm{mg}, 0.49$ $\mathrm{mmol}$ ), iodine (13.4 mg, $0.05 \mathrm{mmol})$, 2-nitrobenzenesulfonamide (203.5 mg, $1.01 \mathrm{mmol}$ ), sodium hypochlorite pentahydrate $(163.8 \mathrm{mg}, 1.00 \mathrm{mmol})$ and MS3A (1086.5 mg) were stirred at $40{ }^{\circ} \mathrm{C}$ in $\mathrm{MeCN}$ for $12 \mathrm{~h}$. Washing the crude product with $\mathrm{Et}_{2} \mathrm{O}$ gave the product $(3 \mathrm{i}: 170.1$ mg, 66\%) as a white solid. mp: $159.7-160.1{ }^{\circ} \mathrm{C}$; TLC: $\mathrm{R}_{\mathrm{f}}=0.25$ (EtOAc); ${ }^{1} \mathrm{H}$ NMR $(400 \mathrm{MHz}$, $\left.\mathrm{CDCl}_{3}\right) \delta 8.79(\mathrm{~d}, J=8.8 \mathrm{~Hz}, 1 \mathrm{H}), 8.40(\mathrm{dd}, J=5.6,5.6 \mathrm{~Hz}, 1 \mathrm{H}), 8.10-7.75(\mathrm{~m}, 8 \mathrm{H}), 5.52-$ $5.41(\mathrm{~m}, 1 \mathrm{H}), 3.32-3.25(\mathrm{~m}, 1 \mathrm{H}), 3.21-3.09(\mathrm{~m}, 2 \mathrm{H}), 3.08-2.98(\mathrm{~m}, 1 \mathrm{H}), 2.07-1.91(\mathrm{~m}, 1 \mathrm{H})$, 
$1.71-1.55(\mathrm{~m}, 2 \mathrm{H}), 1.19-1.03(\mathrm{~m}, 1 \mathrm{H}) ;{ }^{13} \mathrm{C} \mathrm{NMR}\left(100 \mathrm{MHz}, \mathrm{DMSO}-d_{6}\right) \delta 174.0,147.5,147.2$, $134.5,134.2$, 132.9, 132.8, 132.5, 132.4, 130.2, 129.3, 124.64, 124.58, 59.9, 43.0, 40.6, 30.3, 17.2; IR (ATR) 3337, 1369, 1350, 1167, 1078, 1069, 853, 781, $743 \mathrm{~cm}^{-1}$; HRMS (FAB+): $\mathrm{m} / z$ calcd. for $\mathrm{C}_{18} \mathrm{H}_{20} \mathrm{~N}_{5} \mathrm{O}_{9} \mathrm{~S}_{2}\left([\mathrm{M}+\mathrm{H}]^{+}\right)$514.0702, found 514.0695.

\section{$N, N^{\prime}$-(3-((4-methylphenyl)sulfonamido)propane-1,2-diyl)bis(2-}

\section{nitrobenzenesulfonamide) (3j)}

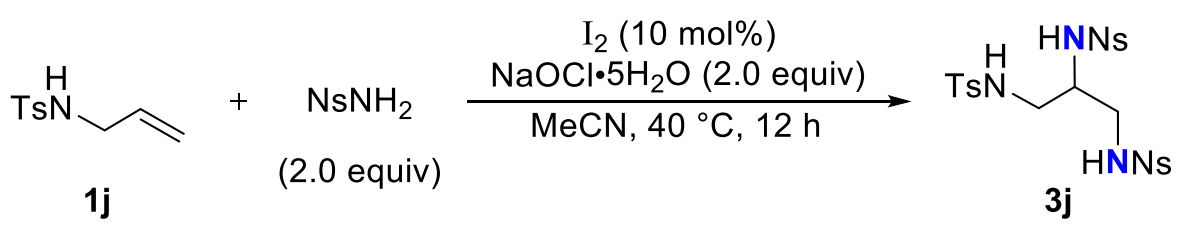

Following the general procedure (conditions I), $N$-allyl-4-methylbenzenesulfonamide (1j: $104.7 \mathrm{mg}, 0.50 \mathrm{mmol}$ ), iodine (13.0 mg, $0.05 \mathrm{mmol}), 2$-nitrobenzenesulfonamide (202.4 mg, $1.00 \mathrm{mmol})$ and sodium hypochlorite pentahydrate $(164.5 \mathrm{mg}, 1.00 \mathrm{mmol})$ were stirred at $40^{\circ} \mathrm{C}$ in $\mathrm{MeCN}$ for $12 \mathrm{~h}$. Purification by flash column chromatography on silica gel (hexane/EtOAc $=1: 1)$ gave the product $(\mathbf{3 j}: 144.7 \mathrm{mg}, 48 \%)$ as a white solid. $\mathrm{mp}: 88.6-89.2{ }^{\circ} \mathrm{C}$; TLC: $\mathrm{R}_{\mathrm{f}}=$ 0.28 (hexane/EtOAc $=1: 1) ;{ }^{1} \mathrm{H}$ NMR $\left(400 \mathrm{MHz}, \mathrm{CDCl}_{3}\right) \delta 8.19-8.11(\mathrm{~m}, 1 \mathrm{H}), 8.07-7.97$ (m, 1H), 7.95-7.68 (m, 6H), $7.64(\mathrm{~d}, J=8.0 \mathrm{~Hz}, 2 \mathrm{H}), 7.26(\mathrm{~d}, J=8.0 \mathrm{~Hz}, 2 \mathrm{H}), 6.11(\mathrm{~d}, J=7.6 \mathrm{~Hz}$, $1 \mathrm{H}), 5.94(\mathrm{dd}, J=6.4,6.4 \mathrm{~Hz}, 1 \mathrm{H}), 5.34$ (t, $J=6.8 \mathrm{~Hz}, 1 \mathrm{H}), 3.85-3.71(\mathrm{~m}, 1 \mathrm{H}), 3.40-3.21(\mathrm{~m}$, 2H), 3.21-3.00 (m, 2H), 2.40 (s, 3H); $\left.{ }^{13} \mathrm{C} \mathrm{NMR} \mathrm{(100} \mathrm{MHz,} \mathrm{CDCl}_{3}\right) \delta$ 147.7, 147.6, 143.9, 135.9, 133.9, 133.4, 133.2, 133.1, 132.8, 131.0, 130.9, 129.9, 127.0, 125.63 125.57, 54.2, 44.2, 43.9, 21.5 (One $\mathrm{sp}^{2}$ signal was not observed because of overlapping.); IR (ATR) 3324, 1535, $1418,1337,1159,1122,1089,982,854,815,782,739 \mathrm{~cm}^{-1}$; HRMS (FAB+): $m / z$ calcd. for $\mathrm{C}_{22} \mathrm{H}_{24} \mathrm{~N}_{5} \mathrm{O}_{10} \mathrm{~S}_{3}\left([\mathrm{M}+\mathrm{H}]^{+}\right)$614.0685, found 614.0688.

\section{$N, N^{\prime}$-(1-phenylethane-1,2-diyl)bis(2-nitrobenzenesulfonamide) (3k)}

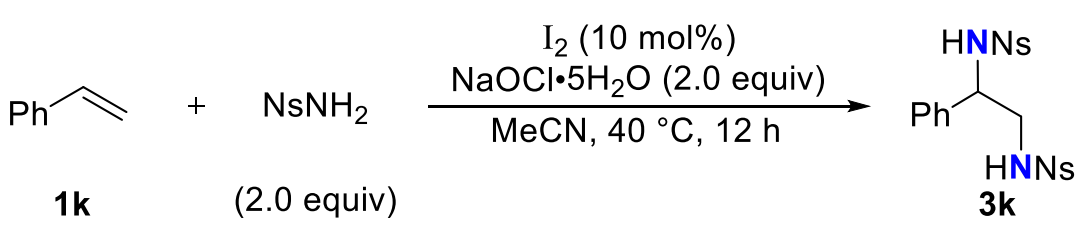

(0.5 mmol scale)

Following the general procedure (conditions I), styrene (1k: $52.0 \mathrm{mg}, 0.50 \mathrm{mmol})$, iodine (13.0 $\mathrm{mg}, 0.05 \mathrm{mmol}), 2$-nitrobenzenesulfonamide $(202.4 \mathrm{mg}, 1.00 \mathrm{mmol})$ and sodium hypochlorite pentahydrate $(165.5 \mathrm{mg}, 1.01 \mathrm{mmol})$ were stirred at $40{ }^{\circ} \mathrm{C}$ in $\mathrm{MeCN}$ for $12 \mathrm{~h}$. Washing the 
crude product with $\mathrm{Et}_{2} \mathrm{O}$ gave the product (3k: $194.4 \mathrm{mg}, 77 \%$ ) as a white solid. $\mathrm{mp}: 175.3-$ $176.4{ }^{\circ} \mathrm{C}$; TLC: $\mathrm{R}_{\mathrm{f}}=0.30$ (hexane/EtOAc $\left.=1: 1\right) ;{ }^{1} \mathrm{H}$ NMR $\left(400 \mathrm{MHz}, \mathrm{DMSO}-d_{6}\right) \delta 8.64(\mathrm{~d}, J$ $=9.6 \mathrm{~Hz}, 1 \mathrm{H}), 8.16(\mathrm{dd}, J=5.6,5.6 \mathrm{~Hz}, 1 \mathrm{H}), 7.99-7.91(\mathrm{~m}, 1 \mathrm{H}), 7.89-7.72(\mathrm{~m}, 4 \mathrm{H}), 7.66(\mathrm{dd}$, $J=7.2,7.2 \mathrm{~Hz}, 2 \mathrm{H}), 7.52(\mathrm{dd}, J=7.2,7.2 \mathrm{~Hz}, 1 \mathrm{H}), 7.20-7.01(\mathrm{~m}, 5 \mathrm{H}), 4.52(\mathrm{dd}, J=6.8,15.6$ $\mathrm{Hz}, 1 \mathrm{H}), 3.30-3.11(\mathrm{~m}, 2 \mathrm{H}) ;{ }^{13} \mathrm{C}$ NMR (100 MHz, DMSO- $\left.d_{6}\right) \delta 147.5,147.0,138.0,134.1$, 133.8, 132.9, 132.7, 132.2, 129.9, 129.4, 128.1, 127.6, 127.1, 124.6, 124.1, 57.8, 47.8 (One sp ${ }^{2}$ signal was not observed because of overlapping.); IR (ATR) 3854, 3337, 3079, 1527, 1446, 1341, 1167, 1121, 869, $785 \mathrm{~cm}^{-1}$; HRMS (FAB+): $\mathrm{m} / z$ calcd. for $\mathrm{C}_{20} \mathrm{H}_{19} \mathrm{~N}_{4} \mathrm{O}_{8} \mathrm{~S}_{2}\left([\mathrm{M}+\mathrm{H}]^{+}\right)$ 507.0644 , found 507.0649.

(gram scale)

Following the general procedure (conditions I), styrene (1k: $527.7 \mathrm{mg}, 5.1 \mathrm{mmol}$ ), iodine (126.6 mg, $0.50 \mathrm{mmol}$ ), 2-nitrobenzenesulfonamide (2024.0 mg, $10.0 \mathrm{mmol}$ ) and sodium hypochlorite pentahydrate $(1645.5 \mathrm{mg}, 10.0 \mathrm{mmol})$ were stirred at $40{ }^{\circ} \mathrm{C}$ in $\mathrm{MeCN}$ for $12 \mathrm{~h}$. Washing the crude product with $\mathrm{Et}_{2} \mathrm{O}$ gave the product (3k: $1811.9 \mathrm{mg}, 71 \%$ ) as a white solid.

\section{$N, N^{\prime}$-(1-(4-fluorophenyl)ethane-1,2-diyl)bis(2-nitrobenzenesulfonamide) (3I)}

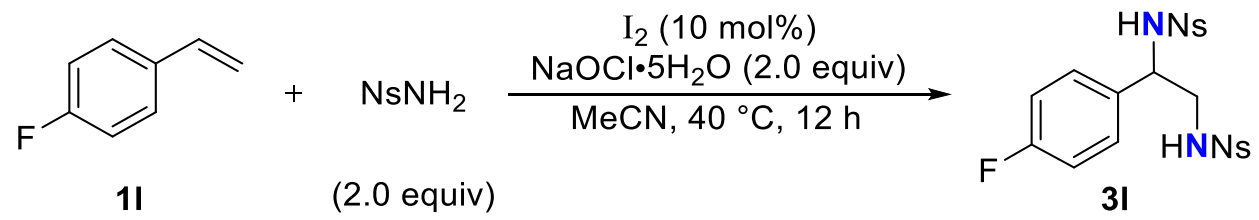

Following the general procedure (condition I), 4-fluorostyrene (11: $61.8 \mathrm{mg}, 0.51 \mathrm{mmol}$ ), iodine (12.6 mg, $0.05 \mathrm{mmol}$ ), 2-nitrobenzenesulfonamide (203.4 mg, $1.01 \mathrm{mmol}$ ) and sodium hypochlorite pentahydrate $(167.8 \mathrm{mg}, 1.02 \mathrm{mmol})$ were stirred at $40{ }^{\circ} \mathrm{C}$ in $\mathrm{MeCN}$ for $12 \mathrm{~h}$. Washing the crude product with $\mathrm{Et}_{2} \mathrm{O}$ gave the product (31: $184.4 \mathrm{mg}, 69 \%$ ) as a white solid. mp: 192.2-192.7 ${ }^{\circ} \mathrm{C}$; TLC: $\mathrm{R}_{\mathrm{f}}=0.28$ (hexane/EtOAc $\left.=1: 1\right) ;{ }^{1} \mathrm{H}$ NMR (400 MHz, DMSO- $\left.d_{6}\right)$ $\delta 8.67(\mathrm{~d}, J=9.2 \mathrm{~Hz}, 1 \mathrm{H}), 8.17(\mathrm{dd}, J=5.6,5.6 \mathrm{~Hz}, 1 \mathrm{H}), 7.93(\mathrm{~d}, J=8.0 \mathrm{~Hz}, 1 \mathrm{H}), 7.89-7.76$ (m, 4H), 7.75-7.65 (dd, $J=7.2,7.2 \mathrm{~Hz}, 2 \mathrm{H}), 7.57(\mathrm{dd}, J=7.2,7.2 \mathrm{~Hz}, 1 \mathrm{H}), 7.21-7.10$ (dd, $J$ $=6.0,8.8 \mathrm{~Hz}, 2 \mathrm{H}), 6.88(\mathrm{dd}, J=8.8,8.8 \mathrm{~Hz}, 2 \mathrm{H}), 4.54(\mathrm{dd}, J=9.2,15.6 \mathrm{~Hz}, 1 \mathrm{H}), 3.29-3.15$ $(\mathrm{m}, 2 \mathrm{H}) ;{ }^{13} \mathrm{C}$ NMR $\left(100 \mathrm{MHz}, \mathrm{DMSO}-d_{6}\right) \delta 161.4\left(\mathrm{~d}, J_{\mathrm{C}-\mathrm{F}}=242.9 \mathrm{~Hz}\right), 147.4,147.0,134.3$, $134.2,133.9\left(\mathrm{~d}, J_{\mathrm{C}-\mathrm{F}}=13.2 \mathrm{~Hz}\right), 132.8,132.72,132.69,132.2,129.9,129.4,129.2\left(\mathrm{~d}, J_{\mathrm{C}-\mathrm{F}}=\right.$ $8.3 \mathrm{~Hz}), 124.5,124.1,114.7\left(\mathrm{~d}, J_{\mathrm{C}-\mathrm{F}}=20.5 \mathrm{~Hz}\right), 56.9,47.8 ;{ }^{19} \mathrm{~F}$ NMR $\left(377 \mathrm{MHz}, \mathrm{DMSO}-d_{6}\right) \delta$ -114.0; IR (ATR) 3331, 3100, 1528, 1412, 1344, 1229, 1167, 1098, 949, 854, $743 \mathrm{~cm}^{-1}$; HRMS (FAB-): $m / z$ calcd. for $\mathrm{C}_{20} \mathrm{H}_{16} \mathrm{FN}_{4} \mathrm{O}_{8} \mathrm{~S}_{2}\left([\mathrm{M}-\mathrm{H}]^{-}\right)$523.0394, found 523.0401 . 


\section{$N, N^{\prime}$-(1-(4-chlorophenyl)ethane-1,2-diyl)bis(2-nitrobenzenesulfonamide) (3m)}

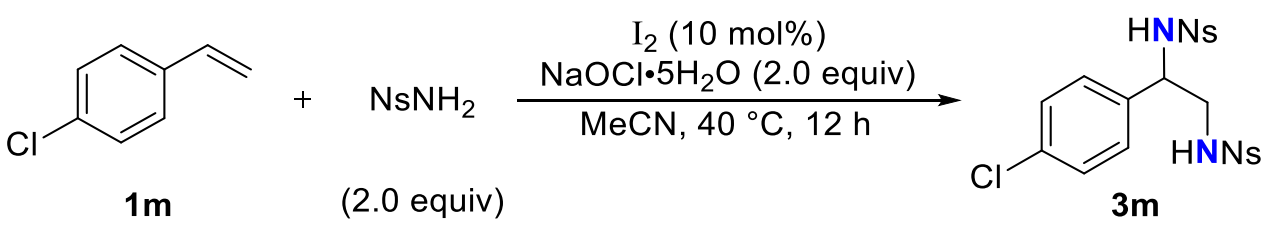

Following the general procedure (conditions I), 4-chlorostyrene (1m: $65.7 \mathrm{mg}, 0.47 \mathrm{mmol}$ ), iodine (13.2 mg, $0.05 \mathrm{mmol}), 2$-nitrobenzenesulfonamide (202.3 mg, $1.00 \mathrm{mmol}$ ) and sodium hypochlorite pentahydrate $(167.5 \mathrm{mg}, 1.02 \mathrm{mmol})$ were stirred at $40{ }^{\circ} \mathrm{C}$ in $\mathrm{MeCN}$ for $12 \mathrm{~h}$. Washing the crude product with $\mathrm{Et}_{2} \mathrm{O}$ gave the product (3m: $199.3 \mathrm{mg}, 78 \%$ ) as a white solid. mp: 211.2-211.9 ${ }^{\circ} \mathrm{C}$; TLC: $\mathrm{R}_{\mathrm{f}}=0.31$ (hexane/EtOAc $\left.=1: 1\right) ;{ }^{1} \mathrm{H}$ NMR (400 MHz, DMSO- $\left.d_{6}\right)$ $\delta 8.38$ (brs, $1 \mathrm{H}), 7.92(\mathrm{~d}, J=8.0 \mathrm{~Hz}, 1 \mathrm{H}), 7.88-7.75(\mathrm{~m}, 4 \mathrm{H}), 7.70(\mathrm{dd}, J=8.0,8.0 \mathrm{~Hz}, 2 \mathrm{H})$, $7.57(\mathrm{dd}, J=8.0,8.0 \mathrm{~Hz}, 1 \mathrm{H}), 7.16(\mathrm{~d}, J=8.8 \mathrm{~Hz}, 2 \mathrm{H}), 7.11(\mathrm{~d}, J=8.8 \mathrm{~Hz}, 2 \mathrm{H}), 4.51(\mathrm{dd}, J=$ 7.1, $7.1 \mathrm{~Hz}, 1 \mathrm{H}), 3.23$ (d, $J=7.1 \mathrm{~Hz}, 2 \mathrm{H})\left(\mathrm{One} \mathrm{N}-\mathrm{H}\right.$ signal was not observed.); ${ }^{13} \mathrm{C}$ NMR (100 MHz, DMSO-d6) $\delta 147.3,147.0,137.1,133.9,133.8,132.8,132.72,132.66,132.22,132.18$, 129.9, 129.4, 129.0, 127.9, 124.5, 124.1, 56.9, 47.7; IR (ATR) 3383, 3329, 3013, 3021, 2884, 1526, 1406, 1342, 1167, 1090, 1013, 854, 743, $729 \mathrm{~cm}^{-1}$; HRMS (FAB-): $\mathrm{m} / z$ calcd. for $\mathrm{C}_{20} \mathrm{H}_{16} \mathrm{ClN}_{4} \mathrm{O}_{8} \mathrm{~S}_{2}\left([\mathrm{M}-\mathrm{H}]^{-}\right)$539.0098, found 539.0096.

\section{$N, N^{\prime}$-(1-(4-bromophenyl)ethane-1,2-diyl)bis(2-nitrobenzenesulfonamide) (3n)}

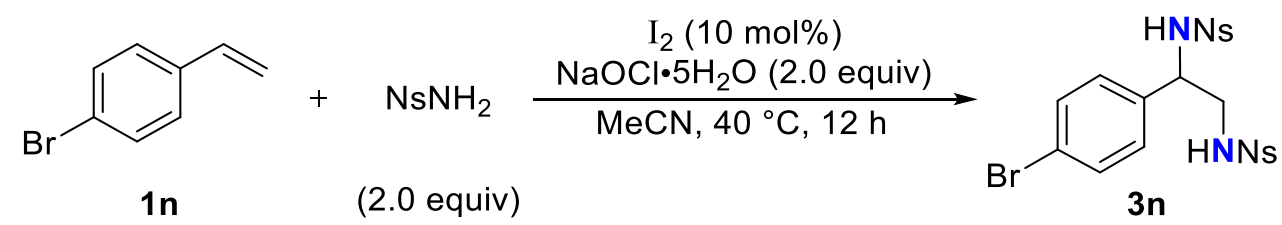

Following the general procedure (conditions I), 4-bromostyrene (1n: $92.4 \mathrm{mg}, 0.50 \mathrm{mmol}$ ), iodine (12.6 mg, $0.05 \mathrm{mmol}$ ), 2-nitrobenzenesulfonamide (203.2 mg, $1.01 \mathrm{mmol}$ ) and sodium hypochlorite pentahydrate $(164.4 \mathrm{mg}, 1.00 \mathrm{mmol})$ were stirred at $40{ }^{\circ} \mathrm{C}$ in $\mathrm{MeCN}$ for $12 \mathrm{~h}$. Washing the crude product with $\mathrm{Et}_{2} \mathrm{O}$ gave the product (3n: $234.1 \mathrm{mg}, 79 \%$ ) as a white solid. mp: $214.0-215.1{ }^{\circ} \mathrm{C}$; TLC: $\mathrm{R}_{\mathrm{f}}=0.33$ (hexane/EtOAc $\left.=1: 1\right) ;{ }^{1} \mathrm{H}$ NMR (400 MHz, DMSO- $\left.d_{6}\right)$ $\delta 8.71(\mathrm{~d}, J=9.2 \mathrm{~Hz}, 1 \mathrm{H}), 8.16(\mathrm{dd}, J=6.0,6.0 \mathrm{~Hz}, 1 \mathrm{H}), 7.92(\mathrm{~d}, J=7.2 \mathrm{~Hz}, 1 \mathrm{H}), 7.89-7.62$ $(\mathrm{m}, 6 \mathrm{H}), 7.58(\mathrm{dd}, J=7.2,7.2 \mathrm{~Hz}, 1 \mathrm{H}), 7.24(\mathrm{~d}, J=8.0 \mathrm{~Hz}, 2 \mathrm{H}), 7.16(\mathrm{~d}, J=8.0 \mathrm{~Hz}, 2 \mathrm{H}), 4.51$ $(\mathrm{dd}, J=7.6,16.4 \mathrm{~Hz}, 1 \mathrm{H}), 3.31-3.15(\mathrm{~m}, 2 \mathrm{H}) ;{ }^{13} \mathrm{C} \mathrm{NMR}\left(100 \mathrm{MHz}, \mathrm{DMSO}-d_{6}\right) \delta 147.3,147.0$, $137.5,133.9,133.8,132.8,132.6,132.3,130.8,129.9,129.3,124.5,124.2,120.9,56.9,47.6$ (Two $\mathrm{sp}^{2}$ signals were not observed because of overlapping.); IR (ATR) 3335, 3103, 1530, 
1423, 1346, 1165, 1096, 1076, 951, 854, $779 \mathrm{~cm}^{-1}$; HRMS (FAB-): $m / z$ calcd. for $\mathrm{C}_{20} \mathrm{H}_{16} \mathrm{BrN}_{4} \mathrm{O}_{8} \mathrm{~S}_{2}\left([\mathrm{M}-\mathrm{H}]^{-}\right)$582.9593, found 582.9588.

\section{$N, N^{\prime}$-(1-(4-acetoxyphenyl)ethane-1,2-diyl)bis(2-nitrobenzenesulfonamide) (3o)}

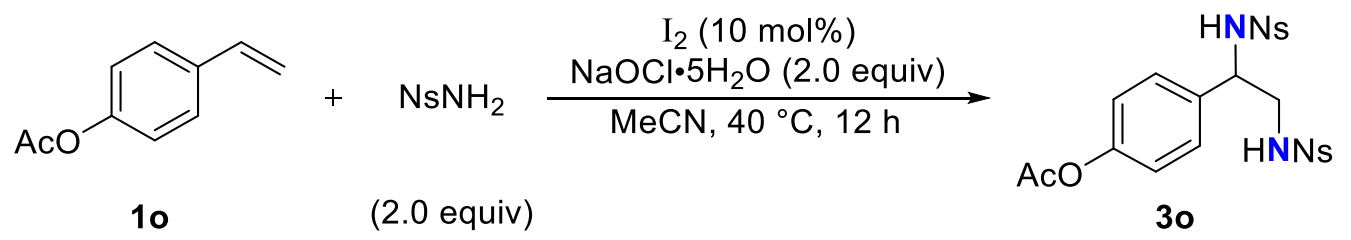

Following the general procedure (conditions I), 4-acetoxystyrene (1o: $78.0 \mathrm{mg}, 0.48 \mathrm{mmol}$ ), iodine (13.0 mg, $0.05 \mathrm{mmol})$, 2-nitrobenzenesulfonamide (202.0 mg, $1.00 \mathrm{mmol}$ ) and sodium hypochlorite pentahydrate $(165.5 \mathrm{mg}, 1.01 \mathrm{mmol})$ were stirred at $40{ }^{\circ} \mathrm{C}$ in $\mathrm{MeCN}$ for $12 \mathrm{~h}$. Washing the crude product with $\mathrm{Et}_{2} \mathrm{O}$ gave the product (3o: $207.4 \mathrm{mg}, 76 \%$ ) as a white solid. mp: $150.7-151.5^{\circ} \mathrm{C}$; TLC: $\mathrm{R}_{\mathrm{f}}=0.33$ (hexane/EtOAc $\left.=1: 1\right) ;{ }^{1} \mathrm{H}$ NMR (400 MHz, DMSO- $d_{6}$ ) $\delta 8.67$ (brs, 1H), 8.18 (brs, 1H), $7.94(\mathrm{~d}, J=7.8 \mathrm{~Hz}, 1 \mathrm{H}), 7.90-7.78(\mathrm{~m}, \quad 4 \mathrm{H}), 7.68$ (dd, $J=$ 7.3, 7.3 Hz, 2H), $7.56(\mathrm{dd}, J=7.3,7.3 \mathrm{~Hz}, 1 \mathrm{H}), 7.18$ (d, $J=8.3 \mathrm{~Hz}, 2 \mathrm{H}), 6.86(\mathrm{~d}, J=8.3 \mathrm{~Hz}$, 2H), 4.60-4.49 (m, 1H), 3.29-3.12 (m, 2H), $2.22(\mathrm{~s}, 3 \mathrm{H}) ;{ }^{13} \mathrm{C}$ NMR (100 MHz, DMSO- $\left.d_{6}\right) \delta$ 168.9, 149.7, 147.4, 147.0, 135.6, 134.1, 133.9, 132.8, 132.7, 132.5, 132.2, 129.8, 129.3, 128.2, 124.5, 124.1, 121.3, 57.0, 47.5, 20.8; IR (ATR) 3343, 3092, 2941, 1748, 1539, 1431, 1360, 1337, 1204, 1157, 1111, 1086, 1016, $856 \mathrm{~cm}^{-1}$; HRMS (FAB+): $m / z$ calcd. for $\mathrm{C}_{22} \mathrm{H}_{21} \mathrm{~N}_{4} \mathrm{O}_{10} \mathrm{~S}_{2}$ $\left([\mathrm{M}+\mathrm{H}]^{+}\right)$565.0699, found 565.0706.

\section{$N, N^{\prime}$-(1-(3-methoxyphenyl)ethane-1,2-diyl)bis(2-nitrobenzenesulfonamide) (3p)}
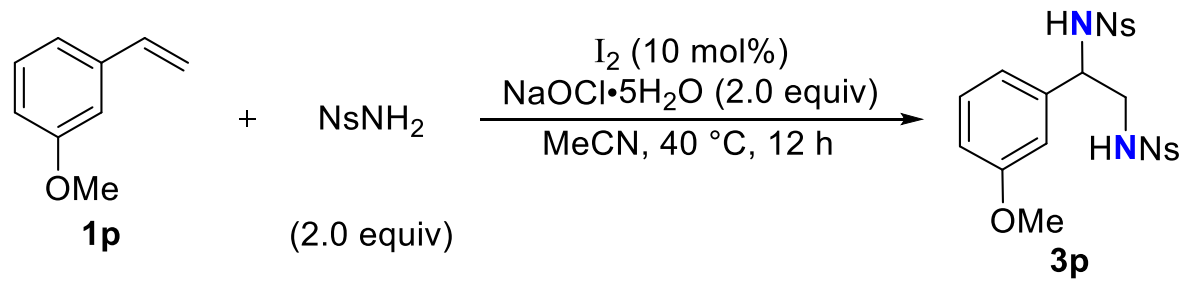

Following the general procedure (conditions I), 3-vinylanisole (1p: $68.0 \mathrm{mg}, 0.51 \mathrm{mmol}$ ), iodine (12.7 mg, $0.05 \mathrm{mmol}$ ), 2-nitrobenzenesulfonamide (202.7 mg, $1.00 \mathrm{mmol}$ ) and sodium hypochlorite pentahydrate $(164.8 \mathrm{mg}, 1.00 \mathrm{mmol})$ were stirred at $40{ }^{\circ} \mathrm{C}$ in $\mathrm{MeCN}$ for $12 \mathrm{~h}$. Washing the crude product with $\mathrm{Et}_{2} \mathrm{O}$ gave the product (3p: $191.2 \mathrm{mg}, 70 \%$ ) as a white solid. mp: 165.2-166.0 ${ }^{\circ} \mathrm{C}$; TLC: $\mathrm{R}_{\mathrm{f}}=0.40$ (hexane/EtOAc $\left.=1: 1\right) ;{ }^{1} \mathrm{H}$ NMR (400 MHz, DMSO- $\left.d_{6}\right)$ $\delta 8.62(\mathrm{~d}, J=8.8 \mathrm{~Hz}, 1 \mathrm{H}), 8.18(\mathrm{dd}, J=5.6,5.6 \mathrm{~Hz}, 1 \mathrm{H}), 7.93(\mathrm{~d}, J=7.6 \mathrm{~Hz}, 1 \mathrm{H}), 7.91-7.78$ (m, 4H), 7.72-7.61 (m, 2H), $7.52(\mathrm{dd}, J=7.6,7.6 \mathrm{~Hz}, 1 \mathrm{H}), 6.98(\mathrm{dd}, J=8.0,8.0 \mathrm{~Hz}, 1 \mathrm{H})$, 
6.79-6.68 (m, 2H), 6.63-6.55 (m, 1H), $4.51(\mathrm{dd}, J=7.2,16.0 \mathrm{~Hz}, 1 \mathrm{H}), 3.60(\mathrm{~s}, 3 \mathrm{H}), 3.31-3.15$ $(\mathrm{m}, 2 \mathrm{H}) ;{ }^{13} \mathrm{C}$ NMR $\left(100 \mathrm{MHz}, \mathrm{DMSO}-d_{6}\right) \delta 158.9,147.4,147.0,139.4,134.0,133.7,132.79$, $132.77,132.69$, 132.1 129.9, 129.4, 129.0, 124.5, 123.9, 119.4, 113.3, 112.3, 57.7, 54.8, 47.8; IR (ATR) 3302, 3096, 1589, 1531, 1491, 1435, 1358, 1337, 1263, 1155, 1121, 1051, 854, 779, $729 \mathrm{~cm}^{-1}$; HRMS (FAB+): $\mathrm{m} / z$ calcd. for $\mathrm{C}_{21} \mathrm{H}_{21} \mathrm{~N}_{4} \mathrm{O}_{9} \mathrm{~S}_{2}\left([\mathrm{M}+\mathrm{H}]^{+}\right)$537.0750, found 537.0756.

\section{$N, N^{\prime}$-(1-(2-methylphenyl)ethane-1,2-diyl)bis(2-nitrobenzenesulfonamide) (3q)}

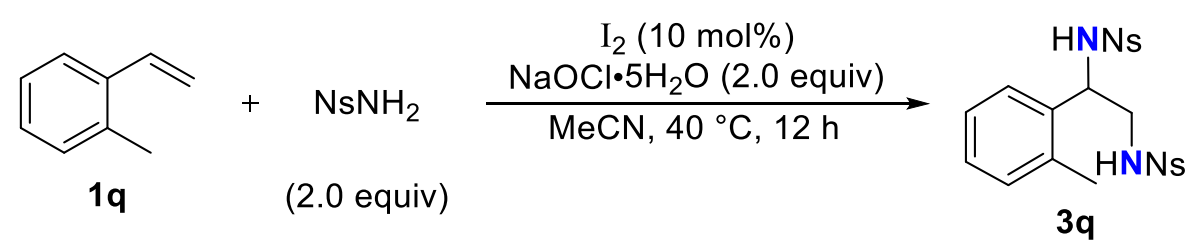

Following the general procedure (conditions I), 2-methylstyrene (1q: $56.5 \mathrm{mg}, 0.48 \mathrm{mmol}$ ), iodine (13.0 mg, $0.05 \mathrm{mmol})$, 2-nitrobenzenesulfonamide (202.0 mg, $1.00 \mathrm{mmol}$ ) and sodium hypochlorite pentahydrate $(164.5 \mathrm{mg}, 1.00 \mathrm{mmol})$ were stirred at $40{ }^{\circ} \mathrm{C}$ in $\mathrm{MeCN}$ for $12 \mathrm{~h}$. Purification by flash column chromatography on silica gel $\left(\mathrm{CH}_{2} \mathrm{Cl}_{2} / \mathrm{MeOH}=19: 1\right)$ and washing with $\mathrm{Et}_{2} \mathrm{O}$ gave the product (3q: $149.6 \mathrm{mg}, 59 \%$ ) as a white solid. mp: $219.4-220.0{ }^{\circ} \mathrm{C}$; TLC: $\mathrm{R}_{\mathrm{f}}=0.58$ (hexane/EtOAc $\left.=1: 1\right) ;{ }^{1} \mathrm{H}$ NMR (400 MHz, DMSO-d 6 ) $\delta 8.62$ (brs, $\left.1 \mathrm{H}\right), 8.28$ (brs, 1H), 8.00-7.85 (m, 2H), 7.85-7.71 (m, 3H), 7.69-7.52 (m, 2H), 7.50-7.41 (m, 1H), 7.29$7.21(\mathrm{~m}, 1 \mathrm{H}), 7.00-6.82(\mathrm{~m}, 3 \mathrm{H}), 4.82-4.71(\mathrm{~m}, 1 \mathrm{H}), 3.29-3.05(\mathrm{~m}, 2 \mathrm{H}), 2.20(\mathrm{~s}, 3 \mathrm{H})$; ${ }^{13} \mathrm{C}$ NMR (100 MHz, DMSO-d6) $\delta 147.5,146.9,136.4,135.1,134.0,133.6,132.92,132.86,132.8$, 132.1, 129.9, 129.5, 129.3, 127.4, 126.3, 125.9, 124.5, 124.0, 54.1, 47.1, 18.6; IR (ATR) 3339, 3094, 1593, 1530, 1416, 1346, 1304, 1169, 1061, 949, 854, $754 \mathrm{~cm}^{-1}$; HRMS (FAB+): $\mathrm{m} / z$ calcd. for $\mathrm{C}_{21} \mathrm{H}_{21} \mathrm{~N}_{4} \mathrm{O}_{8} \mathrm{~S}_{2}\left([\mathrm{M}+\mathrm{H}]^{+}\right)$521.0801, found 521.0805 .

\section{$N, N^{\prime}$-(1-(3-methylphenyl)ethane-1,2-diyl)bis(2-nitrobenzenesulfonamide) (3r)}

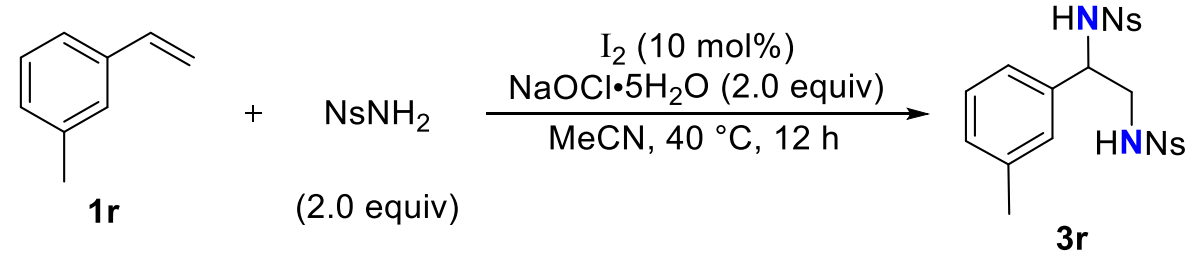

Following the general procedure (conditions I), 3-methylstyrene (1r: $57.6 \mathrm{mg}, 0.49 \mathrm{mmol}$ ), iodine (13.2 mg, $0.05 \mathrm{mmol})$, 2-nitrobenzenesulfonamide (202.8 mg, $1.00 \mathrm{mmol}$ ) and sodium hypochlorite pentahydrate $(166.0 \mathrm{mg}, 1.01 \mathrm{mmol})$ were stirred at $40{ }^{\circ} \mathrm{C}$ in $\mathrm{MeCN}$ for $12 \mathrm{~h}$. Washing the crude product with $\mathrm{Et}_{2} \mathrm{O}$ gave the product (3r: $203.2 \mathrm{mg}, 80 \%$ ) as a white solid. mp: 216.1-217.2 ${ }^{\circ} \mathrm{C}$; TLC: $\mathrm{R}_{\mathrm{f}}=0.58$ (hexane/EtOAc $\left.=1: 1\right) ;{ }^{1} \mathrm{H}$ NMR $\left(400 \mathrm{MHz}\right.$, DMSO- $\left.d_{6}\right)$ 
$\delta 8.25$ (brs, 1H), $7.95(\mathrm{~d}, J=7.6 \mathrm{~Hz}, 1 \mathrm{H}), 7.89-7.74(\mathrm{~m}, 4 \mathrm{H}), 7.66(\mathrm{dd}, J=7.6,7.6 \mathrm{~Hz}, 2 \mathrm{H})$, $7.52(\mathrm{dd}, J=7.6,7.6 \mathrm{~Hz}, 1 \mathrm{H}), 7.00-6.79(\mathrm{~m}, 4 \mathrm{H}), 4.48(\mathrm{dd}, J=6.8,6.8 \mathrm{~Hz}, 1 \mathrm{H}), 3.32-3.17$ (m, $2 \mathrm{H}), 2.08(\mathrm{~s}, 3 \mathrm{H})\left(\right.$ One N-H signal was not observed because of overlapping.); ${ }^{13} \mathrm{C}$ NMR (100 MHz, DMSO-d $d_{6} \delta 147.4,147.0,137.7,137.0,134.0,133.7,132.83,132.78,132.7,132.0$, 129.8, 129.4, 128.2, 127.9, 127.7, 124.5, 124.1, 123.9, 57.7, 47.7, 20.8; IR (ATR) 3312, 1539, 1528, 1445, 1354, 1337, 1323, 1157, 1123, 1067, 854, $781 \mathrm{~cm}^{-1}$; HRMS (FAB+): $m / z$ calcd. for $\mathrm{C}_{21} \mathrm{H}_{21} \mathrm{~N}_{4} \mathrm{O}_{8} \mathrm{~S}_{2}\left([\mathrm{M}+\mathrm{H}]^{+}\right)$521.0801, found 521.0797.

\section{$N, N^{\prime}$-(1-(4-methylphenyl)ethane-1,2-diyl)bis(2-nitrobenzenesulfonamide) (3s)}
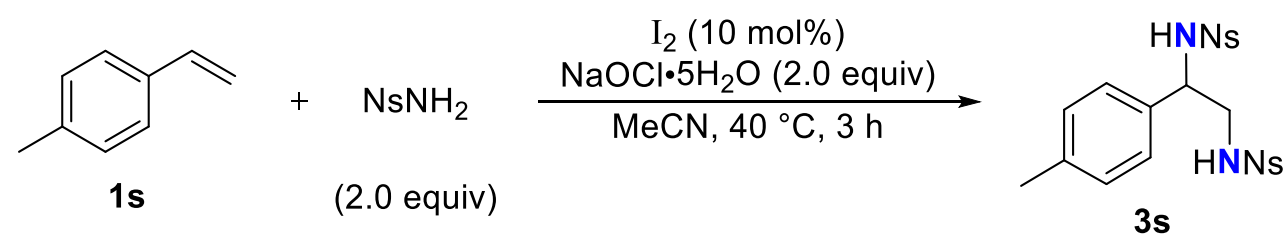

Following the general procedure (conditions I), 4-methylstyrene (1s: $59.4 \mathrm{mg}, 0.50 \mathrm{mmol}$ ), iodine (12.9 mg, $0.05 \mathrm{mmol})$, 2-nitrobenzenesulfonamide (203.2 mg, $1.01 \mathrm{mmol}$ ) and sodium hypochlorite pentahydrate $(166.5 \mathrm{mg}, 1.01 \mathrm{mmol})$ were stirred at $40{ }^{\circ} \mathrm{C}$ in $\mathrm{MeCN}$ for $3 \mathrm{~h}$. Washing the crude product with $\mathrm{Et}_{2} \mathrm{O}$ gave the product (3s: $\left.184.3 \mathrm{mg}, 70 \%\right)$ as a white solid. mp: 201.2-201.9 ${ }^{\circ} \mathrm{C}$; TLC: $\mathrm{R}_{\mathrm{f}}=0.38$ (hexane/EtOAc $\left.=1: 1\right) ;{ }^{1} \mathrm{H}$ NMR (400 MHz, DMSO- $\left.d_{6}\right)$ $\delta 8.55(\mathrm{~d}, J=8.8 \mathrm{~Hz}, 1 \mathrm{H}), 8.09(\mathrm{dd}, J=6.0,6.0 \mathrm{~Hz}, 1 \mathrm{H}), 7.92(\mathrm{~d}, J=8.0 \mathrm{~Hz}, 1 \mathrm{H}), 7.88-7.73$ (m, 4H), 7.71-7.61 (m, 2H), $7.53(\mathrm{dd}, J=8.0,8.0 \mathrm{~Hz}, 1 \mathrm{H}), 7.01(\mathrm{~d}, J=8.0 \mathrm{~Hz}, 2 \mathrm{H}), 6.85(\mathrm{~d}, J$ $=8.0 \mathrm{~Hz}, 2 \mathrm{H}), 4.46(\mathrm{dd}, J=7.2,15.6 \mathrm{~Hz}, 1 \mathrm{H}), 3.29-3.14(\mathrm{~m}, 2 \mathrm{H}), 2.14(\mathrm{~s}, 3 \mathrm{H}) ;{ }^{13} \mathrm{C} \mathrm{NMR}(100$ MHz, DMSO- $\left.d_{6}\right) \delta 147.4,147.0,136.7,135.1,133.9,133.6,132.9,132.8,132.7,132.1,129.8$, 129.4, 128.5, 127.0, 124.5, 124.0, 57.4, 47.8, 20.6; IR (ATR) 3333, 3102, 1531, 1440, 1366, $1337,1159,1125,854,818,783,731 \mathrm{~cm}^{-1}$; HRMS (FAB-): $m / z$ calcd. for $\mathrm{C}_{21} \mathrm{H}_{19} \mathrm{~N}_{4} \mathrm{O}_{8} \mathrm{~S}_{2}([\mathrm{M}-$ $\left.\mathrm{H}]^{-}\right)$519.0644, found 519.0653 .

\section{$N, N^{\prime}$-(2-phenylpropane-1,2-diyl)bis(2-nitrobenzenesulfonamide) (3t)}

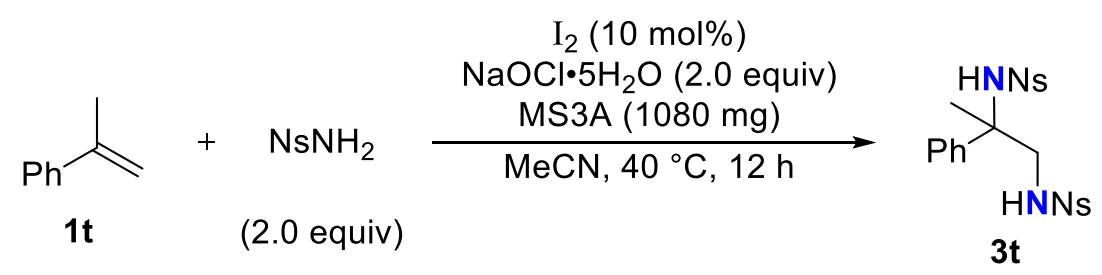

Following the general procedure (conditions I), isopropenylbenzene (1t: $58.2 \mathrm{mg}, 0.49 \mathrm{mmol}$ ), iodine (13.3 mg, $0.05 \mathrm{mmol}), 2$-nitrobenzenesulfonamide (202.8 mg, $1.00 \mathrm{mmol}$ ), sodium hypochlorite pentahydrate $(165.1 \mathrm{mg}, 1.00 \mathrm{mmol})$ and MS3A $(1080.5 \mathrm{mg})$ were stirred at $40{ }^{\circ} \mathrm{C}$ 
in $\mathrm{MeCN}$ for $12 \mathrm{~h}$. Washing the crude product with $\mathrm{Et}_{2} \mathrm{O}$ gave the product (3t: $184.0 \mathrm{mg}, 72 \%$ ) as a white solid. mp: $186.5-187.9{ }^{\circ} \mathrm{C}$; TLC: $\mathrm{R}_{\mathrm{f}}=0.30$ (hexane/EtOAc $\left.=1: 1\right) ;{ }^{1} \mathrm{H}$ NMR (400 MHz, acetone- $\left.d_{6}\right) \delta 8.14(\mathrm{~d}, J=7.2 \mathrm{~Hz}, 1 \mathrm{H}), 8.06-7.80(\mathrm{~m}, 4 \mathrm{H}), 7.73(\mathrm{dd}, J=7.2,7.2 \mathrm{~Hz}, 1 \mathrm{H})$, $7.48(\mathrm{dd}, J=8.0,8.0 \mathrm{~Hz}, 1 \mathrm{H}), 7.42-7.31(\mathrm{~m}, 3 \mathrm{H}), 7.20-7.00(\mathrm{~m}, 4 \mathrm{H}), 6.78$ (dd, $J=8.0,8.0 \mathrm{~Hz}$, $1 \mathrm{H}), 3.79(\mathrm{dd}, J=6.0,13.6 \mathrm{~Hz}, 1 \mathrm{H}), 3.53(\mathrm{dd}, J=7.2,13.6 \mathrm{~Hz}, 1 \mathrm{H}), 1.86(\mathrm{~s}, 3 \mathrm{H}) ;{ }^{13} \mathrm{C} \mathrm{NMR}$ (100 MHz, DMSO- $\left.d_{6}\right) \delta 148.9,148.3,141.7,135.6,135.0,134.2,134.0,133.8,133.1,131.3$, 131.0, 128.9, 128.5, 127.2, 125.9, 125.4, 62.2, 54.1, 24.8; IR (ATR) 3356, 3296, 3100, 3021, 2882, 1536, 1406, 1343, 1171, 1120, 1066, 1077, $729 \mathrm{~cm}^{-1}$; HRMS (FAB+): $\mathrm{m} / z$ calcd. for $\mathrm{C}_{21} \mathrm{H}_{21} \mathrm{~N}_{4} \mathrm{O}_{8} \mathrm{~S}_{2}\left([\mathrm{M}+\mathrm{H}]^{+}\right)$521.0801, found 521.0805.

\section{trans- $N, N^{\prime}$-(cyclopentane-1,2-diyl)bis(2-nitrobenzenesulfonamide) (4a)}

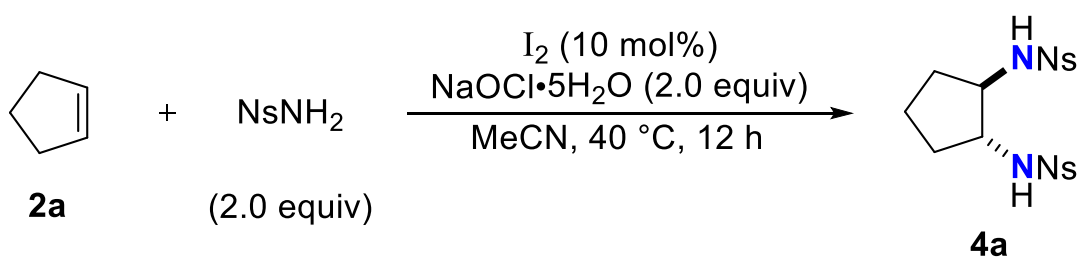

Following the general procedure (conditions I), cyclopentene (2a: $34.6 \mathrm{mg}, 0.51 \mathrm{mmol}$ ), iodine (13.0 mg, $0.05 \mathrm{mmol})$, 2-nitrobenzenesulfonamide (203.2 $\mathrm{mg}, 1.01 \mathrm{mmol}$ ) and sodium hypochlorite pentahydrate $(163.4 \mathrm{mg}, 0.99 \mathrm{mmol})$ were stirred at $40{ }^{\circ} \mathrm{C}$ in $\mathrm{MeCN}$ for $12 \mathrm{~h}$. Washing the crude product with $\mathrm{Et}_{2} \mathrm{O}$ gave the product (4a: $138.7 \mathrm{mg}, 58 \%$ ) as a white solid. mp: $212.7-213.5{ }^{\circ} \mathrm{C}$; TLC: $\mathrm{R}_{\mathrm{f}}=0.38$ (hexane/EtOAc $\left.=1: 1\right) ;{ }^{1} \mathrm{H}$ NMR (400 MHz, acetone- $\left.d_{6}\right)$

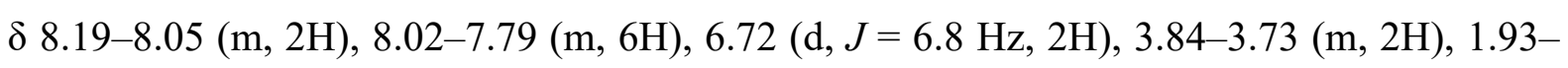
$1.81(\mathrm{~m}, 2 \mathrm{H}), 1.70-1.42(\mathrm{~m}, 4 \mathrm{H}) ;{ }^{13} \mathrm{C}$ NMR $\left(100 \mathrm{MHz}\right.$, acetone- $\left.d_{6}\right) \delta 148.9,134.9,134.8,133.6$, 131.4, 125.7, 60.8, 30.4, 20.6; IR (ATR) 1528, 1432, 1361, 1346, 1165, 1123, 1067, 895, 856, $788,741,731 \mathrm{~cm}^{-1}$; HRMS (CI+): $m / z$ calcd. for $\mathrm{C}_{17} \mathrm{H}_{19} \mathrm{~N}_{4} \mathrm{O}_{8} \mathrm{~S}_{2}\left([\mathrm{M}+\mathrm{H}]^{+}\right) 471.0644$, found 471.0648 .

trans- $N, N^{\prime}$-(cyclohexane-1,2-diyl)bis(2-nitrobenzenesulfonamide) (4b)

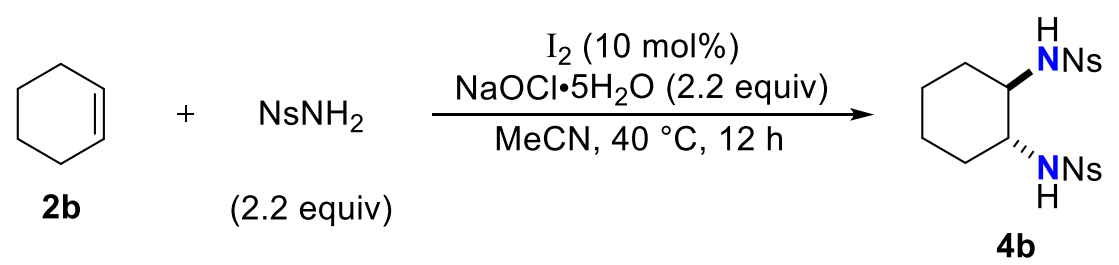

Following the general procedure (conditions I), cyclohexene (2b: $40.6 \mathrm{mg}, 0.49 \mathrm{mmol}$ ), iodine (12.7 mg, $0.05 \mathrm{mmol})$, 2-nitrobenzenesulfonamide (222.6 $\mathrm{mg}, 1.10 \mathrm{mmol}$ ) and sodium hypochlorite pentahydrate $(183.0 \mathrm{mg}, 1.11 \mathrm{mmol})$ were stirred at $40{ }^{\circ} \mathrm{C}$ in $\mathrm{MeCN}$ for $12 \mathrm{~h}$. 
Washing the crude product with $\mathrm{Et}_{2} \mathrm{O}$ gave the product (4b: $175.4 \mathrm{mg}, 73 \%$ ) as a white solid. mp: 209.6-211.6 ${ }^{\circ} \mathrm{C}$; TLC: $\mathrm{R}_{\mathrm{f}}=0.33$ (hexane/EtOAc $\left.=1: 1\right) ;{ }^{1} \mathrm{H}$ NMR (400 MHz, DMSO- $\left.d_{6}\right)$ 反 8.11-8.00 (m, 2H), 7.98-7.77 (m, 8H), 3.20-3.07 (m, 2H), 1.60-1.39 (m, 4H), 1.31-1.17 (m, 2H), 1.11-0.95 (m, 2H); ${ }^{13} \mathrm{C}$ NMR (100 MHz, DMSO- $\left.d_{6}\right) \delta 147.2,134.2,133.8,132.6,129.7$, 124.1, 56.1, 31.9, 23.5; IR (ATR) 3339, 3319, 3102, 2956, 2866, 1527, 1432, 1359, 1345, 1305, 1168, 1121, 1082, 1069, 959, 900, 858, 792, 741, $730 \mathrm{~cm}^{-1}$; HRMS (FAB+): $m / z$ calcd. for $\mathrm{C}_{18} \mathrm{H}_{21} \mathrm{~N}_{4} \mathrm{O}_{8} \mathrm{~S}_{2}\left([\mathrm{M}+\mathrm{H}]^{+}\right)$485.0801, found 485.0806.

\section{trans- $N, N^{\prime}$-(cyclohex-4-ene-1,2-diyl)bis(2-nitrobenzenesulfonamide) (4c)}

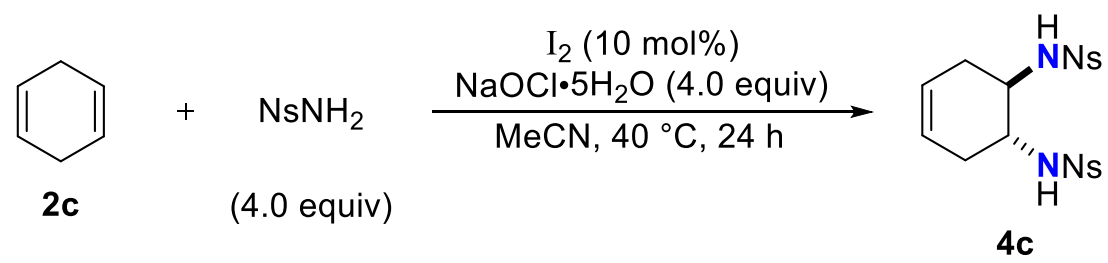

Following the general procedure (conditions I), 1,4-cyclohexadiene (2c: $38.6 \mathrm{mg}, 0.48 \mathrm{mmol}$ ), iodine (12.7 mg, $0.05 \mathrm{mmol})$, 2-nitrobenzenesulfonamide (405.4 mg, $2.01 \mathrm{mmol}$ ) and sodium hypochlorite pentahydrate $(331.0 \mathrm{mg}, 2.01 \mathrm{mmol})$ were stirred at $40{ }^{\circ} \mathrm{C}$ in $\mathrm{MeCN}$ for $24 \mathrm{~h}$. Purification by flash column chromatography on silica gel (hexane/EtOAc $=7: 3$ ) gave the product (4c: $138.0 \mathrm{mg}, 59 \%$ ) as a white solid. $\mathrm{mp}: 243.4-244.0{ }^{\circ} \mathrm{C}$; $\mathrm{TLC}: \mathrm{R}_{\mathrm{f}}=0.43$ (hexane/EtOAc = 1:1); ${ }^{1} \mathrm{H}$ NMR (400 MHz, DMSO- $\left.d_{6}\right) \delta 8.09-8.02(\mathrm{~m}, 2 \mathrm{H}), 7.98-7.90(\mathrm{~m}$, 4H), 7.89-7.78 (m, 4H), 5.43 (s, 2H), 3.45-3.33 (m, 2H), 2.17-2.02 (m, 4H); ${ }^{13} \mathrm{C}$ NMR (100 MHz, DMSO- $\left.d_{6}\right) \delta 147.3,134.0,133.8,132.6,129.7,124.3,124.1,52.2,30.8$; IR (ATR) 3319, $3015,3048,2841,1535,1443,1418,1364,1333,1159,1126,1074,1005,976,889,779,741$ $\mathrm{cm}^{-1}$; HRMS (FAB-): $m / z$ calcd. for $\mathrm{C}_{18} \mathrm{H}_{17} \mathrm{~N}_{4} \mathrm{O}_{8} \mathrm{~S}_{2}\left([\mathrm{M}-\mathrm{H}]^{-}\right)$481.0488, found 481.0486 .

trans- $N, N^{\prime}$-(cyclohex-3-ene-1,2-diyl)bis(2-nitrobenzenesulfonamide) (4d)

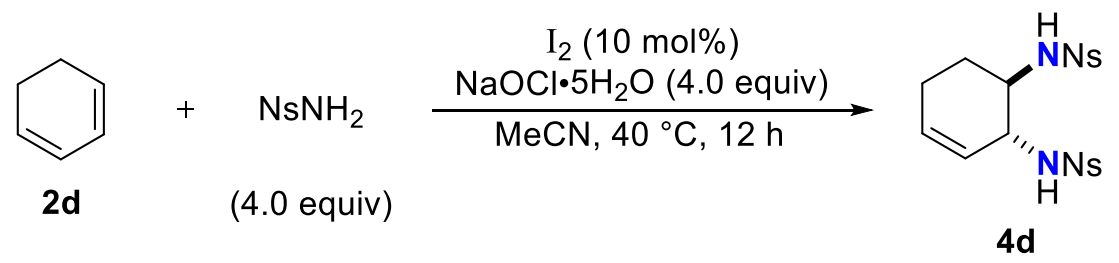

Following the general procedure (condition I), 1,3-cyclohexadiene (2d: $38.0 \mathrm{mg}, 0.47 \mathrm{mmol}$ ), iodine (13.1 mg, $0.05 \mathrm{mmol}$ ), 2-nitrobenzenesulfonamide (406.1 mg, $2.01 \mathrm{mmol}$ ) and sodium hypochlorite pentahydrate $(330.0 \mathrm{mg}, 2.01 \mathrm{mmol})$ were stirred at $40{ }^{\circ} \mathrm{C}$ in $\mathrm{MeCN}$ for $12 \mathrm{~h}$. Purification by flash column chromatography on silica gel $\left(\mathrm{CH}_{2} \mathrm{Cl}_{2} / \mathrm{MeOH}=19: 1\right)$ and washing with $\mathrm{Et}_{2} \mathrm{O}$ gave the product (4d: $135.3 \mathrm{mg}, 59 \%$ ) as a white solid. $\mathrm{mp}: 165.7-166.1{ }^{\circ} \mathrm{C}$; 
TLC: $\mathrm{R}_{\mathrm{f}}=0.40$ (hexane/EtOAc $\left.=1: 1\right) ;{ }^{1} \mathrm{H}$ NMR (400 MHz, DMSO- $\left.d_{6}\right) \delta 8.29(\mathrm{~d}, J=8.8 \mathrm{~Hz}$, 1H), 8.08-7.98 (m, 3H), 7.96-7.90 (m, 2H), 7.89-7.80 (m, 4H), 5.73-5.68 (m, 1H), 5.18-5.09 $(\mathrm{m}, 1 \mathrm{H}), 3.87-3.78(\mathrm{~m}, 1 \mathrm{H}), 2.08-1.85(\mathrm{~m}, 2 \mathrm{H}), 1.65-1.50(\mathrm{~m}, 2 \mathrm{H})\left(\mathrm{One} \mathrm{C}\left(\mathrm{sp}^{3}\right)-\mathrm{H}\right.$ signal is overlapped with $\mathrm{H}_{2} \mathrm{O}$ signal containing in DMSO- $d_{6}$.); ${ }^{13} \mathrm{C}$ NMR (100 MHz, DMSO- $\left.d_{6}\right) \delta$ 147.4, 147.3, 134.0, 133.9, 133.8, 133.7, 132.62, 132.60, 130.1, 129.7, 129.6, 125.5, 124.25, 124.23, 53.8, 53.5, 26.4, 22.6; IR (ATR) 3301, 2957, 1529, 1442, 1431, 1366, 1347, 1164, 1072, 855, $735 \mathrm{~cm}^{-1}$; HRMS (CI+): $m / z$ calcd. for $\mathrm{C}_{18} \mathrm{H}_{19} \mathrm{~N}_{4} \mathrm{O}_{8} \mathrm{~S}_{2}\left([\mathrm{M}+\mathrm{H}]^{+}\right) 483.0644$, found 483.0642 .

trans- $N, N^{\prime}$-(2,3-dihydro-1 $H$-indene-1,2-diyl)bis(2-nitrobenzenesulfonamide) (4e)

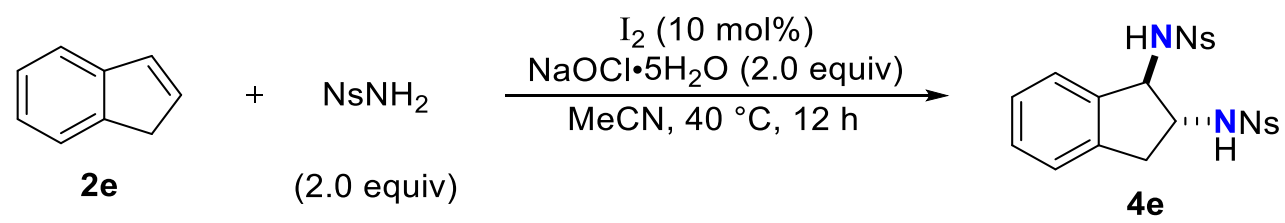

Following the general procedure (conditions I), indene (2e: $58.0 \mathrm{mg}, 0.50 \mathrm{mmol})$, iodine (12.8 $\mathrm{mg}, 0.05 \mathrm{mmol}$ ), 2-nitrobenzenesulfonamide (203.1 $\mathrm{mg}, 1.00 \mathrm{mmol}$ ) and sodium hypochlorite pentahydrate $(166.5 \mathrm{mg}, 1.01 \mathrm{mmol})$ were stirred at $40{ }^{\circ} \mathrm{C}$ in $\mathrm{MeCN}$ for $12 \mathrm{~h}$. Washing the crude product with $\mathrm{Et}_{2} \mathrm{O}$ gave the product (4e: $163.2 \mathrm{mg}, 63 \%$ ) as a white solid. mp: $206.8-$ $207.5{ }^{\circ} \mathrm{C}$; TLC: $\mathrm{R}_{\mathrm{f}}=0.48($ hexane/EtOAc $=1: 1) ;{ }^{1} \mathrm{H}$ NMR $\left(400 \mathrm{MHz}\right.$, DMSO- $\left.d_{6}\right) \delta 8.91(\mathrm{~d}, J$ $=8.8 \mathrm{~Hz}, 1 \mathrm{H}), 8.47(\mathrm{~d}, J=9.6 \mathrm{~Hz}, 1 \mathrm{H}), 8.17(\mathrm{~d}, J=7.2 \mathrm{~Hz}, 1 \mathrm{H}), 8.09-8.02(\mathrm{~m}, 1 \mathrm{H}), 8.01-7.95$ $(\mathrm{m}, 2 \mathrm{H}), 7.95-7.70(\mathrm{~m}, 4 \mathrm{H}), 7.29-7.01(\mathrm{~m}, 3 \mathrm{H}), 6.83(\mathrm{~d}, J=7.2 \mathrm{~Hz}, 1 \mathrm{H}), 4.93(\mathrm{dd}, J=8.8,8.8$ $\mathrm{Hz}, 1 \mathrm{H}), 4.07$ (dddd, $J=8.8,8.8,8.8,9.6 \mathrm{~Hz}, 1 \mathrm{H}), 2.78$ (dd, $J=8.0,15.6 \mathrm{~Hz}, 1 \mathrm{H}), 2.59$ (dd, $J$ $=8.8,15.6 \mathrm{~Hz}, 1 \mathrm{H}) ;{ }^{13} \mathrm{C}$ NMR $\left(100 \mathrm{MHz}, \mathrm{DMSO}-d_{6}\right) \delta 147.4,147.2,139.9,138.2,134.3,134.2$, $134.0,133.5,132.84,132.79,129.9,129.8,128.2$, 127.1, 124.6, 124.42, 124.39, 123.6, 62.8, 60.7, 35.7; IR (ATR) 3356, 3304, 1535, 1427, 1538, 1165, 1125, 1076, 854, 779, $733 \mathrm{~cm}^{-1}$; HRMS (FAB+): $m / z$ calcd. for $\mathrm{C}_{21} \mathrm{H}_{19} \mathrm{~N}_{4} \mathrm{O}_{8} \mathrm{~S}_{2}\left([\mathrm{M}+\mathrm{H}]^{+}\right)$519.0644, found 519.0645 . 
meso- $N, N^{\prime}$-(octane-4,5-diyl)bis(2-nitrobenzenesulfonamide) (4f)

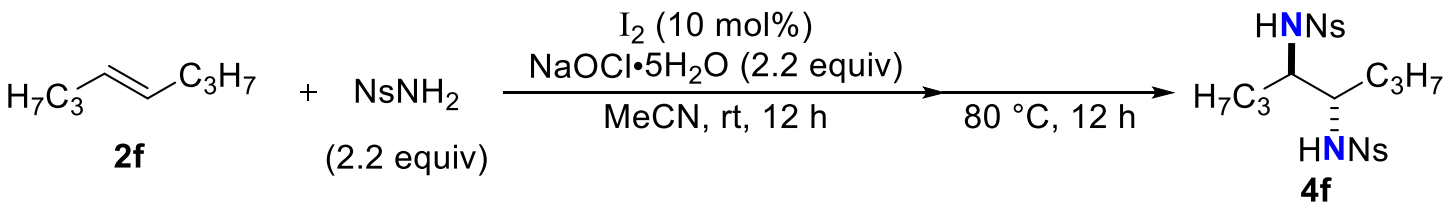

Following the general procedure (conditions II), trans-4-octene (2f: $54.6 \mathrm{mg}, 0.49 \mathrm{mmol})$, iodine (13.1 mg, $0.05 \mathrm{mmol}), 2$-nitrobenzenesulfonamide (223.0 mg, $1.10 \mathrm{mmol}$ ) and sodium hypochlorite pentahydrate $(181.7 \mathrm{mg}, 1.10 \mathrm{mmol})$ were stirred at room temperature in $\mathrm{MeCN}$ for $12 \mathrm{~h}$, then it warmed to $80{ }^{\circ} \mathrm{C}$ and stirred for $12 \mathrm{~h}$. Purification by flash column chromatography on silica gel $\left(\mathrm{CH}_{2} \mathrm{Cl}_{2} / \mathrm{MeOH}=19: 1\right)$ gave the product (4f: $\left.236.0 \mathrm{mg}, 94 \%\right)$ as a white solid. mp: $169.1-169.8^{\circ} \mathrm{C}$; TLC: $\mathrm{R}_{\mathrm{f}}=0.63$ (hexane/EtOAc $\left.=1: 1\right) ;{ }^{1} \mathrm{H}$ NMR $(400 \mathrm{MHz}$, $\left.\mathrm{CDCl}_{3}\right) \delta 8.08(\mathrm{dd}, J=3.2,6.4 \mathrm{~Hz}, 2 \mathrm{H}), 7.85(\mathrm{dd}, J=3.6,6.0 \mathrm{~Hz}, 2 \mathrm{H}), 7.81-7.68(\mathrm{~m}, 4 \mathrm{H}), 5.21$ $(\mathrm{d}, J=8.4 \mathrm{~Hz}, 2 \mathrm{H}), 3.58(\mathrm{dd}, J=8.4,11.6 \mathrm{~Hz}, 2 \mathrm{H}), 1.51-1.19(\mathrm{~m}, 6 \mathrm{H}), 1.13-0.99(\mathrm{~m}, 2 \mathrm{H})$, $0.77(\mathrm{t}, J=7.2 \mathrm{~Hz}, 6 \mathrm{H}) ;{ }^{13} \mathrm{C} \mathrm{NMR}\left(100 \mathrm{MHz}, \mathrm{CDCl}_{3}\right) \delta 147.6,134.4,133.6,133.0,130.6$, 125.3, 58.7, 33.0, 19.0, 13.5; IR (ATR) 3333, 3096, 3076, 2959, 2922, 2870, 1541, 1445, 1427 , 1366, 1341, 1325, 1244, 1159, 1120, 1074, 1036, 910, 854, $831 \mathrm{~cm}^{-1}$; HRMS (FAB+): $\mathrm{m} / z$ calcd. for $\mathrm{C}_{20} \mathrm{H}_{27} \mathrm{~N}_{4} \mathrm{O}_{8} \mathrm{~S}_{2}\left([\mathrm{M}+\mathrm{H}]^{+}\right)$515.1270, found 515.1275.

\section{$N, N^{\prime}$-((4R*, $\left.5 R^{*}\right)-$ octane-4,5-diyl)bis(2-nitrobenzenesulfonamide) (4g)}

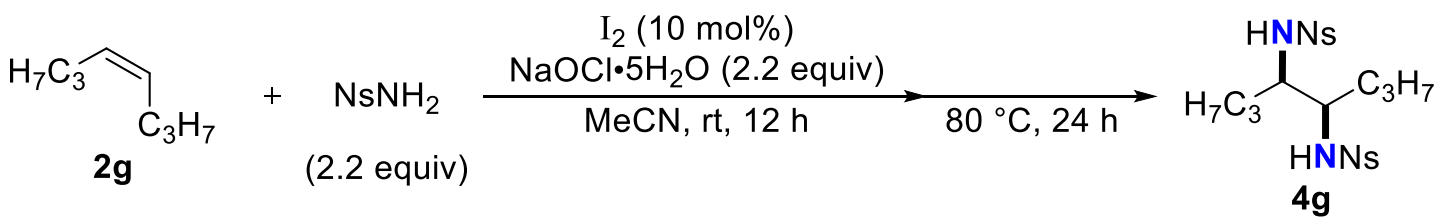

Following the general procedure (conditions II), cis-4-octene (2g: $54.6 \mathrm{mg}, 0.49 \mathrm{mmol})$, iodine (12.7 $\mathrm{mg}, 0.05 \mathrm{mmol})$, 2-nitrobenzenesulfonamide (221.2 $\mathrm{mg}, 1.09 \mathrm{mmol}$ ) and sodium hypochlorite pentahydrate $(181.5 \mathrm{mg}, 1.10 \mathrm{mmol})$ were stirred at room temperature in $\mathrm{MeCN}$ for $12 \mathrm{~h}$, then it warmed to $80{ }^{\circ} \mathrm{C}$ and stirred for $24 \mathrm{~h}$. Purification by flash column chromatography on silica gel (hexane/EtOAc $=7: 3$ ) gave the product $(4 \mathrm{~g}: 213.6 \mathrm{mg}, 85 \%)$ as a white solid. mp: $142.6-146.6{ }^{\circ} \mathrm{C}$; TLC: $\mathrm{R}_{\mathrm{f}}=0.45$ (hexane/EtOAc $\left.=1: 1\right) ;{ }^{1} \mathrm{H} \mathrm{NMR}(400 \mathrm{MHz}$, $\left.\mathrm{CDCl}_{3}\right) \delta 8.28-8.21(\mathrm{~m}, 2 \mathrm{H}), 7.94-7.85(\mathrm{~m}, 2 \mathrm{H}), 7.82-7.72(\mathrm{~m}, 4 \mathrm{H}), 5.17(\mathrm{~d}, J=6.4 \mathrm{~Hz}, 2 \mathrm{H})$, $3.58(\mathrm{dd}, J=4.0,6.4 \mathrm{~Hz}, 2 \mathrm{H}), 1.69-1.50\left(\mathrm{~m}, 2 \mathrm{H}\right.$, This signal is overlapped with $\mathrm{H}_{2} \mathrm{O}$ signal in $\mathrm{CDCl}_{3}$.), 1.29-1.12 (m, 4H), 0.99-0.81 (m, 2H), $0.65(\mathrm{t}, J=7.2 \mathrm{~Hz}, 6 \mathrm{H}) ;{ }^{13} \mathrm{C}$ NMR $(100 \mathrm{MHz}$, 
$\left.\mathrm{CDCl}_{3}\right) \delta 147.8,133.7,133.6,132.9,131.2,125.3,57.6,31.5,18.7,13.4$; IR (ATR) 3331, 3098, 2961, 2934, 2874, 1535, 1443, 1420, 1348, 1169, 1121, 1061, 1030, 957, 854, 785, $741 \mathrm{~cm}^{-1}$; HRMS (FAB+): $m / z$ calcd. for $\mathrm{C}_{20} \mathrm{H}_{27} \mathrm{~N}_{4} \mathrm{O}_{8} \mathrm{~S}_{2}\left([\mathrm{M}+\mathrm{H}]^{+}\right)$515.1270, found 515.1274.

\section{$N, N^{\prime}-\left(\left(2 R^{*}, 3 S^{*}\right)-\right.$ octane-2,3-diyl)bis(2-nitrobenzenesulfonamide) (4h)}

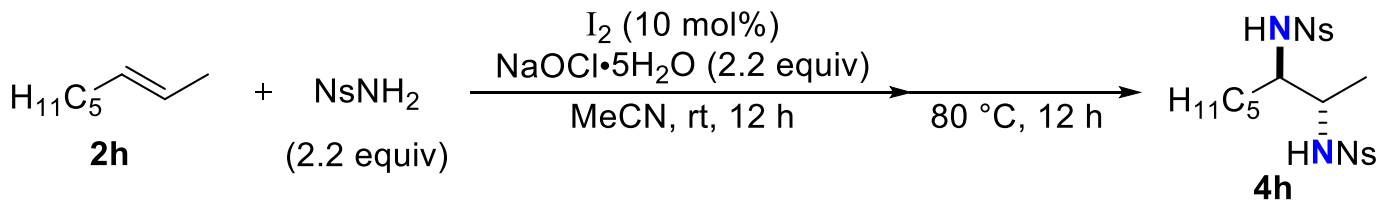

Following the general procedure (conditions II), trans-2-octene $(\mathbf{2 h}: 54.7 \mathrm{mg}, 0.49 \mathrm{mmol})$, iodine (13.3 mg, $0.05 \mathrm{mmol}$ ), 2-nitrobenzenesulfonamide (224.5 mg, $1.11 \mathrm{mmol}$ ) and sodium hypochlorite pentahydrate $(183.0 \mathrm{mg}, 1.11 \mathrm{mmol})$ were stirred at room temperature in $\mathrm{MeCN}$ for $12 \mathrm{~h}$, then it warmed to $80{ }^{\circ} \mathrm{C}$ and stirred for $12 \mathrm{~h}$. Purification by flash column chromatography on silica gel (hexane/EtOAc $=7: 3)$ gave the product $(\mathbf{4 h}: 193.2 \mathrm{mg}, 77 \%)$ as a white solid. mp: $132.2-133.3{ }^{\circ} \mathrm{C}$; TLC: $\mathrm{R}_{\mathrm{f}}=0.38$ (hexane/EtOAc $\left.=1: 1\right) ;{ }^{1} \mathrm{H} \mathrm{NMR}(400 \mathrm{MHz}$, $\left.\mathrm{CDCl}_{3}\right) \delta 8.18-8.05(\mathrm{~m}, 2 \mathrm{H}), 7.91-7.82(\mathrm{~m}, 2 \mathrm{H}), 7.82-7.69(\mathrm{~m}, 4 \mathrm{H}), 5.35(\mathrm{~d}, J=8.0 \mathrm{~Hz}, 1 \mathrm{H})$, $5.25(\mathrm{~d}, J=8.8 \mathrm{~Hz}, 1 \mathrm{H}), 3.72-3.61(\mathrm{~m}, 1 \mathrm{H}), 3.59-3.50(\mathrm{~m}, 1 \mathrm{H}), 1.53-1.43(\mathrm{~m}, 1 \mathrm{H}), 1.42-1.31$ (m, 1H), 1.31-1.19 (m, 1H), 1.19-0.95 (m, 8H), $0.77(\mathrm{t}, J=6.8 \mathrm{~Hz}, 3 \mathrm{H}) ;{ }^{13} \mathrm{C}$ NMR $(100 \mathrm{MHz}$, $\left.\mathrm{CDCl}_{3}\right) \delta 147.8,147.6,134.35,134.30,133.7,133.1,133.0,130.7,125.5,125.4,59.6,54.0$, $31.3,31.1,25.4,22.3,16.5,13.8$ (Two sp ${ }^{2}$ signals were not observed because of overlapping.); IR (ATR) 3312, 3094, 2955, 2934, 2868, 1545, 1435, 1339, 1163, 1117, 1078, 961, 853, 779, 745, $729 \mathrm{~cm}^{-1}$; HRMS (FAB +$): m / z$ calcd. for $\mathrm{C}_{20} \mathrm{H}_{27} \mathrm{~N}_{4} \mathrm{O}_{8} \mathrm{~S}_{2}\left([\mathrm{M}+\mathrm{H}]^{+}\right)$515.1270, found 515.1276 .

\section{$N, N^{\prime}-\left(\left(2 R^{*}, 3 R^{*}\right)-o c t a n e-2,3-d i y l\right) b i s(2-n i t r o b e n z e n e s u l f o n a m i d e)(4 i)$}

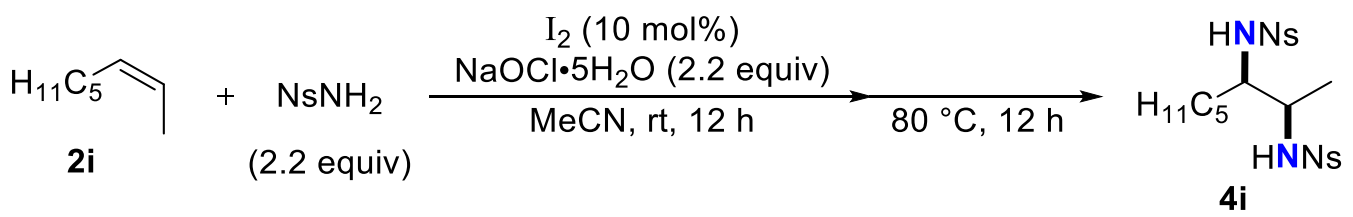

Following the general procedure (conditions II), cis-2-octene (2i: $55.6 \mathrm{mg}, 0.50 \mathrm{mmol}$ ), iodine (12.9 mg, $0.05 \mathrm{mmol})$, 2-nitrobenzenesulfonamide $(223.8 \mathrm{mg}, 1.11 \mathrm{mmol})$ and sodium hypochlorite pentahydrate $(183.3 \mathrm{mg}, 1.11 \mathrm{mmol})$ were stirred at room temperature in MeCN for $12 \mathrm{~h}$, then it warmed to $80{ }^{\circ} \mathrm{C}$ and stirred for $12 \mathrm{~h}$. Purification by flash column chromatography on silica gel $\left(\mathrm{CH}_{2} \mathrm{Cl}_{2} / \mathrm{MeOH}=19: 1\right)$ gave the product (4i: $\left.230.6 \mathrm{mg}, 91 \%\right)$ as 
a pale yellow solid. mp: $95.1-95.9{ }^{\circ} \mathrm{C}$; TLC: $\mathrm{R}_{\mathrm{f}}=0.25$ (hexane/EtOAc $\left.=1: 1\right) ;{ }^{1} \mathrm{H}$ NMR $(400$ $\left.\mathrm{MHz}, \mathrm{CDCl}_{3}\right) \delta 8.27-8.18(\mathrm{~m}, 2 \mathrm{H}), 7.95-7.87(\mathrm{~m}, 2 \mathrm{H}), 7.85-7.72(\mathrm{~m}, 4 \mathrm{H}), 5.20(\mathrm{~d}, J=6.8 \mathrm{~Hz}$, 2H), 3.78-3.68 (m, 1H), 3.55-3.45 (m, 1H), 1.69-1.58 (m, 1H), 1.36-1.22 (m, 1H), 1.15-0.80 $(\mathrm{m}, 9 \mathrm{H}), 0.71(\mathrm{t}, J=6.8 \mathrm{~Hz}, 3 \mathrm{H}) ;{ }^{13} \mathrm{C} \mathrm{NMR}\left(100 \mathrm{MHz}, \mathrm{CDCl}_{3}\right) \delta 147.9,147.8,133.83,133.76$, 133.7, 133.5, 132.99, 132.96, 131.3, 130.9, 125.5, 125.4, 58.7, 53.3, 31.0, 29.7, 25.2 , 22.3 , 16.4, 13.8; IR (ATR) 3319, 3096, 2953, 2932, 2868, 1537, 1427, 1344, 1163, 1119, 1059, 961, 854, 787, 743, $727 \mathrm{~cm}^{-1}$; HRMS (FAB+): $m / z$ calcd. for $\mathrm{C}_{20} \mathrm{H}_{27} \mathrm{~N}_{4} \mathrm{O}_{8} \mathrm{~S}_{2}\left([\mathrm{M}+\mathrm{H}]^{+}\right)$515.1270, found 515.1275 .

\section{$N, N^{\prime}-\left(\left(1 R^{*}, 2 S^{*}\right)-1-p h e n y l p r o p a n e-1,2-d i y l\right) b i s(2-n i t r o b e n z e n e s u l f o n a m i d e)(4 j)$}

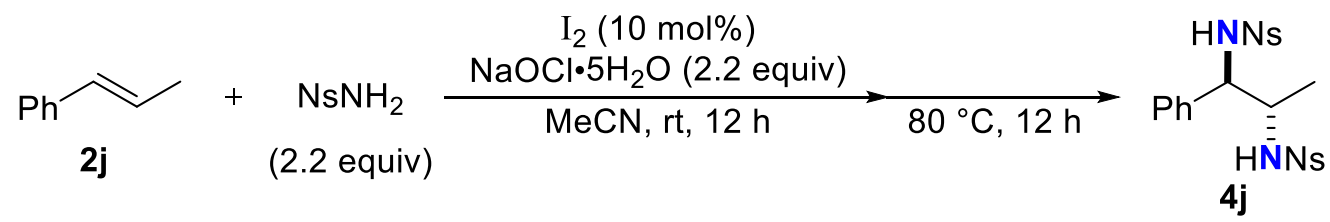

Following the general procedure (conditions II), trans-1-phenylpropene (2j: $58.3 \mathrm{mg}, 0.49$ $\mathrm{mmol}$ ), iodine (13.0 mg, $0.05 \mathrm{mmol})$, 2-nitrobenzenesulfonamide (221.6 mg, $1.10 \mathrm{mmol}$ ) and sodium hypochlorite pentahydrate $(181.1 \mathrm{mg}, 1.10 \mathrm{mmol})$ were stirred at room temperature in $\mathrm{MeCN}$ for $12 \mathrm{~h}$, then it warmed to $80{ }^{\circ} \mathrm{C}$ and stirred for $12 \mathrm{~h}$. Purification by flash column chromatography on silica gel $\left(\mathrm{CH}_{2} \mathrm{Cl}_{2} / \mathrm{MeOH}=19: 1\right)$ gave the product $(4 \mathbf{j}: 163.7 \mathrm{mg}, 64 \%)$ as a white solid. mp: $193.1-194.2{ }^{\circ} \mathrm{C}$; TLC: $\mathrm{R}_{\mathrm{f}}=0.33$ (hexane/EtOAc $\left.=1: 1\right) ;{ }^{1} \mathrm{H} \mathrm{NMR}(400 \mathrm{MHz}$, DMSO-d $\left.d_{6}\right) \delta .62(\mathrm{~d}, J=10.0 \mathrm{~Hz}, 1 \mathrm{H}), 7.99(\mathrm{~d}, J=8.8 \mathrm{~Hz}, 1 \mathrm{H}), 7.82-7.69(\mathrm{~m}, 3 \mathrm{H}), 7.68-7.58$ $(\mathrm{m}, 3 \mathrm{H}), 7.58-7.49(\mathrm{~m}, 2 \mathrm{H}), 7.12-7.05(\mathrm{~m}, 2 \mathrm{H}), 6.92-6.80(\mathrm{~m}, 3 \mathrm{H}), 4.32(\mathrm{dd}, J=10.0,10.0$ $\mathrm{Hz}, 1 \mathrm{H}), 3.80-3.68(\mathrm{~m}, 1 \mathrm{H}), 1.10(\mathrm{~d}, J=6.4 \mathrm{~Hz}, 3 \mathrm{H}) ;{ }^{13} \mathrm{C}$ NMR $\left(100 \mathrm{MHz}, \mathrm{DMSO}-d_{6}\right) \delta 146.9$, 146.8, 138.3, 133.7, 133.6, 133.4, 132.7, 132.6, 132.1, 129.8, 129.3, 127.4, 127.0, 124.3, 123.9, 61.8, 54.0, 19.1 (One sp ${ }^{2}$ signal was not observed because of overlapping.); IR (ATR) 3325, 1530, 1447, 1366, 1348, 1165, 1057, 910, 854, 779, $727 \mathrm{~cm}^{-1}$; HRMS (FAB-): $m / z$ calcd. for $\mathrm{C}_{21} \mathrm{H}_{19} \mathrm{~N}_{4} \mathrm{O}_{8} \mathrm{~S}_{2}\left([\mathrm{M}-\mathrm{H}]^{-}\right)$519.0644, found 519.0647 .

\section{$N, N^{\prime}$-((1 $\left.\left.R^{*}, 2 R^{*}\right)-1-p h e n y l p r o p a n e-1,2-d i y l\right) b i s(2-n i t r o b e n z e n e s u l f o n a m i d e)(4 \mathrm{k})$}

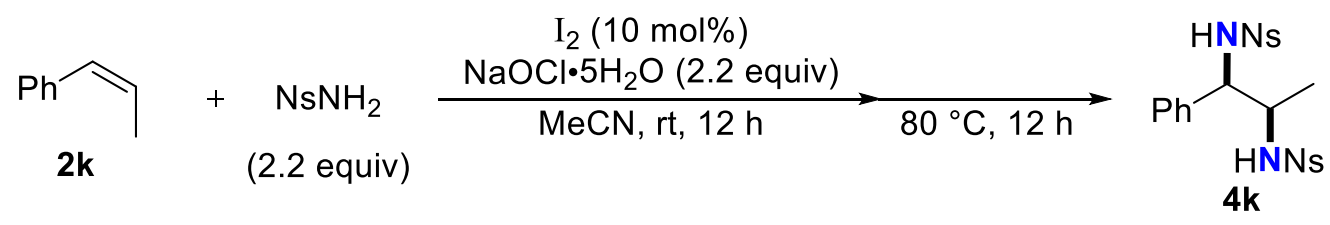


Following the general procedure (conditions II), cis-1-phenylpropene (2k: $60.7 \mathrm{mg}, 0.51$ $\mathrm{mmol})$, iodine (13.0 mg, $0.05 \mathrm{mmol})$, 2-nitrobenzenesulfonamide (221.0 mg, $1.09 \mathrm{mmol})$ and sodium hypochlorite pentahydrate $(183.0 \mathrm{mg}, 1.11 \mathrm{mmol})$ were stirred at room temperature in $\mathrm{MeCN}$ for $12 \mathrm{~h}$, then it warmed to $80^{\circ} \mathrm{C}$ and stirred for $12 \mathrm{~h}$. Purification by flash column chromatography on silica gel (hexane/EtOAc $=1: 1)$ gave the product $(4 \mathrm{k}: 165.2 \mathrm{mg}, 62 \%)$ as a white solid. mp: $175.1-175.9^{\circ} \mathrm{C}$; TLC: $\mathrm{R}_{\mathrm{f}}=0.33$ (hexane/EtOAc $\left.=1: 1\right) ;{ }^{1} \mathrm{H} \mathrm{NMR}(400 \mathrm{MHz}$, DMSO- $\left.d_{6}\right) \delta 8.45(\mathrm{~d}, J=10.0 \mathrm{~Hz}, 1 \mathrm{H}), 7.91(\mathrm{~d}, J=9.6 \mathrm{~Hz}, 1 \mathrm{H}), 7.88-7.60(\mathrm{~m}, 7 \mathrm{H}), 7.58-7.48$ $(\mathrm{m}, 1 \mathrm{H}), 7.15(\mathrm{~d}, J=2.0 \mathrm{~Hz}, 2 \mathrm{H}), 6.99-6.89$ (m, 3H), $4.58(\mathrm{dd}, J=5.0,10.0 \mathrm{~Hz}, 1 \mathrm{H}), 3.82-$ $3.68(\mathrm{~m}, 1 \mathrm{H}), 0.97(\mathrm{~d}, J=6.4 \mathrm{~Hz}, 3 \mathrm{H}) ;{ }^{13} \mathrm{C}$ NMR $\left(100 \mathrm{MHz}, \mathrm{DMSO}-d_{6}\right) \delta 146.9,137.8,133.9$, 133.8, 133.4, 132.8, 132.6, 132.2, 129.9, 129.4, 127.5, 127.0, 126.9, 124.5, 124.1, 61.6, 54.7, 19.0 (One sp ${ }^{2}$ signal was not observed because of overlapping.); IR (ATR) 3327, 3300, 3103, $1535,1441,1360,1341,1163,1125,1059,986,920,854 \mathrm{~cm}^{-1}$; HRMS (FAB-): $\mathrm{m} / z$ calcd. for $\mathrm{C}_{21} \mathrm{H}_{19} \mathrm{~N}_{4} \mathrm{O}_{8} \mathrm{~S}_{2}\left([\mathrm{M}-\mathrm{H}]^{-}\right)$519.0644, found 519.0644 .

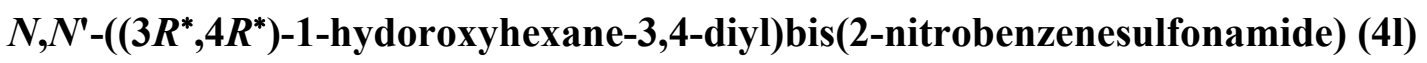

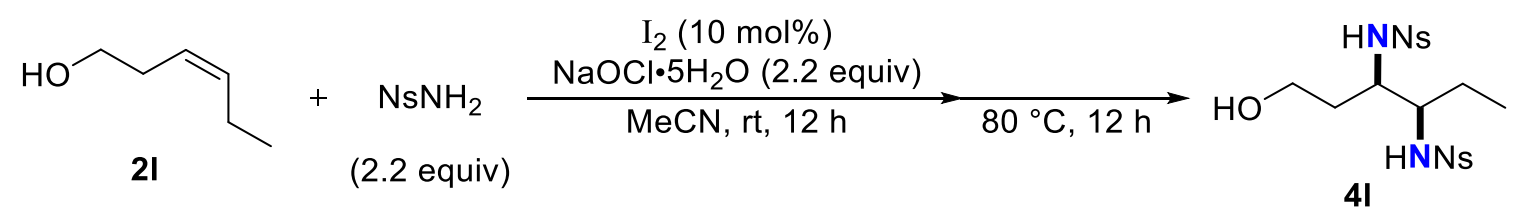

Following the general procedure (conditions II), cis-3-hexene-1-ol (2l: $49.5 \mathrm{mg}, 0.49 \mathrm{mmol}$ ), iodine (13.6 mg, $0.05 \mathrm{mmol})$, 2-nitrobenzenesulfonamide (221.5 mg, $1.09 \mathrm{mmol}$ ) and sodium hypochlorite pentahydrate $(182.0 \mathrm{mg}, 1.11 \mathrm{mmol})$ were stirred at room temperature in MeCN for $12 \mathrm{~h}$, then it warmed to $80{ }^{\circ} \mathrm{C}$ and stirred for $12 \mathrm{~h}$. Purification by flash column chromatography on silica gel $\left(\mathrm{CH}_{2} \mathrm{Cl}_{2} / \mathrm{MeOH}=19: 1\right)$ gave the product (4l: $\left.224.6 \mathrm{mg}, 90 \%\right)$ as a white solid. mp: $183.2-184.0{ }^{\circ} \mathrm{C}$; TLC: $\mathrm{R}_{\mathrm{f}}=0.60$ (EtOAc); ${ }^{1} \mathrm{H}$ NMR (400 MHz, DMSO- $d_{6}$ ) $\delta 8.12-7.78(\mathrm{~m}, 10 \mathrm{H}), 4.30(\mathrm{t}, J=4.8 \mathrm{~Hz}, 1 \mathrm{H}), 3.52-3.41(\mathrm{~m}, 1 \mathrm{H}), 3.29-3.19(\mathrm{~m}, 1 \mathrm{H}), 3.18-$ $3.09(\mathrm{~m}, 1 \mathrm{H}), 3.06-2.93(\mathrm{~m}, 1 \mathrm{H}), 1.75-1.63(\mathrm{~m}, 1 \mathrm{H}), 1.62-1.50(\mathrm{~m}, 1 \mathrm{H}), 1.49-1.32(\mathrm{~m}, 1 \mathrm{H})$, $1.29-1.12(\mathrm{~m}, 1 \mathrm{H}), 0.47(\mathrm{t}, J=7.2 \mathrm{~Hz}, 3 \mathrm{H}) ;{ }^{13} \mathrm{C} \mathrm{NMR}\left(100 \mathrm{MHz}, \mathrm{DMSO}-d_{6}\right) \delta 147.2,147.1$, 134.2, 133.1, 133.0, 132.5, 132.4, 130.2, 130.0, 124.5, 58.7, 57.3, 53.9, 31.8, 21.5, 10.4 (Two $\mathrm{sp}^{2}$ signals were not observed because of overlapping.); IR (ATR) 3557, 3310, 2961, 2932, 2886, 1535, 1420, 1366, 1337, 1181, 1125, 1061, 854, $782 \mathrm{~cm}^{-1}$; HRMS (FAB+): $\mathrm{m} / z$ calcd. for $\mathrm{C}_{18} \mathrm{H}_{23} \mathrm{~N}_{4} \mathrm{O}_{9} \mathrm{~S}_{2}\left([\mathrm{M}+\mathrm{H}]^{+}\right)$503.0906, found 503.0900. 


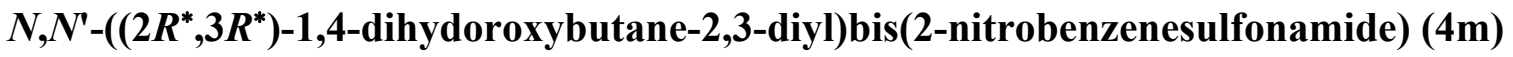

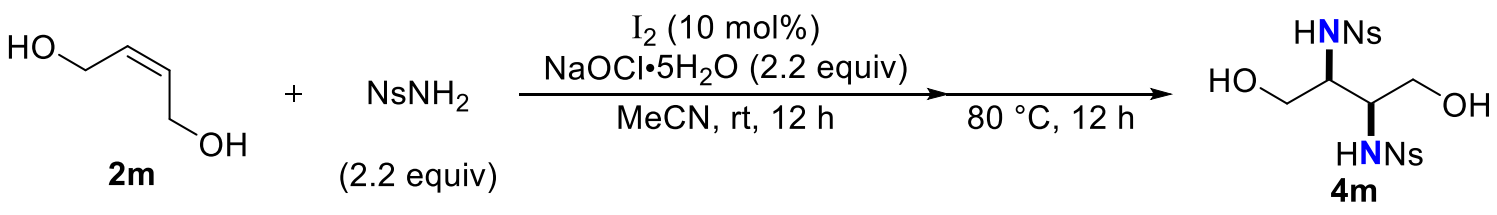

Following the general procedure (conditions II), cis-2-butene-1,4-diol (2m: $43.0 \mathrm{mg}, 0.49$ $\mathrm{mmol}$ ), iodine (12.7 mg, $0.05 \mathrm{mmol}), 2$-nitrobenzenesulfonamide (223.8 mg, $1.11 \mathrm{mmol}$ ) and sodium hypochlorite pentahydrate $(182.8 \mathrm{mg}, 1.11 \mathrm{mmol})$ were stirred at room temperature in $\mathrm{MeCN}$ for $12 \mathrm{~h}$, then it warmed to $80{ }^{\circ} \mathrm{C}$ and stirred for $12 \mathrm{~h}$. Purification by flash column chromatography on silica gel (EtOAc) gave the product (4m: $162.6 \mathrm{mg}, 68 \%$ ) as a yellow solid. mp: $178.8-179.7^{\circ} \mathrm{C}$; TLC: $\mathrm{R}_{\mathrm{f}}=0.30$ (EtOAc); ${ }^{1} \mathrm{H}$ NMR $\left(400 \mathrm{MHz}, \mathrm{DMSO}-d_{6}\right) \delta 8.10-8.01(\mathrm{~m}$, 2H), 8.00-7.92 (m, 2H), 7.91-7.75 (m, 4H), $7.66(\mathrm{~d}, J=8.8 \mathrm{~Hz}, 2 \mathrm{H}), 5.57$ (t, $J=4.8 \mathrm{~Hz}, 2 \mathrm{H})$, $3.62-3.51(\mathrm{~m}, 2 \mathrm{H}), 3.38-3.30\left(\mathrm{~m}, 2 \mathrm{H}\right.$, This signal is overlapped with $\mathrm{H}_{2} \mathrm{O}$ signal in DMSO- $\left.d_{6}\right)$, $3.28-3.17(\mathrm{~m}, 2 \mathrm{H}) ;{ }^{13} \mathrm{C}$ NMR (100 MHz, DMSO- $\left.d_{6}\right) \delta 147.2,133.9,133.6,132.6,130.2,124.4$, 60.2, 55.8; IR (ATR) 3566, 3350, 3100, 1535, 1425, 1354, 1165, 1061, 953, 858, 743, $729 \mathrm{~cm}^{-}$ 1; HRMS (FAB+): $m / z$ calcd. for $\mathrm{C}_{16} \mathrm{H}_{19} \mathrm{~N}_{4} \mathrm{O}_{10} \mathrm{~S}_{2}\left([\mathrm{M}+\mathrm{H}]^{+}\right) 491.0543$, found 491.0549 .

\section{$N, N^{\prime}-\left(\left(3 R^{*}, 4 R^{*}\right)-1-((t e r t-b u t y l d i m e t h y l s i l y l) o x y) h e x a n e-3,4-d i y l\right) b i s(2-$ nitrobenzenesulfonamide) (4n)}

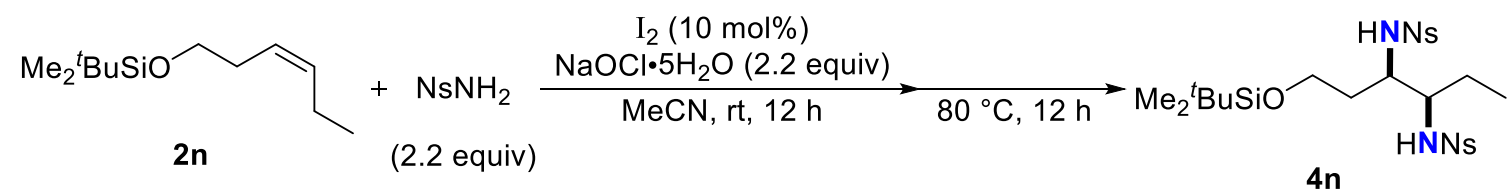

Following the general procedure (conditions II), cis-(hex-3-en-1-yloxy)tert-butyl dimethylsilane (2n: $105.4 \mathrm{mg}, \quad 0.49 \mathrm{mmol})$, iodine (13.2 mg, $0.05 \mathrm{mmol})$, 2nitrobenzenesulfonamide $(222.3 \mathrm{mg}, 1.10 \mathrm{mmol})$ and sodium hypochlorite pentahydrate $(182.5$ $\mathrm{mg}, 1.11 \mathrm{mmol}$ ) were stirred at room temperature in $\mathrm{MeCN}$ for $12 \mathrm{~h}$, then it warmed to $80{ }^{\circ} \mathrm{C}$ and stirred for $12 \mathrm{~h}$. Purification by flash column chromatography on silica gel (hexane/EtOAc $=7: 3$ ) gave the product (4n: $240.2 \mathrm{mg}, 79 \%$ ) as a white solid. $\mathrm{mp}: 149.9-150.6{ }^{\circ} \mathrm{C}$; $\mathrm{TLC}: \mathrm{R}_{\mathrm{f}}=$ 0.53 (hexane/EtOAc = 1:1); ${ }^{1} \mathrm{H}$ NMR $\left(400 \mathrm{MHz}, \mathrm{CDCl}_{3}\right) \delta 8.25-8.15(\mathrm{~m}, 2 \mathrm{H}), 7.92-7.87(\mathrm{~m}$, 2H), 7.81-7.72 (m, 4H), 5.46 (d, $J=7.2 \mathrm{~Hz}, 1 \mathrm{H}), 5.33$ (d, $J=7.6 \mathrm{~Hz}, 1 \mathrm{H}), 3.90-3.79$ (m, 1H), 3.62-3.50 (m, 2H), 3.47-3.39 (m, 1H), 1.93-1.82 (m, 1H), 1.81-1.69 (m, 1H), 1.51-1.41 (m, $1 \mathrm{H}), 1.40-1.27(\mathrm{~m}, 1 \mathrm{H}), 0.84(\mathrm{~s}, 9 \mathrm{H}), 0.67$ (t, $J=7.6 \mathrm{~Hz}, 3 \mathrm{H}),-0.02(\mathrm{~s}, 3 \mathrm{H}),-0.06(\mathrm{~s}, 3 \mathrm{H}) ;{ }^{13} \mathrm{C}$ NMR $\left(100 \mathrm{MHz}, \mathrm{CDCl}_{3}\right) \delta 147.9,147.8,135.1,134.1,133.9,133.7,133.6,132.9,131.2,130.7$, $125.33,125.27,58.9,54.6,33.6,25.9,23.8,18.2,10.3,-0.03,-5.50$ (One $\mathrm{sp}^{3}$ signal was not 
observed because of overlapping.); IR (ATR) 3348, 3316, 3096, 2957, 2932, 2882, 2853, 1537 , 1429, 1356, 1165, 1101, 1088, 1022, 961, 918, 781, $744 \mathrm{~cm}^{-1}$; HRMS (FAB+): $\mathrm{m} / z$ calcd. for $\mathrm{C}_{24} \mathrm{H}_{37} \mathrm{~N}_{4} \mathrm{O}_{9} \mathrm{~S}_{2} \mathrm{Si}\left([\mathrm{M}+\mathrm{H}]^{+}\right)$617.1771, found 617.1767.

\section{$N, N^{\prime}$-(2-methylhexane-2,3-diyl)bis(2-nitrobenzenesulfonamide) (4o)}

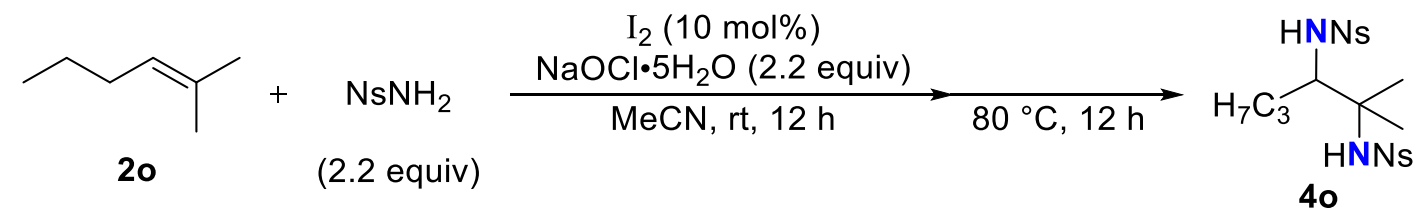

Following the general procedure (conditions II), 2-methyl-2-hexene (2o: $52.4 \mathrm{mg}, 0.53 \mathrm{mmol}$ ), iodine (13.0 mg, $0.05 \mathrm{mmol}), 2$-nitrobenzenesulfonamide (222.7 mg, $1.10 \mathrm{mmol}$ ) and sodium hypochlorite pentahydrate $(179.8 \mathrm{mg}, 1.09 \mathrm{mmol})$ were stirred at room temperature in $\mathrm{MeCN}$ for $12 \mathrm{~h}$, then it warmed to $80{ }^{\circ} \mathrm{C}$ and stirred for $12 \mathrm{~h}$. Purification by flash column chromatography on silica gel $\left(\mathrm{CH}_{2} \mathrm{Cl}_{2} / \mathrm{MeOH}=19: 1\right)$ gave the product (4o: $\left.150.4 \mathrm{mg}, 56 \%\right)$ as a pale yellow solid. mp: $78.3-79.5{ }^{\circ} \mathrm{C}$; TLC: $\mathrm{R}_{\mathrm{f}}=0.43$ (hexane/EtOAc $\left.=1: 1\right) ;{ }^{1} \mathrm{H}$ NMR $(400$ $\left.\mathrm{MHz}, \mathrm{CDCl}_{3}\right) \delta 8.28-8.19(\mathrm{~m}, 1 \mathrm{H}), 8.18-8.10(\mathrm{~m}, 1 \mathrm{H}), 7.95-7.81(\mathrm{~m}, 2 \mathrm{H}), 7.80-7.68(\mathrm{~m}, 4 \mathrm{H})$, $5.71(\mathrm{~d}, J=9.6 \mathrm{~Hz}, 1 \mathrm{H}), 5.32$ (s, 1H), 3.67 (ddd, $J=2.2,9.6,9.6 \mathrm{~Hz}, 1 \mathrm{H}), 1.78-1.61(\mathrm{~m}, 1 \mathrm{H})$, 1.55-1.41 (m, 1H), $1.31(\mathrm{~s}, 3 \mathrm{H}), 1.26(\mathrm{~s}, 3 \mathrm{H}), 1.24-1.09(\mathrm{~m}, 1 \mathrm{H}), 1.04-0.88(\mathrm{~m}, 1 \mathrm{H}), 0.75$ (t, $J=7.2 \mathrm{~Hz}, 3 \mathrm{H}) ;{ }^{13} \mathrm{C} \mathrm{NMR}\left(100 \mathrm{MHz}, \mathrm{CDCl}_{3}\right) \delta 147.8,147.6,136.4,135.6,133.5,133.3,133.1$, 133.0, 130.8, 130.1 125.4, 125.3, 63.0, 60.9, 32.7, 26.6, 24.6, 19.7, 13.7; IR (ATR) 3325, 3102, 2967, 2876, 1537, 1420, 1360, 1163, 1123, 1059, 999, 854, 781, $741 \mathrm{~cm}^{-1}$; HRMS (FAB+): $m / z$ calcd. for $\mathrm{C}_{19} \mathrm{H}_{25} \mathrm{~N}_{4} \mathrm{O}_{8} \mathrm{~S}_{2}\left([\mathrm{M}+\mathrm{H}]^{+}\right)$501.1114, found 501.1118 .

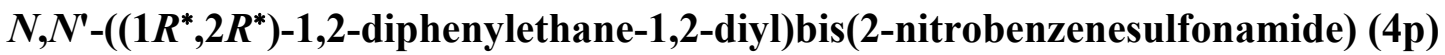

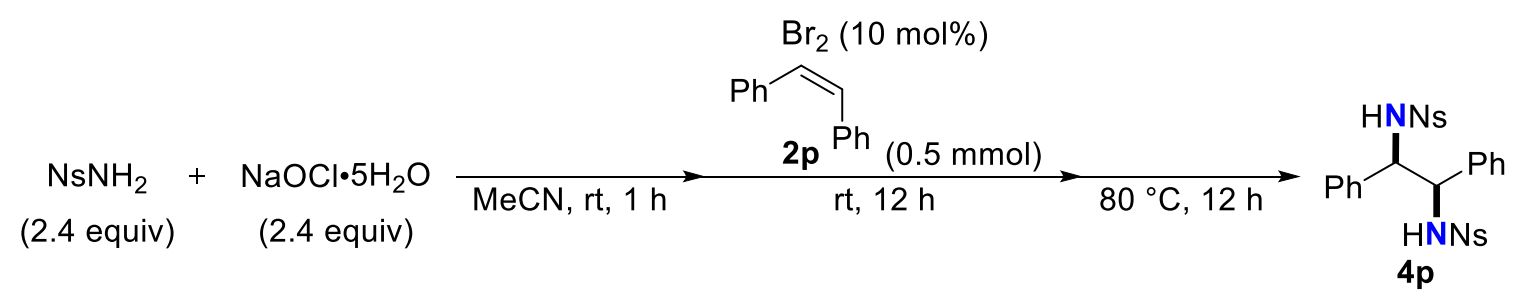

A solution of 2-nitrobenzenesulfonamide $(245.5 \mathrm{mg}, 1.21 \mathrm{mmol})$ and sodium hypochlorite pentahydrate $(198.0 \mathrm{mg}, 1.20 \mathrm{mmol})$ under a nitrogen atmosphere in $\mathrm{MeCN}(3 \mathrm{~mL})$ was stirred at room temperature for $1 \mathrm{~h}$. After stirring, bromine $(2.56 \mu \mathrm{L}, 0.05 \mathrm{mmol})$ and cis-stilbene (2p: $87.6 \mathrm{mg}, 0.49 \mathrm{mmol}$ ) were added to the stirring solution and stirred at room temperature for 12 $\mathrm{h}$, then it warmed to $80^{\circ} \mathrm{C}$ and stirred for $12 \mathrm{~h}$. Following the general procedure (conditions 
II), crude product was obtained. Purification by flash column chromatography on silica gel $\left(\mathrm{CH}_{2} \mathrm{Cl}_{2} / \mathrm{MeOH}=19: 1\right)$ and washing with $\mathrm{Et}_{2} \mathrm{O}$ gave the product (4p: $\left.100.1 \mathrm{mg}, 35 \%\right)$ as a white solid. The diastereomeric ratio of the purified product was determined by ${ }^{1} \mathrm{H}$ NMR analysis. mp: $115.9-116.5{ }^{\circ} \mathrm{C}$; TLC: $\mathrm{R}_{\mathrm{f}}=0.53$ (hexane/EtOAc $\left.=1: 1\right) ;{ }^{1} \mathrm{H}$ NMR $(400 \mathrm{MHz}$, DMSO- $\left.d_{6}\right) \delta 8.64(\mathrm{~d}, J=8.4 \mathrm{~Hz}, 2 \mathrm{H}), 7.73(\mathrm{~d}, J=8.0 \mathrm{~Hz}, 2 \mathrm{H}), 7.63-7.55(\mathrm{~m}, 2 \mathrm{H}), 7.49-7.29$ $(\mathrm{m}, 4 \mathrm{H}), 7.23(\mathrm{~d}, J=6.8 \mathrm{~Hz}, 4 \mathrm{H}), 7.02-6.86(\mathrm{~m}, 6 \mathrm{H}), 4.88(\mathrm{~d}, J=8.4 \mathrm{~Hz}, 2 \mathrm{H}) ;{ }^{13} \mathrm{C} \mathrm{NMR}(100$ MHz, DMSO-d $d_{6} \delta 146.5,138.0,133.5,132.7,132.3,129.6,127.5,127.01,126.96,124.1$, 62.5; IR (ATR) 3333, 1530, 1362, 1341, 1161, 1125, 1059, 934, 854, $827 \mathrm{~cm}^{-1}$; HRMS (FAB-): $m / z$ calcd. for $\mathrm{C}_{26} \mathrm{H}_{21} \mathrm{~N}_{4} \mathrm{O}_{8} \mathrm{~S}_{2}\left([\mathrm{M}-\mathrm{H}]^{-}\right)$581.0801, found 581.0808 .

\section{syn-1,2-Diamination}

\section{di-tert-butyl $\left(3 \mathrm{a} R^{*}, 6 \mathrm{a} S^{*}\right)$-cyclopenta[ $[c][1,2,5]$ thiadiazolidine-1,3-dicarboxylate 2,2- dioxide (5a)}

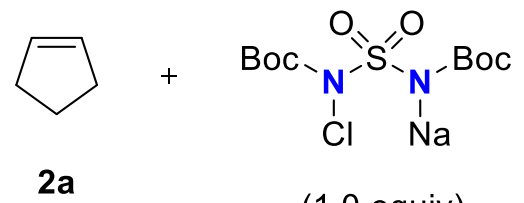

(1.0 equiv)
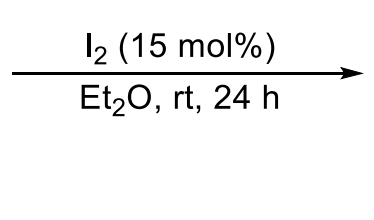

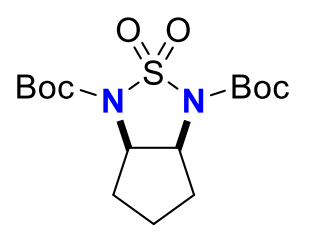

$5 \mathbf{a}$

(0.5 mmol scale)

Following the general procedure (syn-1,2-Diamination), chloramine-BBS (177.3 $\mathrm{mg}, 0.50$ mmol), cyclopentene (2a: $34.6 \mathrm{mg}, 0.51 \mathrm{mmol})$ and $\mathrm{I}_{2}(19.1 \mathrm{mg}, 0.075 \mathrm{mmol})$ were stirred at room temperature in $\mathrm{Et}_{2} \mathrm{O}$ for $24 \mathrm{~h}$. Purification by flash column chromatography on silica gel (hexane/EtOAc $=9: 1)$ gave the product $(\mathbf{5 a}: 126.1 \mathrm{mg}, 70 \%)$ as a white solid. mp: 138.8$139.5{ }^{\circ} \mathrm{C}$; TLC: $\mathrm{R}_{\mathrm{f}}=0.45$ (hexane/EtOAc $\left.=7: 3\right) ;{ }^{1} \mathrm{H}$ NMR $\left(400 \mathrm{MHz}, \mathrm{CDCl}_{3}\right) \delta 4.46(\mathrm{ddd}, J$ $=5.6,5.6,11.2 \mathrm{~Hz}, 2 \mathrm{H}), 2.08-1.98(\mathrm{~m}, 4 \mathrm{H}), 1.97-1.83(\mathrm{~m}, 1 \mathrm{H}), 1.72-1.60(\mathrm{~m}, 1 \mathrm{H}), 1.56(\mathrm{~s}$, $18 \mathrm{H}) ;{ }^{13} \mathrm{C} \mathrm{NMR}\left(100 \mathrm{MHz}, \mathrm{CDCl}_{3}\right) \delta 149.0,85.2,56.5,33.6,27.9,22.4$; IR (ATR) 2982, 2940, 2876, 1732, 1705, 1366, 1346, 1275, 1252, 1167, 1142, 1090, 1024, 874, 837, $766 \mathrm{~cm}^{-1}$; HRMS $(\mathrm{FAB}+): m / z$ calcd. for $\mathrm{C}_{15} \mathrm{H}_{27} \mathrm{~N}_{2} \mathrm{O}_{6} \mathrm{~S}\left([\mathrm{M}+\mathrm{H}]^{+}\right) 363.1590$, found 363.1584 .

(gram scale) 
Following the general procedure (syn-1,2-Diamination), chloramine-BBS (1770.0 $\mathrm{mg}, 5.0$ mmol), cyclopentene (2a: $340.6 \mathrm{mg}, 5.7 \mathrm{mmol})$ and $\mathrm{I}_{2}(191.0 \mathrm{mg}, 0.75 \mathrm{mmol})$ were stirred at room temperature in $\mathrm{Et}_{2} \mathrm{O}$ for $72 \mathrm{~h}$. Purification by flash column chromatography on silica gel (hexane/EtOAc $=9: 1)$ gave the product (5a: $1260.0 \mathrm{mg}, 70 \%)$ as a white solid.

\section{di-tert-butyl (3a $\left.R^{*}, 7 \mathrm{a} S^{*}\right)$-cyclohexa[c] $[1,2,5]$ thiadiazolidine-1,3-dicarboxylate 2,2-dioxide} (5b)

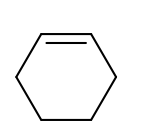

2b<smiles>CC(C)(C)OC(=O)N([18O])S(=O)(=O)N(Cl)C(=O)O</smiles>

(1.0 equiv)

(1)

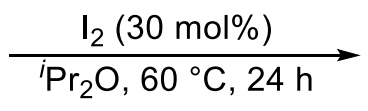

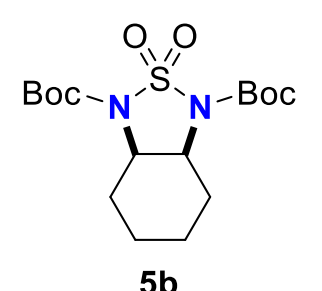

$5 b$

Following the general procedure (syn-1,2-Diamination), chloramine-BBS (176.9 mg, 0.50 mmol), cyclohexene (2b: $41.1 \mathrm{mg}, 0.5 \mathrm{mmol})$ and $\mathrm{I}_{2}(38.6 \mathrm{mg}, 0.152 \mathrm{mmol})$ were stirred at $60{ }^{\circ} \mathrm{C}$ in ${ }^{i} \operatorname{Pr}_{2} \mathrm{O}$ for $24 \mathrm{~h}$. Purification by flash column chromatography on silica gel (hexane/EtOAc $=95: 5)$ gave the product $(\mathbf{5 b}: 67.7 \mathrm{mg}, 36 \%)$ as a white solid. $\mathrm{mp:}$ : 121.8$122.5{ }^{\circ} \mathrm{C}$; TLC: $\mathrm{R}_{\mathrm{f}}=0.23$ (hexane/EtOAc $\left.=7: 3\right) ;{ }^{1} \mathrm{H}$ NMR $\left(400 \mathrm{MHz}, \mathrm{CDCl}_{3}\right) \delta 4.13(\mathrm{ddd}, J$ $=4.2,4.2,8.4 \mathrm{~Hz}, 2 \mathrm{H}), 2.38-2.25(\mathrm{~m}, 2 \mathrm{H}), 1.91-1.81(\mathrm{~m}, 2 \mathrm{H}), 1.56(\mathrm{~s}, 18 \mathrm{H}), 1.50-1.33(\mathrm{~m}$, 2H) (Two $\mathrm{C}\left(\mathrm{sp}^{3}\right)-\mathrm{H}$ signals are overlapped with the ${ }^{t} \mathrm{Bu}$ signal of the $\mathbf{5 b}$ ); ${ }^{13} \mathrm{C}$ NMR (100 $\left.\mathrm{MHz}, \mathrm{CDCl}_{3}\right) \delta 149.4,85.2,54.0,28.0,26.5,20.9$; IR (ATR) 2982, 2940, 2860, 1732, 1726, 1454, 1346, 1328, 1248, 1173, 1144, 999, 846, $762 \mathrm{~cm}^{-1}$; HRMS (FAB-): $m / z$ calcd. for $\mathrm{C}_{16} \mathrm{H}_{27} \mathrm{~N}_{2} \mathrm{O}_{6} \mathrm{~S}\left([\mathrm{M}-\mathrm{H}]^{-}\right) 375.1590$, found 375.1595 .

\section{di-tert-butyl $\left(3 \mathrm{a} R^{*}, 8 \mathrm{a} S^{*}\right)$-cyclohepta[c][1,2,5]thiadiazolidine-1,3-dicarboxylate 2,2- dioxide (5q)}

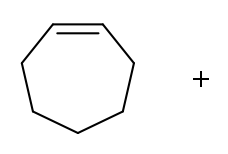

$2 q$<smiles>O=C(O)c1ccccc1</smiles>

(1.0 equiv)

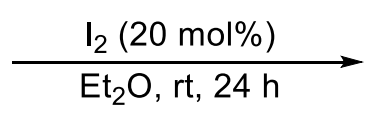<smiles>CC(C)(C)OC(=O)N1C2CCCCCC2N(C(=O)OCc2ccccc2)S1(=O)=O</smiles>

$5 \mathbf{q}$

Following the general procedure (syn-1,2-Diamination), chloramine-BBS (177.7 $\mathrm{mg}, 0.50$ mmol), cycloheptene (2q: $45.5 \mathrm{mg}, 0.47 \mathrm{mmol})$ and $\mathrm{I}_{2}(26.2 \mathrm{mg}, 0.103 \mathrm{mmol})$ were stirred at room temperature in $\mathrm{Et}_{2} \mathrm{O}$ for $24 \mathrm{~h}$. Purification by flash column chromatography on silica gel (hexane/EtOAc $=95: 5)$ gave the product $(\mathbf{5 q}: 109.5 \mathrm{mg}, 59 \%)$ as a pale yellow solid. $\mathrm{mp}$ : 150.9-151.4 ${ }^{\circ} \mathrm{C}$; TLC: $\mathrm{R}_{\mathrm{f}}=0.13$ (hexane/EtOAc $\left.=9: 1\right) ;{ }^{1} \mathrm{H}$ NMR $\left(400 \mathrm{MHz}, \mathrm{CDCl}_{3}\right) \delta 4.28$ 
(ddd, $J=3.6,3.6,8.8 \mathrm{~Hz}, 2 \mathrm{H}), 2.26-2.19(\mathrm{~m}, 2 \mathrm{H}), 1.95-1.73(\mathrm{~m}, 5 \mathrm{H}), 1.55(\mathrm{~s}, 18 \mathrm{H}), 1.43-$ $1.25(\mathrm{~m}, 3 \mathrm{H}) ;{ }^{13} \mathrm{C} \mathrm{NMR}\left(100 \mathrm{MHz}, \mathrm{CDCl}_{3}\right) \delta 149.1,85.1,57.1,30.1,29.4,27.9,24.9$; IR (ATR) 2994, 2982, 2930, 1728, 1476, 1449, 1344, 1275, 1252, 1175, 1136, 1098, 1069, 1051, 901, 822, 773, $756 \mathrm{~cm}^{-1}$; HRMS (FAB+): $m / z$ calcd. for $\mathrm{C}_{17} \mathrm{H}_{31} \mathrm{~N}_{2} \mathrm{O}_{6} \mathrm{~S}\left([\mathrm{M}+\mathrm{H}]^{+}\right) 391.1903$, found 391.1906 .

\section{di-tert-butyl $\left(3 \mathrm{a} R^{*}, 8 \mathrm{~b} S^{*}\right)$-inda[1,2-c][1,2,5] thiadiazolidine-1,3-dicarboxylate 2,2-dioxide} (5e)<smiles>C1=Cc2ccccc2C1</smiles>

$2 e$<smiles>O=C(OCc1ccccc1)N([AlH2])S(=O)(=O)N(Cl)C(=O)O</smiles>

(1.0 equiv)

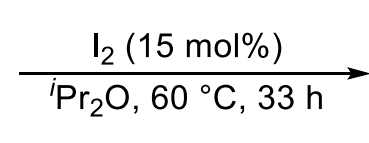<smiles>CC(C)(C)OC(=O)N1C2c3ccccc3C[C@H]2N(C(=O)OCc2ccccc2)S1(=O)=O</smiles>

$5 e$

Following the general procedure (syn-1,2-Diamination), chloramine-BBS (178.0 mg, 0.50 $\mathrm{mmol}$ ), indene (2e: $57.5 \mathrm{mg}, 0.50 \mathrm{mmol})$ and $\mathrm{I}_{2}(19.9 \mathrm{mg}, 0.078 \mathrm{mmol})$ were stirred at $60{ }^{\circ} \mathrm{C}$ in ${ }^{i} \operatorname{Pr}_{2} \mathrm{O}$ for $33 \mathrm{~h}$. Purification by flash column chromatography on silica gel (hexane/EtOAc $=$ 9:1) gave the product (5e: $146.3 \mathrm{mg}, 72 \%$ ) as a white solid. $\mathrm{mp}: 147.3-148.1^{\circ} \mathrm{C}$; $\mathrm{TLC}$ : $\mathrm{R}_{\mathrm{f}}=$ $0.50($ hexane/EtOAc $=7: 3) ;{ }^{1} \mathrm{H}$ NMR $\left(400 \mathrm{MHz}, \mathrm{CDCl}_{3}\right) \delta 7.60(\mathrm{~d}, J=7.2 \mathrm{~Hz}, 1 \mathrm{H}), 7.39-7.20$ $(\mathrm{m}, 3 \mathrm{H}), 5.62(\mathrm{~d}, J=7.6 \mathrm{~Hz}, 1 \mathrm{H}), 4.95-4.84(\mathrm{~m}, 1 \mathrm{H}), 3.45(\mathrm{dd}, J=6.8,16.8 \mathrm{~Hz}, 1 \mathrm{H}), 3.35$ (dd, $J=3.2,16.8 \mathrm{~Hz}, 1 \mathrm{H}), 1.60(\mathrm{~s}, 9 \mathrm{H}), 1.57(\mathrm{~s}, 9 \mathrm{H}) ;{ }^{13} \mathrm{C} \mathrm{NMR}\left(100 \mathrm{MHz}, \mathrm{CDCl}_{3}\right) \delta 149.4,149.3$, 140.1, 138.1, 129.8, 127.7, 126.6, 125.1, 85.60, 85.58, 59.7, 56.2, 38.0, 28.0 (One sp ${ }^{3}$ signal was not observed because of overlapping.); IR (ATR) 2978, 1736, 1722, 1477, 1460, 1352, $1329,1275,1256,1173,1144,1105,1090,1057,949,848,818,804,756,735 \mathrm{~cm}^{-1}$; HRMS $(\mathrm{FAB}+): m / z$ calcd. for $\mathrm{C}_{19} \mathrm{H}_{27} \mathrm{~N}_{2} \mathrm{O}_{6} \mathrm{~S}\left([\mathrm{M}+\mathrm{H}]^{+}\right) 411.1590$, found 411.1587 . 

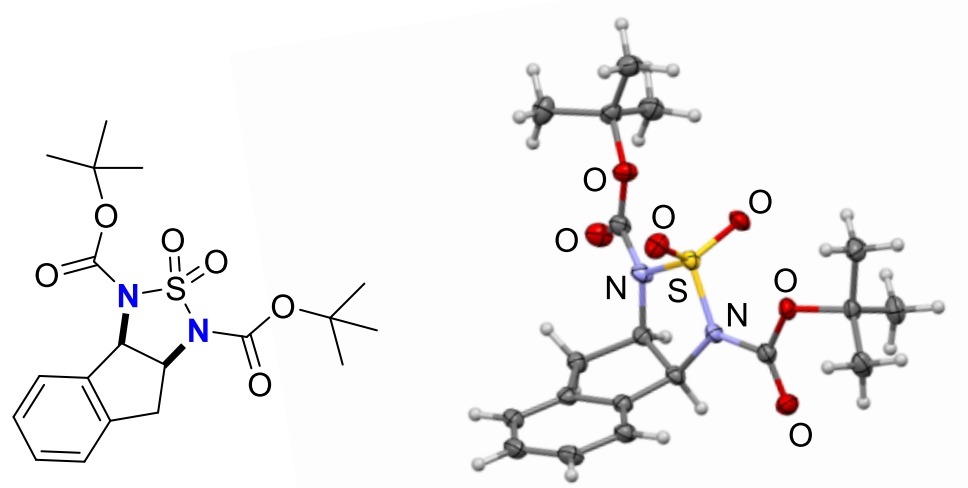

Fig. S9. Crystal structure of 5e. CCDC 1919391 contains the supplementary crystallographic data. The data can be obtained free of charge from The Cambridge Crystallographic Data Centre via www.ccdc.cam.ac.uk/data request/cif

Table S8. Crystallographic data of $5 e$

$\begin{array}{ll}\text { Empirical Formula } & \mathrm{C}_{19} \mathrm{H}_{26} \mathrm{~N}_{2} \mathrm{O}_{6} \mathrm{~S} \\ \text { Formula Weight } & 410.48 \\ \text { Crystal System } & \text { monoclinic } \\ \text { Space Group } & \mathrm{P} 2{ }_{1} / \mathrm{c}(\# 14) \\ \text { Unit cell dimensions } & \mathrm{a}=22.2901(2) \AA \\ & \mathrm{b}=10.02490(8) \AA \\ & \mathrm{c}=19.29780(18) \AA \\ V & \beta=107.4000(10) \mathrm{o} \\ Z & 4114.88(7) \AA^{3} \\ \text { Density (calculated) } & 8 \\ \text { Absorption coefficient } & 1.325 \mathrm{~g} / \mathrm{cm}^{3} \\ \text { R1 (I>2.00 }(\mathrm{I})) & 1.725 \mathrm{~cm}^{-1} \\ \text { wR2 (All reflections) } & 0.0351 \\ \text { Crystal size } & 0.0968 \\ \text { Goodness of Fit Indicator } & 0.100 \times 0.150 \times 0.200 \mathrm{~mm} \\ \text { No. of Reflections Measured } & 1.049 \\ & \text { Total: } 23285 \\ & \mathrm{Unique}: 8174\left(\mathrm{R}_{\mathrm{int}}=0.0276\right)\end{array}$


<smiles>Brc1ccc2c(c1)CC=C2</smiles>

$2 \mathbf{r}$<smiles>O=C(O)c1ccccc1</smiles>

(1.5 equiv)
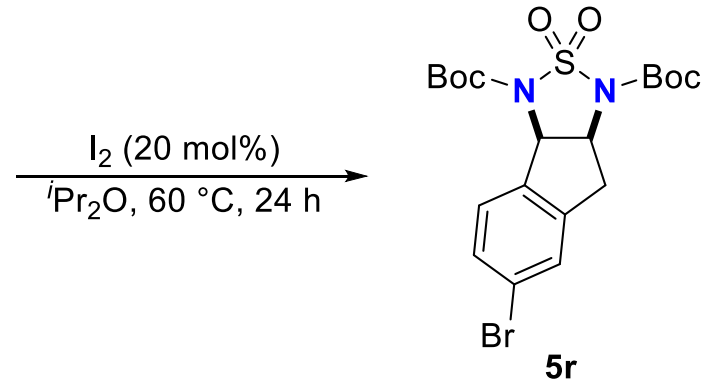

Following the general procedure (syn-1,2-Diamination), chloramine-BBS (265.1 $\mathrm{mg}, 0.75$ mmol), 6-bromo- $1 H$-indene (2r: $93.7 \mathrm{mg}, 0.48 \mathrm{mmol})$ and $\mathrm{I}_{2}(26.5 \mathrm{mg}, 0.10 \mathrm{mmol})$ were stirred at $60{ }^{\circ} \mathrm{C}$ in ${ }^{i} \operatorname{Pr}_{2} \mathrm{O}$ for $24 \mathrm{~h}$. Purification by flash column chromatography on silica gel (hexane/EtOAc $=9: 1)$ gave the product $(5 \mathrm{r}: 143.9 \mathrm{mg}, 61 \%)$ as a white solid. $\mathrm{mp}: 146.5-$ $147.0{ }^{\circ} \mathrm{C}$; TLC: $\mathrm{R}_{\mathrm{f}}=0.58$ (hexane/EtOAc $\left.=7: 3\right) ;{ }^{1} \mathrm{H}$ NMR $\left(400 \mathrm{MHz}, \mathrm{CDCl}_{3}\right) \delta 7.48(\mathrm{~d}, J=$ $8.8 \mathrm{~Hz}, 1 \mathrm{H}), 7.43-7.37(\mathrm{~m}, 2 \mathrm{H}), 5.55(\mathrm{~d}, J=8.0 \mathrm{~Hz}, 1 \mathrm{H}), 4.92-4.86(\mathrm{~m}, 1 \mathrm{H}), 3.43(\mathrm{dd}, J=6.4$, $17.6 \mathrm{~Hz}, 1 \mathrm{H}), 3.34$ (dd, $J=4.0,17.6 \mathrm{~Hz}, 1 \mathrm{H}), 1.59$ (s, 9H), 1.57 (s, 9H); ${ }^{13} \mathrm{C}$ NMR $(100 \mathrm{MHz}$, $\left.\mathrm{CDCl}_{3}\right) \delta 149.4,149.2,142.4,137.2,131.0,128.3,128.1,124.0,85.9,85.8,59.1,56.2,37.8$, 27.9 (One sp ${ }^{3}$ signal was not observed because of overlapping.); IR (ATR) 2986, 2941, 1732, 1474, 1350, 1279, 1254, 1144, 1099, 1057, 841, $826 \mathrm{~cm}^{-1}$; HRMS (FAB+): $\mathrm{m} / z$ calcd. for $\mathrm{C}_{19} \mathrm{H}_{26} \mathrm{BrN}_{2} \mathrm{O}_{6} \mathrm{~S}\left([\mathrm{M}+\mathrm{H}]^{+}\right) 489.0695$, found 489.0685 .

di-tert-butyl

$\left(3 \mathrm{a} R^{*}, 9 \mathrm{~b} S^{*}\right)-4,5-d i h y d r o n a p h t h a[1,2-c][1,2,5]$ thiadiazolidine-1,3dicarboxylate 2,2-dioxide $(5 \mathrm{~s})$

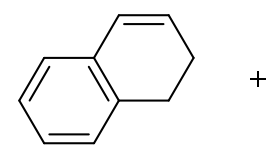

2s<smiles>O=C(O)c1ccccc1</smiles>

(1.5 equiv)
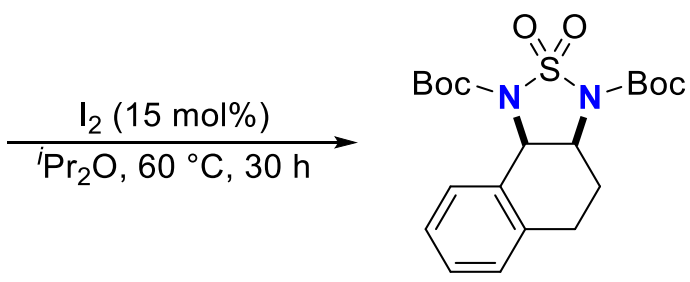

5s

Following the general procedure (syn-1,2-Diamination), chloramine-BBS (264.6 $\mathrm{mg}, 0.75$ $\mathrm{mmol}$ ), 1,2-dihydronaphthalene (2s: $64.8 \mathrm{mg}, 0.50 \mathrm{mmol})$ and $\mathrm{I}_{2}(19.3 \mathrm{mg}, 0.075 \mathrm{mmol})$ were stirred at $60{ }^{\circ} \mathrm{C}$ in ${ }^{i} \operatorname{Pr}_{2} \mathrm{O}$ for $30 \mathrm{~h}$. Purification by flash column chromatography on silica gel (hexane/EtOAc $=9: 1)$ gave the product $(\mathbf{5 s}: 170.1 \mathrm{mg}, 81 \%)$ as a white solid. $\mathrm{mp}: 108.0$ $108.9^{\circ} \mathrm{C}$; TLC: $\mathrm{R}_{\mathrm{f}}=0.58($ hexane/EtOAc $=7: 3) ;{ }^{1} \mathrm{H}$ NMR $\left(400 \mathrm{MHz}, \mathrm{CDCl}_{3}\right) \delta 7.51-7.43(\mathrm{~m}$, 
1H), 7.29-7.20 (m, 2H), 7.17-7.10 (m, 1H), 5.47 (d, J=7.2 Hz, 1H), 4.68-4.61 (m, 1H), 3.12$2.91(\mathrm{~m}, 1 \mathrm{H}), 2.69-2.59(\mathrm{~m}, 1 \mathrm{H}), 2.58-2.48(\mathrm{~m}, 1 \mathrm{H}), 2.02-1.90(\mathrm{~m}, 1 \mathrm{H}), 1.562(\mathrm{~s}, 9 \mathrm{H}), 1.559$ $(\mathrm{s}, 9 \mathrm{H}) ;{ }^{13} \mathrm{C} \mathrm{NMR}\left(100 \mathrm{MHz}, \mathrm{CDCl}_{3}\right) \delta 149.6,149.5,138.1,131.8,129.4,128.4,128.2,126.8$, 85.6, 85.4, 53.6, 53.4, 28.0, 27.1, 24.3 (One $\mathrm{sp}^{3}$ signal was not observed because of overlapping.); IR (ATR) 2984, 2932, 1736, 1456, 1364, 1344, 1323, 1250, 1144, 1153, 1053, 841, 795, $743 \mathrm{~cm}^{-1}$; HRMS (FAB+): $m / z$ calcd. for $\mathrm{C}_{20} \mathrm{H}_{29} \mathrm{~N}_{2} \mathrm{O}_{6} \mathrm{~S}\left([\mathrm{M}+\mathrm{H}]^{+}\right) 425.1746$, found 425.1741 .

\section{di-tert-butyl $\left(3 \mathrm{a} S^{*}, 9 \mathrm{~b} R^{*}\right)-7-m e t h o x y-3 \mathrm{a}, 4,5,9 \mathrm{~b}$-tetrahydronaphtho[1,2- \\ c][1,2,5]thiadiazole-1,3-}

\section{dicarboxylate 2,2-dioxide (2t)}

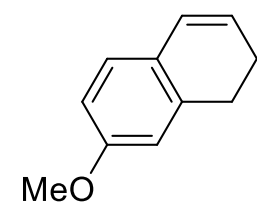

$2 t$

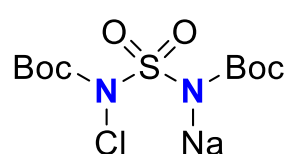

(1.0 equiv)

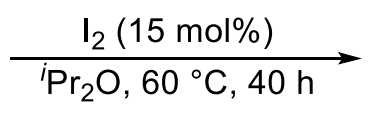

$\mathrm{MeO}$

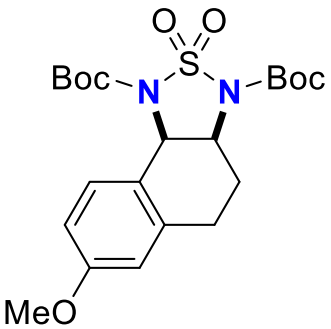

$5 \mathbf{t}$

Following the general procedure (syn-1,2-Diamination), chloramine-BBS (178.3 mg, 0.71 mmol), 7-methoxy-1,2-dihydronaphthalene (2s: $78.6 \mathrm{mg}, 0.49 \mathrm{mmol})$ and $\mathrm{I}_{2}(19.2 \mathrm{mg}, 0.075$ mmol) were stirred at $60{ }^{\circ} \mathrm{C}$ in ${ }^{i} \operatorname{Pr}_{2} \mathrm{O}$ for $40 \mathrm{~h}$. Purification by flash column chromatography on silica gel (hexane/EtOAc $=95: 5)$ gave the product $(\mathbf{5 s}: 111.9 \mathrm{mg}, 50 \%)$ as a white solid. $\mathrm{mp}$ : 140.8-141.8 ${ }^{\circ} \mathrm{C}$; TLC: $\mathrm{R}_{\mathrm{f}}=0.13$ (hexane/EtOAc = 9:1); ${ }^{1} \mathrm{H}$ NMR $\left(400 \mathrm{MHz}, \mathrm{CDCl}_{3}\right) \delta 7.41$ $(\mathrm{d}, J=8.8 \mathrm{~Hz}, 1 \mathrm{H}), 6.76(\mathrm{dd}, J=2.8,8.8 \mathrm{~Hz}, 1 \mathrm{H}), 6.65(\mathrm{~d},=2.4 \mathrm{~Hz}, 1 \mathrm{H}), 4.61(\mathrm{~m}, 1 \mathrm{H}), 3.79$ $(\mathrm{s}, 3 \mathrm{H}), 2.97(\mathrm{~m}, 1 \mathrm{H}), 2.56,(\mathrm{~m}, 2 \mathrm{H}), 1.88(\mathrm{~m}, 1 \mathrm{H}), 1.55(\mathrm{~s}, 9 \mathrm{H}), 1.53(\mathrm{~s}, 9 \mathrm{H}) ;{ }^{13} \mathrm{C} \mathrm{NMR}(100$ $\left.\mathrm{MHz}, \mathrm{CDCl}_{3}\right) \delta 159.4,149.6,149.4,139.7,131.1,123.8,112.9,112.6,85.4,85.3,55.1,53.4$, 53.3, 27.9, 27.0, 24.7; IR (ATR) 2989, 2936, 1732, 1612, 1504, 1352, 1315, 1250, 1144, 1038, 833, $772 \mathrm{~cm}^{-1}$; HRMS (FAB+): $m / z$ calcd. for $\mathrm{C}_{21} \mathrm{H}_{30} \mathrm{~N}_{2} \mathrm{O}_{7} \mathrm{~S}\left([\mathrm{M}]^{+}\right) 454.1774$, found 454.1773. 
<smiles>O=C(O)n1ccc2ccccc21</smiles>

$2 \mathrm{u}$<smiles>O=C(O)c1ccccc1</smiles>

(1.0 equiv)

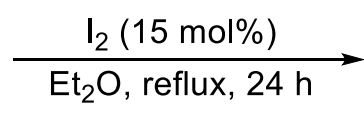<smiles>CC(C)(C)OC(=O)N1c2ccccc2[C@@H]2[C@H]1N(C(=O)OC(C)(C)C)S(=O)(=O)N2C(=O)OCc1ccccc1</smiles>

Following the general procedure (syn-1,2-Diamination), chloramine-BBS (177.4 mg, 0.50 mmol), $N$-Boc-1 $H$-indole (2t: $105.2 \mathrm{mg}, 0.48 \mathrm{mmol})$ and $\mathrm{I}_{2}(19.6 \mathrm{mg}, 0.077 \mathrm{mmol})$ were stirred at reflux temperature in $\mathrm{Et}_{2} \mathrm{O}$ for $24 \mathrm{~h}$. Purification by flash column chromatography on silica gel (hexane/EtOAc $=9: 1)$ gave the product $(5 \mathrm{t}: 120.8 \mathrm{mg}, 49 \%)$ as a white solid. $\mathrm{mp}:$ 77.5$78.3{ }^{\circ} \mathrm{C}$; TLC: $\mathrm{R}_{\mathrm{f}}=0.80$ (hexane/EtOAc $\left.=7: 3\right) ;{ }^{1} \mathrm{H}$ NMR $\left(400 \mathrm{MHz}, \mathrm{CDCl}_{3}\right) \delta 7.67(\mathrm{~d}, J=8.4$ $\mathrm{Hz}, 1 \mathrm{H}), 7.51(\mathrm{~d}, J=7.2 \mathrm{~Hz}, 1 \mathrm{H}), 7.33(\mathrm{dd},=7.28 .4 \mathrm{~Hz}, 1 \mathrm{H}), 7.05(\mathrm{dd}, J=7.2,7.2 \mathrm{~Hz}, 1 \mathrm{H})$, $6.58(\mathrm{~d}, J=8.0 \mathrm{~Hz}, 1 \mathrm{H}), 5.84(\mathrm{~d}, J=8.0 \mathrm{~Hz}, 1 \mathrm{H}), 1.58,(\mathrm{~s}, 9 \mathrm{H}), 1.57(\mathrm{~s}, 9 \mathrm{H}), 1.56(\mathrm{~s}, 9 \mathrm{H}) ;{ }^{13} \mathrm{C}$ $\mathrm{NMR}\left(100 \mathrm{MHz}, \mathrm{CDCl}_{3}\right) \delta 151.4,148.8,148.2,141.4,130.7,126.7,125.8,123.8,116.3,85.9$, 85.7, 83.0, 69.2, 56.8, 28.2, 27.9, 27.8; IR (ATR) 2982, 2934, 1749, 1717, 1481, 1368, 1318, 1252, 1138, 1101, 1049, 839, $752 \mathrm{~cm}^{-1}$; HRMS (FAB+): $\mathrm{m} / z$ calcd. for $\mathrm{C}_{23} \mathrm{H}_{33} \mathrm{~N}_{3} \mathrm{O}_{8} \mathrm{~S}\left([\mathrm{M}]^{+}\right)$ 511.1988 , found 511.1985 .

tri-tert-butyl $\left(3 \mathrm{a} R^{*}, 8 \mathrm{~b} R^{*}\right)$-7-methoxy-3a,8b-dihydro-1H-[1,2,5]thiadiazolo[3,4-b]indole1,3,4-tricarboxylate 2,2-dioxide (2v)<smiles>COc1ccc2c(ccn2C(=O)OCc2ccccc2)c1</smiles>

2v<smiles>O=C(O)c1ccccc1</smiles>

(1.0 equiv)

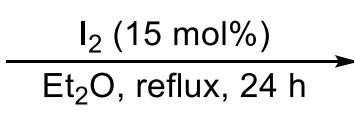<smiles>COc1ccc2c(c1)[C@@H]1[C@@H](N2C(=O)OC(C)(C)C)N(C(=O)OCc2ccccc2)S(=O)(=O)N1C(=O)OCc1ccccc1</smiles>

$5 v$

Following the general procedure (syn-1,2-Diamination), chloramine-BBS (177.9 $\mathrm{mg}, 0.50$ mmol), $N$-Boc-5-methoxy-1H-indole (2t: $127.5 \mathrm{mg}, 0.52 \mathrm{mmol})$ and $\mathrm{I}_{2}(19.2 \mathrm{mg}, 0.076 \mathrm{mmol})$ were stirred at reflux temperature in $\mathrm{Et}_{2} \mathrm{O}$ for $24 \mathrm{~h}$. Purification by flash column chromatography on silica gel (hexane/EtOAc $=9: 1)$ gave the product $(\mathbf{5 t}: 129.1 \mathrm{mg}, 48 \%)$ as white solid. mp: $149.1-149.8{ }^{\circ} \mathrm{C}$; TLC: $\mathrm{R}_{\mathrm{f}}=0.1$ (hexane/EtOAc $\left.=9: 1\right) ;{ }^{1} \mathrm{H}$ NMR $(400 \mathrm{MHz}$, 
$\left.\mathrm{CDCl}_{3}\right) \delta 7.56(\mathrm{~d}, J=8.8 \mathrm{~Hz}, 1 \mathrm{H}), 7.06(\mathrm{~d}, J=2.8 \mathrm{~Hz}, 1 \mathrm{H}), 6.86(\mathrm{dd}, J=2.4,8.8 \mathrm{~Hz}, 1 \mathrm{H}), 6.57$ $(\mathrm{d}, J=8 \mathrm{~Hz}, 1 \mathrm{H}), 5.84(\mathrm{~d}, J=8 \mathrm{~Hz}, 1 \mathrm{H}), 3.76(\mathrm{~s}, 3 \mathrm{H}), 1.59(\mathrm{~s}, 9 \mathrm{H}), 1.56(\mathrm{~s}, 18 \mathrm{H}) ;{ }^{13} \mathrm{C} \mathrm{NMR}$ $\left(100 \mathrm{MHz}, \mathrm{CDCl}_{3}\right) \delta 156.4,151.6,148.9,148.2,134.8,128.2,117.3,116.1,111.1,86.0,85.7$, 82.8, 69.6, 56.8, 55.6, 28.2, 28.0, 27.9; IR (ATR) 2984, 2936, 1761, 1732, 1697, 1489, 1367, 1251, 1142, 851, 793, $773 \mathrm{~cm}^{-1}$; HRMS (FAB+): $m / z$ calcd. for $\mathrm{C}_{24} \mathrm{H}_{35} \mathrm{~N}_{3} \mathrm{O}_{9} \mathrm{~S}\left([\mathrm{M}]^{+}\right) 541.2094$, found 541.2099.

\section{di-tert-butyl $\left(3 R^{*}, 4 R^{*}\right)$-3,4-dipropyl-1,2,5-thiadiazolidine-2,5-dicarboxylate 1,1-dioxide} (5f)

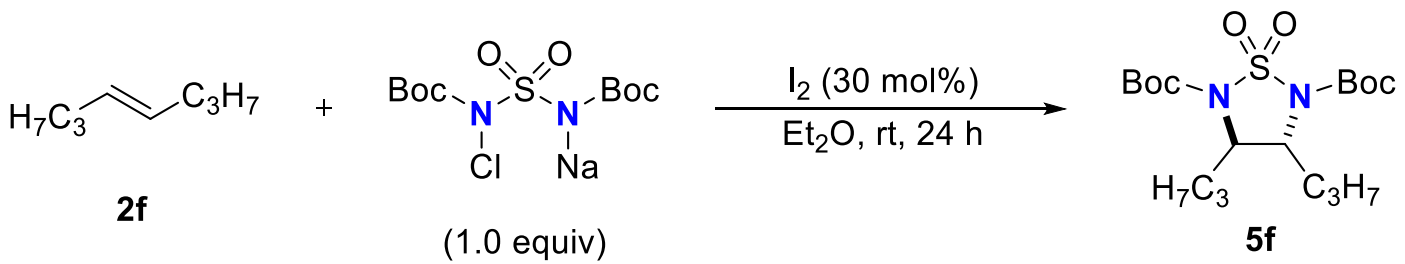

Following the general procedure (syn-1,2-Diamination), chloramine-BBS (177.3 $\mathrm{mg}, 0.50$ mmol), trans-4-octene (2f: $56.9 \mathrm{mg}, 0.51 \mathrm{mmol})$ and $\mathrm{I}_{2}(38.2 \mathrm{mg}, 0.151 \mathrm{mmol})$ were stirred at room temperature in $\mathrm{Et}_{2} \mathrm{O}$ for $24 \mathrm{~h}$. Purification by flash column chromatography on silica gel (hexane/EtOAc $=95: 5)$ gave the product $(\mathbf{5 f}: 115.8 \mathrm{mg}, 57 \%)$ as a colorless oil. TLC: $\mathrm{R}_{\mathrm{f}}=0.33$ (hexane/EtOAc = 9:1); ${ }^{1} \mathrm{H}$ NMR $\left(400 \mathrm{MHz}, \mathrm{CDCl}_{3}\right) \delta 3.93(\mathrm{dd}, J=4.8,8.8 \mathrm{~Hz}, 2 \mathrm{H}), 1.88-$ $1.68(\mathrm{~m}, 4 \mathrm{H}), 1.56(\mathrm{~s}, 18 \mathrm{H}), 1.52-1.25(\mathrm{~m}, 4 \mathrm{H}), 0.97(\mathrm{t}, J=7.2 \mathrm{~Hz}, 6 \mathrm{H}) ;{ }^{13} \mathrm{C} \mathrm{NMR}(100 \mathrm{MHz}$, $\left.\mathrm{CDCl}_{3}\right) \delta 149.1,85.1,57.7,34.7,28.0,18.6,13.6$; IR (ATR) 2961, 2954, 2876, 1788, 1724, 1460, 1356, 1337, 1256, 1182, 1146, 1115, 1049, 845, 824, 764, 752, 737, $712 \mathrm{~cm}^{-1}$; HRMS $(\mathrm{FAB}+): m / z$ calcd. for $\mathrm{C}_{18} \mathrm{H}_{35} \mathrm{~N}_{2} \mathrm{O}_{6} \mathrm{~S}\left([\mathrm{M}+\mathrm{H}]^{+}\right)$407.2216, found 407.2224.

\section{di-tert-butyl $\left(3 R^{*}, 4 S^{*}\right)-3,4$-dipropyl-1,2,5-thiadiazolidine-2,5-dicarboxylate 1,1-dioxide} (5g)

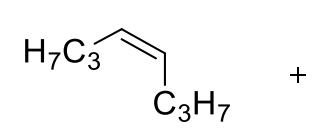

$2 \mathbf{g}$<smiles>CC(C)(C)OC(=O)N([18O])S(=O)(=O)N(Cl)C(=O)O</smiles>

(1.0 equiv)

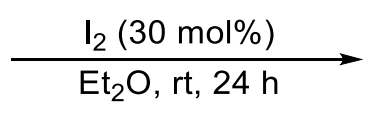<smiles>CCCCC1C([12CH2])N(C(=O)OCc2ccccc2)S(=O)(=O)N1C(=O)O</smiles>

$5 \mathrm{~g}$

Following the general procedure (syn-1,2-Diamination), chloramine-BBS (177.3 $\mathrm{mg}, 0.50$ $\mathrm{mmol})$, cis-4-octene (2g: $55.7 \mathrm{mg}, 0.50 \mathrm{mmol})$ and $\mathrm{I}_{2}(38.1 \mathrm{mg}, 0.150 \mathrm{mmol})$ were stirred at room temperature in $\mathrm{Et}_{2} \mathrm{O}$ for $24 \mathrm{~h}$. Purification by flash column chromatography on silica gel 
(hexane/EtOAc $=95: 5)$ gave the product $(5 \mathrm{~g}: 78.8 \mathrm{mg}, 39 \%)$ as a colorless oil. $\mathrm{TLC}: \mathrm{R}_{\mathrm{f}}=0.60$ (hexane/EtOAc = 7:3); ${ }^{1} \mathrm{H}$ NMR $\left(400 \mathrm{MHz}, \mathrm{CDCl}_{3}\right) \delta 4.21$ (ddd, $\left.J=5.6,5.6,11.2 \mathrm{~Hz}, 2 \mathrm{H}\right)$, 2.19-2.07 (m, 2H), 1.55 (s, 18H), 1.45-1.30 (m, 4H), 0.97 (t, J=7.2 Hz, 6H) (Two C( $\left(\mathrm{sp}^{3}\right)-\mathrm{H}$ signals are overlapped with the ${ }^{t} \mathrm{Bu}$ signal of the 5g.); ${ }^{13} \mathrm{C} \mathrm{NMR}\left(100 \mathrm{MHz}, \mathrm{CDCl}_{3}\right) \delta 149.1$, 85.1, 57.7, 34.7, 28.0, 18.6, 13.6; IR (ATR) 2961, 2954, 2876, 1788, 1724, 1460, 1356, 1337, 1256, 1182, 1146, 1115, 1049, 845, 824, 764, 752, 737, $712 \mathrm{~cm}^{-1}$; HRMS (FAB+): $\mathrm{m} / z$ calcd. for $\mathrm{C}_{18} \mathrm{H}_{35} \mathrm{~N}_{2} \mathrm{O}_{6} \mathrm{~S}\left([\mathrm{M}+\mathrm{H}]^{+}\right)$407.2216, found 407.2221.

\section{di-tert-butyl $\left(3 R^{*}, 4 R^{*}\right)$-3-methyl-4-propyl-1,2,5-thiadiazolidine-2,5-dicarboxylate 1,1- dioxide--benzene $(2 \mathbf{j})$}

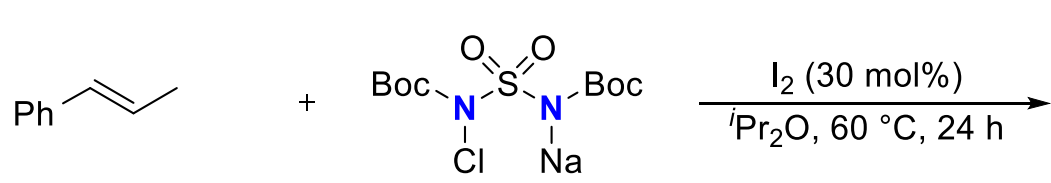

2j

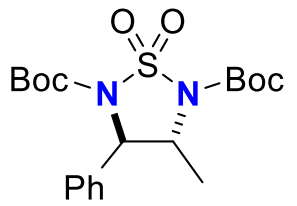

$5 \mathbf{j}$

Following the general procedure (syn-1,2-Diamination), chloramine-BBS (176.4 $\mathrm{mg}, 0.50 \mathrm{mmol})$, trans-6-methylstyrene $(2 \mathrm{~g}: 59.1 \mathrm{mg}, 0.50 \mathrm{mmol})$ and $\mathrm{I}_{2}(37.8 \mathrm{mg}$, $0.149 \mathrm{mmol}$ ) were stirred at $60{ }^{\circ} \mathrm{C}$ in ${ }^{2} \mathrm{Pr}_{2} \mathrm{O}$ for $24 \mathrm{~h}$. Purification by flash column chromatography on silica gel (hexane/EtOAc $=9: 1)$ gave the product (5g: $134.3 \mathrm{mg}$, $65 \%)$ as a white solid. $\mathrm{mp}: 116.9-117.5^{\circ} \mathrm{C} ; \mathrm{R}_{\mathrm{f}} 0.18$ (hexane/EtOAc 9:1); ${ }^{1} \mathrm{H}$ NMR $\left(400 \mathrm{MHz} \mathrm{CDCl}_{3}\right) 7.43-7.32(\mathrm{~m}, 5 \mathrm{H}), 4.84(\mathrm{~s}, 1 \mathrm{H}), 4.19-4.14(\mathrm{~m}, 1 \mathrm{H}), 1.62(\mathrm{~d}, 3 \mathrm{H}$, $J=7.2 \mathrm{~Hz}), 1.55(\mathrm{~s}, 9 \mathrm{H}), 1.47(\mathrm{~s}, 9 \mathrm{H}) ;{ }^{13} \mathrm{C} \mathrm{NMR}\left(100 \mathrm{MHz}, \mathrm{CDCl}_{3}\right)$ 148.8, 148.6, 138.5, 129.0, 128.5, 125.6, 85.55, 85.50, 63.0, 57.3, 28.0, 27.9, 19.4; IR (ATR) 2986, 2972, 2940, 1740, 1732, 1460, 1450, 1395, 1354, 1344, 1281, 1254, 1206, 1171, 1136, 1101, 1084, 1078, 1043, 1026, 982, 926, 910, 843, 833, 820, 810, 781, 760, $737 \mathrm{~cm}^{-}$ 1; MS (FAB-): $\mathrm{m} / z$ (relative intensity, \%) 412 ([M]-, 14), 355 (19), 311 (100); HRMS (FAB-): $m / z$ calcd for $\mathrm{C}_{19} \mathrm{H}_{28} \mathrm{~N}_{2} \mathrm{O}_{6} \mathrm{~S}$ (M) 412.1674, found 412.1670. 


\section{Application}

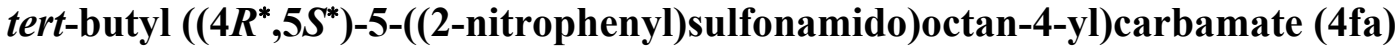

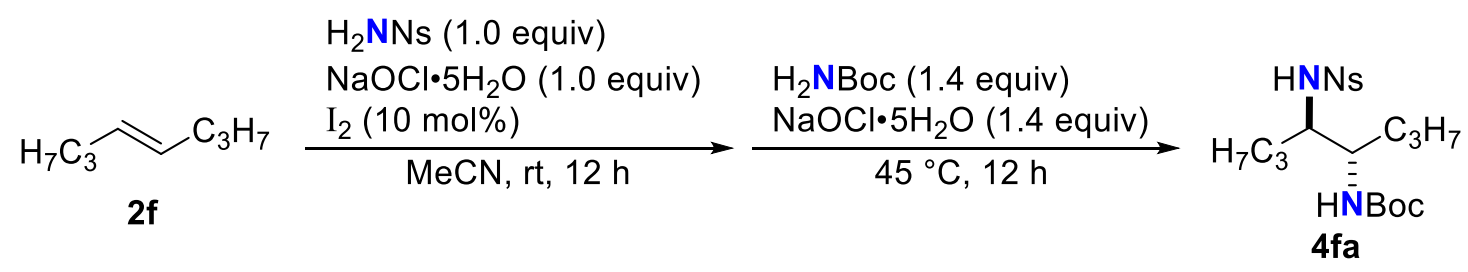

trans-4-octene $(56.2 \mathrm{mg}, 0.50 \mathrm{mmol})$ and sodium hypochlorite pentahydrate $(83.8 \mathrm{mg}, 0.51$ mmol) were added to a stirring solution of 2-nitrobenzenesulfonamide (101.5 mg, $0.50 \mathrm{mmol})$ and iodine (12.9 $\mathrm{mg}, 0.05 \mathrm{mmol})$ at room temperature under a nitrogen atmosphere in $\mathrm{MeCN}$ $(3 \mathrm{~mL})$. The reaction mixture was stirred at room temperature for $12 \mathrm{~h}$ and then tert-butyl carbamate $(82.8 \mathrm{mg}, 0.71 \mathrm{mmol})$ and sodium hypochlorite pentahydrate $(116.1 \mathrm{mg}, 0.71 \mathrm{mmol})$ were added to the above mixture at room temperature. The reaction mixture was warmed to $45^{\circ} \mathrm{C}$ and stirred for $12 \mathrm{~h}$. The reaction was quenched by adding $1 \mathrm{M}$ sodium thiosulfate aqueous solution $(5 \mathrm{~mL})$ and aqueous layer was extracted with EtOAc $(3 \times 30 \mathrm{~mL})$. The combined organic extracts were dried over anhydrous $\mathrm{Na}_{2} \mathrm{SO}_{4}$ and concentrated in vacuo. The crude product was purified by flash column chromatography on silica gel (hexane/EtOAc $=$ 7:3) gave the product (4fa: $182.0 \mathrm{mg}, 85 \%$ ) as a white solid. mp: $130.4-130.8^{\circ} \mathrm{C}$; $\mathrm{TLC}: \mathrm{R}_{\mathrm{f}}=$ 0.35 (hexane/EtOAc = 7:3); ${ }^{1} \mathrm{H}$ NMR $\left(400 \mathrm{MHz}, \mathrm{CDCl}_{3}\right) \delta 8.19-8.08(\mathrm{~m}, 1 \mathrm{H}), 7.92-7.83(\mathrm{~m}$, 1H), 7.78-7.69 (m, 2H), 5.53 (d, $J=9.2 \mathrm{~Hz}, 1 \mathrm{H}), 4.41$ (d, $J=9.2 \mathrm{~Hz}, 1 \mathrm{H}), 3.69-3.57$ (m, 1H), 3.52-3.42 (m, 1H), $1.39(\mathrm{~s}, 9 \mathrm{H}), 1.31-1.10(\mathrm{~m}, 4 \mathrm{H}), 0.98-0.70(\mathrm{~m}, 6 \mathrm{H})\left(\right.$ Four C $\left(\mathrm{sp}^{3}\right)-\mathrm{H}$ signals are overlapped with the ${ }^{t} \mathrm{Bu}$ signal of the 4 fa. $) ;{ }^{13} \mathrm{C} \mathrm{NMR}\left(100 \mathrm{MHz}, \mathrm{CDCl}_{3}\right) \delta 155.7,147.7$, 135.2 , 133.4, 133.0, 130.3, 125.6, 79.5, 58.0, 53.0, 33.2, 32.6, 28.3, 19.3, 19.1, 13.8, 13.7; IR (ATR) 2963, 1682, 1522, 1337, 1302, 1250, 1163, 1055, 854, $743 \mathrm{~cm}^{-1}$; HRMS (FAB-): $\mathrm{m} / \mathrm{z}$ calcd. for $\mathrm{C}_{19} \mathrm{H}_{31} \mathrm{~N}_{3} \mathrm{O}_{6} \mathrm{~S}\left([\mathrm{M}]^{-}\right) 429.1934$, found 429.1939 .

\section{tert-butyl $\left(\left(4 \mathrm{R}^{*}, 5 \mathrm{~S} *\right)-5\right.$-aminooctan-4-yl)carbamate $(4 \mathrm{fb})$}

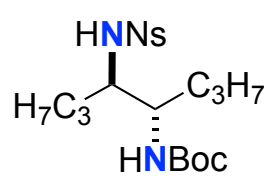

$4 \mathrm{fa}$

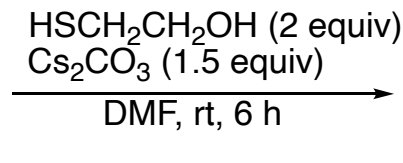

$\frac{\mathrm{HSCH}_{2} \mathrm{CH}_{2} \mathrm{OH} \text { (2 equiv) }}{\mathrm{Cs}_{2} \mathrm{CO}_{3}(1.5 \text { equiv) }}$
$\mathrm{DMF}, \mathrm{rt}, 6 \mathrm{~h}$

2-Mercaptoethanol (46.9 mg, $0.6 \mathrm{mmol}$ ) was added to a stirred solution of $\mathbf{4 f a}$ (128.7 $\mathrm{mg}, 0.3$ mmol), cesium carbonate (146.1 $\mathrm{mg}, 0.45 \mathrm{mmol})$ at room temperature under a nitrogen atmosphere in DMF $(1.5 \mathrm{~mL})$. The reaction mixture was stirred for at room temperature for 6 
h. After stirring, aqueous $\mathrm{NaOH}(2 \mathrm{M}, 5 \mathrm{~mL})$ was added and the aqueous layer was extracted with $\mathrm{CH}_{2} \mathrm{Cl}_{2}(3 \times 30 \mathrm{~mL})$. The combined organic extracts were dried over anhydrous $\mathrm{Na}_{2} \mathrm{SO}_{4}$ and concentrated in vacuo. The crude product was purified by flash column chromatography on silica gel $\left(\mathrm{CHCl}_{3} / \mathrm{MeOH}\right)$ gave the product (4fb: $\left.46.2 \mathrm{mg}, 63 \%\right)$ as a colorless liquid. TLC: $\mathrm{R}_{\mathrm{f}} 0.08\left(\mathrm{CHCl}_{3} / \mathrm{MeOH}=95: 5\right) ;{ }^{1} \mathrm{H}$ NMR $\left(400 \mathrm{MHz}, \mathrm{CDCl}_{3}\right) \delta 4.69$ (br s, $\left.1 \mathrm{H}\right), 3.54$ (br s, $\left.1 \mathrm{H}\right)$, 2.75 (br s, 1H), 1.52-1.18 (m, 19H), 0.93 (t, 6H, $\left.J=7.2 \mathrm{~Hz}) ;{ }^{13} \mathrm{C} \mathrm{NMR} \mathrm{(100} \mathrm{MHz,} \mathrm{CDCl}_{3}\right) \delta$ 156.1, 78.9, 54.9, 54.7, 36.5, 31.9, 28.4, 19.8, 19.5, 14.14, 14.07; MS (FAB+): $m / z$ (relative intensity, \%) $245\left([\mathrm{M}+\mathrm{H}]^{+}, 98\right), 186(100), 172$ (7); HRMS (FAB+): $m / z$ calcd for $\mathrm{C}_{13} \mathrm{H}_{29} \mathrm{~N}_{2} \mathrm{O}_{2}$ $(\mathrm{M}+\mathrm{H})$ 245.2224, found 245.2232.

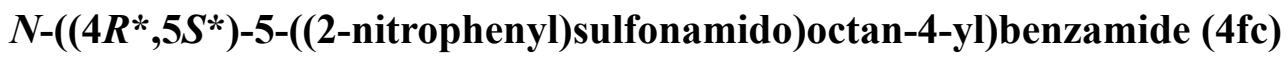
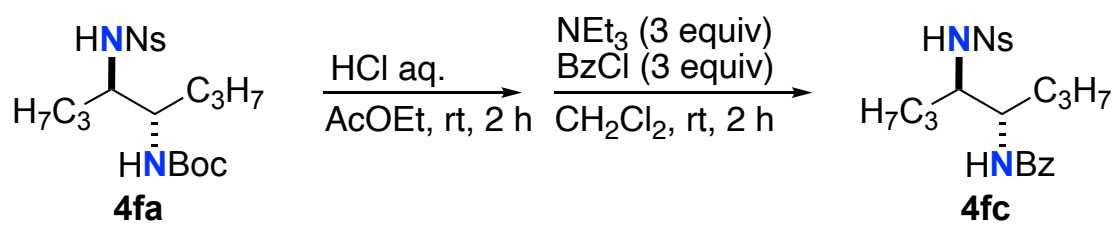

Concentrated aqueous $\mathrm{HCl}(528 \mu \mathrm{L})$ was added to a stirred solution of $\mathbf{4 f a}(128.7 \mathrm{mg}, 0.3$ $\mathrm{mmol})$ in ethyl acetate $(3.0 \mathrm{~mL})$ at room temperature and stirred for $2 \mathrm{~h}$. After stirring, the solvent was removed under vacuo, and $\mathrm{CH}_{2} \mathrm{Cl}_{2}(3 \mathrm{~mL})$ was added followed by cooled to $0{ }^{\circ} \mathrm{C}$. Triethyamine ( $91.1 \mathrm{mg}, 0.9 \mathrm{mmol}$ ) and benzoyl chloride (126.5 mg, $0.9 \mathrm{mmol}$ ) were added to the cooled solution. The mixture was warmed to room temperature and stirred for $2 \mathrm{~h}$. After stirring, water $(10 \mathrm{~mL})$ was added and the aqueous layer was extracted with EtOAc $(3 \times 30$ $\mathrm{mL}$ ). The combined organic extracts were dried over anhydrous $\mathrm{Na}_{2} \mathrm{SO}_{4}$ and concentrated in vacuo. The crude product was purified by flash column chromatography on silica gel (hexane/EtOAc = 7:3) gave the product (4fc: $125.6 \mathrm{mg}, 97 \%)$ as a white solid.; ${ }^{1} \mathrm{H}$ NMR (400 $\left.\mathrm{MHz}, \mathrm{CDCl}_{3}\right) \delta 8.05-8.03(\mathrm{~m}, 1 \mathrm{H}), 7.77-7.75(\mathrm{~m}, 3 \mathrm{H}), 7.61(\mathrm{t}, 2 \mathrm{H}, J=4.0 \mathrm{~Hz}), 7.45(\mathrm{t}, 1 \mathrm{H}, J$ $=7.6 \mathrm{~Hz}), 6.39$ (br d, 1H, $J=8.8 \mathrm{~Hz}), 5.34$ (br d, $1 \mathrm{H}, J=8.8 \mathrm{~Hz}), 4.20-4.15(\mathrm{~m}, 1 \mathrm{H}), 3.68-$ $3.64(\mathrm{~m}, 1 \mathrm{H}), 1.58-1.10(\mathrm{~m}, 10 \mathrm{H}), 0.94(\mathrm{t}, 3 \mathrm{H}, J=7.2 \mathrm{~Hz}), 0.78(\mathrm{t}, 3 \mathrm{H}, J=7.2 \mathrm{~Hz}) ;{ }^{13} \mathrm{C} \mathrm{NMR}$ $\left(100 \mathrm{MHz}, \mathrm{CDCl}_{3}\right) \delta 167.2,147.7,134.2,134.1,133.5,132.8,131.5,130.7,128.6,127.0,125.2$, 58.6, 51.8, 35.1, 31.0, 19.4, 19.2, 13.9, 13.6; IR (ATR) v 3319, 2959, 2930, 2870, 2862, 1634, 1605,1582 , 1541, 1489, 1435, 1360, 1335, 1302, 1275, 1244, 1163, 1121, 1076, 1059, 1036, $1020,926,854,835,804,783,744,729,700 \mathrm{~cm}^{-1}$; MS (FAB+): $m / z$ (relative intensity, \%) 434 $\left([\mathrm{M}+\mathrm{H}]^{+}, 61\right), 307$ (18), 289 (10), 176 (15), 154 (100), ; HRMS (FAB+): m/z calcd for $\mathrm{C}_{21} \mathrm{H}_{28} \mathrm{~N}_{3} \mathrm{O}_{5} \mathrm{~S}(\mathrm{M}+\mathrm{H})$ 434.1744, found 434.1748. 


\section{$N, N^{\prime}$-(1-phenylethane-1,2-diyl)bis( $N, N^{\prime}$-dimethyl-2-nitrobenzenesulfonamide) (3ka)}

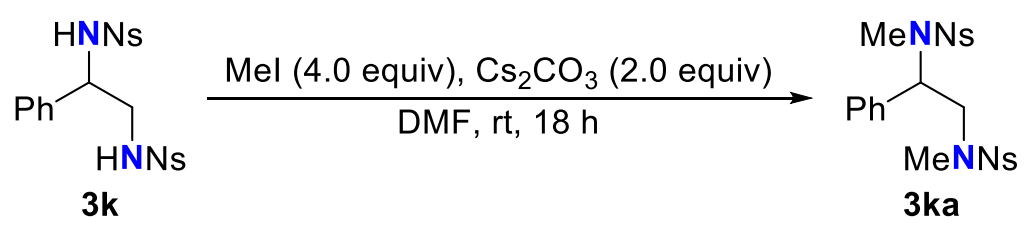

Iodomethane $(282.2 \mathrm{mg}, 1.99 \mathrm{mmol})$ was added to a stirring solution of $N, N$ '-(1-phenylethane1,2-diyl)bis(2-nitrobenzenesulfonamide) $(3 \mathrm{k}: 253.6 \mathrm{mg}, 0.50 \mathrm{mmol})$ and cesium carbonate (327.1 $\mathrm{mg}, 1.00 \mathrm{mmol})$ at room temperature under a nitrogen atmosphere in DMF $(5 \mathrm{~mL})$. The reaction mixture was stirred at room temperature for $18 \mathrm{~h}$. After stirring, the reaction mixture was diluted by water and extracted by $\mathrm{Et}_{2} \mathrm{O}(3 \times 40 \mathrm{~mL})$. The combined organic extracts were washed by water $(3 \times 30 \mathrm{~mL})$ and dried over anhydrous $\mathrm{Na}_{2} \mathrm{SO}_{4}$, then concentrated in vacuo. Purification of the crude product by flash column chromatography (hexane/EtOAc $=7: 3$ ) on silica gel gave the product (3ka: $256.2 \mathrm{mg}, 96 \%$ ) as a white solid. mp: $78.5-79.3{ }^{\circ} \mathrm{C} ;{ }^{1} \mathrm{H}$ NMR $\left(400 \mathrm{MHz}, \mathrm{CDCl}_{3}\right) \delta 8.03-7.88(\mathrm{~m}, 2 \mathrm{H}), 7.80-7.56(\mathrm{~m}, 6 \mathrm{H}), 7.40-7.21(\mathrm{~m}, 5 \mathrm{H}), 5.45(\mathrm{dd}, J=$ 6.0, 9.2 Hz, 1H), 4.02 (dd, $J=9.2,14.0 \mathrm{~Hz}, 1 \mathrm{H}), 3.56(\mathrm{dd}, J=6.0,14.0 \mathrm{~Hz}, 1 \mathrm{H}), 2.87$ (s, 3H), $2.84(\mathrm{~s}, 3 \mathrm{H}) ;{ }^{13} \mathrm{C} \mathrm{NMR}\left(100 \mathrm{MHz}, \mathrm{CDCl}_{3}\right) \delta 148.4,148.1,134.4,133.9,133.5,133.1,132.0$, 131.7, 131.0, 130.8, 130.4, 128.8, 128.4, 124.1, 124.0, 57.3, 49.8, 34.8, 29.9 (Two sp ${ }^{2}$ signals were not observed because of overlapping.); IR (ATR) 1539, 1439, 1348, 1163, 1125, 1059, 989, 953, 853, $758 \mathrm{~cm}^{-1}$; HRMS (FAB+): $m / z$ calcd. for $\mathrm{C}_{22} \mathrm{H}_{23} \mathrm{~N}_{4} \mathrm{O}_{8} \mathrm{~S}_{2}\left([\mathrm{M}+\mathrm{H}]^{+}\right)$535.0957, found 535.0959 .

di-tert-butyl $\left(3 \mathrm{a} R^{*}, 6 \mathrm{a} S^{*}\right)$-cyclopenta[c][1,2,5]thiadiazolidine-1,3-dicarboxylate 2,2dioxide (5a)

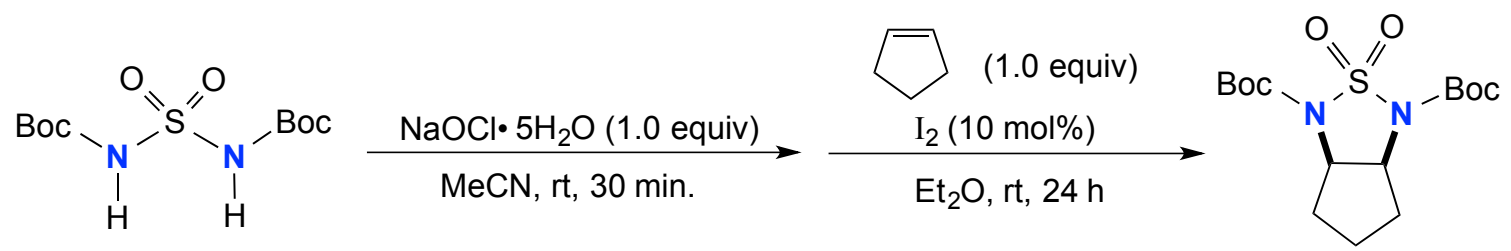

$5 \mathbf{a}$

(one-pot process)

Following the general procedure (syn-1,2-Diamination), A solution of BBS (148.3 mg, 0.5 $\mathrm{mmol})$ and sodium hypochlorite pentahydrate $(82.5 \mathrm{mg}, 0.5 \mathrm{mmol})$ under a nitrogen atmosphere in $\mathrm{MeCN}(3 \mathrm{~mL})$ was stirred at room temperature for $0.5 \mathrm{~h}$. After stirring, $\mathrm{MeCN}$ was reduced under vacuo, and $\mathrm{Et}_{2} \mathrm{O}(3 \mathrm{~mL})$, iodine $(12.7 \mathrm{mg}, 0.05 \mathrm{mmol})$ and cyclopentene (2a: $34.6 \mathrm{mg}, 0.51 \mathrm{mmol}$ ) were added to the residue and stirred at room temperature for $24 \mathrm{~h}$. 
Purification by flash column chromatography on silica gel (hexane/EtOAc $=9: 1$ ) gave the product (5a: $102.7 \mathrm{mg}, 57 \%$ ) as a white solid.

\section{$\left(1 R^{*}, 2 R^{*}\right)-2,3-d i h y d r o-1 H$-indene-1,2-diaminium ditosylate (4ea)}

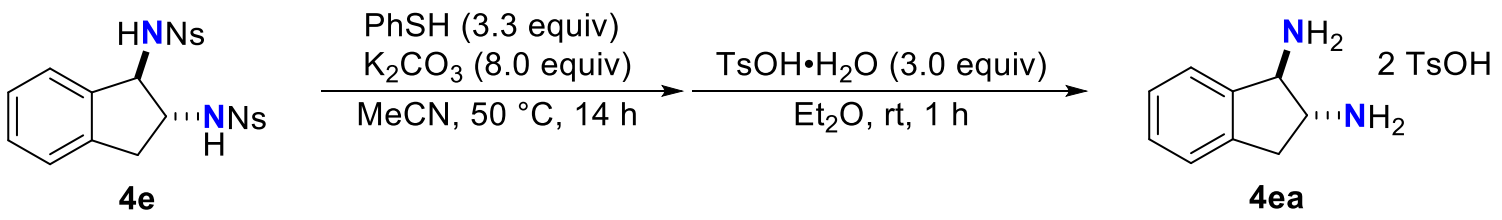

Benzenethiol $(195.9 \mathrm{mg}, 0.99 \mathrm{mmol})$ was added to a stirring solution of trans- $N, N^{\prime}-(2,3-$ dihydro-1H-indene-1,2-diyl)bis(2-nitrobenzenesulfonamide) (4e: $155.8 \mathrm{mg}, 0.30 \mathrm{mmol})$ and potassium carbonate $(331.4 \mathrm{mg}, 2.40 \mathrm{mmol})$ at room temperature under a nitrogen atmosphere in $\mathrm{MeCN}(5 \mathrm{~mL})$. The reaction mixture was stirred at $50{ }^{\circ} \mathrm{C}$ for $14 \mathrm{~h}$. After stirring, the white precipitate was removed by filtration, washed with $\mathrm{Et}_{2} \mathrm{O}$. The filtrate was concentrated in vacuo. The residue was dissolved in $\mathrm{Et}_{2} \mathrm{O}(5 \mathrm{~mL})$. This solution was added drop-wise to a solution of p-toluenesulfonate monohydrate $(172.5 \mathrm{mg}, 0.91 \mathrm{mmol})$ in $\mathrm{Et}_{2} \mathrm{O}(3 \mathrm{~mL})$. The reaction was kept stirring at room temperature for $1 \mathrm{~h}$. After the reaction mixture was cooled to $0{ }^{\circ} \mathrm{C}$, washing the precipitate with $\mathrm{Et}_{2} \mathrm{O}$ and $\mathrm{CH}_{2} \mathrm{Cl}_{2}$ gave the product (4ea: $108.2 \mathrm{mg}, 73 \%$ ) as a white solid. ${ }^{1} \mathrm{H}$ NMR (400 MHz, DMSO- $\left.d_{6}\right) \delta 8.61$ (brs, 3H), 8.38 (brs, $3 \mathrm{H}$ ), 7.60 (d, $\left.J=7.2 \mathrm{~Hz}, 1 \mathrm{H}\right), 7.51$ (d, $J=7.8 \mathrm{~Hz}, 4 \mathrm{H}), 7.44-7.32$ (m, 3H), 7.14 (d, $J=7.8 \mathrm{~Hz}, 4 \mathrm{H}), 4.92-4.86$ (m, 1H), 4.09-3.99 (m, 1H), $3.48(\mathrm{dd}, J=8.6,17.0 \mathrm{~Hz}, 1 \mathrm{H}), 3.03(\mathrm{dd}, J=6.2,17.0 \mathrm{~Hz}, 1 \mathrm{H}), 2.30(\mathrm{~s}, 6 \mathrm{H}) ;{ }^{13} \mathrm{C}$ NMR (100 MHz, DMSO- $\left.d_{6}\right) \delta 144.9,140.0,138.2,136.0,129.9,128.2,127.7,125.5,125.1$, $125.0,58.4,54.4,35.4,20.8$. The spectral data for this compound were in good agreement with the reported data. ${ }^{2}$

$\left(1 R^{*}, 2 S^{*}\right)-2,3-d i h y d r o-1 H$-indene-1,2-diaminium ditosylate (5ea)

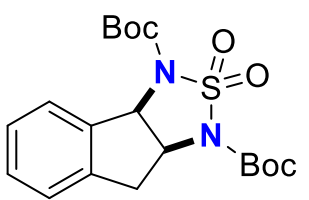

$5 e$

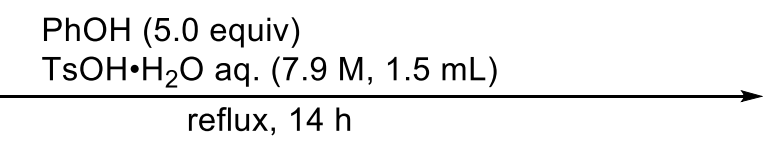

reflux, $14 \mathrm{~h}$

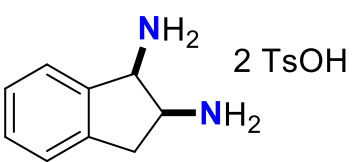

5ea

7.9 $\mathrm{M} p$-toluenesulfonate aqueous solution $(1.5 \mathrm{~mL})$ was added to di-tert-butyl $\left(3 \mathrm{a} R^{*}, 8 \mathrm{~b} S^{*}\right)$ inda[1,2-c][1,2,5]thiadiazolidine-1,3-dicarboxylate 2,2-dioxide (5a: $103.0 \mathrm{mg}, 0.25 \mathrm{mmol}$ ) and phenol (118.8 mg, $1.26 \mathrm{mmol})$ in two-neck glass tubes under a nitrogen atmosphere. The reaction mixture was heated to reflux for $14 \mathrm{~h}$. Upon completion, the reaction mixture was concentrated in vacuo. Washing the residue gave the product (5aa: $95.0 \mathrm{mg}, 77 \%$ ) as a white 
solid. ${ }^{1} \mathrm{H}$ NMR (400 MHz, DMSO- $\left.d_{6}\right) \delta 8.55$ (brs, 3H), 8.44 (brs, $\left.3 \mathrm{H}\right), 7.60$ (d, $\left.J=7.2 \mathrm{~Hz}, 1 \mathrm{H}\right)$, $7.51(\mathrm{~d}, J=7.8 \mathrm{~Hz}, 4 \mathrm{H}), 7.44-7.30(\mathrm{~m}, 3 \mathrm{H}), 7.14(\mathrm{~d}, J=7.8 \mathrm{~Hz}, 1 \mathrm{H}), 4.92-4.86(\mathrm{~m}, 1 \mathrm{H}), 4.24$ $4.12(\mathrm{~m}, 1 \mathrm{H}), 3.28(\mathrm{dd}, J=7.6,16.4 \mathrm{~Hz}, 1 \mathrm{H}), 3.21(\mathrm{dd}, J=8.0,16.4 \mathrm{~Hz}, 1 \mathrm{H}), 2.30(\mathrm{~s}, 6 \mathrm{H}) ;{ }^{13} \mathrm{C}$ NMR (100 MHz, DMSO-d6) $\delta 144.5,140.0,138.6,136.0,130.1,128.4,127.6,125.9,125.6$, $125.3,54.8,51.9,34.1,20.9$. The spectral data for this compound was were in good agreement with the reported data. ${ }^{2}$

\section{Starting Materials}

Alkenes $\mathbf{1} \mathbf{j}^{3}, \mathbf{1} \mathbf{p}^{4}, \mathbf{2} \mathbf{n}^{5}, \mathbf{2} \mathbf{r}^{6}, \mathbf{2} \mathbf{t}^{7}, \mathbf{2} \mathbf{u}^{8}$ and $\mathbf{2} \mathbf{v}^{9}$ were prepared by known methods with a modification when necessary. Analytical data for $\mathbf{1 j}^{3}, \mathbf{1} \mathbf{p}^{4}, \mathbf{2} \mathbf{n}^{5}, \mathbf{2} \mathbf{r}^{6}, \mathbf{2} \mathbf{t}^{7}, \mathbf{2} \mathbf{u}^{8}$ and $\mathbf{2} \mathbf{v}^{9}$ were in good agreement with reported data. $N, N^{\prime}$-Bis(tert-butoxycarbonyl)sulfamide (BBS) was prepared by new method. Other alkenes and reagents were purchased and used as obtained. The synthetic methods and analytical data for unreported products in supplementary text were also shown in this section.

\section{$\mathrm{N}$-allyl-4-methyl-benzenesulfonamide (1j)}

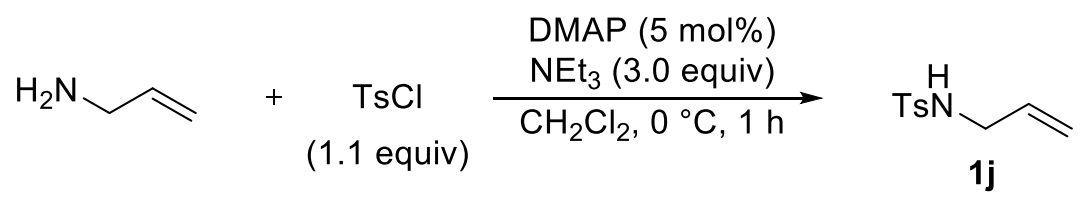

The compound was prepared by the reported procedure ${ }^{3}$ with a slight modification. ${ }^{1} \mathrm{H}$ NMR $\left(400 \mathrm{MHz}, \mathrm{CDCl}_{3}\right) \delta 7.76(\mathrm{~d}, J=8.4 \mathrm{~Hz}, 2 \mathrm{H}), 7.32(\mathrm{~d}, J=8.4 \mathrm{~Hz}, 2 \mathrm{H}), 5.79-5.68(\mathrm{~m}, 1 \mathrm{H}), 5.17$ $(\mathrm{d}, J=17.2 \mathrm{~Hz}, 1 \mathrm{H}), 5.11(\mathrm{~d}, J=10.2 \mathrm{~Hz}, 1 \mathrm{H}), 4.46-4.33(\mathrm{~m}, 1 \mathrm{H}), 3.65-3.55(\mathrm{~m}, 2 \mathrm{H}), 2.44$ (s, $3 \mathrm{H}) ;{ }^{13} \mathrm{C} \mathrm{NMR}\left(100 \mathrm{MHz}, \mathrm{CDCl}_{3}\right) \delta 143.5,136.9,132.9,129.7,127.1,117.7,45.7,21.5$; IR (ATR) 3244, 2930, 2862, 1595, 1423, 1317, 1157, 1063, 935, 876, $810 \mathrm{~cm}^{-1}$; HRMS (EI+): $m / z$ calcd. for $\mathrm{C}_{10} \mathrm{H}_{13} \mathrm{NO}_{2} \mathrm{~S}\left(\mathrm{M}^{+}\right) 211.0667$, found 211.0665 .

\section{3-vinylanisole (1p)}<smiles>COc1cccc(C=O)c1</smiles><smiles></smiles>

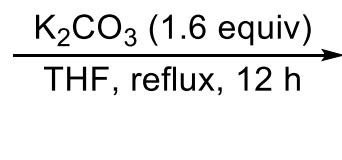<smiles>C=Cc1cccc(OC)c1</smiles> 
The compound was prepared by the reported procedure ${ }^{4}$ with a slight modification. ${ }^{1} \mathrm{H}$ NMR $\left(400 \mathrm{MHz}, \mathrm{CDCl}_{3}\right) \delta 7.30-7.18(\mathrm{~m}, 1 \mathrm{H}), 7.01(\mathrm{~d}, J=7.6 \mathrm{~Hz}, 1 \mathrm{H}), 6.98-6.92(\mathrm{~m}, 1 \mathrm{H}), 6.85-$ $6.78(\mathrm{~m}, 1 \mathrm{H}), 6.68(\mathrm{dd}, J=10.8,15.6 \mathrm{~Hz}, 1 \mathrm{H}), 5.74(\mathrm{~d}, J=15.6 \mathrm{~Hz}, 1 \mathrm{H}), 5.25(\mathrm{~d}, J=10.8 \mathrm{~Hz}$, 1H), $3.82(\mathrm{~s}, 3 \mathrm{H}) ;{ }^{13} \mathrm{C}$ NMR $\left(100 \mathrm{MHz}, \mathrm{CDCl}_{3}\right) \delta 159.8,139.0,136.7,129.4,118.8,114.1$, 113.4, 111.5, 55.1; IR (ATR) 3003, 2833, 1576, 1487, 1287, 1261, 1153, 1034, 989, 907, 783, $716 \mathrm{~cm}^{-1}$; HRMS (EI+): $\mathrm{m} / z$ calcd. for $\mathrm{C}_{9} \mathrm{H}_{10} \mathrm{O}\left(\mathrm{M}^{+}\right)$134.0732, found 134.0734.

\section{cis-(hex-3-en-1-yloxy)tert-butyldimethylsilane (2n)}

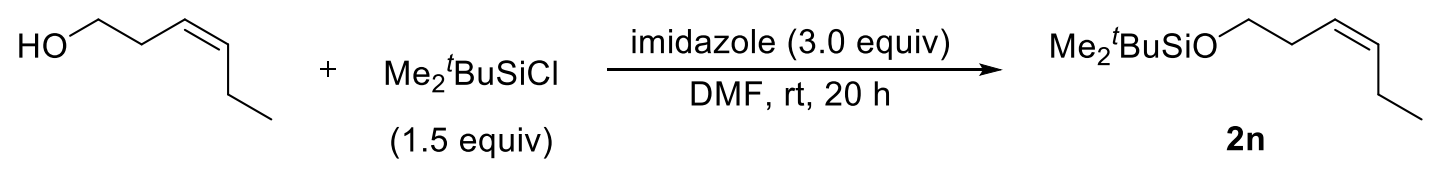

The compound was prepared by the reported procedure ${ }^{5}$ with a slight modification. ${ }^{1} \mathrm{H}$ NMR (400 MHz, $\left.\mathrm{CDCl}_{3}\right) \delta 5.51-5.42(\mathrm{~m}, 1 \mathrm{H}), 5.40-5.28(\mathrm{~m}, 1 \mathrm{H}), 3.60(\mathrm{t}, J=6.8 \mathrm{~Hz}, 2 \mathrm{H}), 2.27$ (q, $J=7.2 \mathrm{~Hz}, 2 \mathrm{H}), 2.06(\mathrm{dt}, J=6.8,6.8 \mathrm{~Hz}, 2 \mathrm{H}), 0.96(\mathrm{t}, J=7.2 \mathrm{~Hz}, 3 \mathrm{H}), 0.90$ (s, 9H), 0.06 (s, $6 \mathrm{H}) ;{ }^{13} \mathrm{C}$ NMR $\left(100 \mathrm{MHz}, \mathrm{CDCl}_{3}\right) \delta 133.5,125.0,63.0,31.0,25.9,20.6,18.4,14.3,-5.29$; IR (ATR) 2957, 2930, 2859, 1738, 1472, 1362, 1098, 937, 835, $773 \mathrm{~cm}^{-1}$; HRMS (CI+): $m / z$ calcd. for $\mathrm{C}_{12} \mathrm{H}_{27} \mathrm{OSi}\left([\mathrm{M}+\mathrm{H}]^{+}\right)$215.1831, found 215.1832.

\section{6-bromo-1H-indene (2r)}
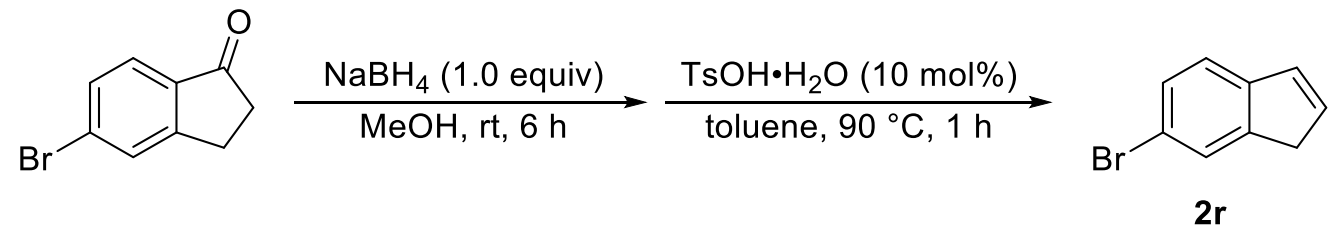

The compound was prepared by the reported procedure ${ }^{6}$ with a slight modification. ${ }^{1} \mathrm{H}$ NMR $\left(400 \mathrm{MHz}, \mathrm{CDCl}_{3}\right) \delta 7.61-7.56(\mathrm{~m}, 1 \mathrm{H}), 7.42-7.36(\mathrm{~m}, 1 \mathrm{H}), 7.27-7.20(\mathrm{~m}, 1 \mathrm{H}), 6.84-6.77$ (m, $1 \mathrm{H}), 6.56-6.48(\mathrm{~m}, 1 \mathrm{H}), 3.41-3.31(\mathrm{~m}, 2 \mathrm{H}) ;{ }^{13} \mathrm{C} \mathrm{NMR}\left(100 \mathrm{MHz}, \mathrm{CDCl}_{3}\right) \delta 145.7,143.6,134.5$, 131.4, 129.2, 126.9, 122.0, 118.6, 38.9; IR (ATR) 2978, 1732, 1450, 1379, 1329, 1250, 1155 , 1117, 1078, 1022, 853, 766, $745 \mathrm{~cm}^{-1}$; HRMS (EI+): $m / z$ calcd. for $\mathrm{C}_{9} \mathrm{H}_{7} \mathrm{Br}\left(\mathrm{M}^{+}\right)$193.9731, found 193.9728 . 


\section{7-methoxy-1.2-dihydronaphthalene (2t)}

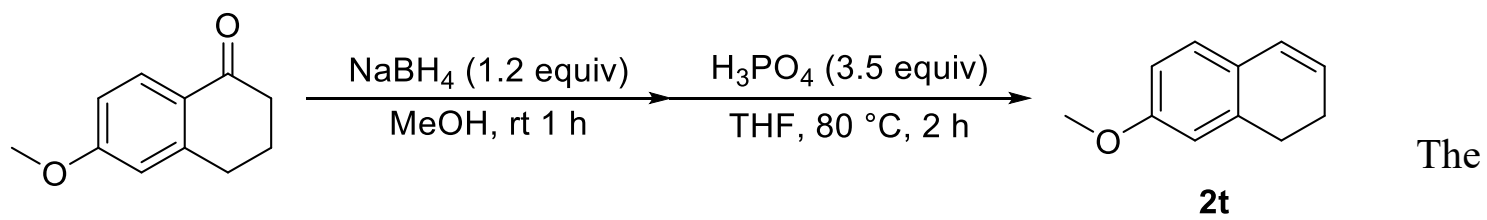

compound was prepared by the reported procedure. ${ }^{71} \mathrm{H}$ NMR $\left(400 \mathrm{MHz}, \mathrm{CDCl}_{3}\right) \delta 6.95(\mathrm{~d}, J$ $=9.2 \mathrm{~Hz}, 1 \mathrm{H}), 6.70-6.67(\mathrm{~m}, 2 \mathrm{H}), 6.41(\mathrm{~d}, \mathrm{~J}=9.2 \mathrm{~Hz}, 1 \mathrm{H}), 5.92-5.87(\mathrm{~m}, 1 \mathrm{H}), 3.80(\mathrm{~s}, 3 \mathrm{H})$, $2.77(\mathrm{t}, 2 \mathrm{H}), 2.32-2.26(\mathrm{~m}, 2 \mathrm{H}) ;{ }^{13} \mathrm{C} \mathrm{NMR}\left(100 \mathrm{MHz}, \mathrm{CDCl}_{3}\right) \delta 158.4,136.9,127.1,127.0$, 126.7, 125.6, 113.6, 110.8, 54.8, 27.8, 22.8; IR (ATR) 3030, 2934, 2830, 1607, 1570, 143030, 2934, 2830, 1607, 1570, 1499, 1427, 1306, 1277, 1250, 1150, 1123, 1038, $816 \mathrm{~cm}^{-1}$; HRMS (EI+): $m / z$ calcd. for $\mathrm{C}_{11} \mathrm{H}_{12} \mathrm{O}\left(\mathrm{M}^{+}\right) 160.0888$, found 160.0889 .

\section{tert-butyl-1H-indole-1-carboxylate (2u)}

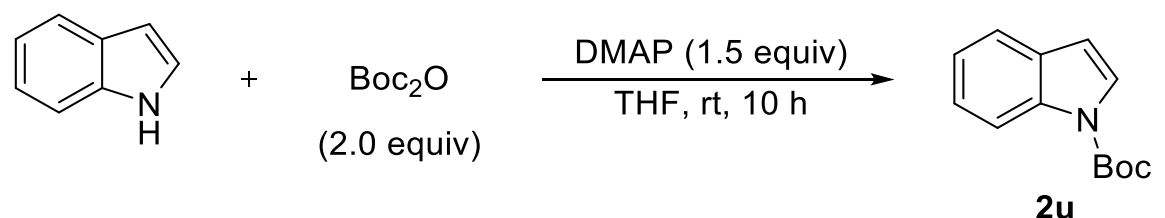

The compound was prepared by the reported procedure. ${ }^{8}{ }^{1} \mathrm{H}$ NMR $\left(400 \mathrm{MHz}, \mathrm{CDCl}_{3}\right) \delta 8.15$ $(\mathrm{d}, J=7.2 \mathrm{~Hz}, 1 \mathrm{H}), 7.65-7.52(\mathrm{~m}, 2 \mathrm{H}), 7.39-7.13(\mathrm{~m}, 2 \mathrm{H}), 6.56(\mathrm{~d}, J=3.6 \mathrm{~Hz}, 1 \mathrm{H}), 1.66(\mathrm{~s}$, 9H); ${ }^{13} \mathrm{C} \mathrm{NMR}\left(100 \mathrm{MHz}, \mathrm{CDCl}_{3}\right) \delta 149.8,135.1,130.5,125.8,124.1,122.6,120.9,115.1$, 107.2, 83.6, 28.1; IR (ATR) 2978, 1732, 1450, 1329, 1250, 1121, 1155, 1117, 1078, 1022, 853, $745 \mathrm{~cm}^{-1}$; HRMS (EI+): $m / z$ calcd. for $\mathrm{C}_{13} \mathrm{H}_{15} \mathrm{NO}_{2}\left(\mathrm{M}^{+}\right) 217.1103$, found 217.1101 .

tert-butyl 5-methoxy-1H-indole-1-carboxylate (2v)

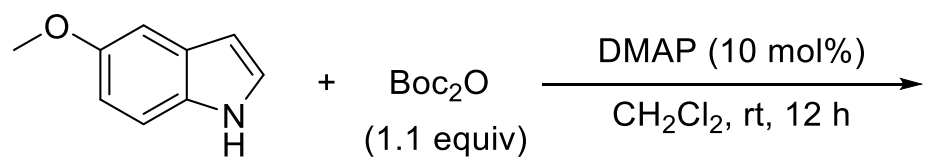<smiles>COc1ccc2c(ccn2C(=O)OCc2ccccc2)c1</smiles>

2v

The compound was prepared by the reported procedure. ${ }^{9} \mathrm{H}$ NMR $\left(400 \mathrm{MHz}, \mathrm{CDCl}_{3}\right) \delta 8.01$ (s, 1H), $7.56(\mathrm{~s}, 1 \mathrm{H}), 7.02(\mathrm{~d}, \mathrm{~J}=2.4 \mathrm{~Hz}, 1 \mathrm{H}), 6.92(\mathrm{dd}, \mathrm{J}=2.8,8.8 \mathrm{~Hz}, 1 \mathrm{H}), 6.49(\mathrm{~d}, \mathrm{~J}=4.0$ $\mathrm{Hz}, 1 \mathrm{H}), 3.84$ (s, 3H), 1.658 (s, 9H); ${ }^{13} \mathrm{C} \mathrm{NMR}\left(100 \mathrm{MHz}, \mathrm{CDCl}_{3}\right) \delta 155.7,149.7,131.3,129.8$, 126.4, 115.7, 112.9, 107.0, 103.3, 83.4, 55.5, 28.1; IR (ATR) 2939, 1724, 1474, 1377, 1271, 1258, 1161, 1134, 1088, 1024, 851, 795, 758, $723 \mathrm{~cm}^{-1}$; HRMS (EI+): $\mathrm{m} / z$ calcd. for $\mathrm{C}_{14} \mathrm{H}_{17} \mathrm{NO}_{3}\left(\mathrm{M}^{+}\right) 247.1208$, found 247.1213. 


\section{$N$-chloro- $N$-sodio-2-nitrobenzenesulfonamide dihydrate}

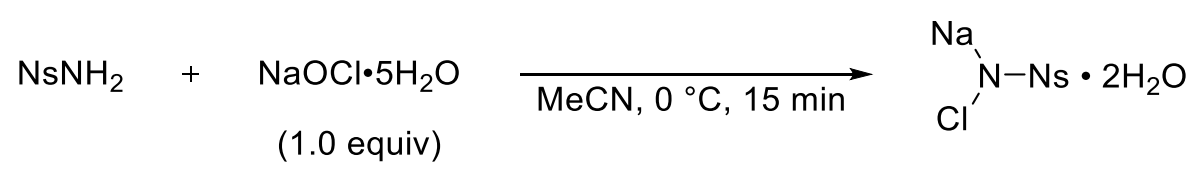

Sodium hypochlorite pentahydrate $(165.4 \mathrm{mg}, 1.01 \mathrm{mmol})$ was added to a stirring solution of 2-nitrobenzenesulfonamide $(201.1 \mathrm{mg}, 0.99 \mathrm{mmol})$ at $0{ }^{\circ} \mathrm{C}$ under a nitrogen atmosphere in $\mathrm{MeCN}(3 \mathrm{~mL})$. The reaction mixture was stirred at $0{ }^{\circ} \mathrm{C}$ for $15 \mathrm{~min}$. After stirring, the reaction mixture was concentrated in vacuo. The residual solid was diluted by water $(30 \mathrm{~mL})$ and $\mathrm{Et}_{2} \mathrm{O}$ $(40 \mathrm{~mL})$ and washed by $\mathrm{Et}_{2} \mathrm{O}(2 \times 40 \mathrm{~mL})$. The concentration of the aqueous layer in vacuo gave the pure product (91\%). Yield is determined using 1,1,2,2-tetrachloroethane as the internal standard by ${ }^{1} \mathrm{H}$ NMR. mp: $93.8-94.0{ }^{\circ} \mathrm{C} ;{ }^{1} \mathrm{H}$ NMR (400 MHz, DMSO- $d_{6}$ ) $\delta 7.92-7.80$ (m, 1H), 7.71-7.51 (m, $3 \mathrm{H}) ;{ }^{13} \mathrm{C}$ NMR (100 MHz, DMSO- $\left.d_{6}\right) \delta 149.0,136.4,131.5,131.1$, 130.6, 123.3; IR (ATR) 3526, 1541, 1393, 1356, 1273, 1142, 1115, 1057, 939, 770, $727 \mathrm{~cm}^{-1}$; HRMS (FAB+): $m / z$ calcd. for $\mathrm{C}_{6} \mathrm{H}_{4} \mathrm{ClN}_{2} \mathrm{O}_{4} \mathrm{SNa}_{2}\left([\mathrm{M}+\mathrm{Na}]^{+}\right)$280.9376, found 280.9381.; EA: calcd. C, 24.46; H, 2.74; N, 9.51; found C, 24.43; H, 2.68; N, 9.56.

\section{$N, N^{\prime}$-bis(tert-butoxycarbonyl)sulfamide (BBS)}

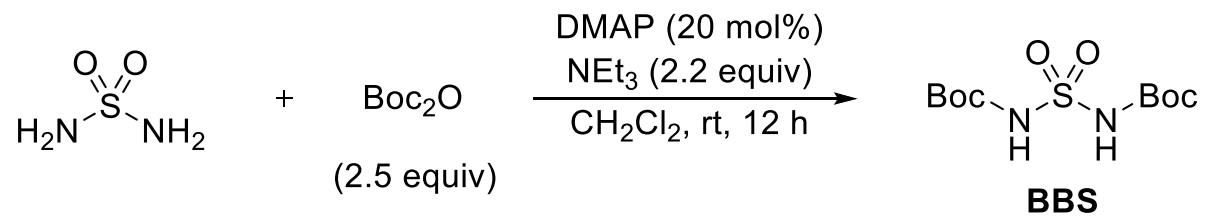

$\mathrm{NEt}_{3}(8842.0 \mathrm{mg}, 87.4 \mathrm{mmol})$ was added to a stirring solution of sulfamide $(3842.5 \mathrm{mg}, 40.0$ mmol) and DMAP $(978.0 \mathrm{mg}, 8.0 \mathrm{mmol})$ at $0{ }^{\circ} \mathrm{C}$ under a nitrogen atmosphere in $\mathrm{CH}_{2} \mathrm{Cl}_{2}(50$ $\mathrm{mL})$. Then, di-tert-butyl-dicarbonate $(21920.5 \mathrm{mg}, 100.4 \mathrm{mmol})$ solution in $\mathrm{CH}_{2} \mathrm{Cl}_{2}(80 \mathrm{~mL})$ was added dropwise over 10 minutes to the stirring solution. The mixture was warmed to room temperature and stirred for $12 \mathrm{~h}$. After stirring, the reaction mixture was concentrated in vacuo. The residual solid was diluted by EtOAc $(80 \mathrm{~mL})$ and washed by $1 \mathrm{M}$. $\mathrm{HCl}$ aq. $(3 \times 30 \mathrm{~mL})$, water $(30 \mathrm{~mL})$ and brine $(30 \mathrm{~mL})$. The organic layer was dried over $\mathrm{MgSO}_{4}$ and concentrated in vacuo. Washing the product by hexane gave the pure product $(8338.5 \mathrm{mg}, 71 \%)$ as a white solid. mp: 144.4-144.6 ${ }^{\circ} \mathrm{C}$ (dec.); ${ }^{1} \mathrm{H}$ NMR (400 MHz, DMSO- $\left.d_{6}\right) \delta 11.61$ (s, 2H), 1.41 (s, 18 $\mathrm{H}) ;{ }^{13} \mathrm{C}$ NMR (100 MHz, DMSO- $\left.d_{6}\right) \delta 149.8,81.8,27.6$; IR (ATR) 3184, 1753, 1728, 1423, 1364, 1234, 1136, 947, 820, $727 \mathrm{~cm}^{-1}$; HRMS (FAB-): $\mathrm{m} / z$ calcd. for $\mathrm{C}_{10} \mathrm{H}_{19} \mathrm{~N}_{2} \mathrm{O}_{6} \mathrm{~S}\left([\mathrm{M}-\mathrm{H}]^{-}\right)$ 295.0964, found 295.0966. 
sodium(tert-butoxycarbonyl)( $N$-(tert-butoxycarbonyl)- $N$-chlorosulfamoyl)amide (Chloramine-BBS)<smiles>CC(C)(C)OC(=O)NS(=O)(=O)N[C@H](O)OC(=O)OCc1ccccc1</smiles>

BBS

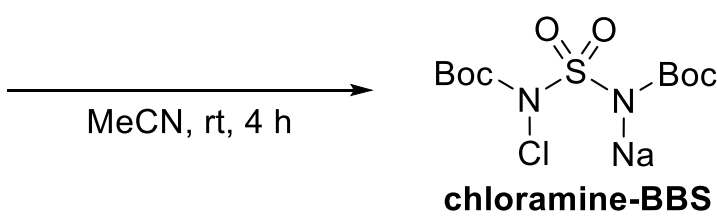

$96 \%$

BBS (2966.0 mg, $10.0 \mathrm{mmol})$ and sodium hypochlorite pentahydrate $(1649.0 \mathrm{mg}, 10.0 \mathrm{mmol})$ were stirred at room temperature in $\mathrm{MeCN}(50 \mathrm{~mL})$ for $4 \mathrm{~h}$. After stirring, the reaction mixture was concentrated in vacuo. Washing the product by $\mathrm{Et}_{2} \mathrm{O}$ gave the pure product $(3343.9 \mathrm{mg}$, 96\%) as a white solid. mp: $288.1-289.0{ }^{\circ} \mathrm{C} ;{ }^{1} \mathrm{H}$ NMR (400 MHz, Acetone- $\left.d_{6}\right) \delta 1.484(\mathrm{~s}, 9 \mathrm{H})$, 1.359 (s, 9H); IR (ATR) 2978, 1694, 1638, 1327, 1287, 1151, 1105, 851, $756 \mathrm{~cm}^{-1}$; Analysis: $\mathrm{C}_{10} \mathrm{H}_{18} \mathrm{ClN}_{2} \mathrm{NaO}_{6} \mathrm{~S} \bullet 1 / 2 \mathrm{H}_{2} \mathrm{O}$ Calcd: C, 33.2; H, 5.29; N, 7.74, Found: C, 33.26; H, 5.35; N, 7.88. 


\section{NMR Spectra}

$N, N^{\prime}$-(4-phenylbutane-1,2-diyl)bis(2-nitrobenzenesulfonamide) (3a)<smiles>[N-]S(=O)(=O)CC(CCc1ccccc1)NS(=O)(=O)[O-]</smiles>

${ }^{1} \mathrm{H}$ NMR $\left(400 \mathrm{MHz}, \mathrm{CDCl}_{3}\right)$

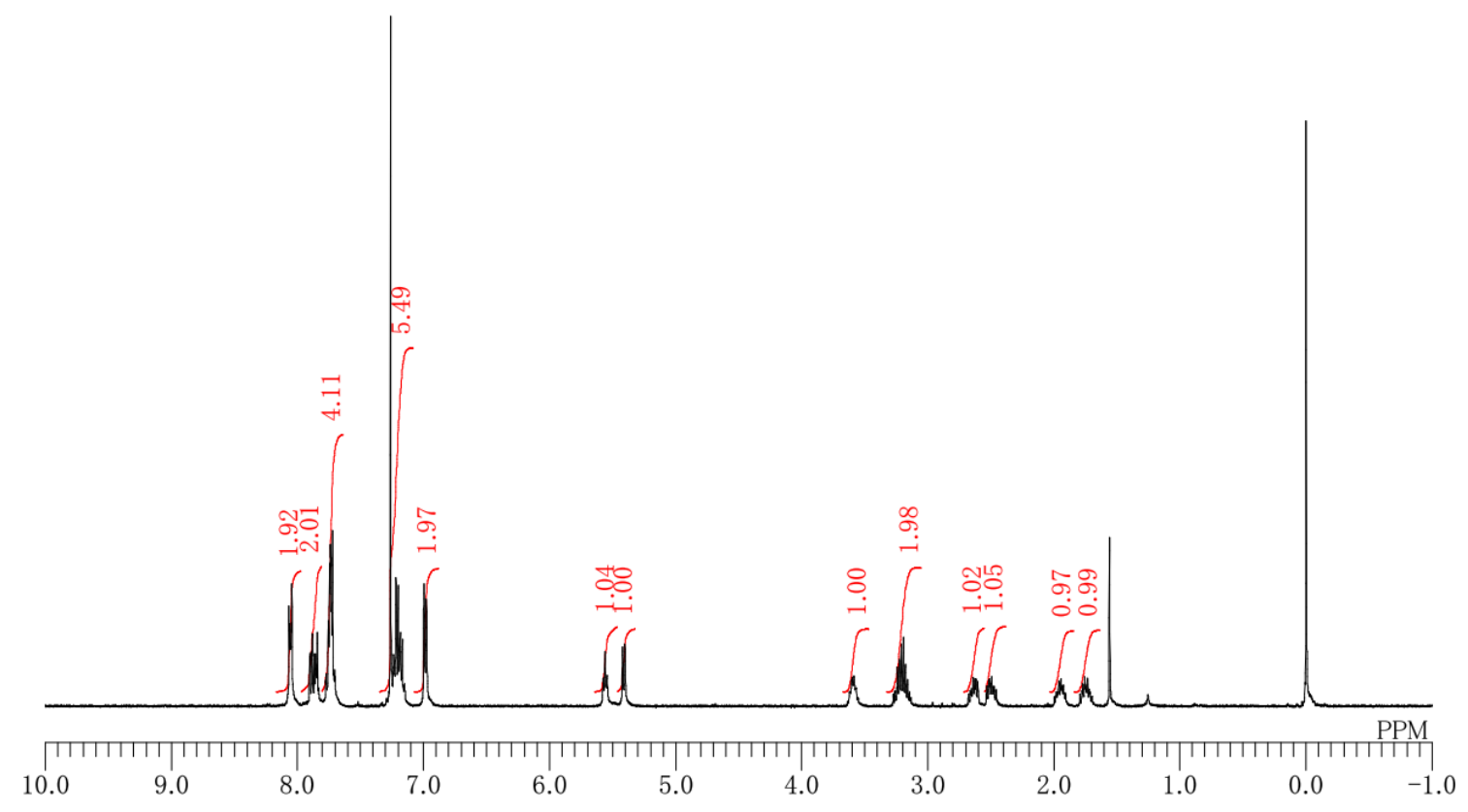

${ }^{13} \mathrm{C}$ NMR (100 MHz, $\left.\mathrm{CDCl}_{3}\right)$
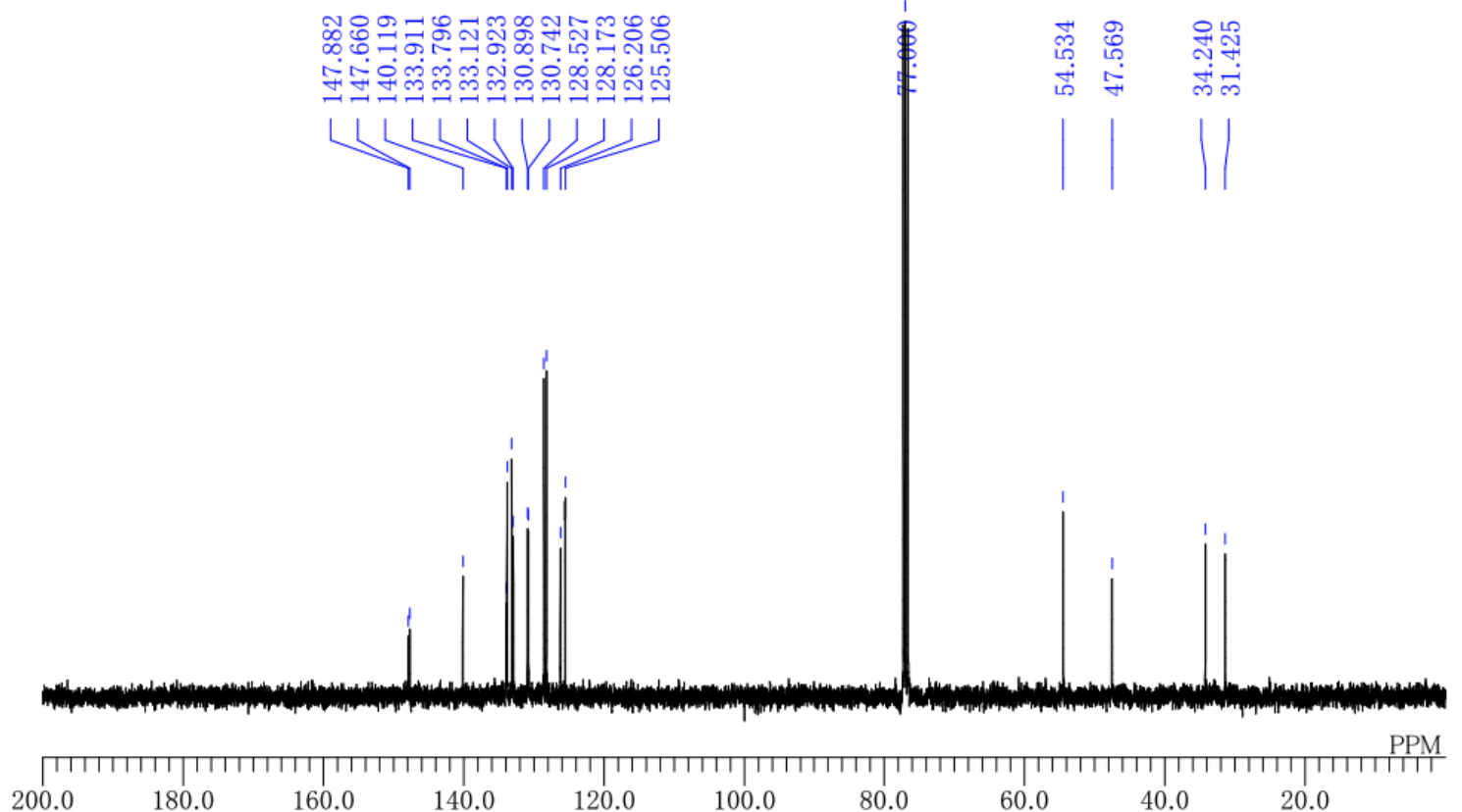
$N, N^{\prime}$-(3-phenylpropane-1,2-diyl)bis(2-nitrobenzenesulfonamide) (3b)<smiles></smiles>

${ }^{1} \mathrm{H}$ NMR (400 MHz, DMSO- $\left.d_{6}\right)$

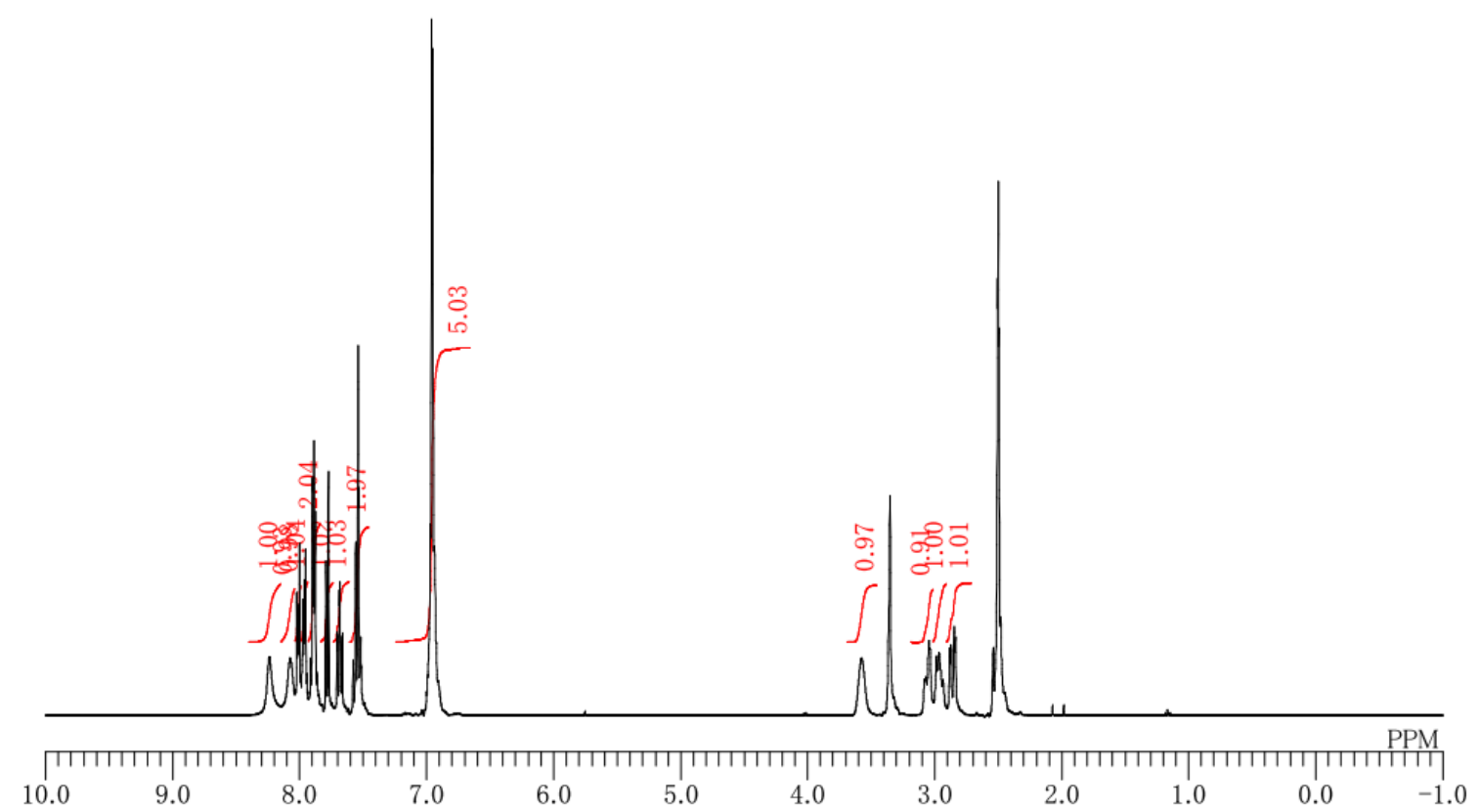

${ }^{13} \mathrm{C}$ NMR (100 MHz, DMSO- $d_{6}$ )

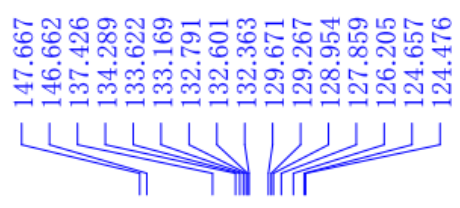

艹ृ

เค
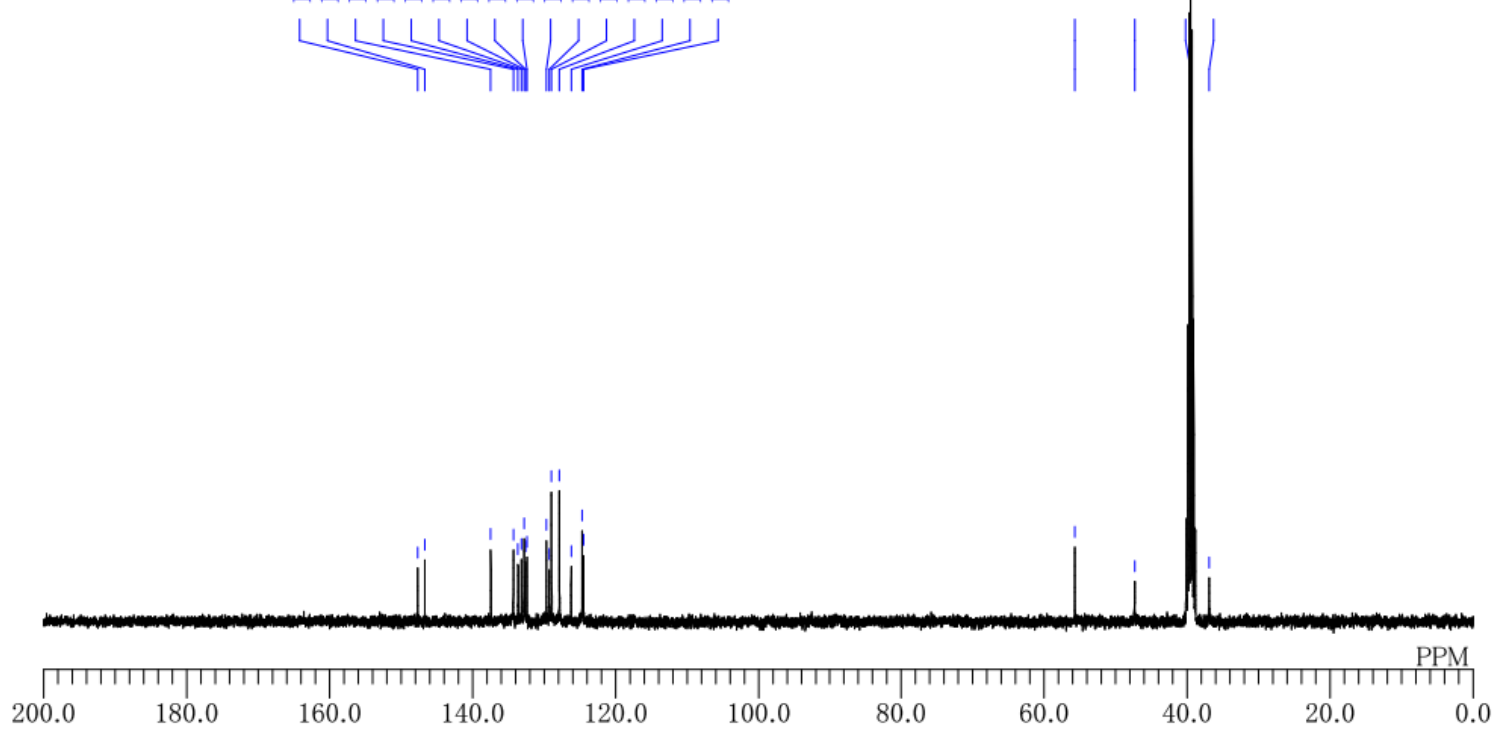
$N, N^{\prime}$-(octane-1,2-diyl)bis(2-nitrobenzenesulfonamide) (3c)

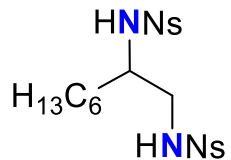

${ }^{1} \mathrm{H}$ NMR (400 MHz, DMSO- $\left.d_{6}\right)$

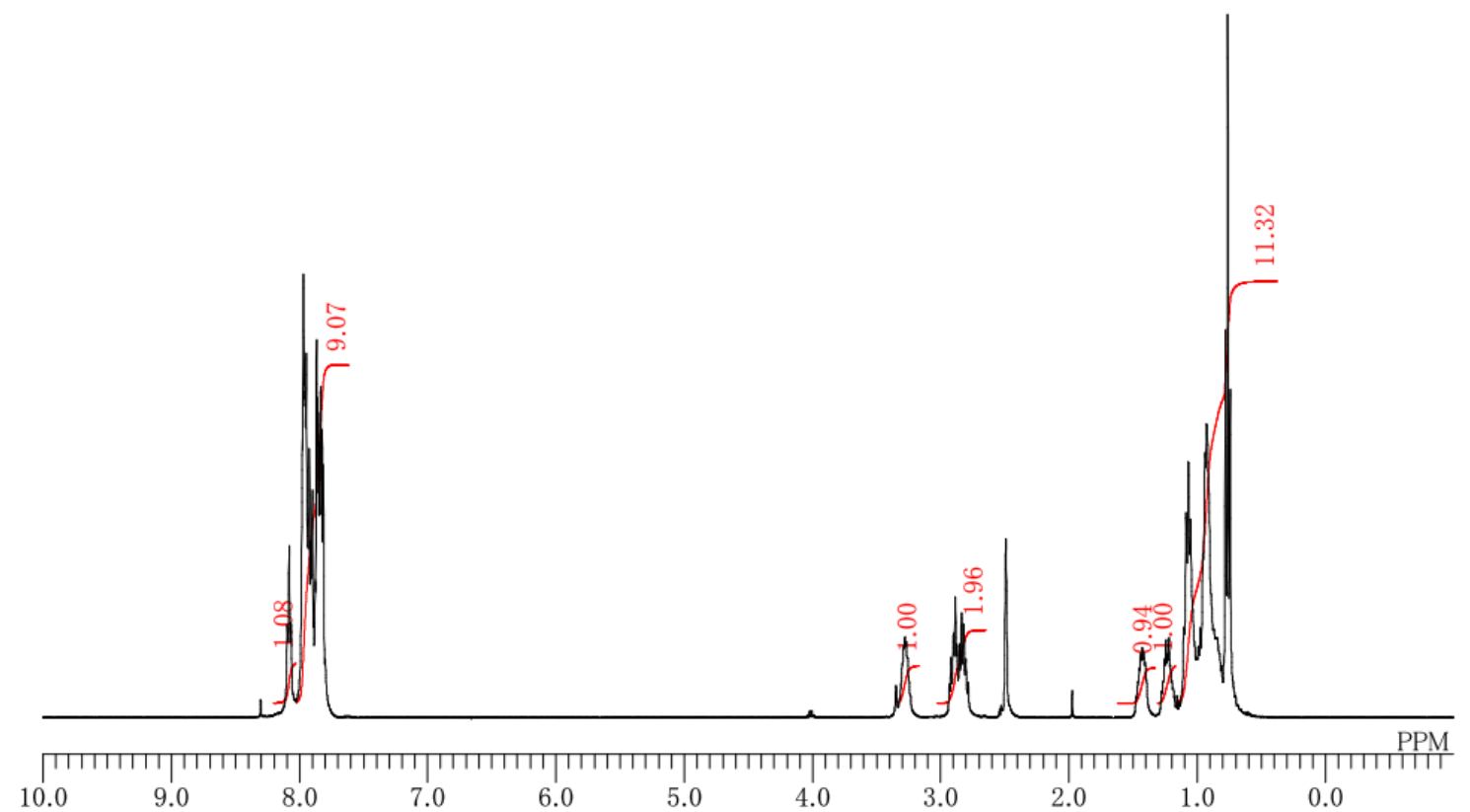

${ }^{13} \mathrm{C}$ NMR (100 MHz, DMSO- $\left.d_{6}\right)$
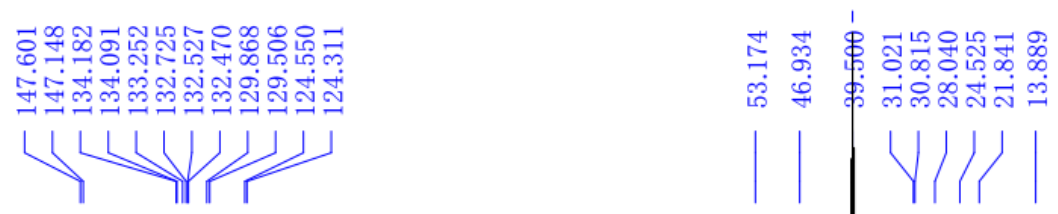

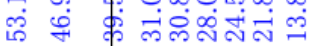
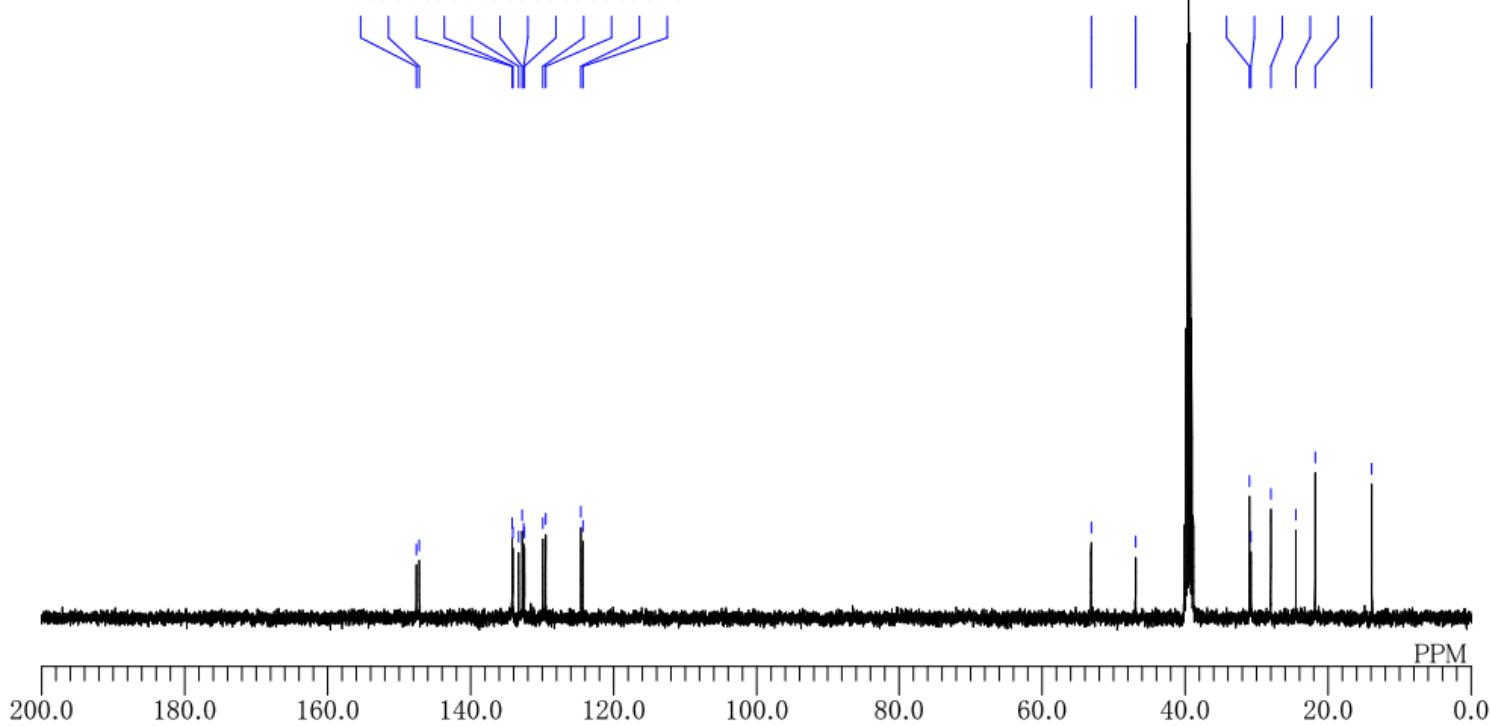
$N, N^{\prime}$-(7-bromoheptane-1,2-diyl)bis(2-nitrobenzenesulfonamide) (3d)

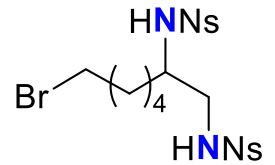

${ }^{1} \mathrm{H}$ NMR $\left(400 \mathrm{MHz}, \mathrm{CDCl}_{3}\right)$

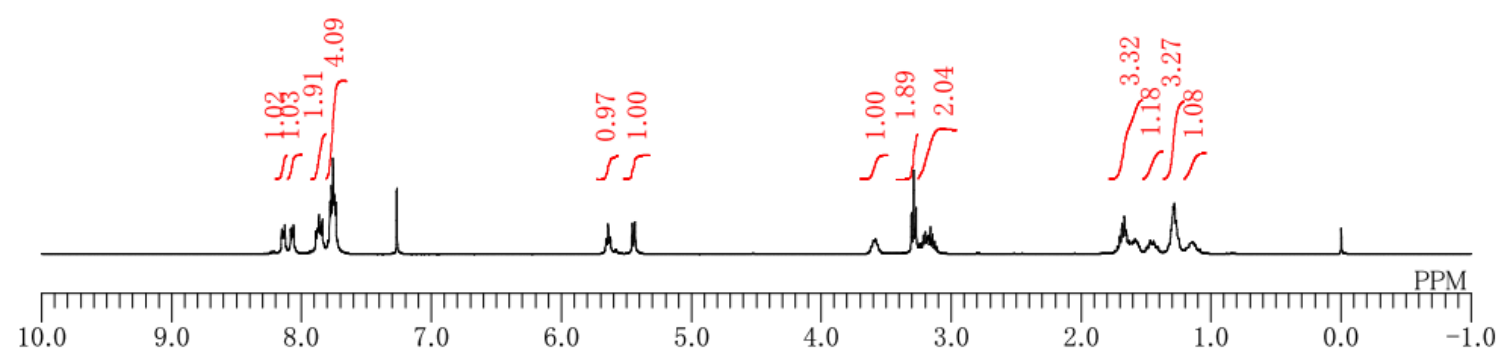

${ }^{13} \mathrm{C} \mathrm{NMR}\left(100 \mathrm{MHz}, \mathrm{CDCl}_{3}\right)$
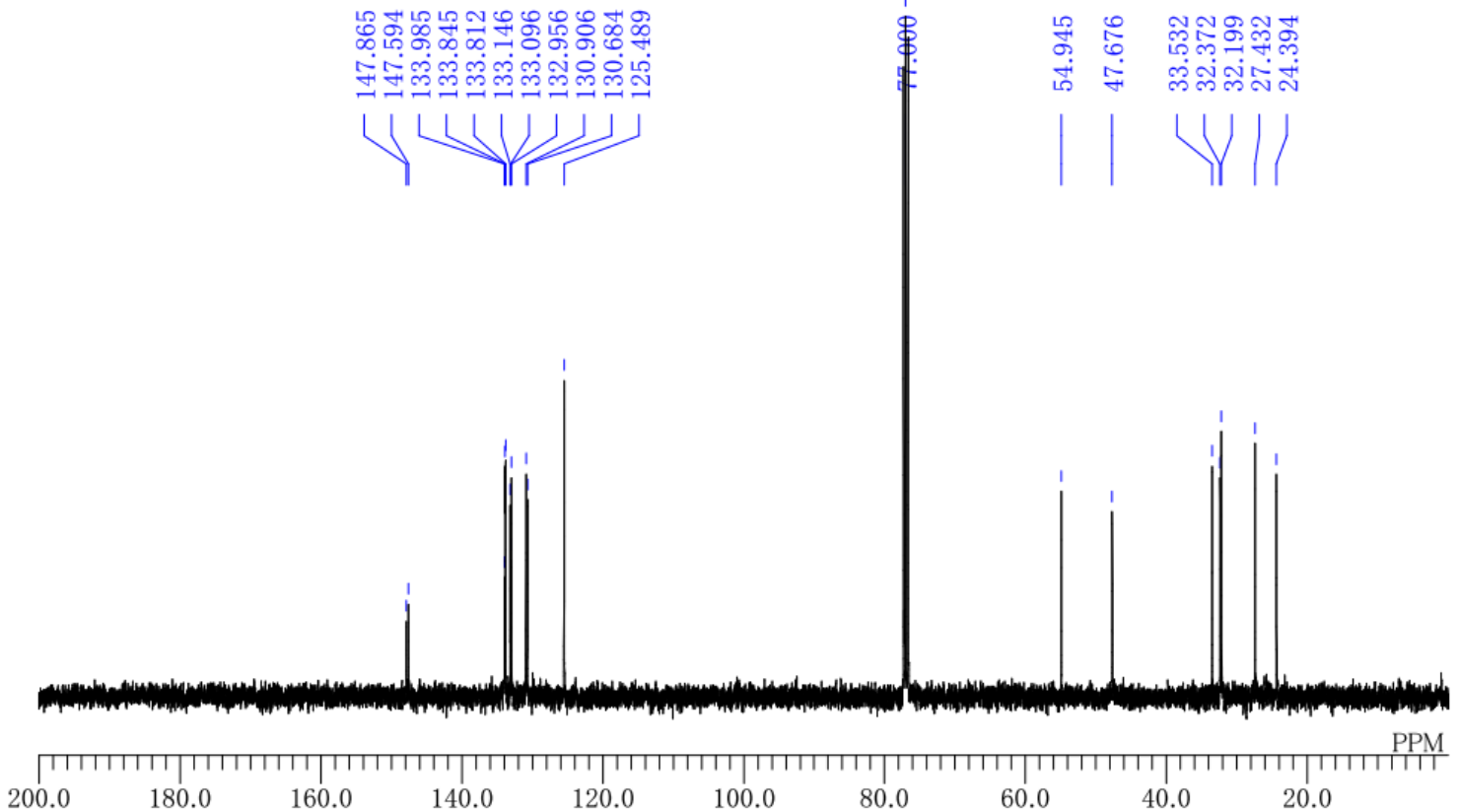
$N, N^{\prime}$-(methyl undecanoate-10,11-diyl)bis(2-nitrobenzenesulfonamide) (3e)<smiles>COC(=O)CC(N)CNS(N)(=O)=O</smiles>

${ }^{1} \mathrm{H}$ NMR (400 MHz, $\mathrm{CDCl}_{3}$ )

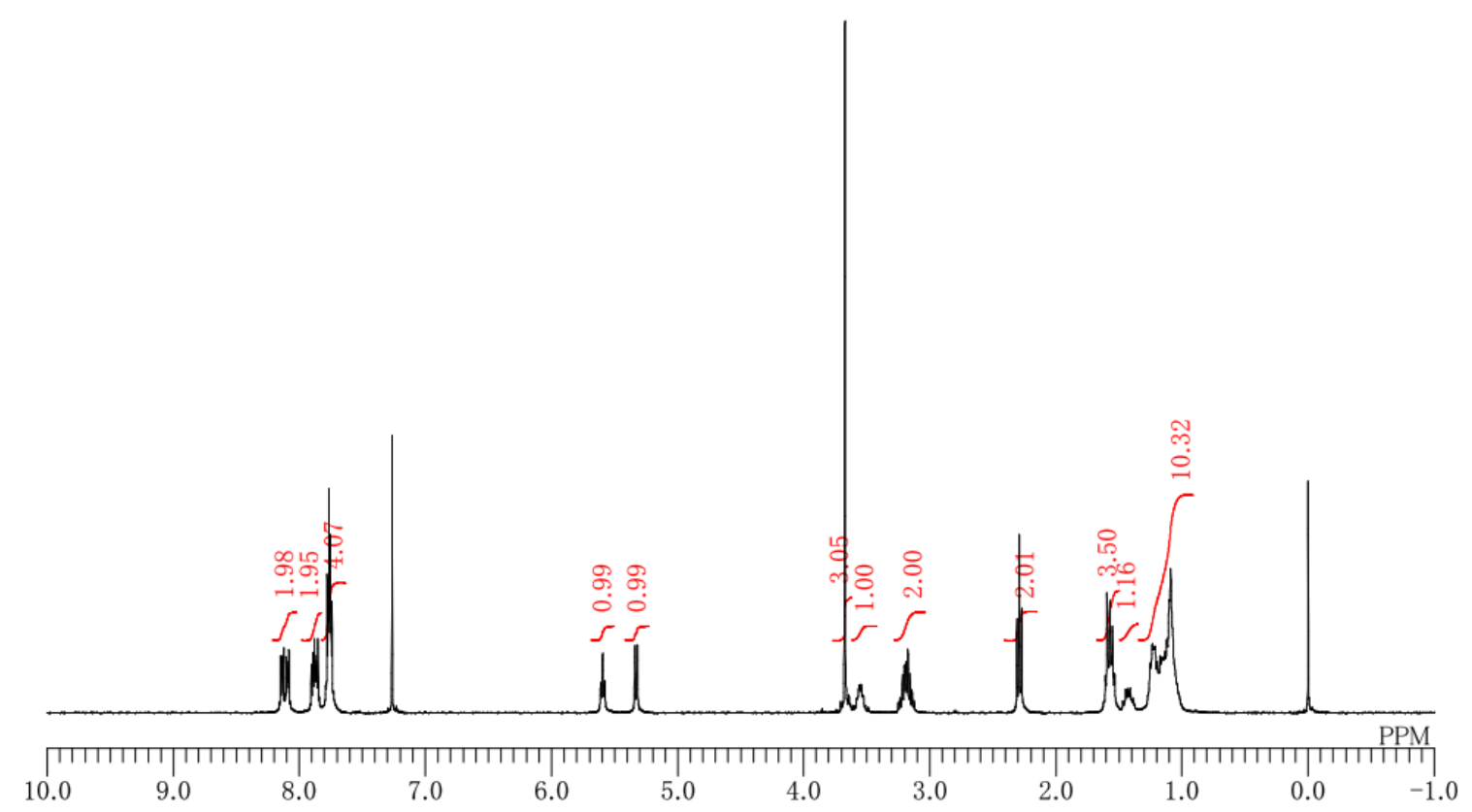

${ }^{13} \mathrm{C}$ NMR (100 MHz, $\mathrm{CDCl}_{3}$ )
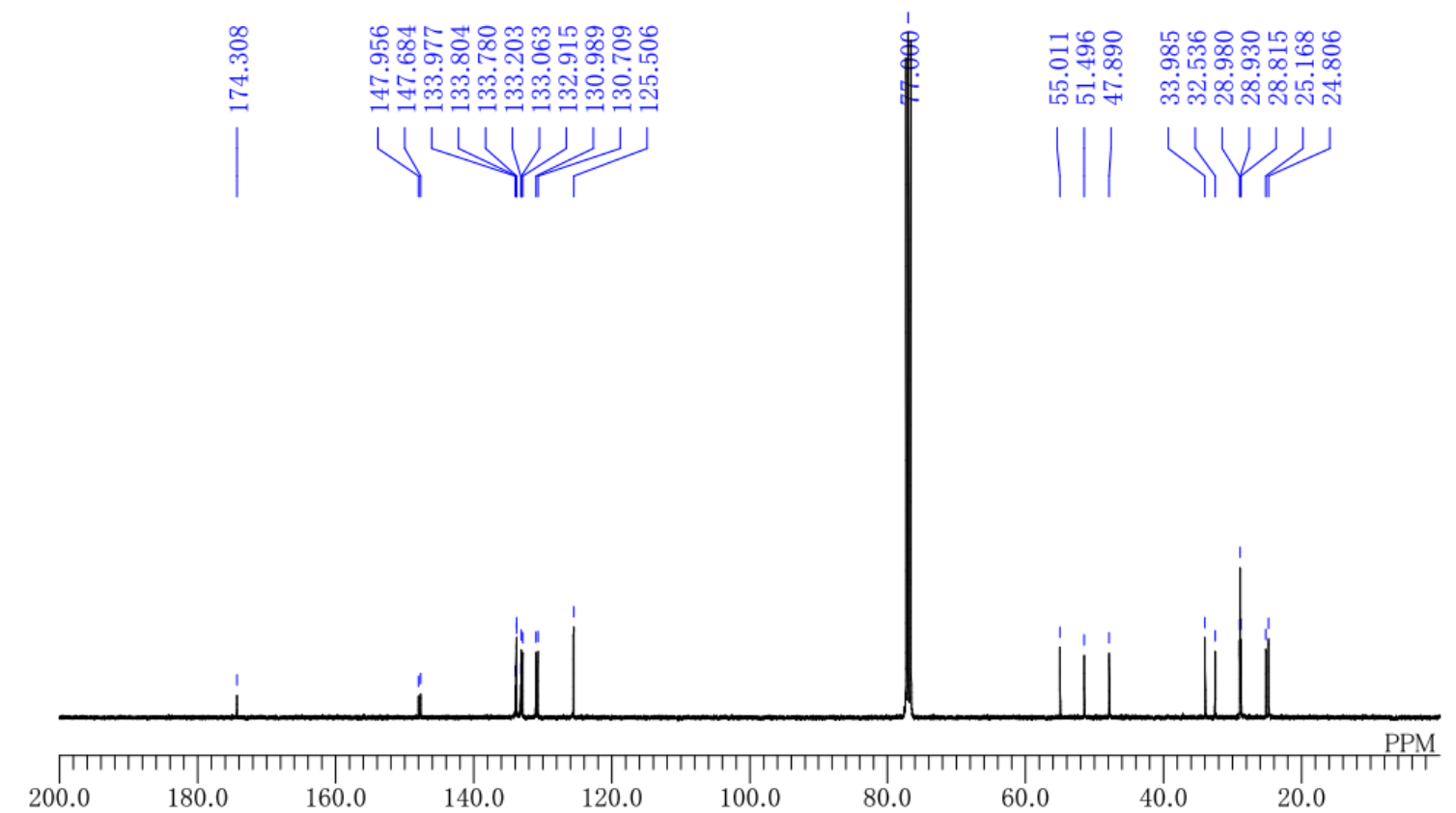
$N, N^{\prime}$-(3-hydroxypropane-1,2-diyl)bis(2-nitrobenzenesulfonamide) (3f)<smiles>[N-]S(=O)(=O)CC(CO)CNS(=O)(=O)[O-]</smiles>

${ }^{1} \mathrm{H}$ NMR $\left(400 \mathrm{MHz}, \mathrm{CDCl}_{3}\right)$

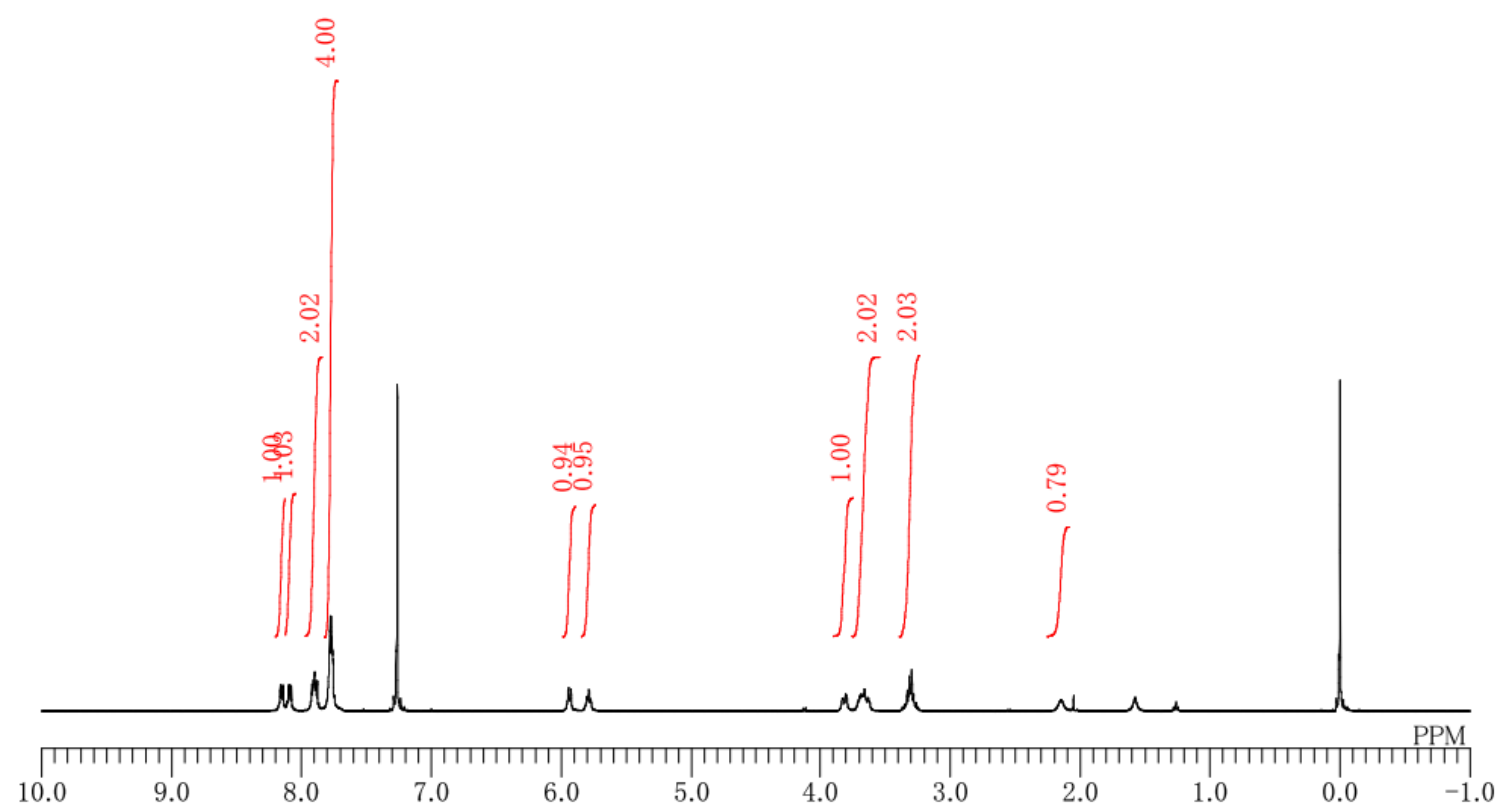

${ }^{13} \mathrm{C}$ NMR $\left(100 \mathrm{MHz}, \mathrm{CDCl}_{3}\right)$

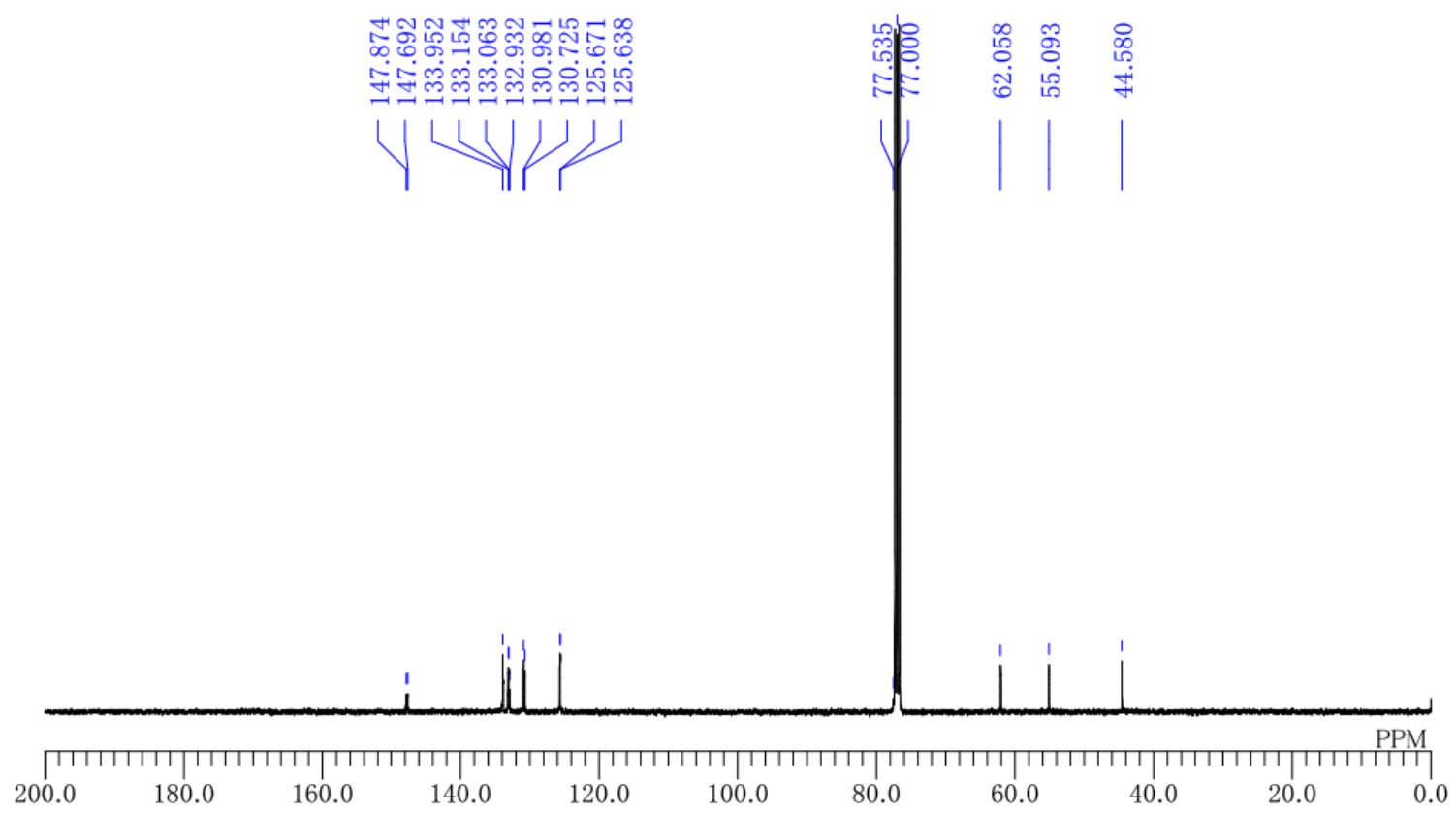


$N, N^{\prime}$-(7-hydroxyheptane-1,2-diyl)bis(2-nitrobenzenesulfonamide) (3g)<smiles>CC(CN[N+](=O)[O-])CN(C)S(=O)(=O)[O-]</smiles>

${ }^{1} \mathrm{H}$ NMR (400 MHz, $\left.\mathrm{CDCl}_{3}\right)$

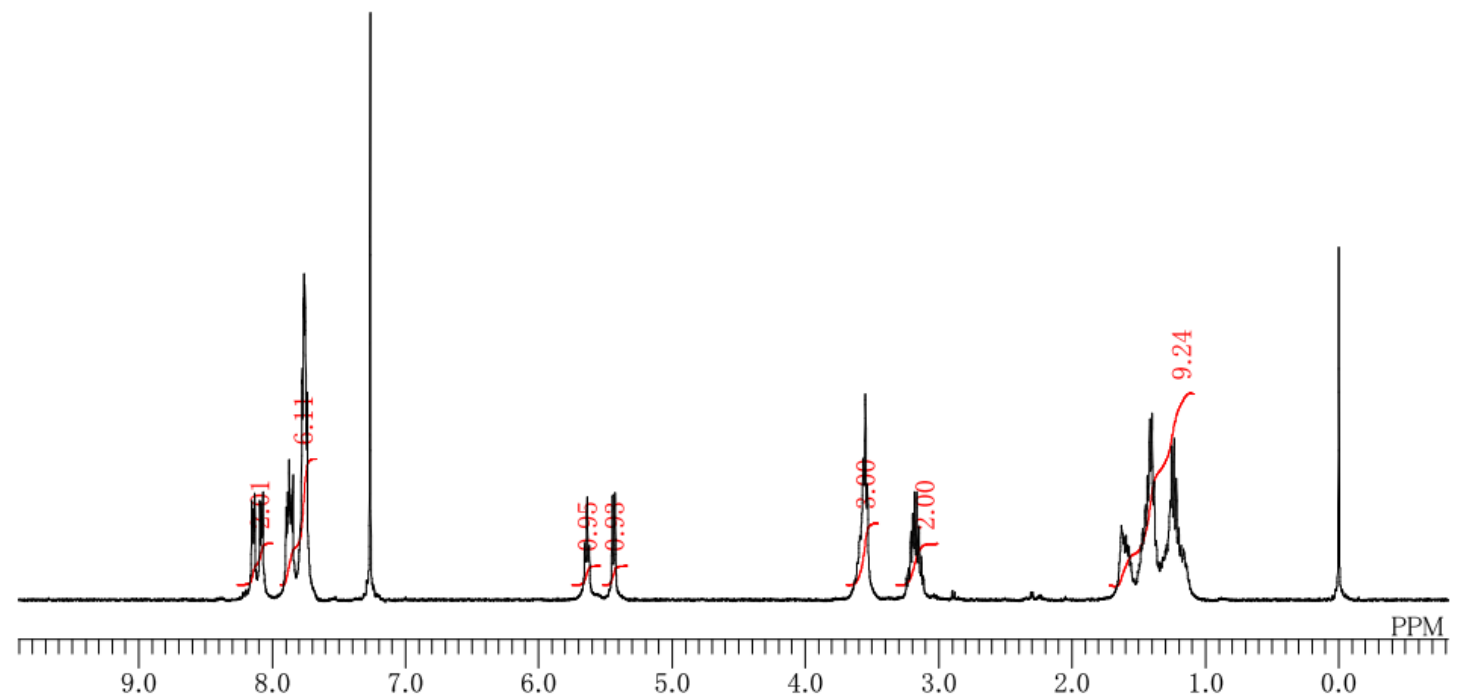

${ }^{13} \mathrm{C}$ NMR $\left(100 \mathrm{MHz}, \mathrm{CDCl}_{3}\right)$

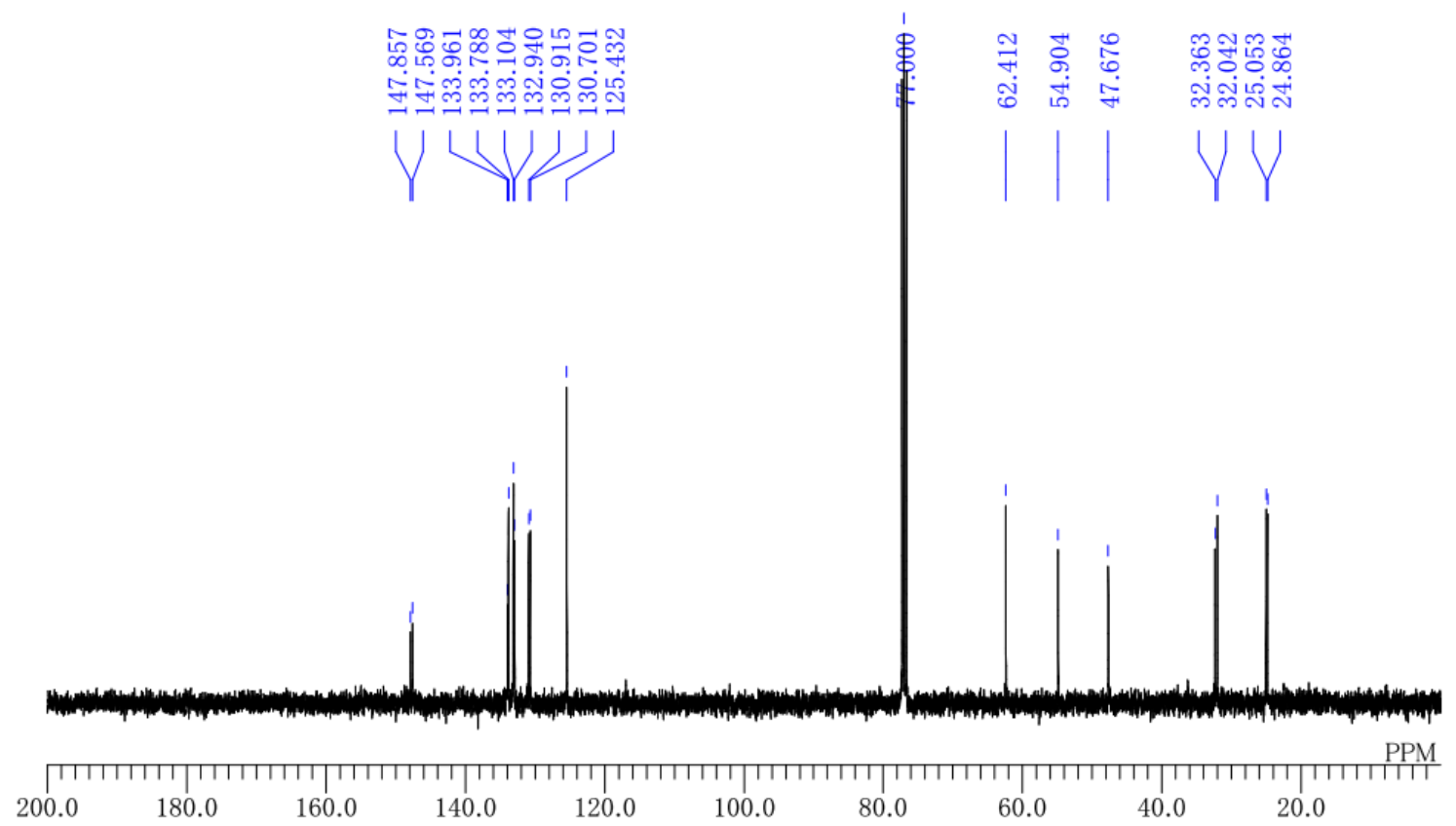


$N, N^{\prime}$-(undecanal-10,11-diyl)bis(2-nitrobenzenesulfonamide) (3h)<smiles>CC(C=O)CN[N+](=O)[O-]</smiles>

${ }^{1} \mathrm{H}$ NMR (400 MHz, $\mathrm{CDCl}_{3}$ )

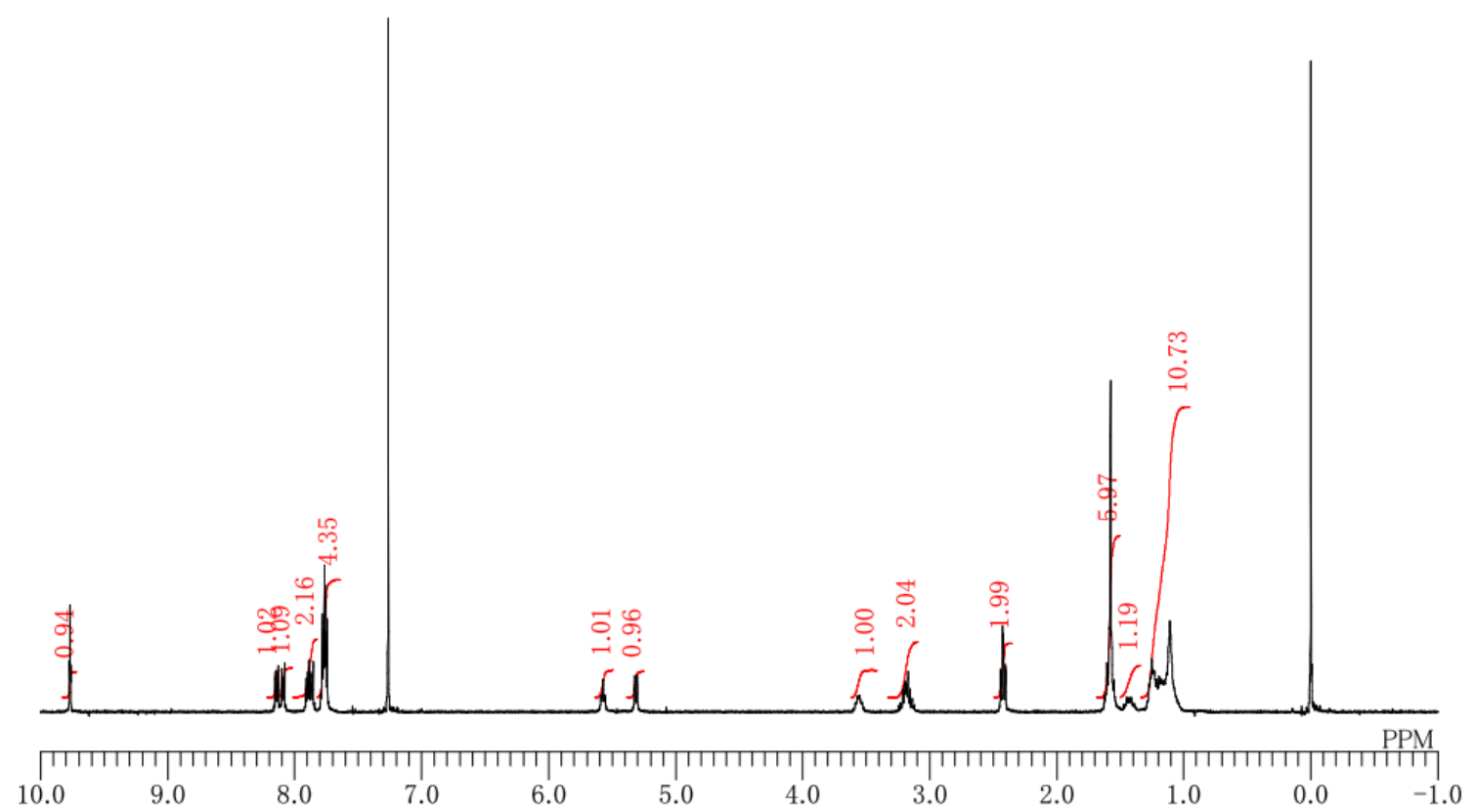

${ }^{13} \mathrm{C}$ NMR $\left(100 \mathrm{MHz}, \mathrm{CDCl}_{3}\right)$

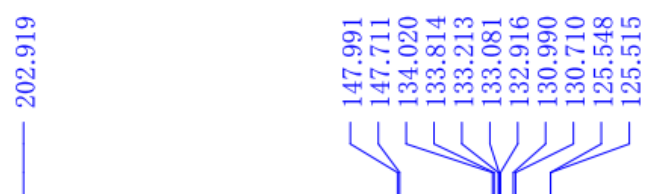

ब

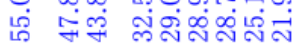
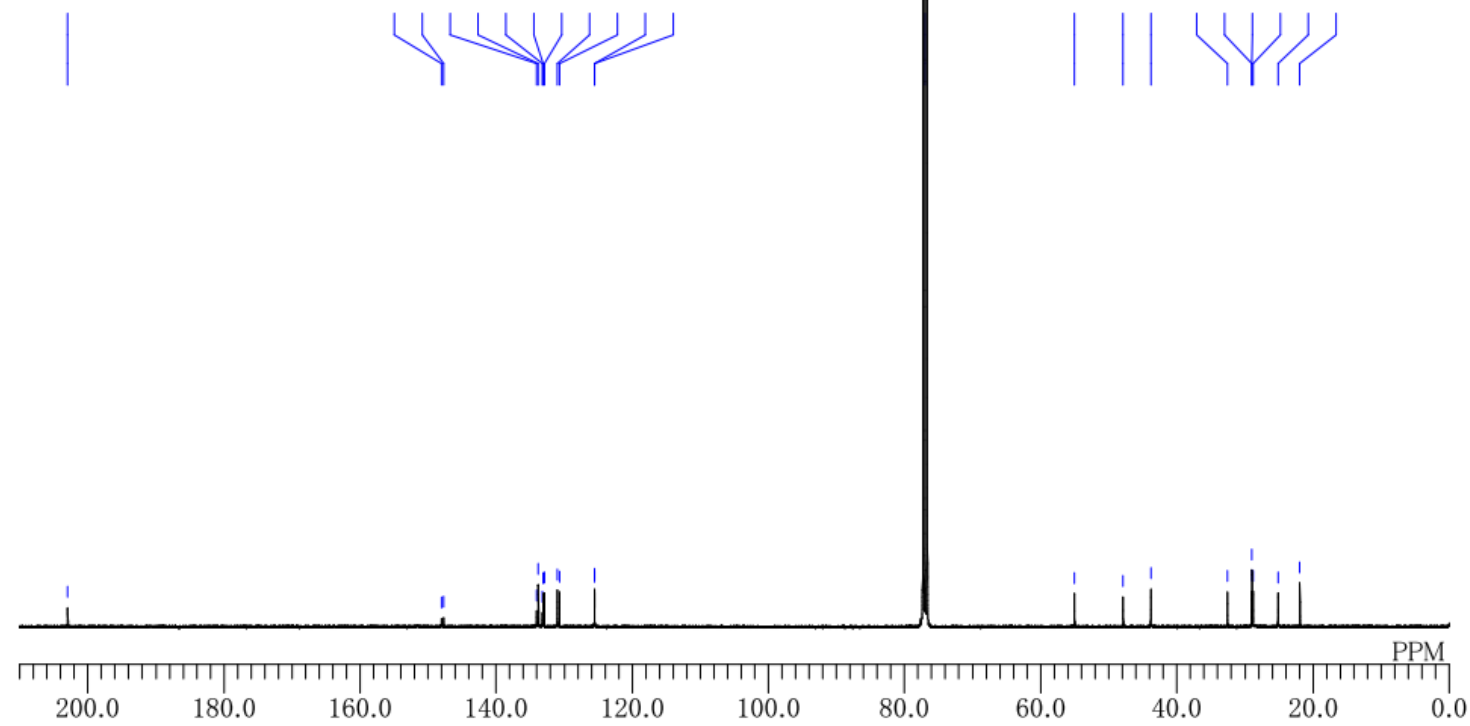
$N, N^{\prime}$-(1-(2-oxopyrrolidin-1-yl)ethane-1,2-diyl)bis(2-nitrobenzenesulfonamide) (3i)<smiles>NS(=O)(=O)CC(N1CCCC1=O)[N+](=O)[O-]</smiles>

${ }^{1} \mathrm{H}$ NMR (400 MHz, DMSO- $\left.d_{6}\right)$

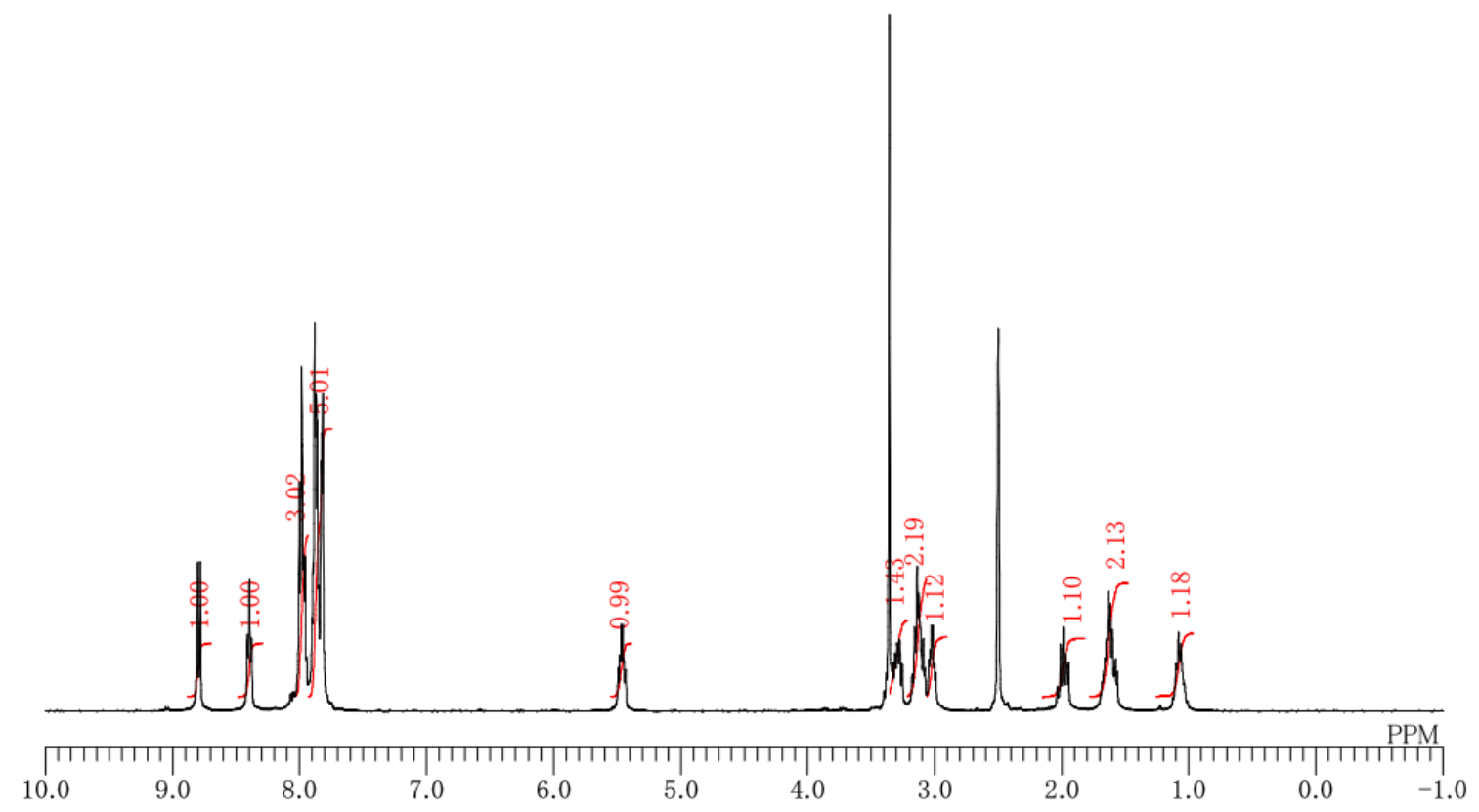

${ }^{13} \mathrm{C}$ NMR $\left(100 \mathrm{MHz}\right.$, DMSO- $\left.d_{6}\right)$
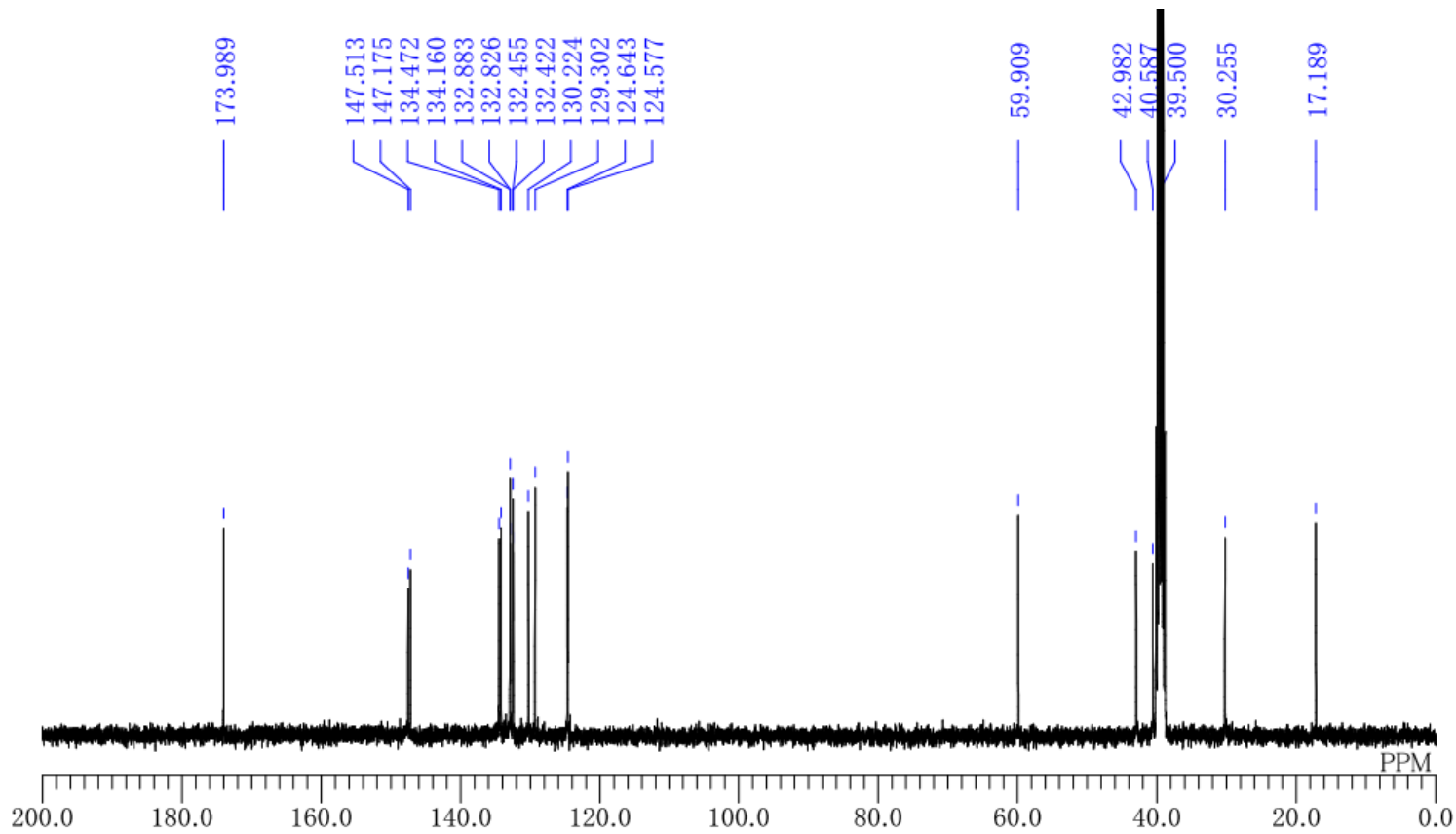
$N, N^{\prime}$-(3-((4-methylphenyl)sulfonamido)propane-1,2-diyl)bis(2-nitrobenzenesulfonamide) (3j)

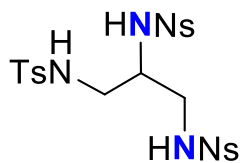

${ }^{1} \mathrm{H}$ NMR $\left(400 \mathrm{MHz}, \mathrm{CDCl}_{3}\right)$

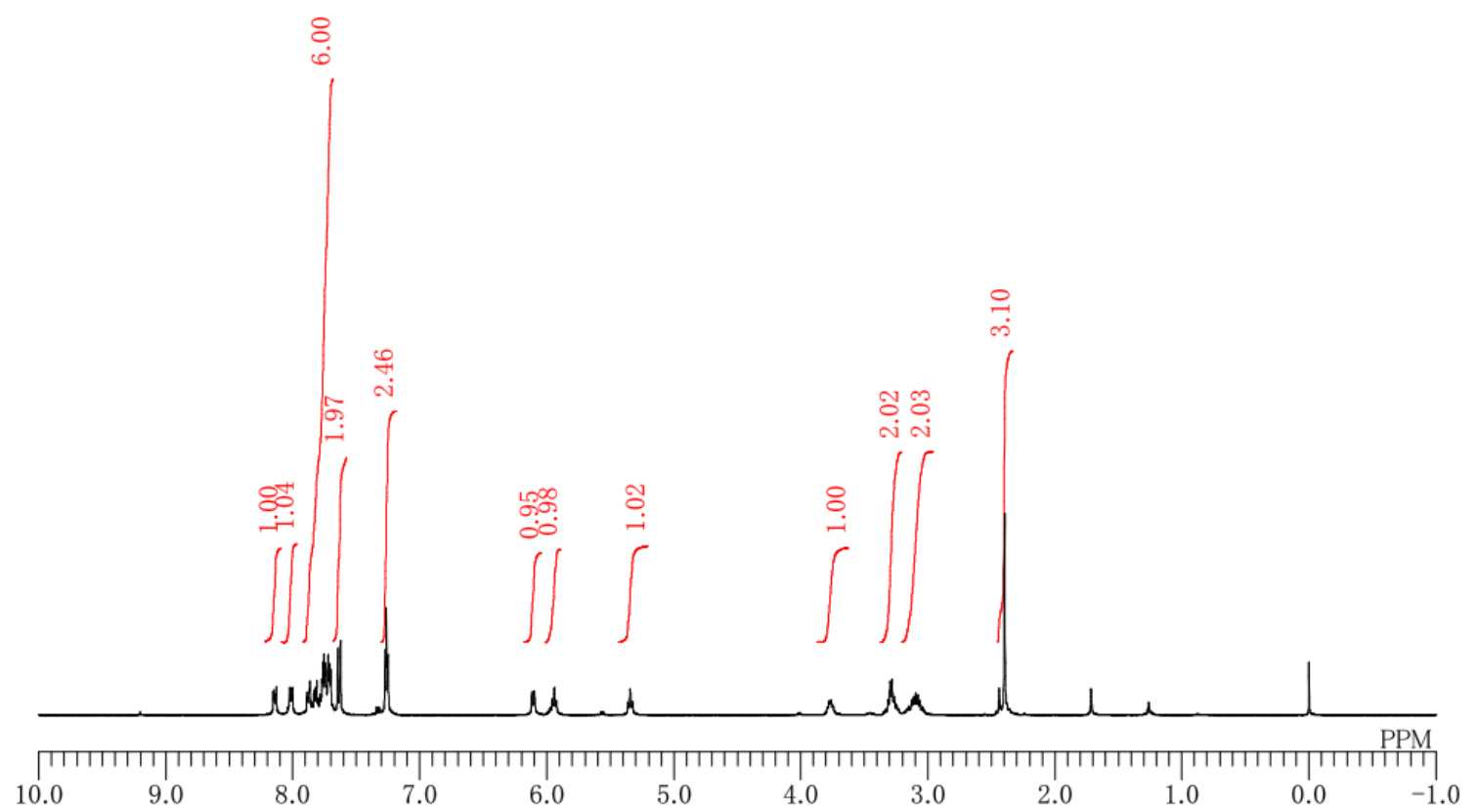

${ }^{13} \mathrm{C}$ NMR $\left(100 \mathrm{MHz}, \mathrm{CDCl}_{3}\right)$
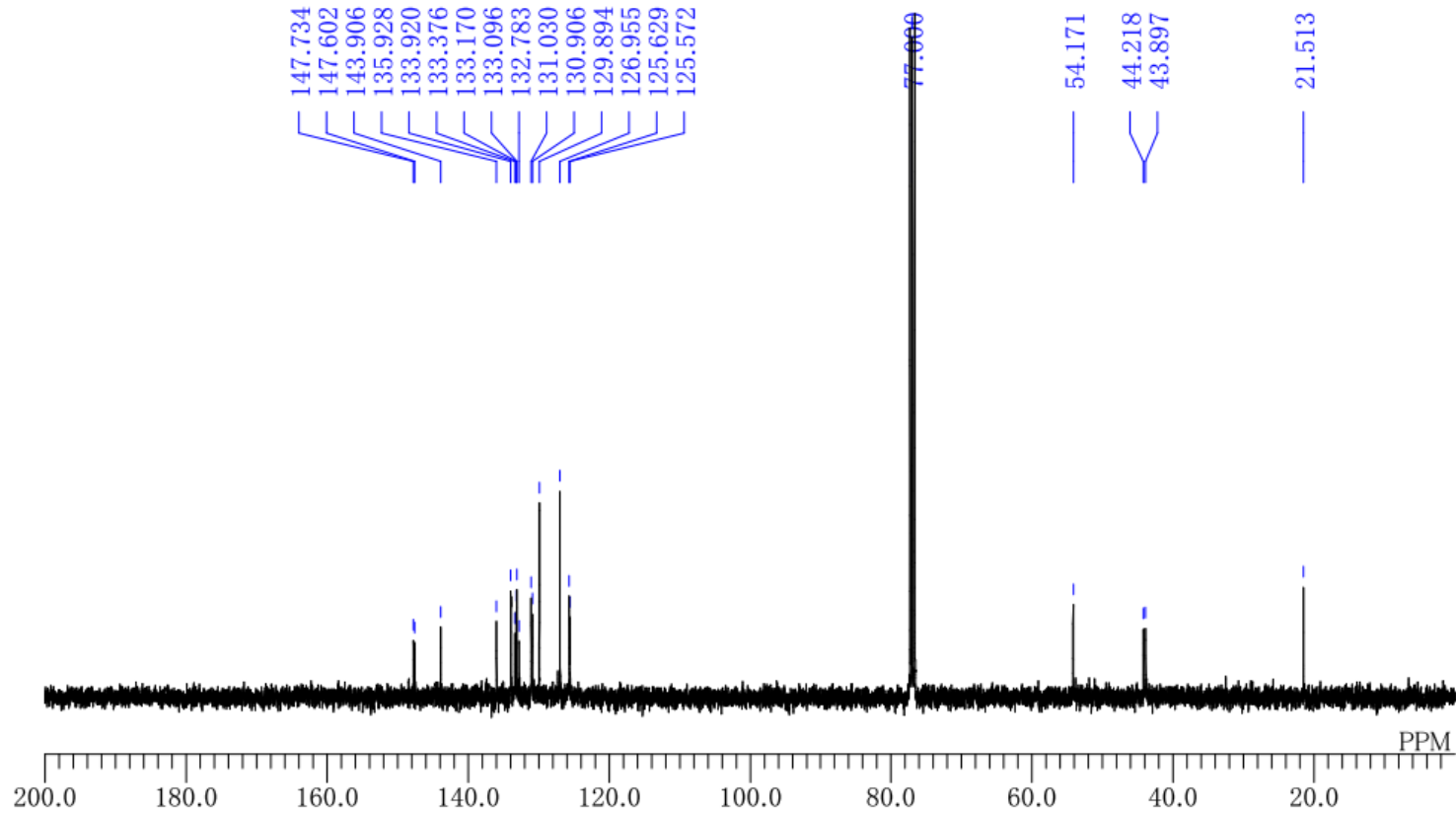
$N, N^{\prime}$-(1-phenylethane-1,2-diyl)bis(2-nitrobenzenesulfonamide) (3k)<smiles>CS(=O)(=O)NCC(c1ccccc1)c1ccccc1</smiles>

${ }^{1} \mathrm{H}$ NMR (400 MHz, DMSO- $\left.d_{6}\right)$

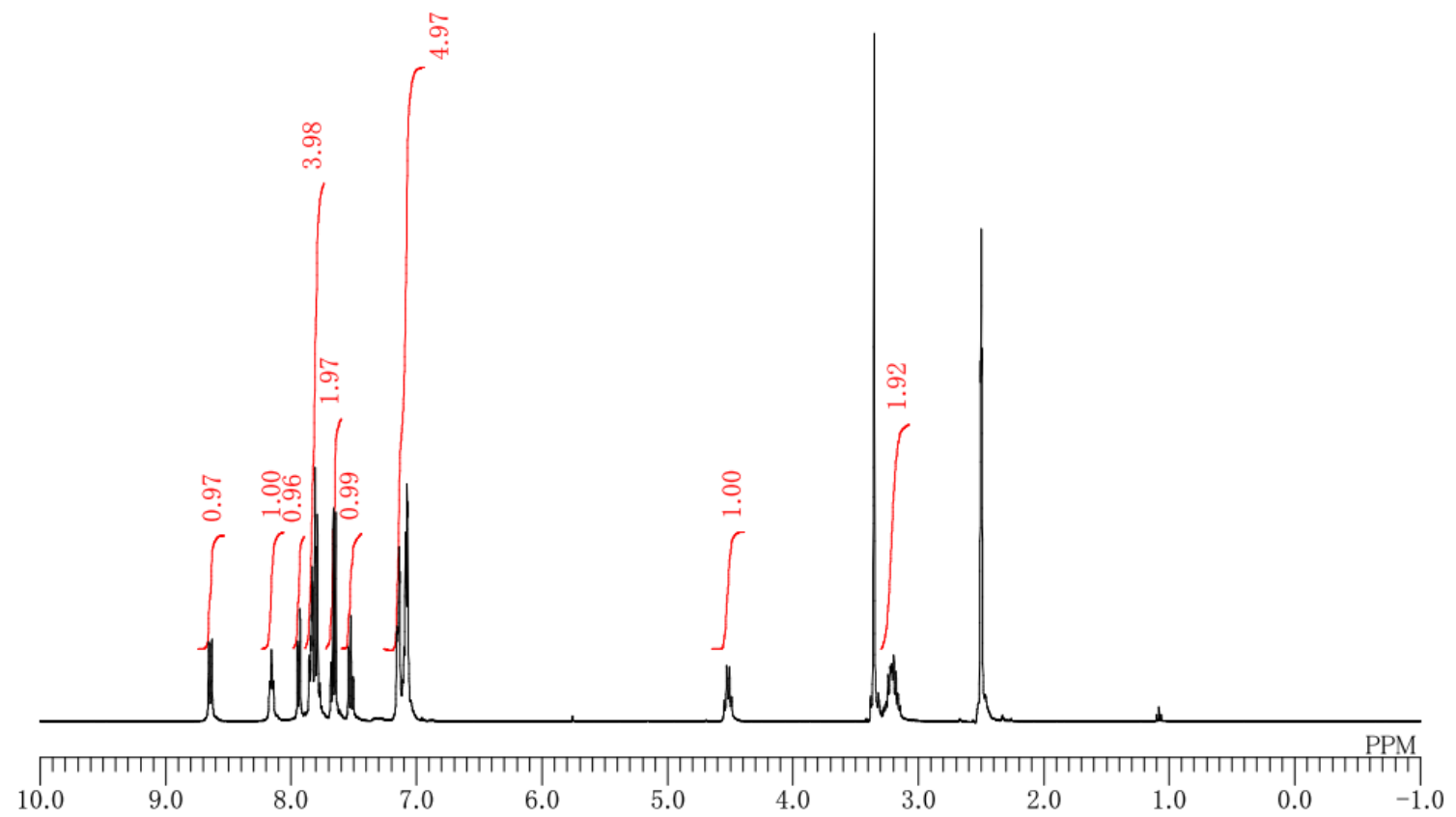

${ }^{13} \mathrm{C}$ NMR $\left(100 \mathrm{MHz}\right.$, DMSO- $\left.d_{6}\right)$
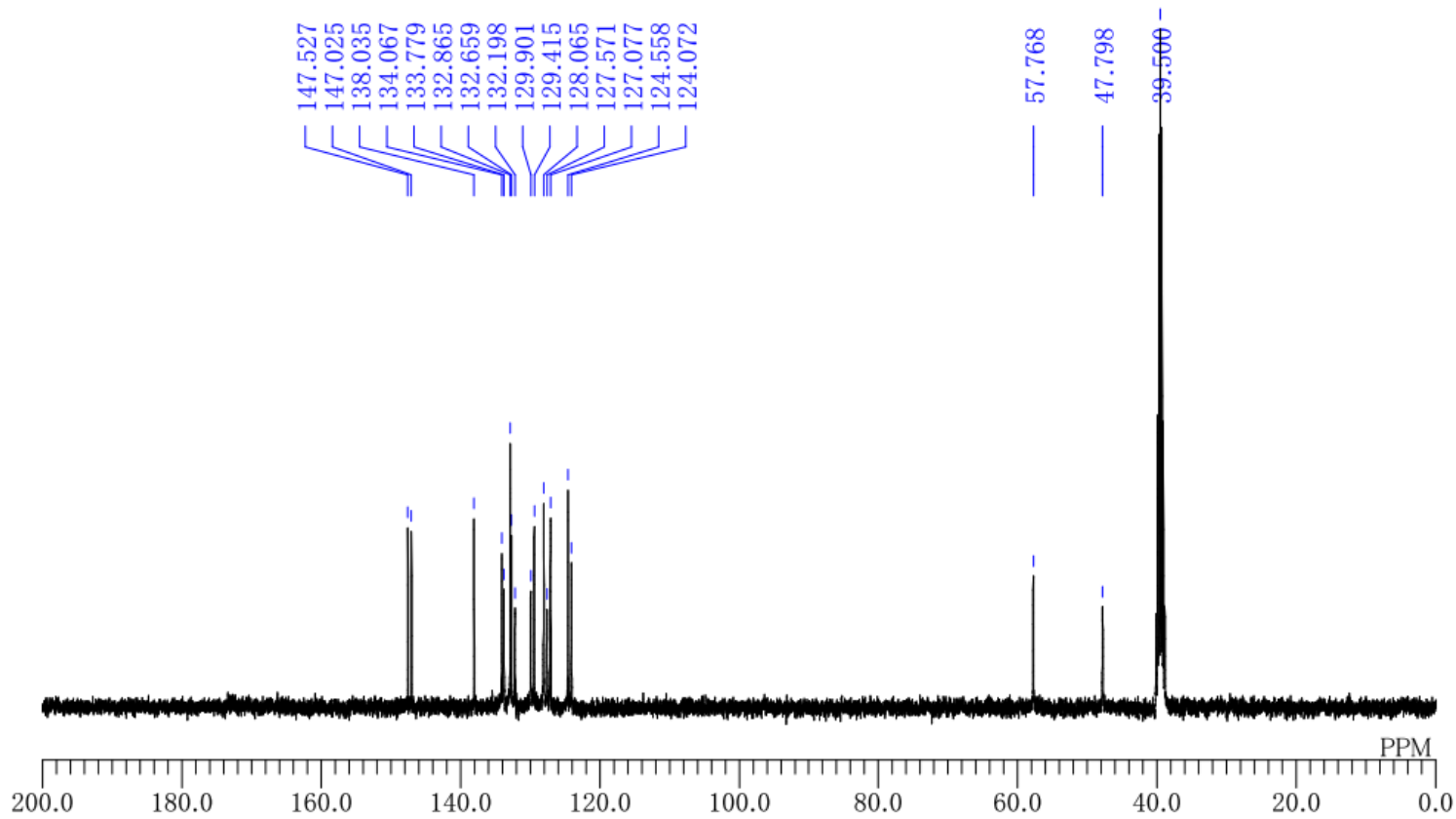
$N, N^{\prime}$-(1-(4-fluorophenyl)ethane-1,2-diyl)bis(2-nitrobenzenesulfonamide) (3I)<smiles>NC(CS(N)(=O)=O)c1ccc(F)cc1</smiles>

${ }^{1} \mathrm{H}$ NMR (400 MHz, DMSO- $d_{6}$ )
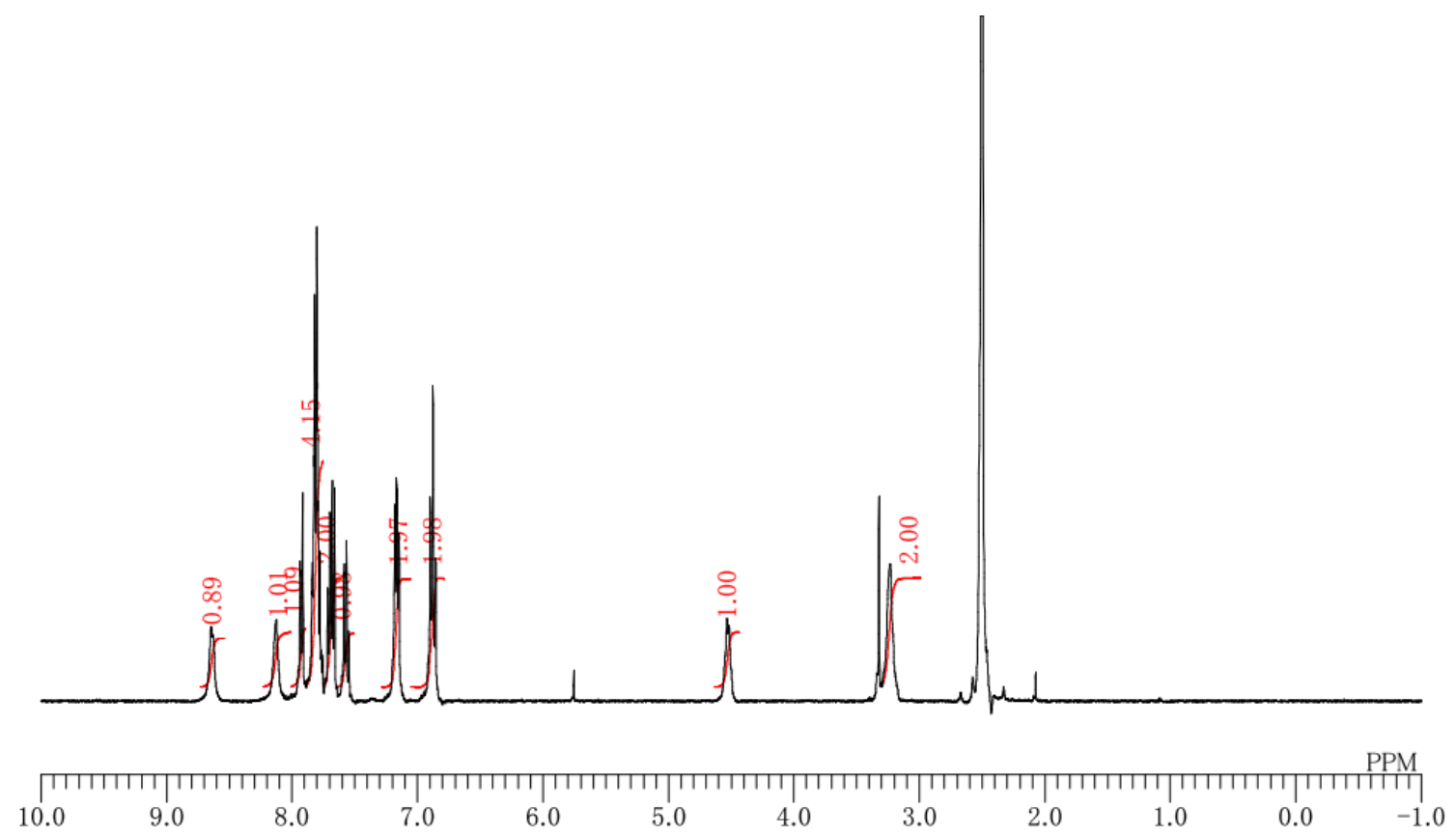

${ }^{13} \mathrm{C}$ NMR (100 MHz, DMSO- $\left.d_{6}\right)$
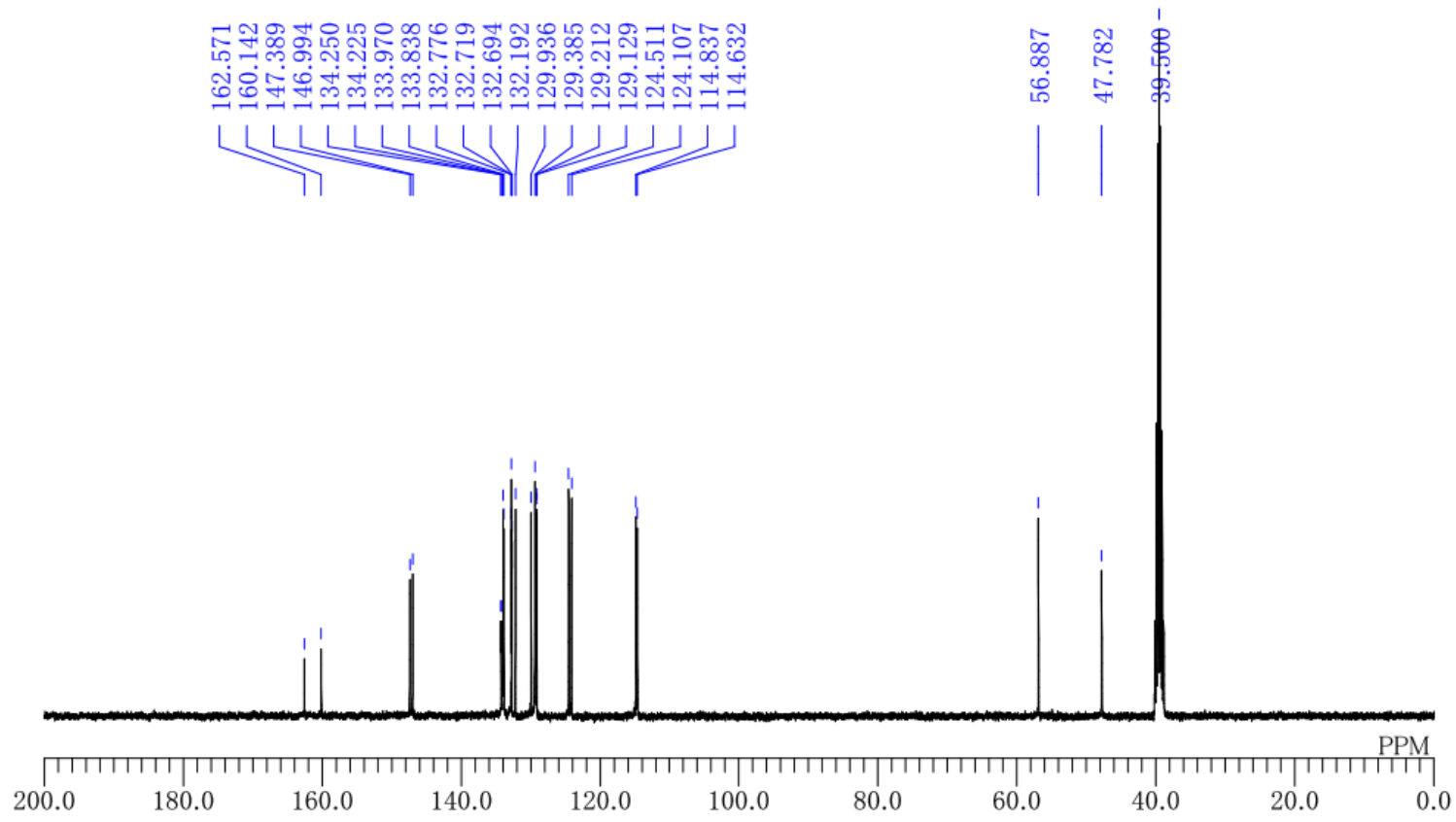
${ }^{19}$ F NMR (377 MHz, DMSO- $d_{6}$ )

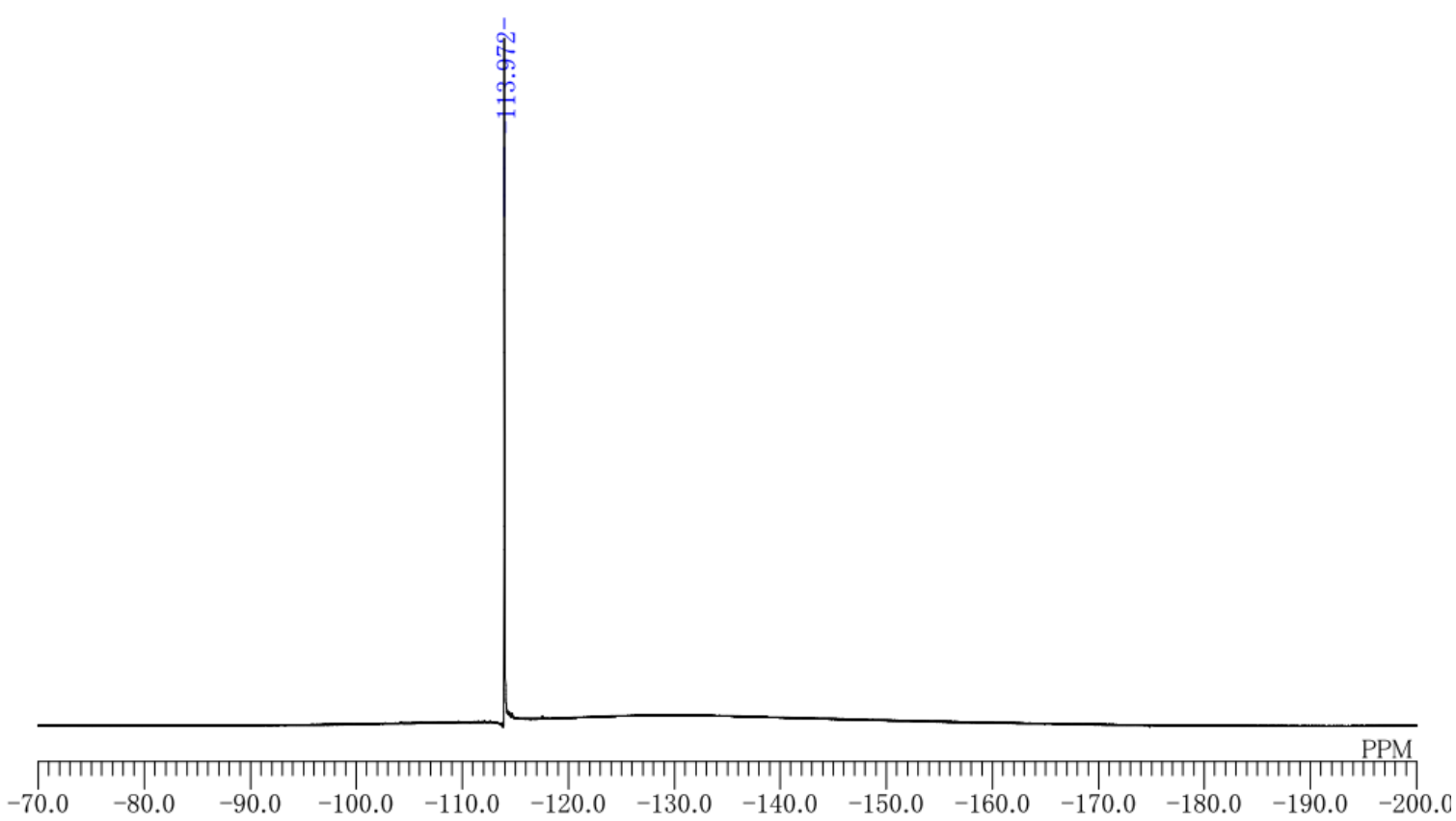


$N, N^{\prime}$-(1-(4-chlorophenyl)ethane-1,2-diyl)bis(2-nitrobenzenesulfonamide) (3m)<smiles>NS(=O)(=O)CC(NS(=O)(=O)[O-])c1ccc(Cl)cc1</smiles>

${ }^{1} \mathrm{H}$ NMR (400 MHz, DMSO- $\left.d_{6}\right)$

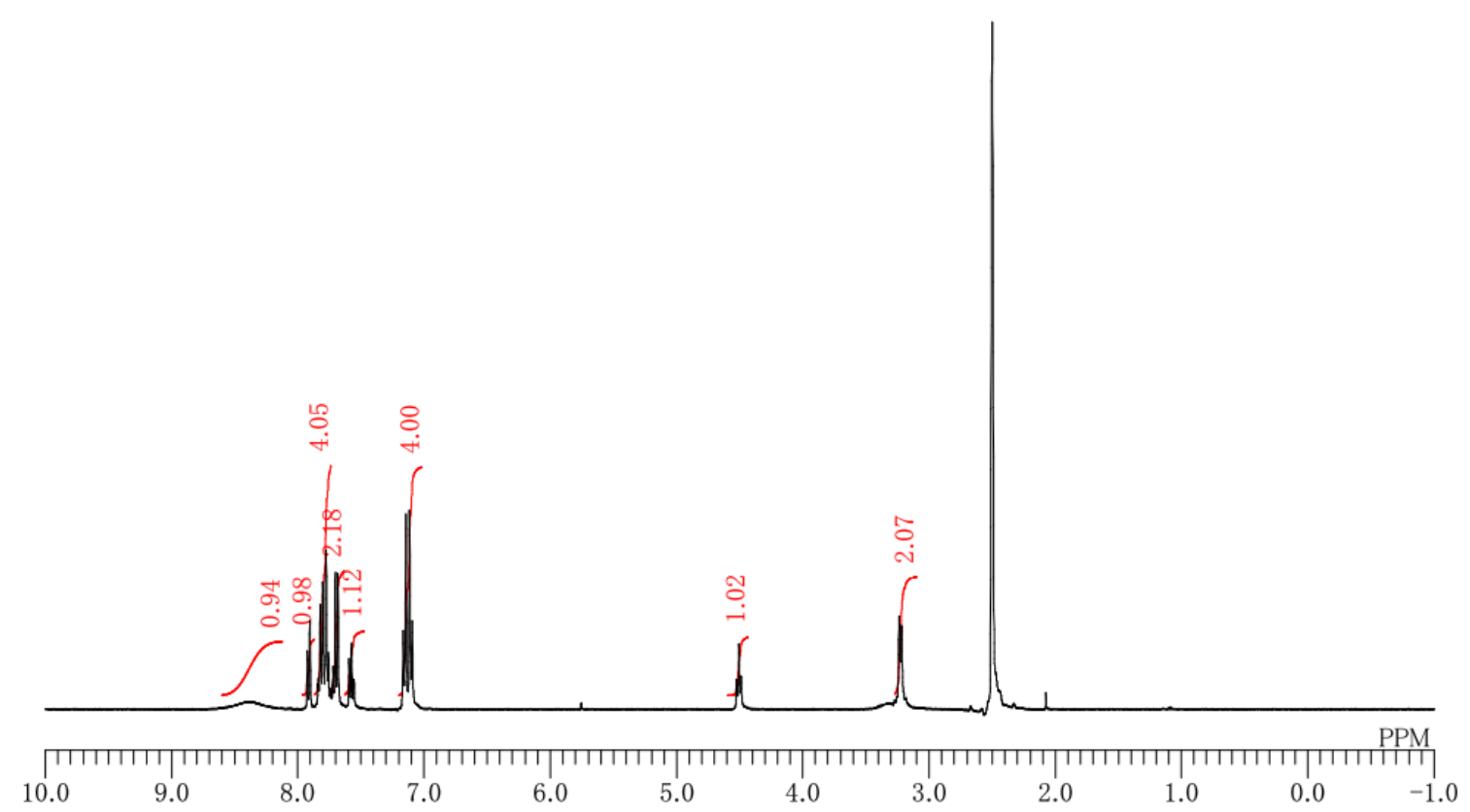

${ }^{13} \mathrm{C}$ NMR (100 MHz, DMSO- $\left.d_{6}\right)$
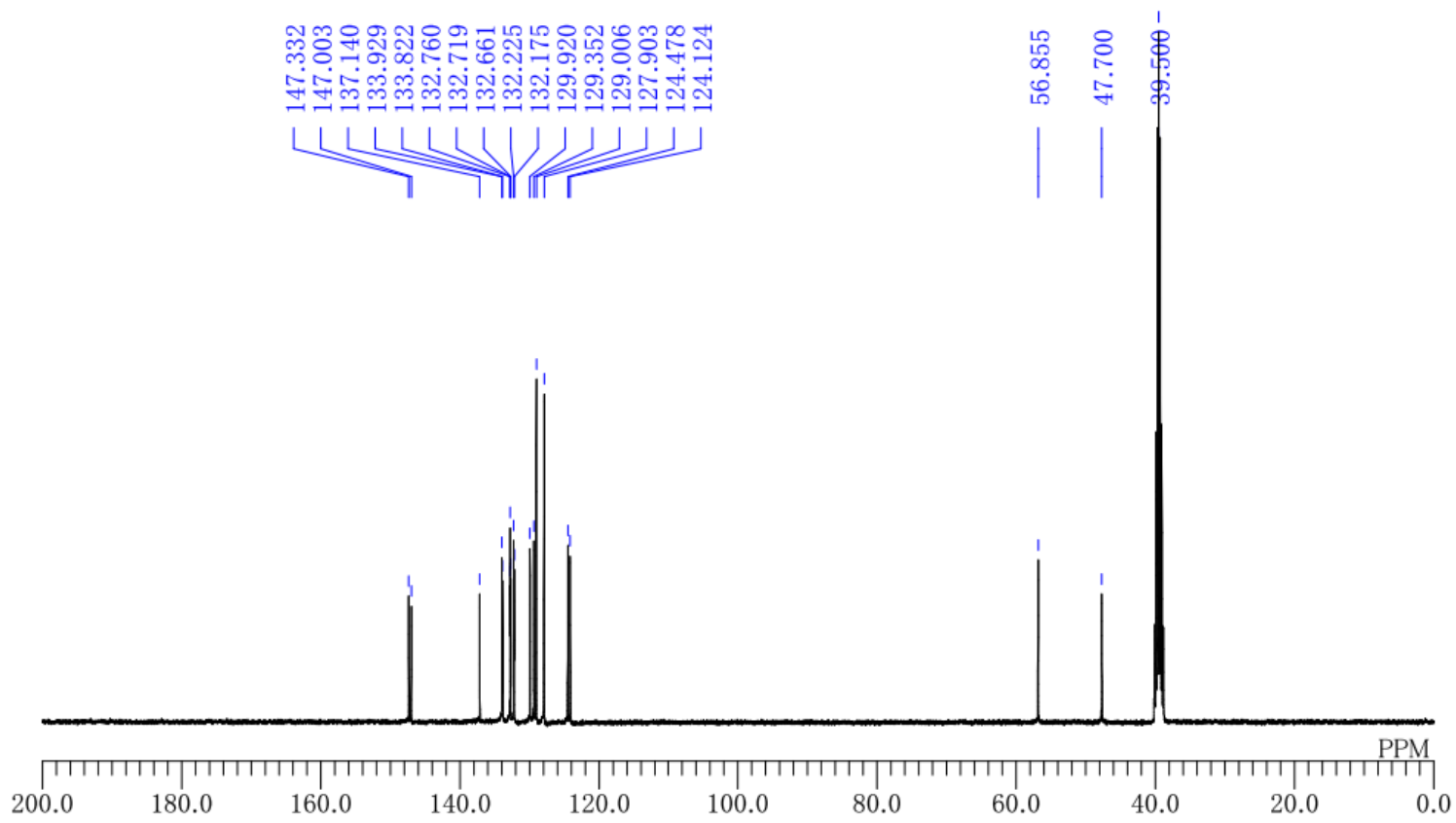
$N, N^{\prime}$-(1-(4-bromophenyl)ethane-1,2-diyl)bis(2-nitrobenzenesulfonamide) (3n)<smiles>O=S(=O)([O-])NC(CNS(=O)(=O)c1ccccc1)c1ccc(Br)cc1</smiles>

${ }^{1} \mathrm{H}$ NMR (400 MHz, DMSO- $d_{6}$ )

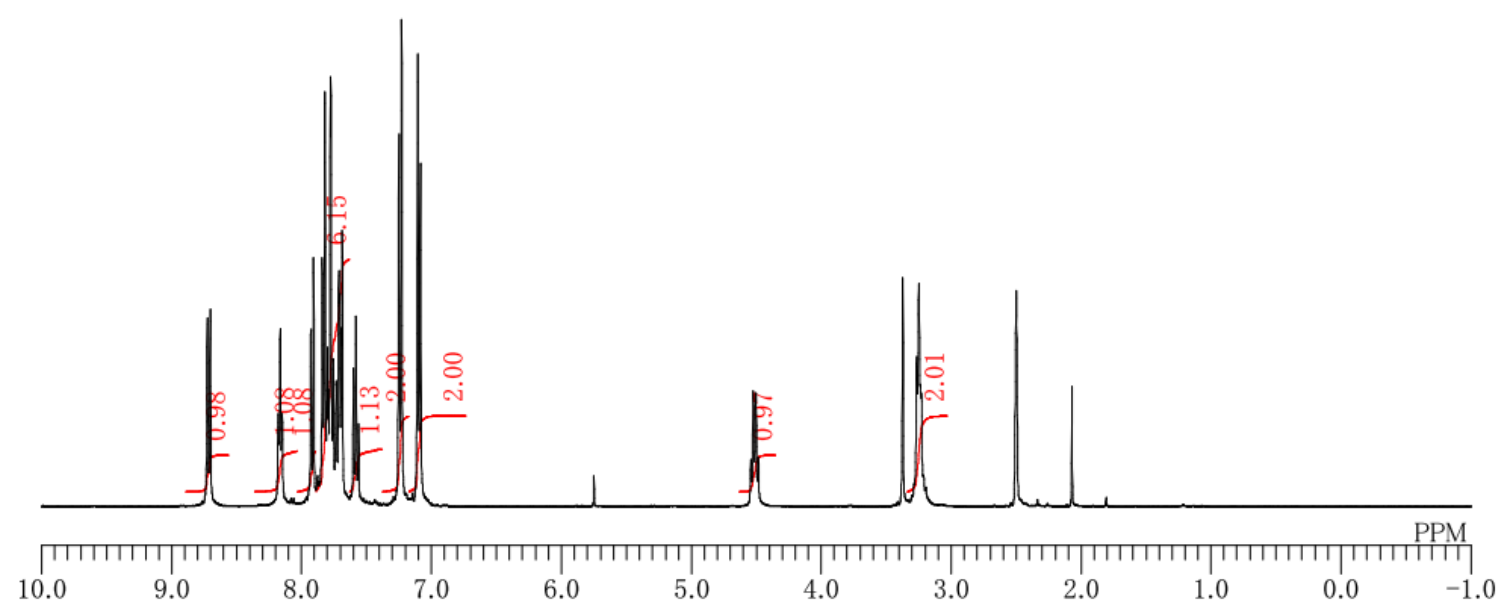

${ }^{13} \mathrm{C}$ NMR (100 MHz, DMSO- $\left.d_{6}\right)$
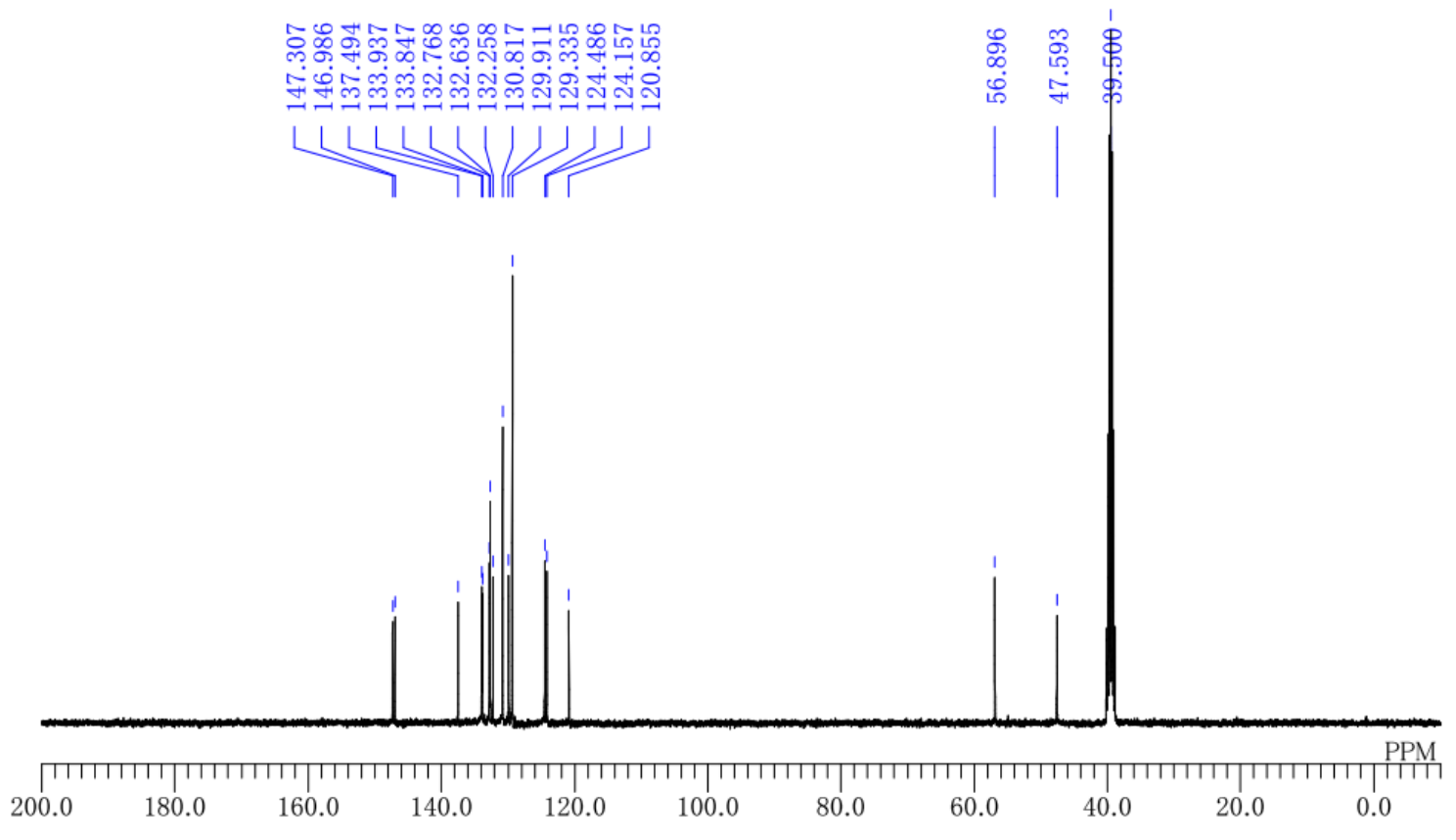
$N, N^{\prime}$-(1-(4-acetoxyphenyl)ethane-1,2-diyl)bis(2-nitrobenzenesulfonamide) (3o)<smiles>CC(=O)Oc1ccc(C(CNS(=O)(=O)[O-])NS(=O)(=O)[O-])cc1</smiles>

${ }^{1} \mathrm{H}$ NMR (400 MHz, DMSO- $\left.d_{6}\right)$

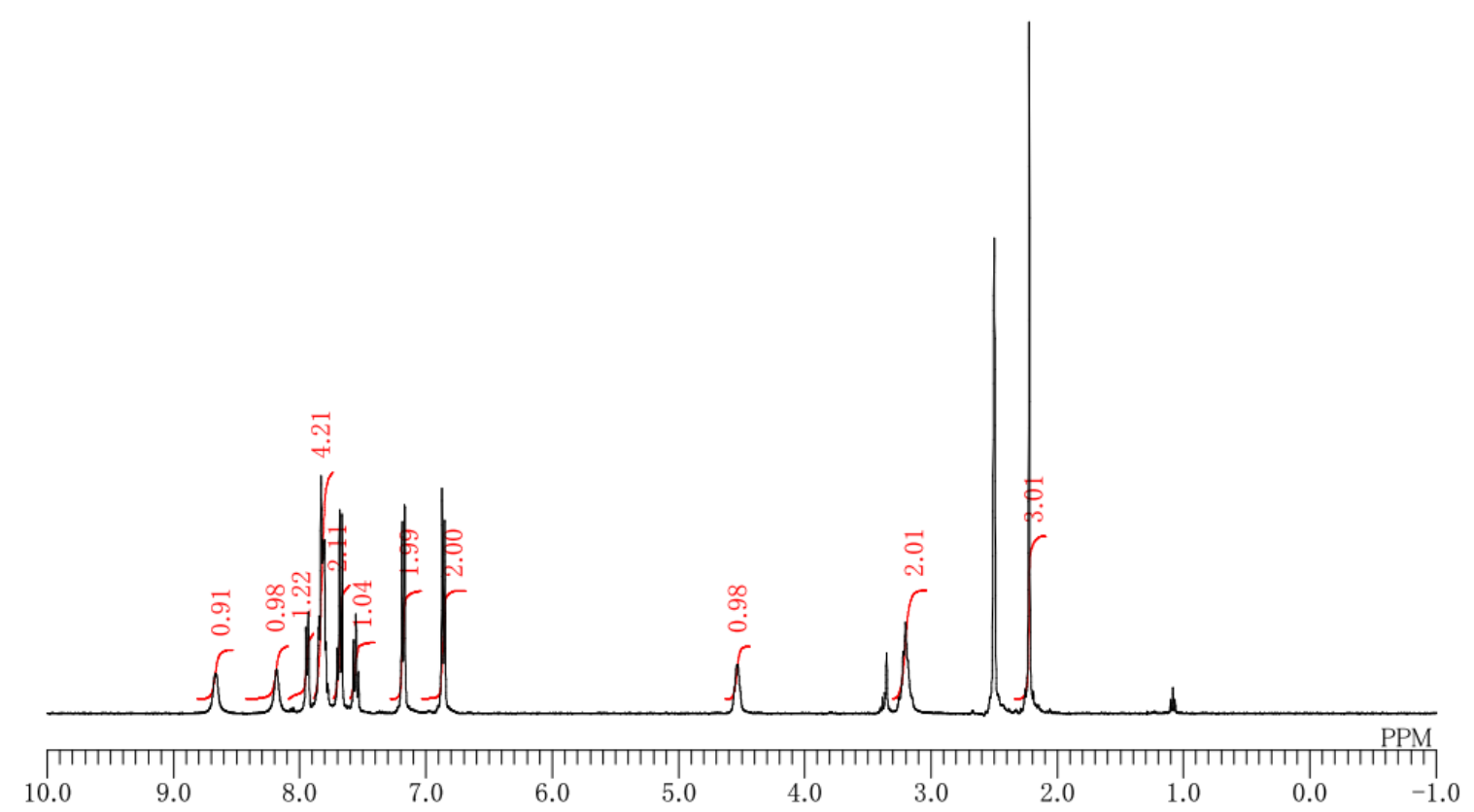

${ }^{13} \mathrm{C}$ NMR (100 MHz, DMSO- $\left.d_{6}\right)$
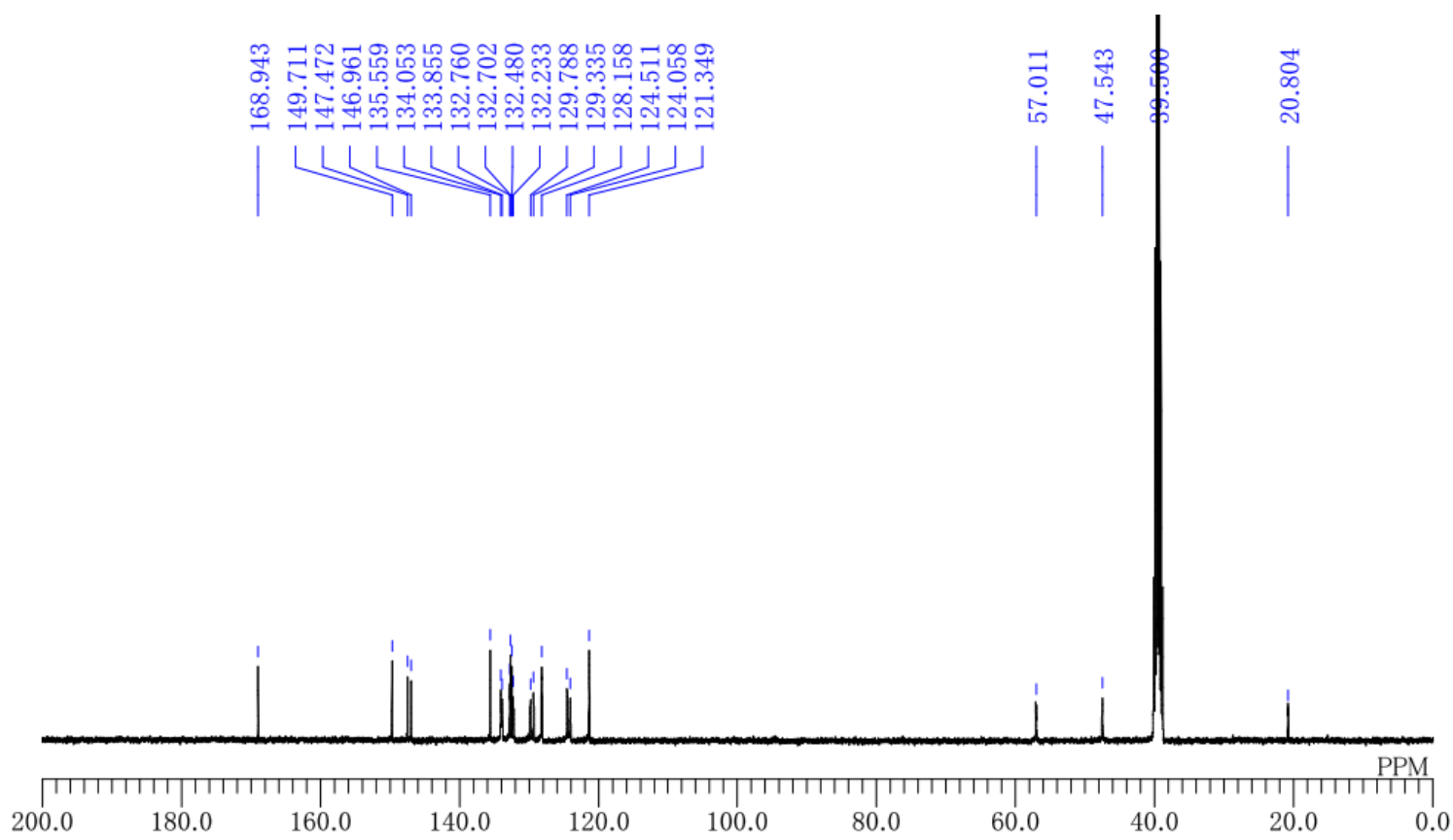
$N, N^{\prime}$-(1-(3-methoxyphenyl)ethane-1,2-diyl)bis(2-nitrobenzenesulfonamide) (3p)<smiles>COc1cccc(C(CNS(=O)(=O)[O-])C[N+](=O)[O-])c1</smiles>

${ }^{1} \mathrm{H}$ NMR (400 MHz, DMSO- $d_{6}$ )

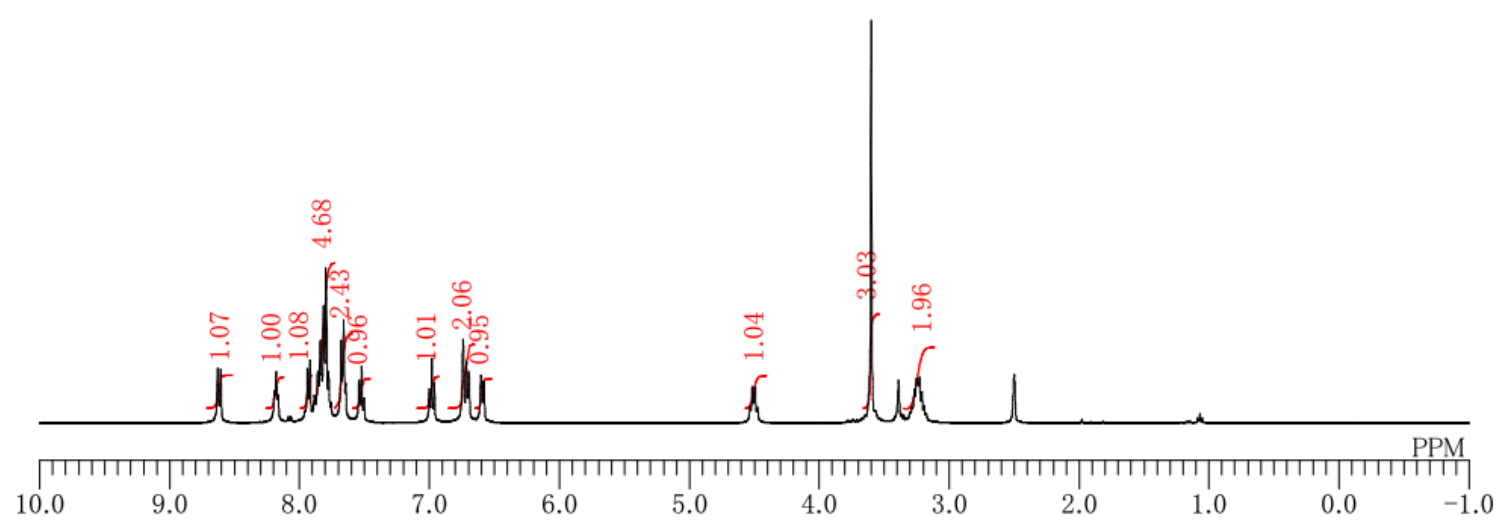

${ }^{13} \mathrm{C}$ NMR $\left(100 \mathrm{MHz}\right.$, DMSO- $\left.d_{6}\right)$
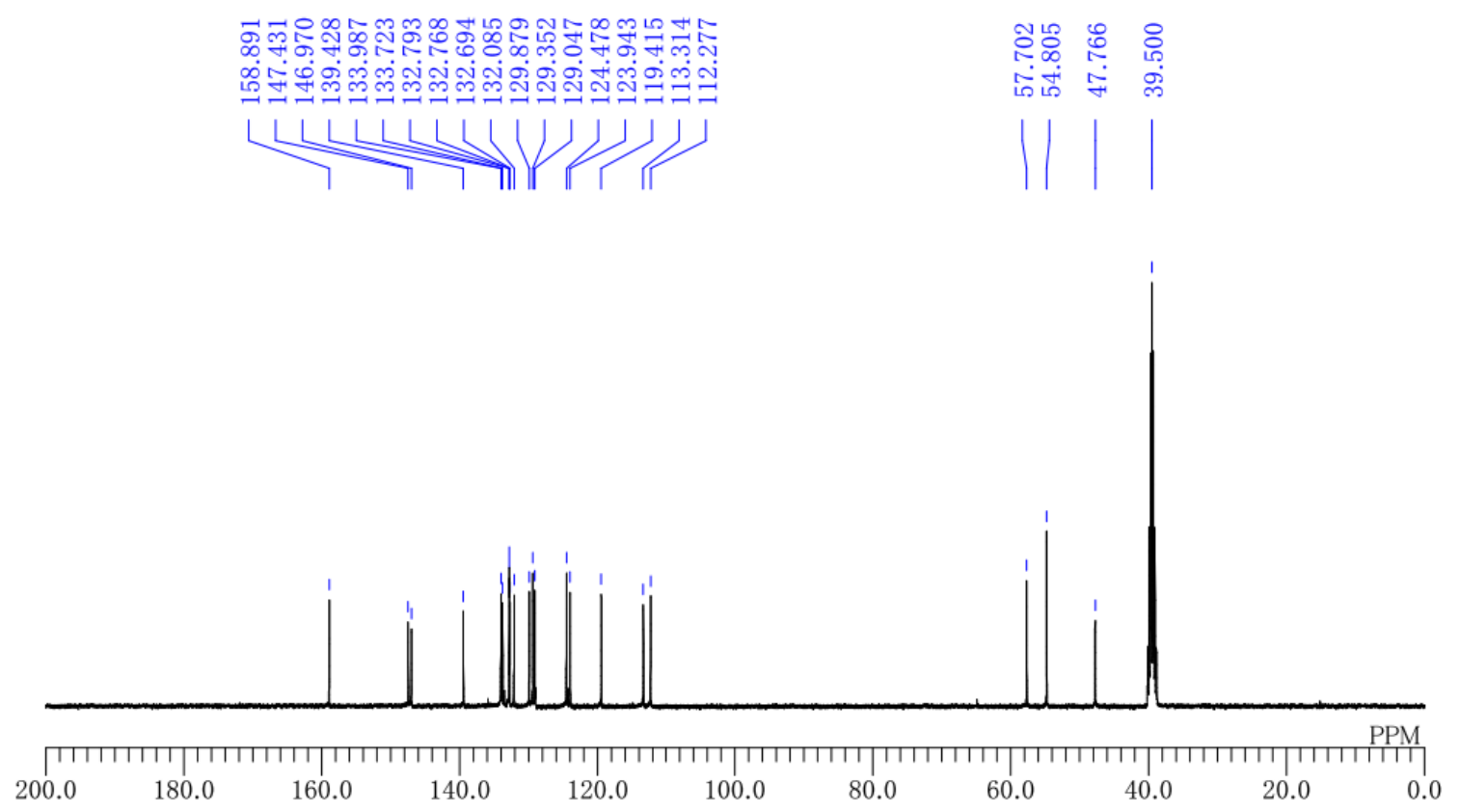
$N, N^{\prime}$-(1-(2-methylphenyl)ethane-1,2-diyl)bis(2-nitrobenzenesulfonamide) (3q)<smiles>Cc1ccccc1C(C[N+](=O)[O-])NS(=O)(=O)[O-]</smiles>

${ }^{1} \mathrm{H}$ NMR (400 MHz, DMSO- $\left.d_{6}\right)$

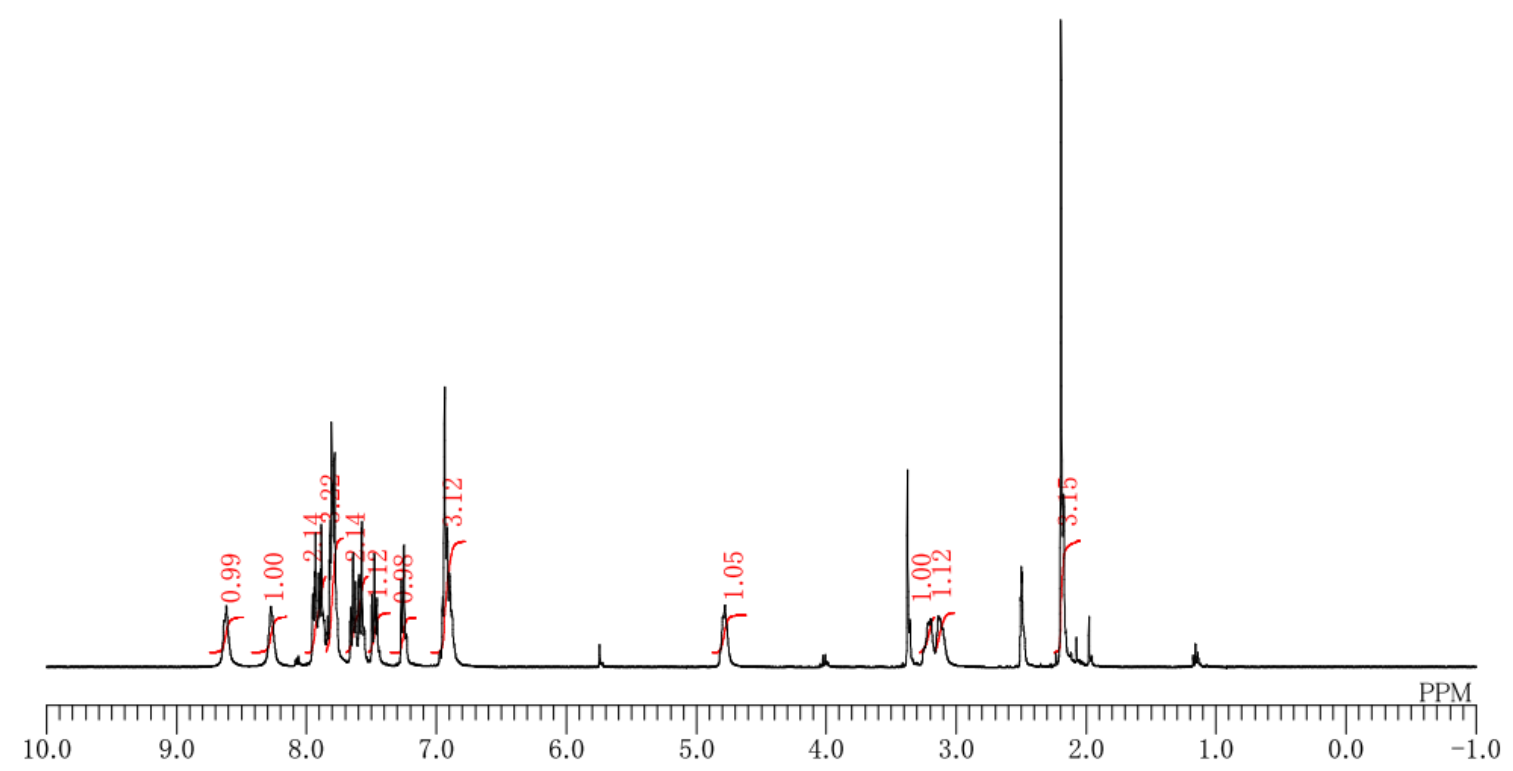

${ }^{13} \mathrm{C}$ NMR (100 MHz, DMSO- $\left.d_{6}\right)$

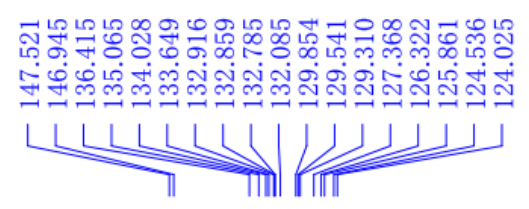

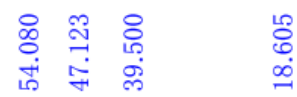
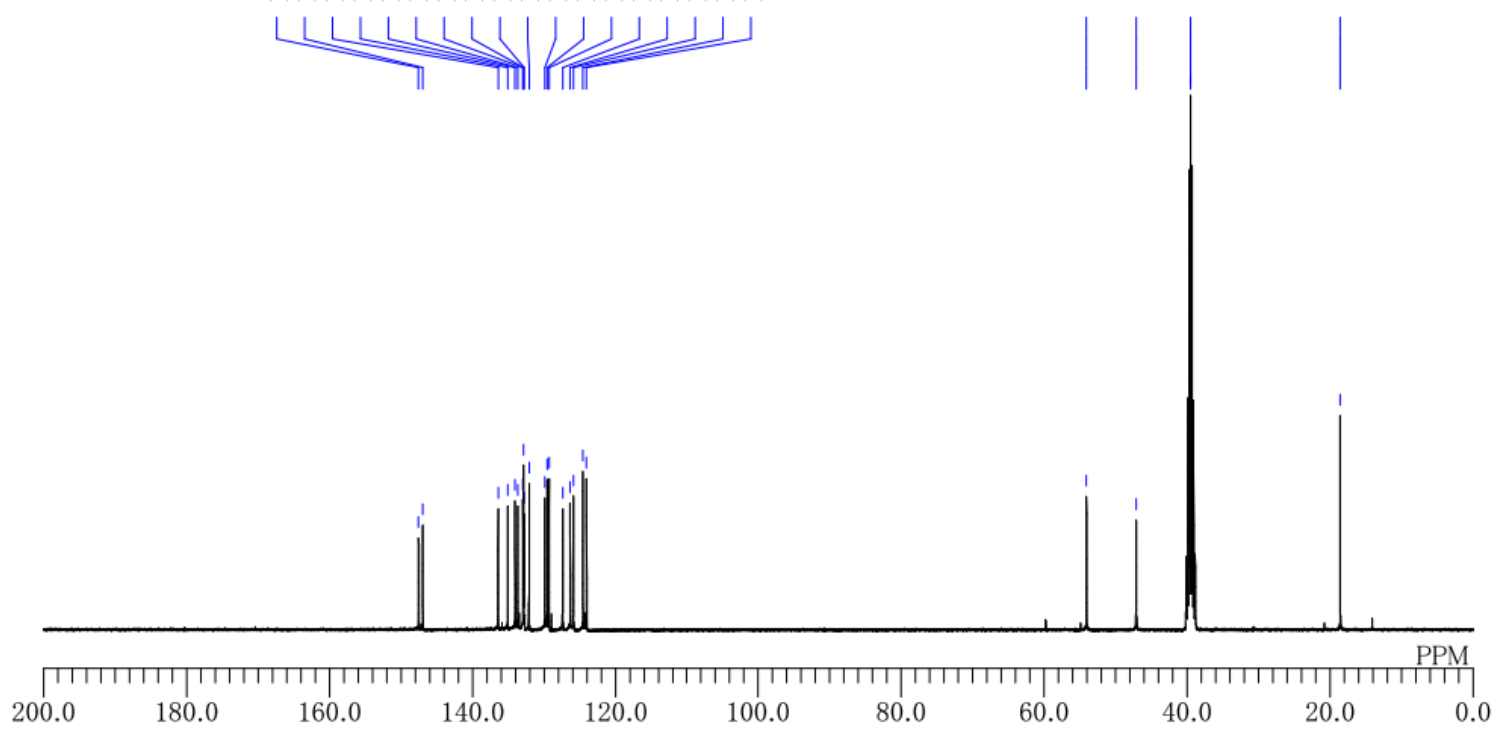
$N, N^{\prime}$-(1-(3-methylphenyl)ethane-1,2-diyl)bis(2-nitrobenzenesulfonamide) (3r)<smiles>Cc1cccc(C(CNS(=O)(=O)[O-])c2ccccc2)c1</smiles>

${ }^{1} \mathrm{H}$ NMR (400 MHz, DMSO-d $\left.{ }_{6}\right)$

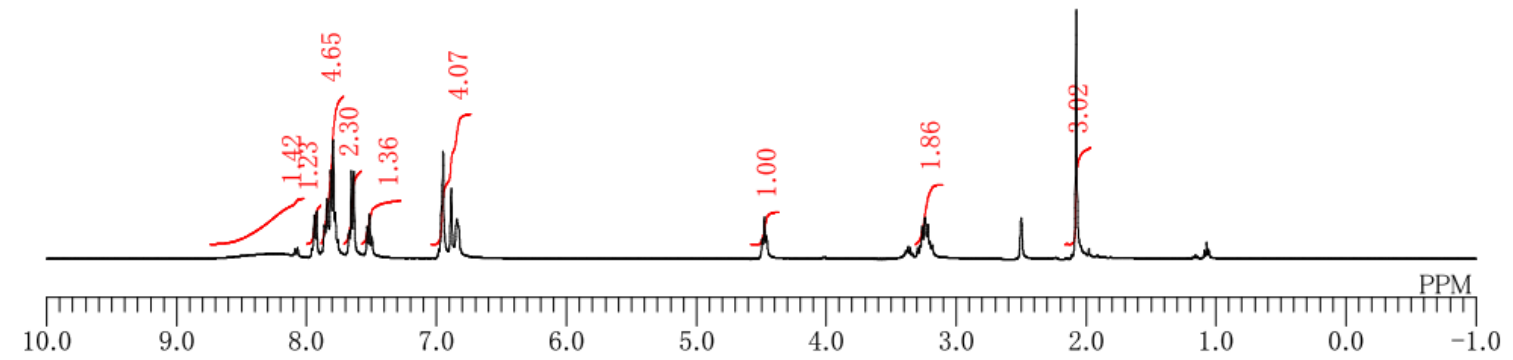

${ }^{13} \mathrm{C}$ NMR (100 MHz, DMSO- $d_{6}$ )
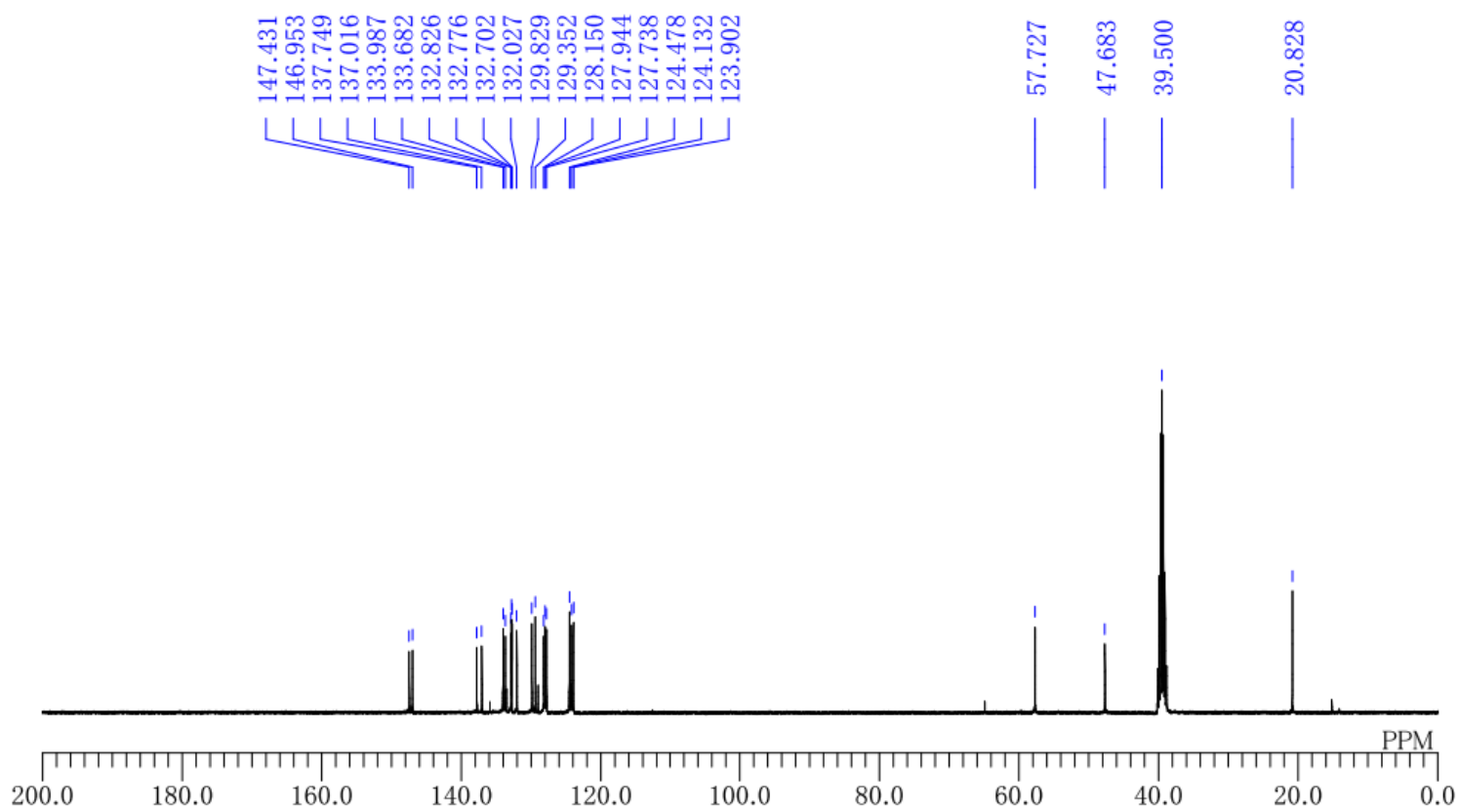
$N, N^{\prime}$-(1-(4-methylphenyl)ethane-1,2-diyl)bis(2-nitrobenzenesulfonamide) (3s)<smiles>Cc1ccc(C(CNS([N-])(=O)=O)NS(=O)(=O)[O-])cc1</smiles>

${ }^{1} \mathrm{H}$ NMR (400 MHz, DMSO- $\left.d_{6}\right)$

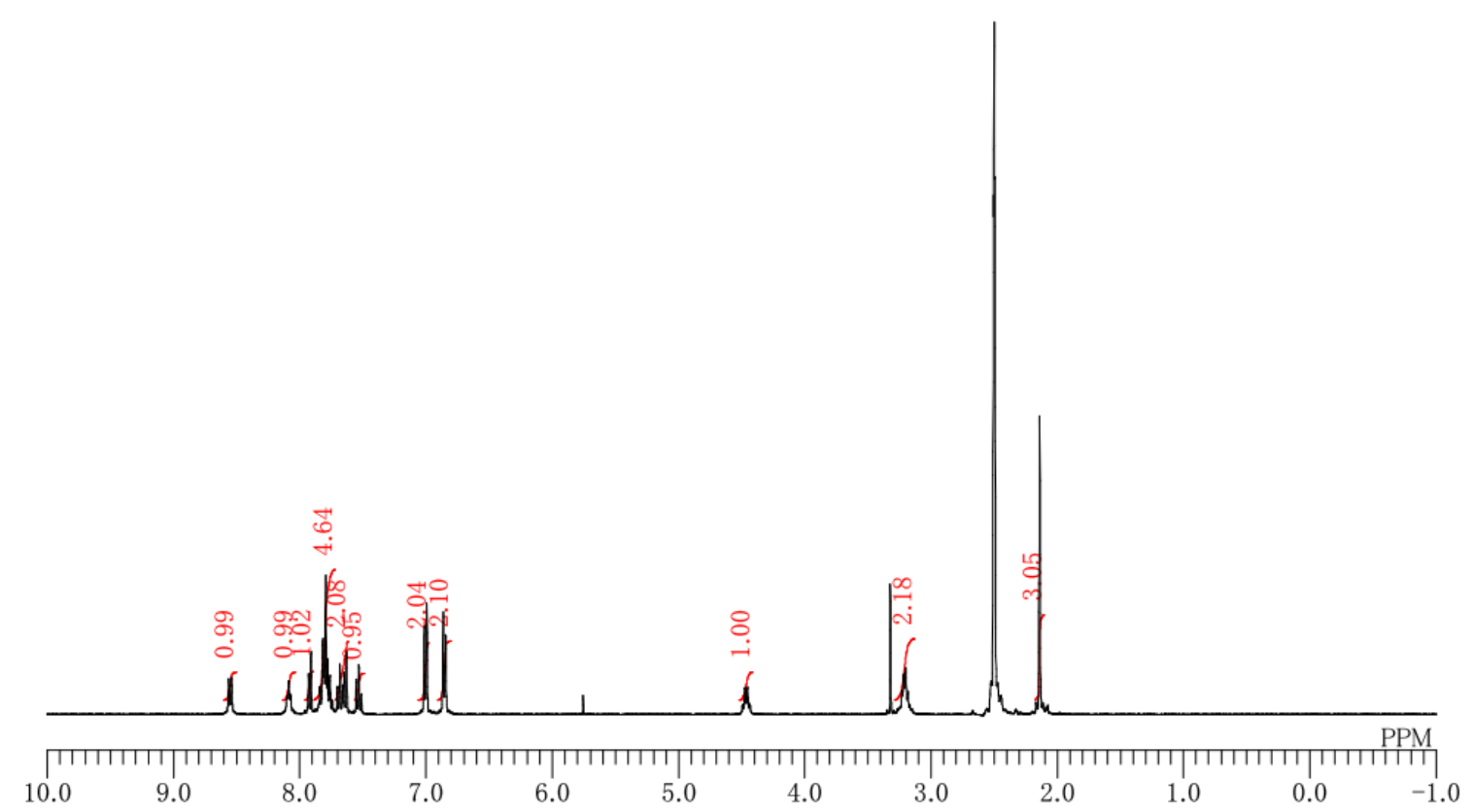

${ }^{13} \mathrm{C}$ NMR (100 MHz, DMSO- $\left.d_{6}\right)$
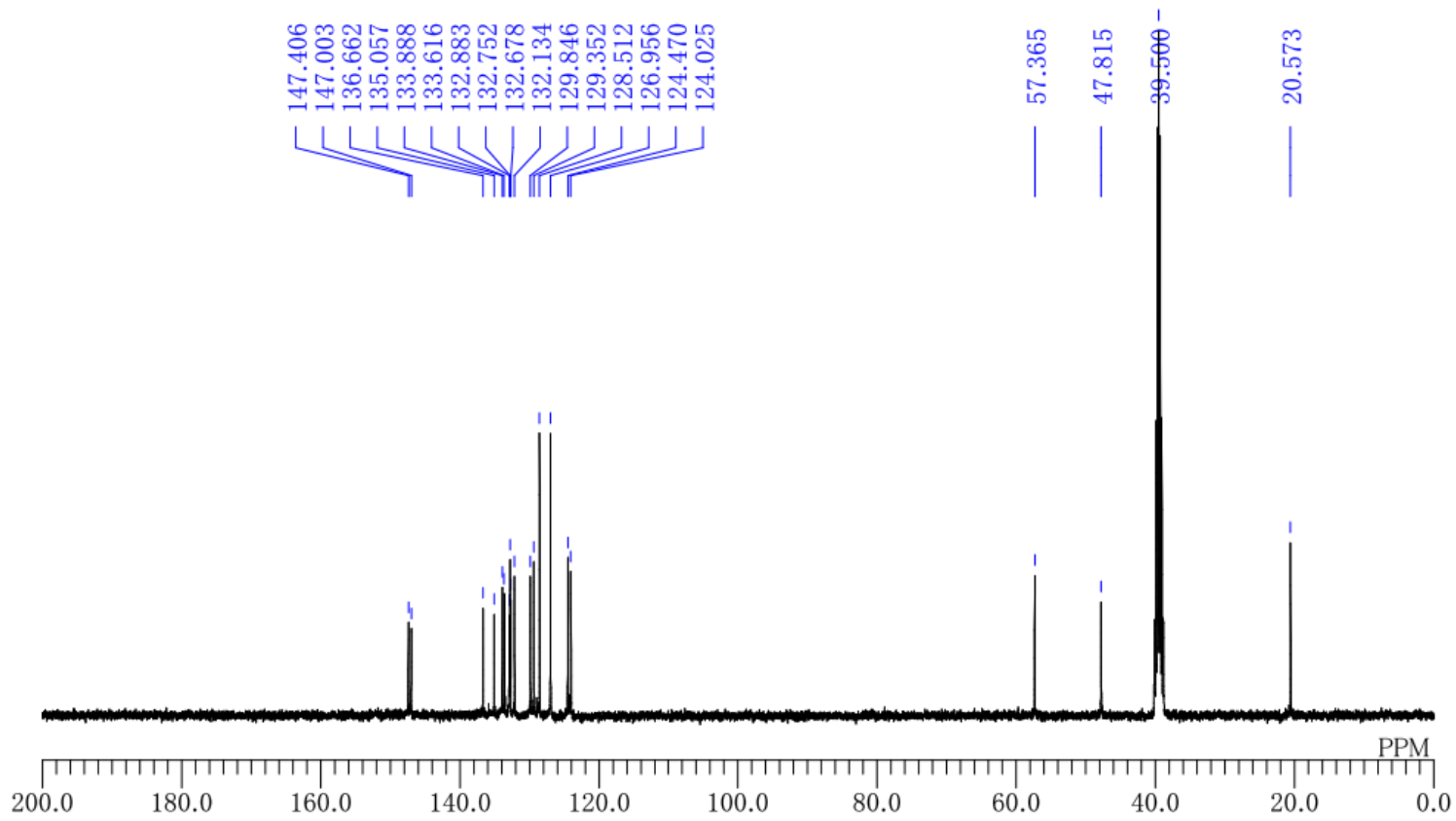
<smiles>CC(CNS([N-])(=O)=O)(c1ccccc1)c1ccccc1</smiles>

${ }^{1} \mathrm{H}$ NMR (400 MHz, acetone- $\left.d_{6}\right)$

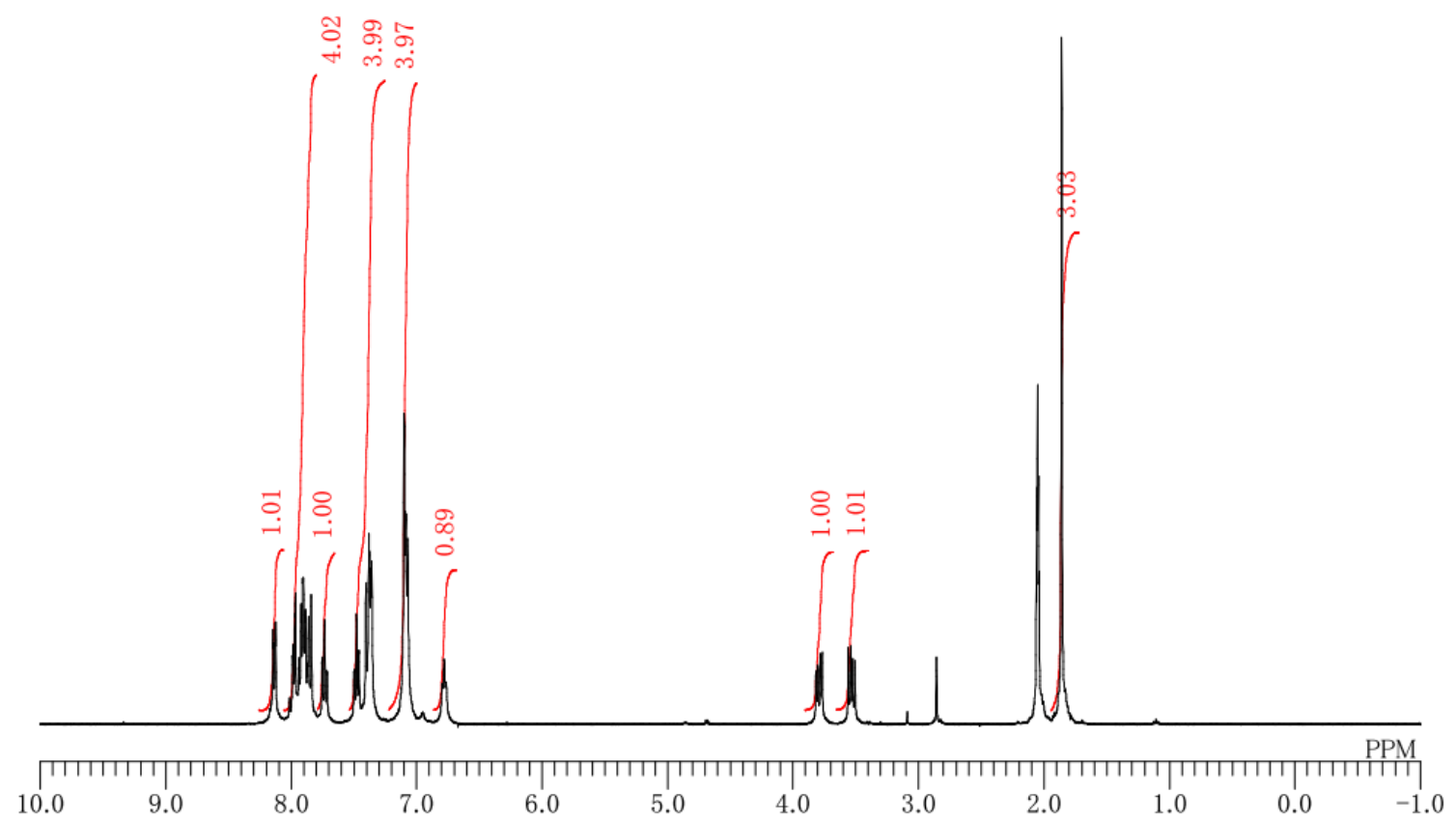

${ }^{13} \mathrm{C}$ NMR $\left(100 \mathrm{MHz}\right.$, acetone- $\left.d_{6}\right)$
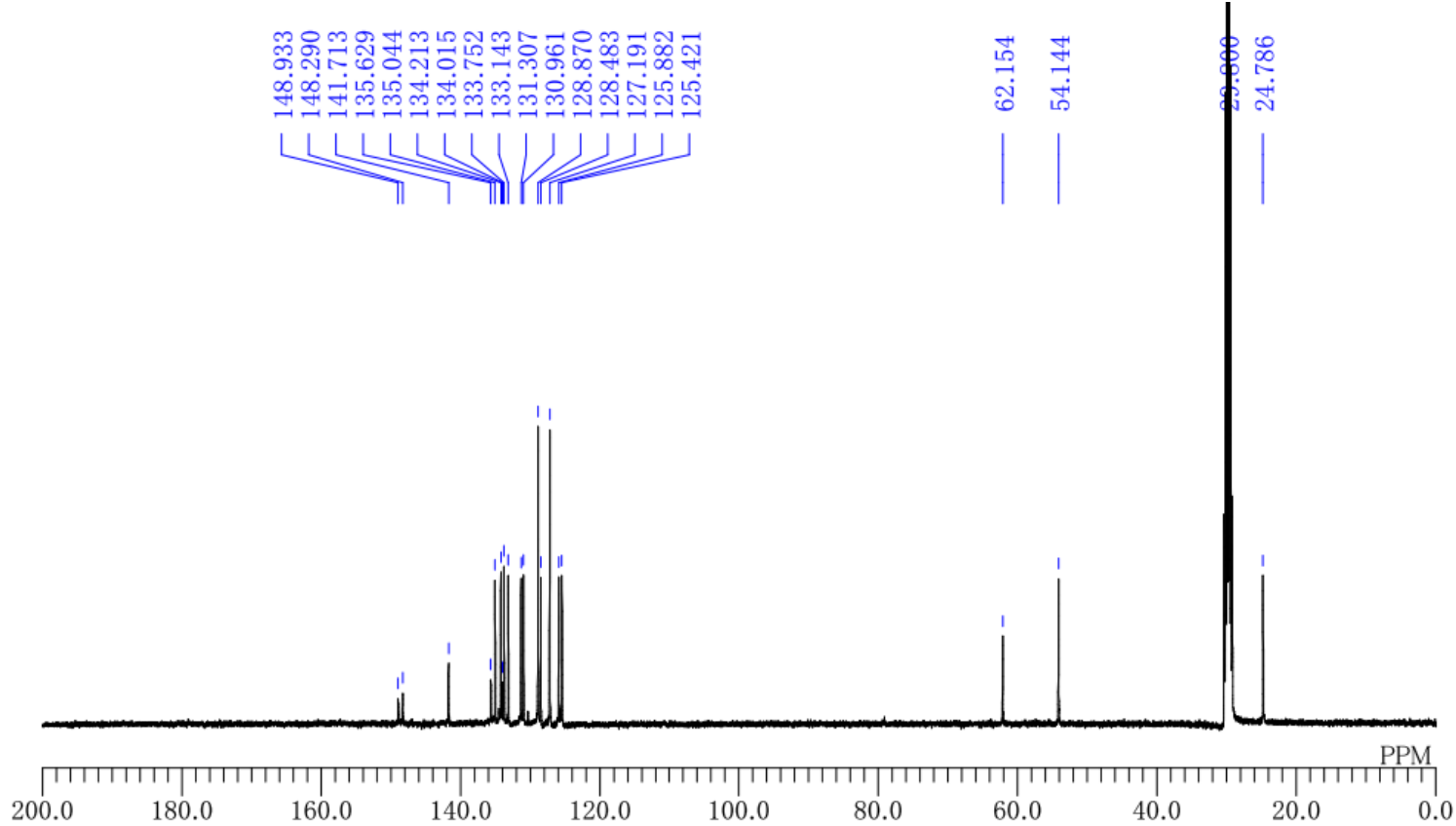
trans- $N, N^{\prime}$-(cyclopentane-1,2-diyl)bis(2-nitrobenzenesulfonamide) (4a)<smiles>N[C@H]1CCC[C@@H]1NS(N)(=O)=O</smiles>

${ }^{1} \mathrm{H}$ NMR (400 MHz, acetone- $\left.d_{6}\right)$

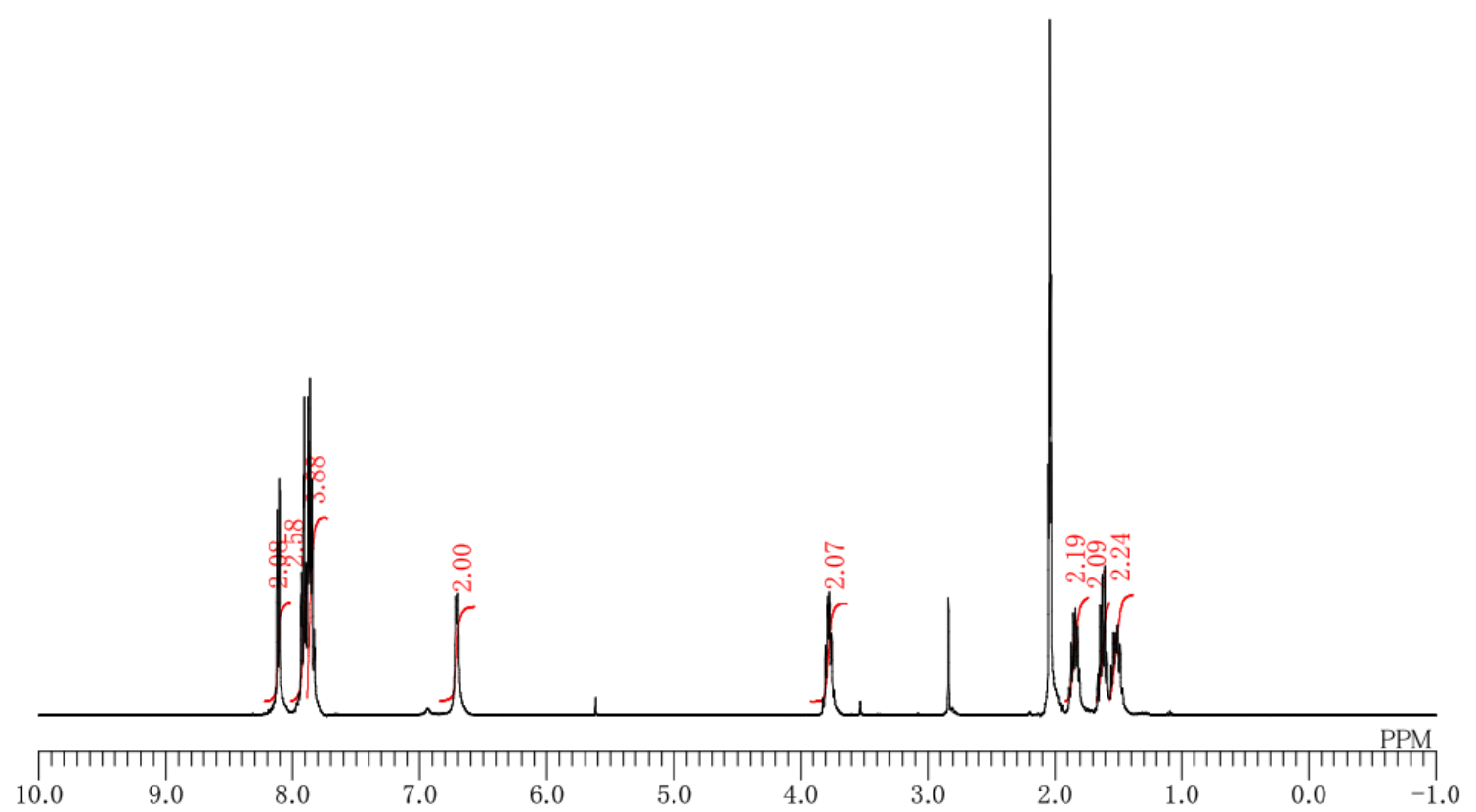

${ }^{13} \mathrm{C}$ NMR $\left(100 \mathrm{MHz}\right.$, acetone- $\left.d_{6}\right)$
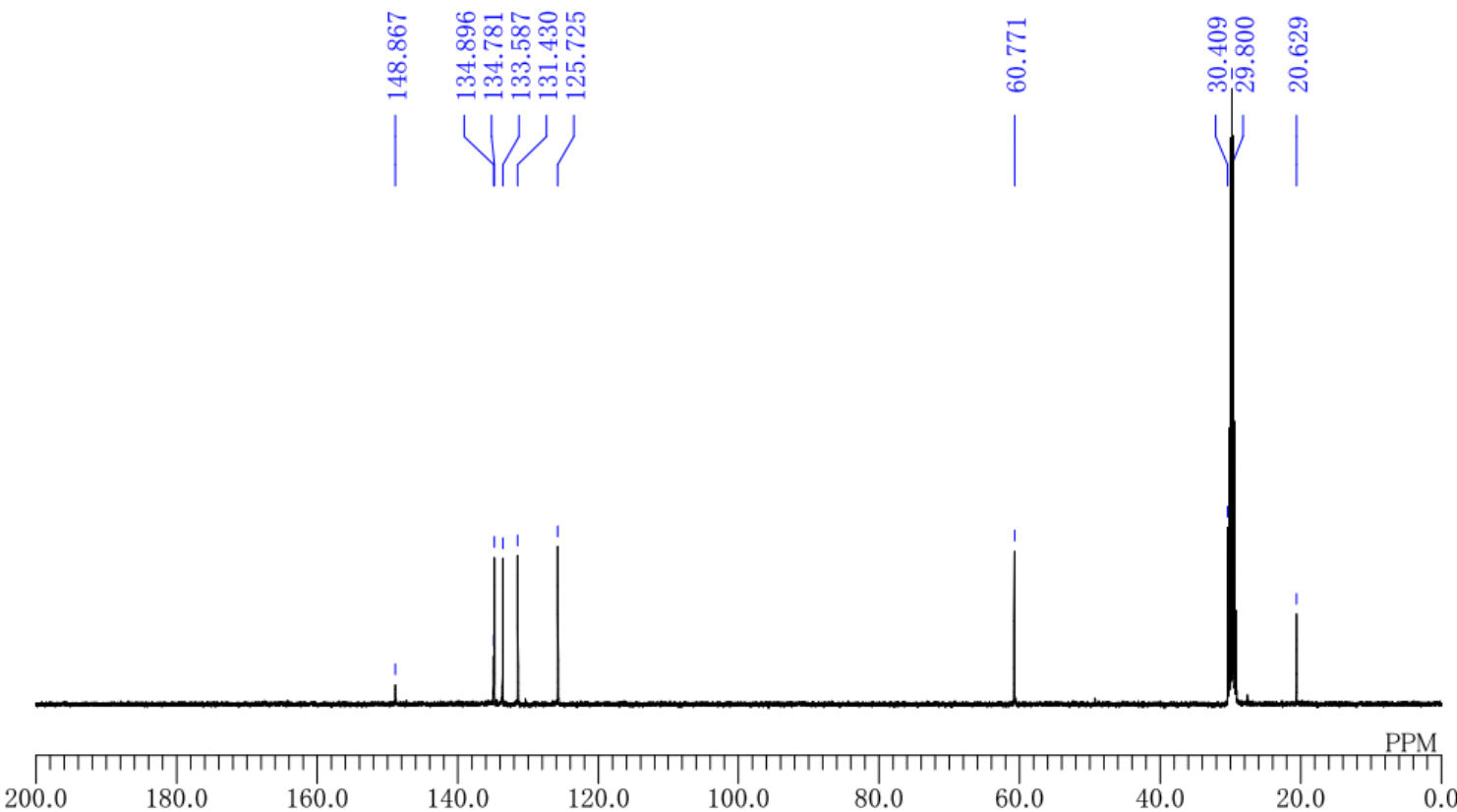
trans- $N, N^{\prime}$-(cyclohexane-1,2-diyl)bis(2-nitrobenzenesulfonamide) (4b)<smiles>[N+]S(=O)(=O)N[C@H]1CCCC[C@H]1N</smiles>

${ }^{1} \mathrm{H}$ NMR (400 MHz, DMSO- $\left.d_{6}\right)$

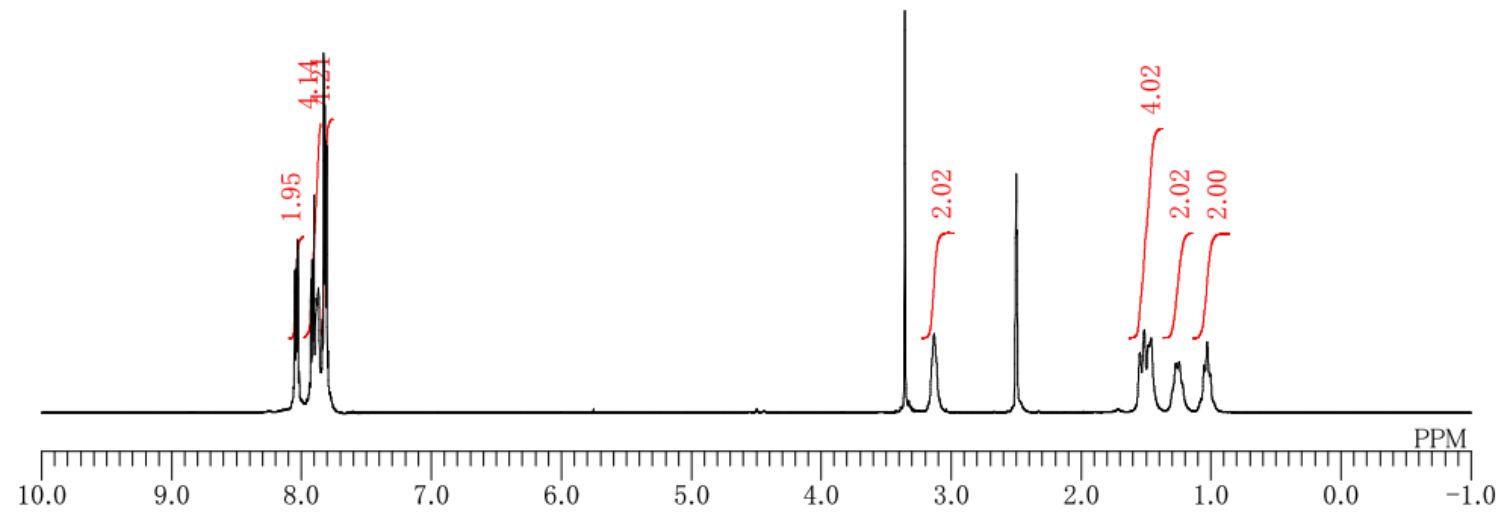

${ }^{13} \mathrm{C}$ NMR $\left(100 \mathrm{MHz}\right.$, DMSO- $\left.d_{6}\right)$
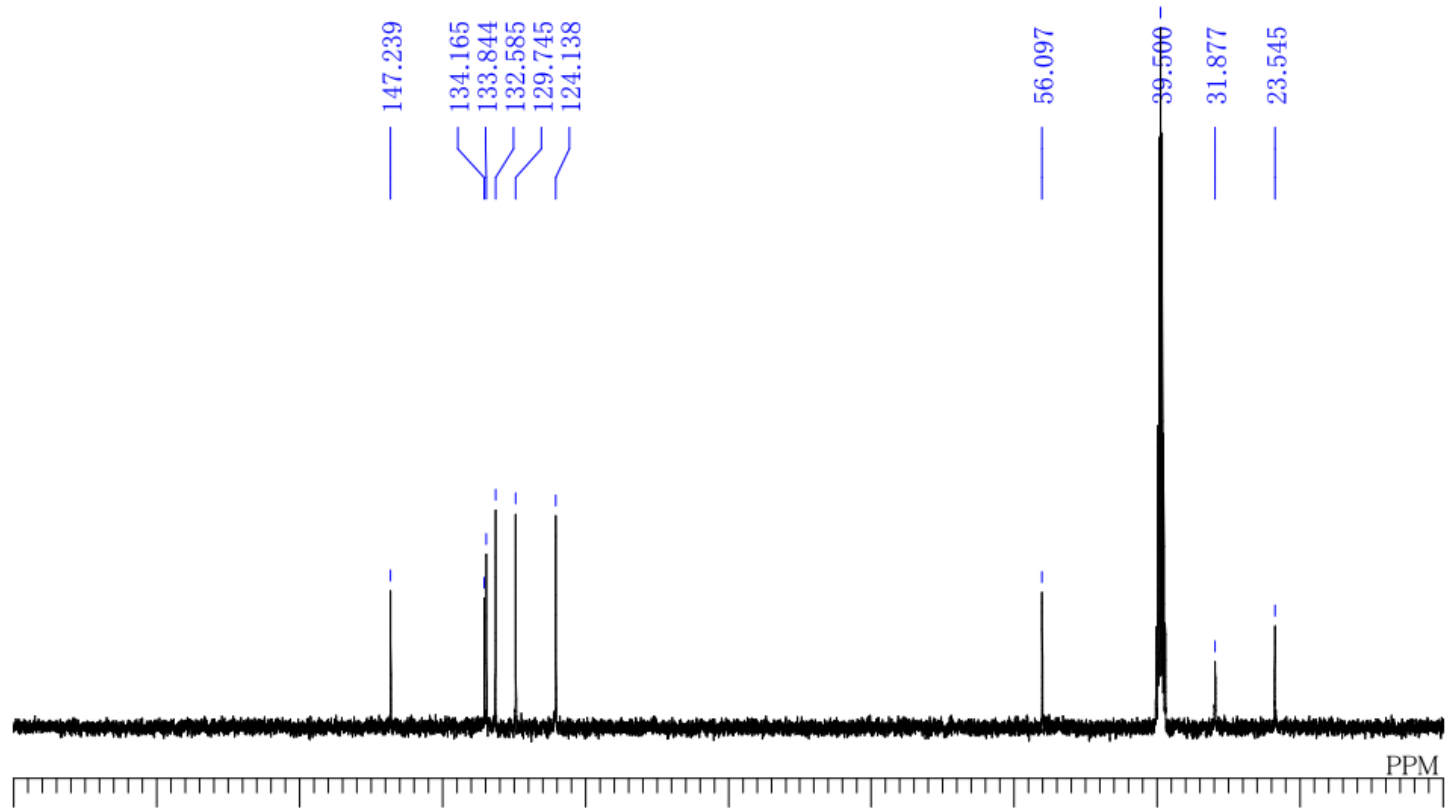

$\begin{array}{llllllllll}200.0 & 180.0 & 160.0 & 140.0 & 120.0 & 100.0 & 80.0 & 60.0 & 40.0 & 20.0\end{array}$ 
trans- $N, N^{\prime}$-(cyclohex-4-ene-1,2-diyl)bis(2-nitrobenzenesulfonamide) (4c)<smiles>NS(=O)(=O)[C@H]1CC=CC[C@H]1NS(=O)(=O)[O-]</smiles>

${ }^{1} \mathrm{H}$ NMR (400 MHz, DMSO- $\left.d_{6}\right)$

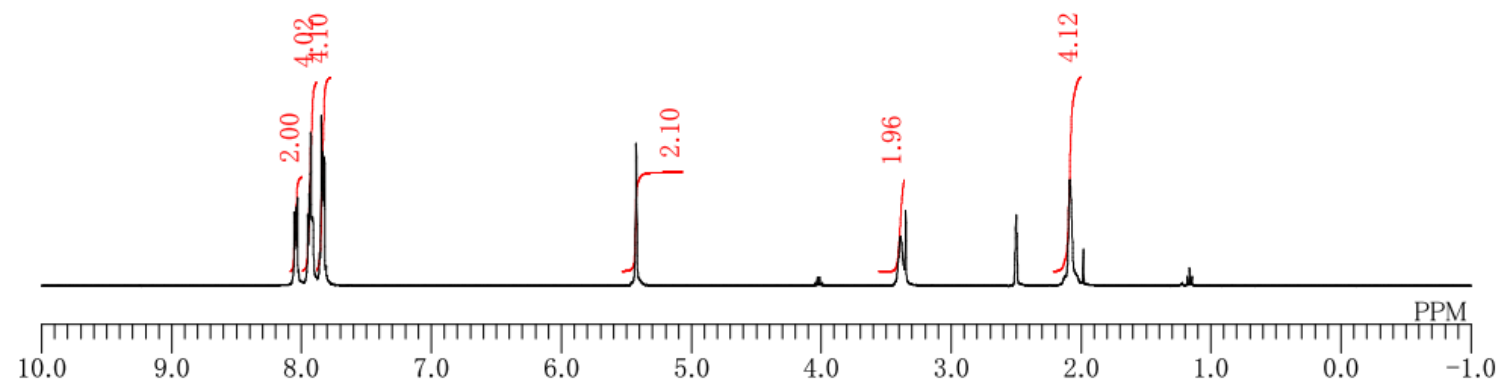

${ }^{13} \mathrm{C}$ NMR $\left(100 \mathrm{MHz}\right.$, DMSO- $\left.d_{6}\right)$

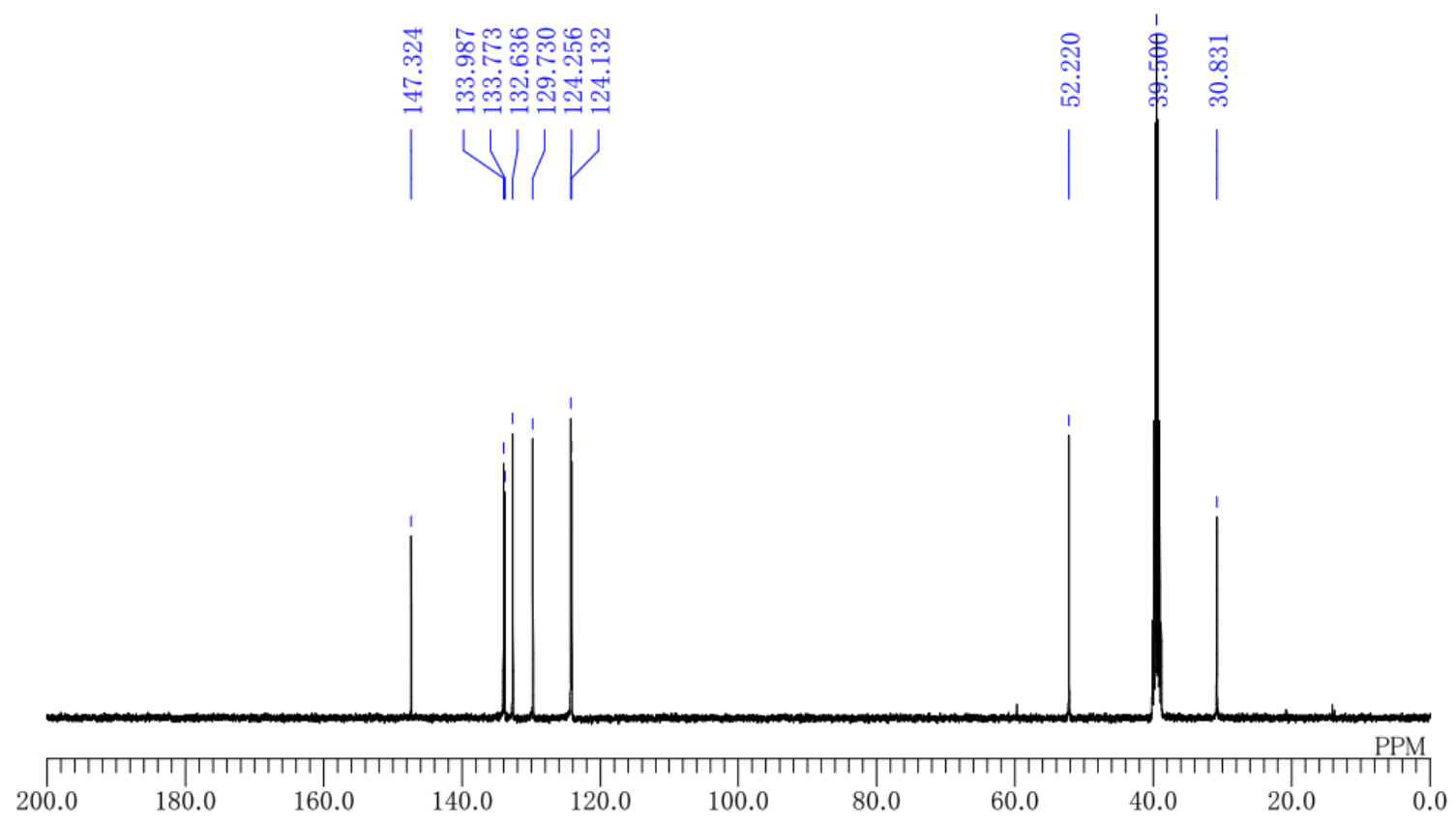


trans- $N, N^{\prime}$-(cyclohex-3-ene-1,2-diyl)bis(2-nitrobenzenesulfonamide) (4d)<smiles>NS(=O)(=O)N[C@H]1C=CCCC1[N+](=O)[O-]</smiles>

${ }^{1} \mathrm{H}$ NMR (400 MHz, DMSO- $\left.d_{6}\right)$

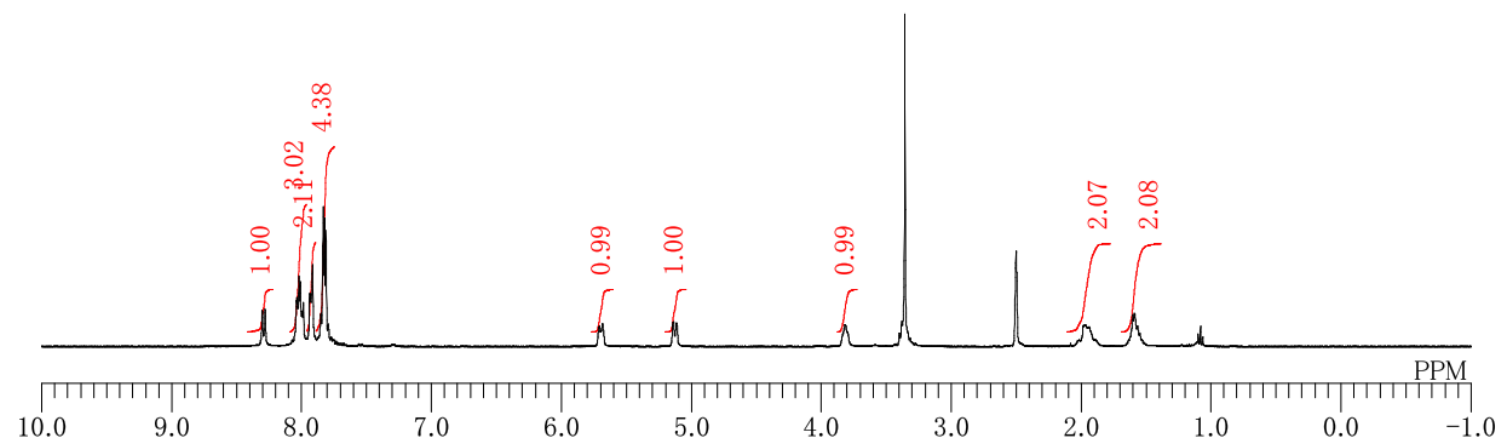

${ }^{13} \mathrm{C}$ NMR $\left(100 \mathrm{MHz}\right.$, DMSO- $\left.d_{6}\right)$
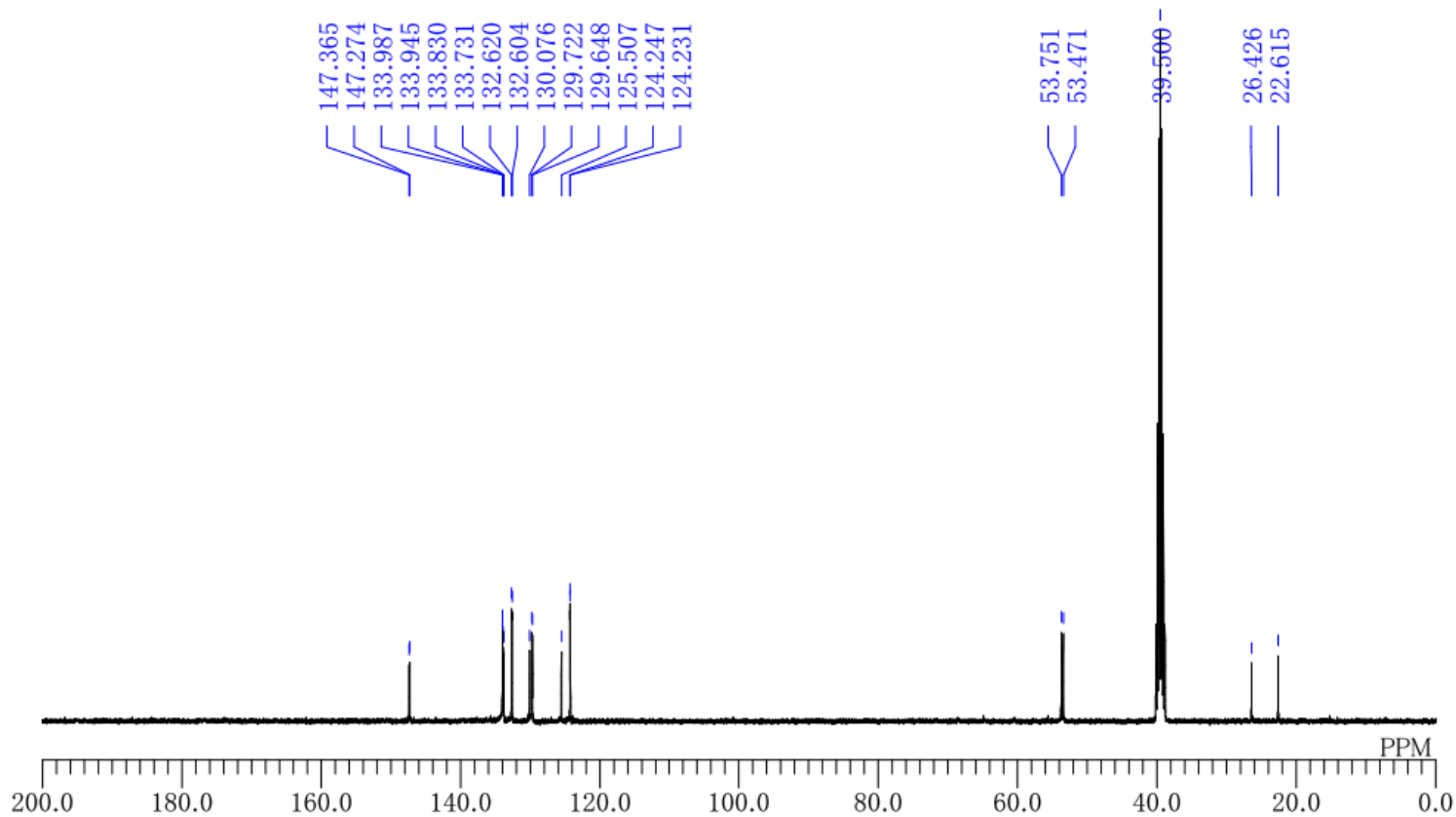
trans- $N, N^{\prime}$-(2,3-dihydro-1H-indene-1,2-diyl)bis(2-nitrobenzenesulfonamide) (4e)<smiles>NS(=O)(=O)[C@H]1c2ccccc2C[C@H]1NS(=O)(=O)[O-]</smiles>

${ }^{1} \mathrm{H}$ NMR (400 MHz, DMSO- $\left.d_{6}\right)$

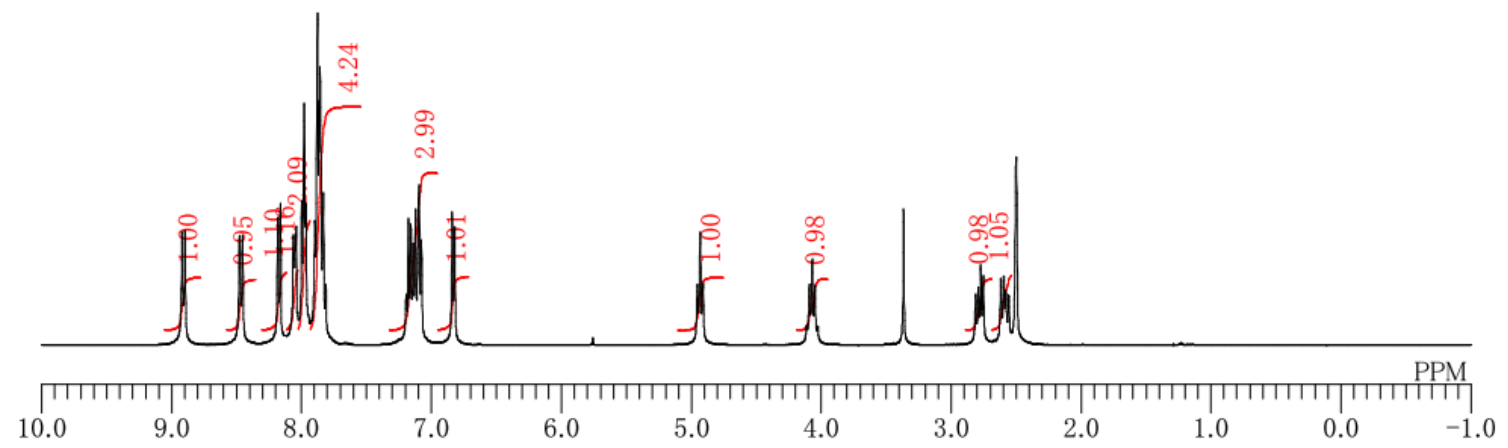

${ }^{13} \mathrm{C}$ NMR $\left(100 \mathrm{MHz}\right.$, DMSO- $\left.d_{6}\right)$
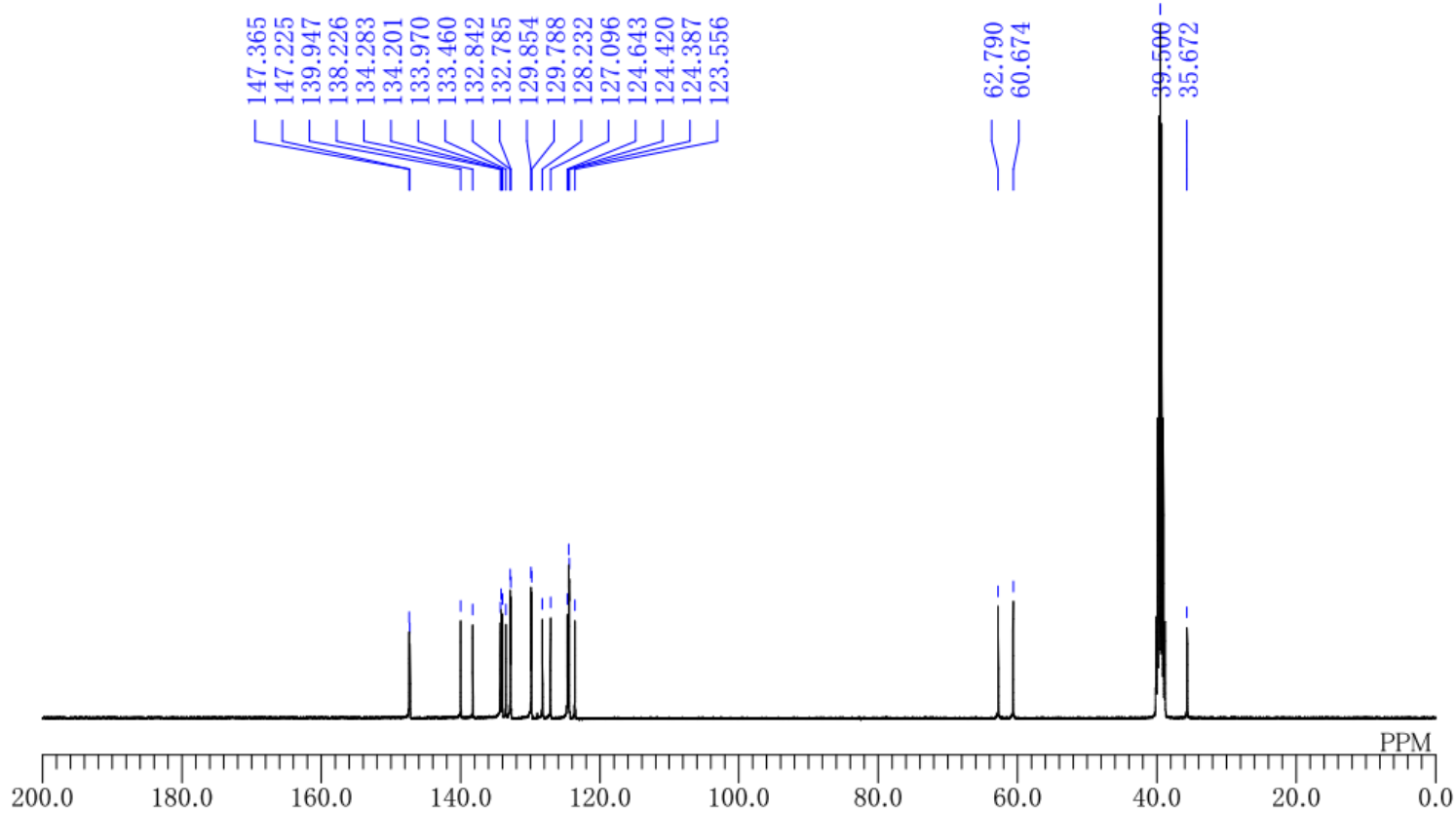
meso- $N, N^{\prime}$-(octane-4,5-diyl)bis(2-nitrobenzenesulfonamide) (4f)

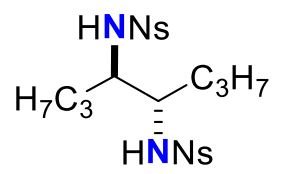

${ }^{1} \mathrm{H}$ NMR (400 MHz, $\left.\mathrm{CDCl}_{3}\right)$

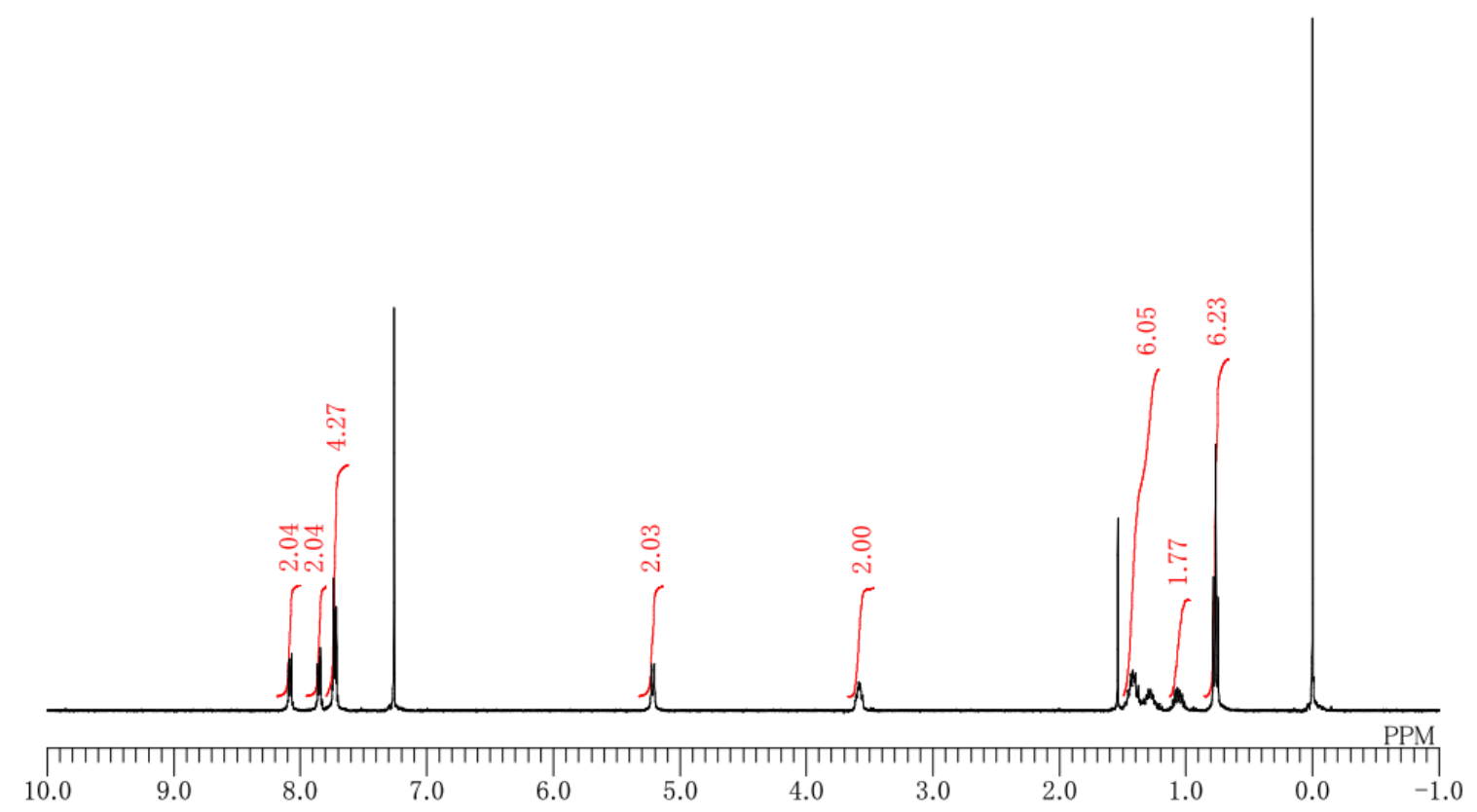

${ }^{13} \mathrm{C}$ NMR (100 MHz, $\mathrm{CDCl}_{3}$ )

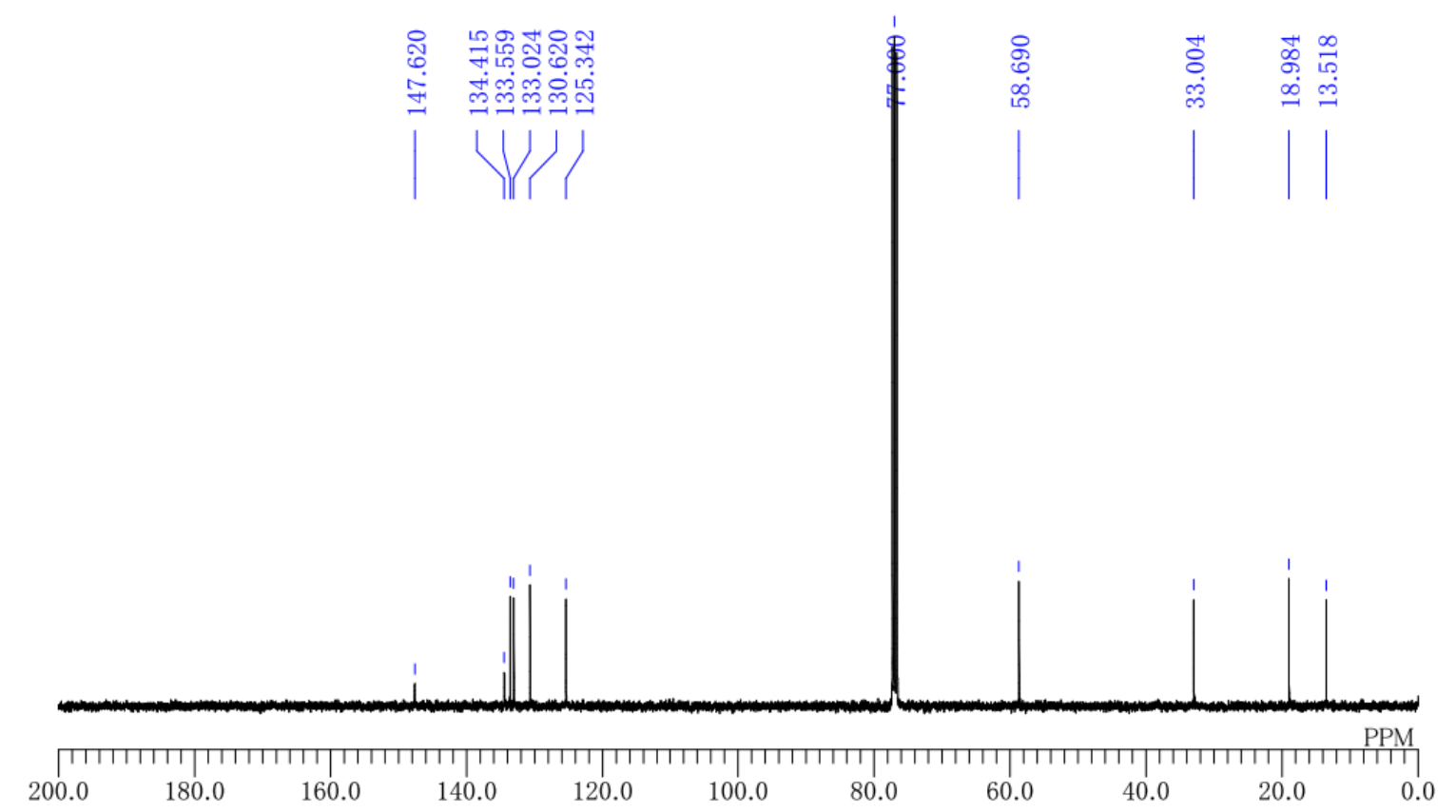


$N, N^{\prime}-\left(\left(4 R^{*}, 5 R^{*}\right)-\right.$-octane-4,5-diyl)bis(2-nitrobenzenesulfonamide) (4g)

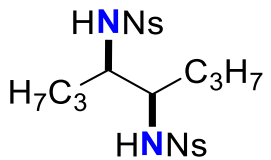

${ }^{1} \mathrm{H}$ NMR (400 MHz, $\left.\mathrm{CDCl}_{3}\right)$

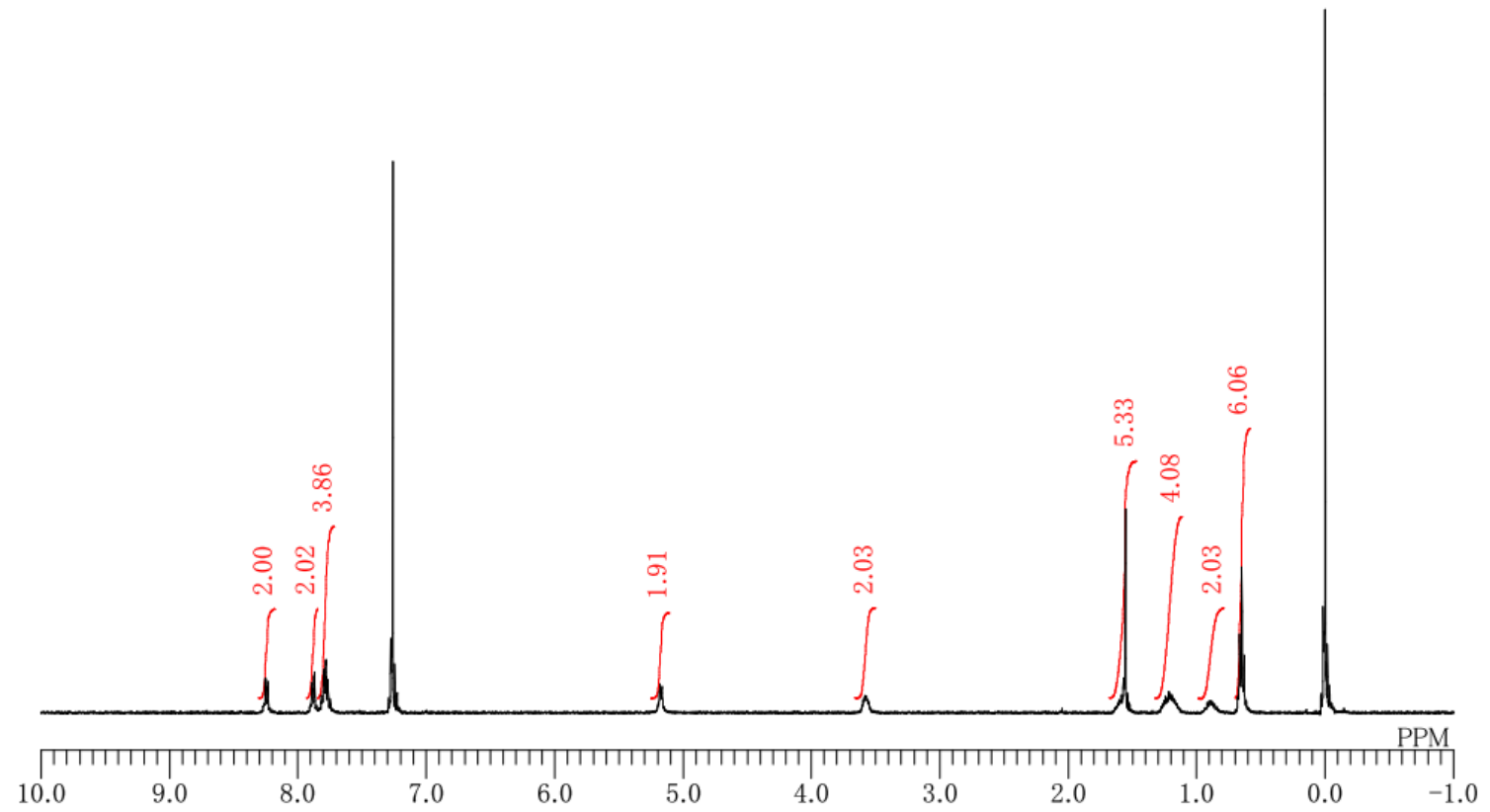

${ }^{13} \mathrm{C}$ NMR $\left(100 \mathrm{MHz}, \mathrm{CDCl}_{3}\right)$
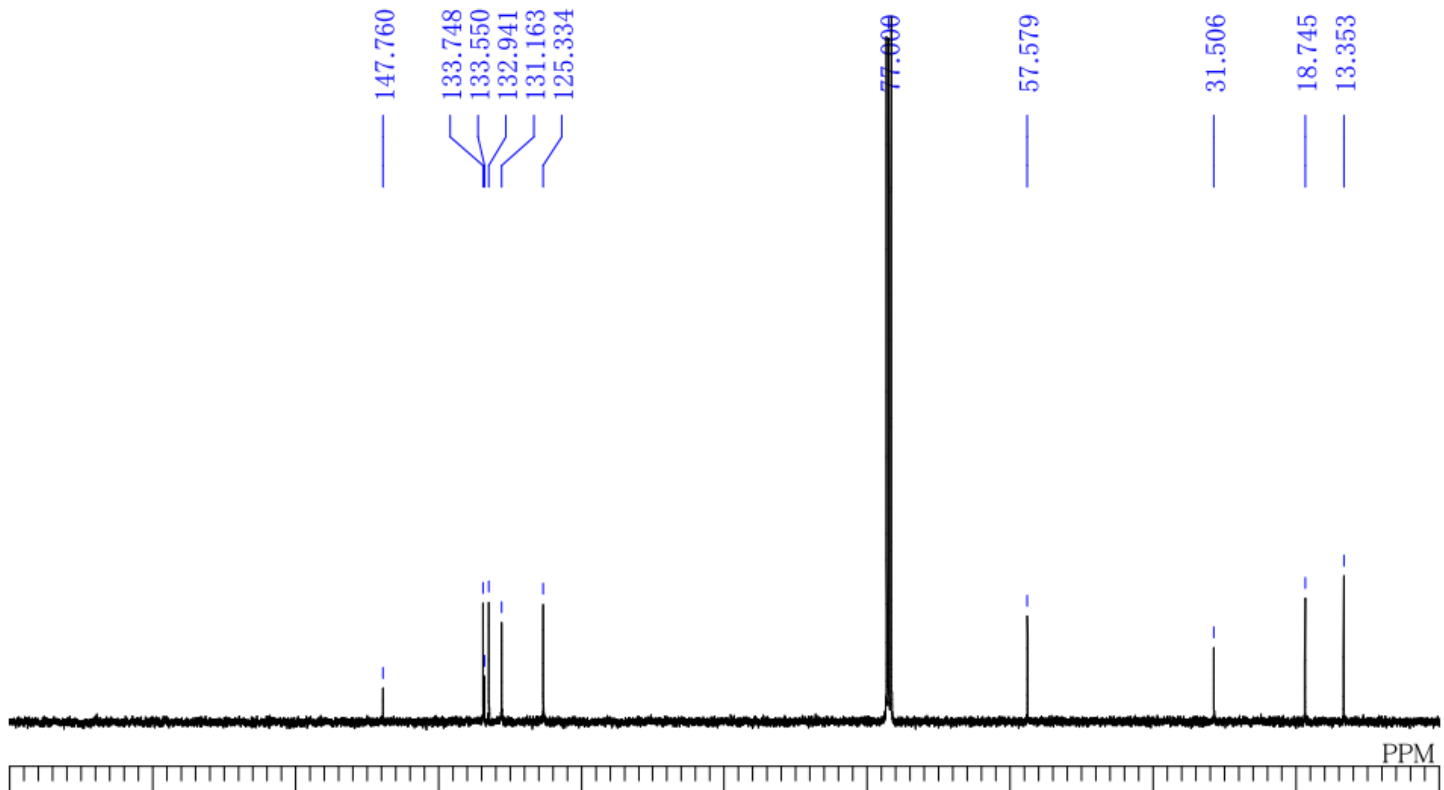

$\begin{array}{llllllllll}200.0 & 180.0 & 160.0 & 140.0 & 120.0 & 100.0 & 80.0 & 60.0 & 40.0 & 20.0\end{array}$ 
$N, N^{\prime}$-((2 $\left.R^{*}, 3 S^{*}\right)$-octane-2,3-diyl)bis(2-nitrobenzenesulfonamide) (4h)

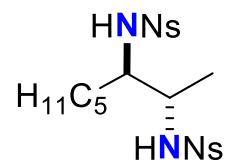

${ }^{1} \mathrm{H}$ NMR (400 MHz, $\left.\mathrm{CDCl}_{3}\right)$

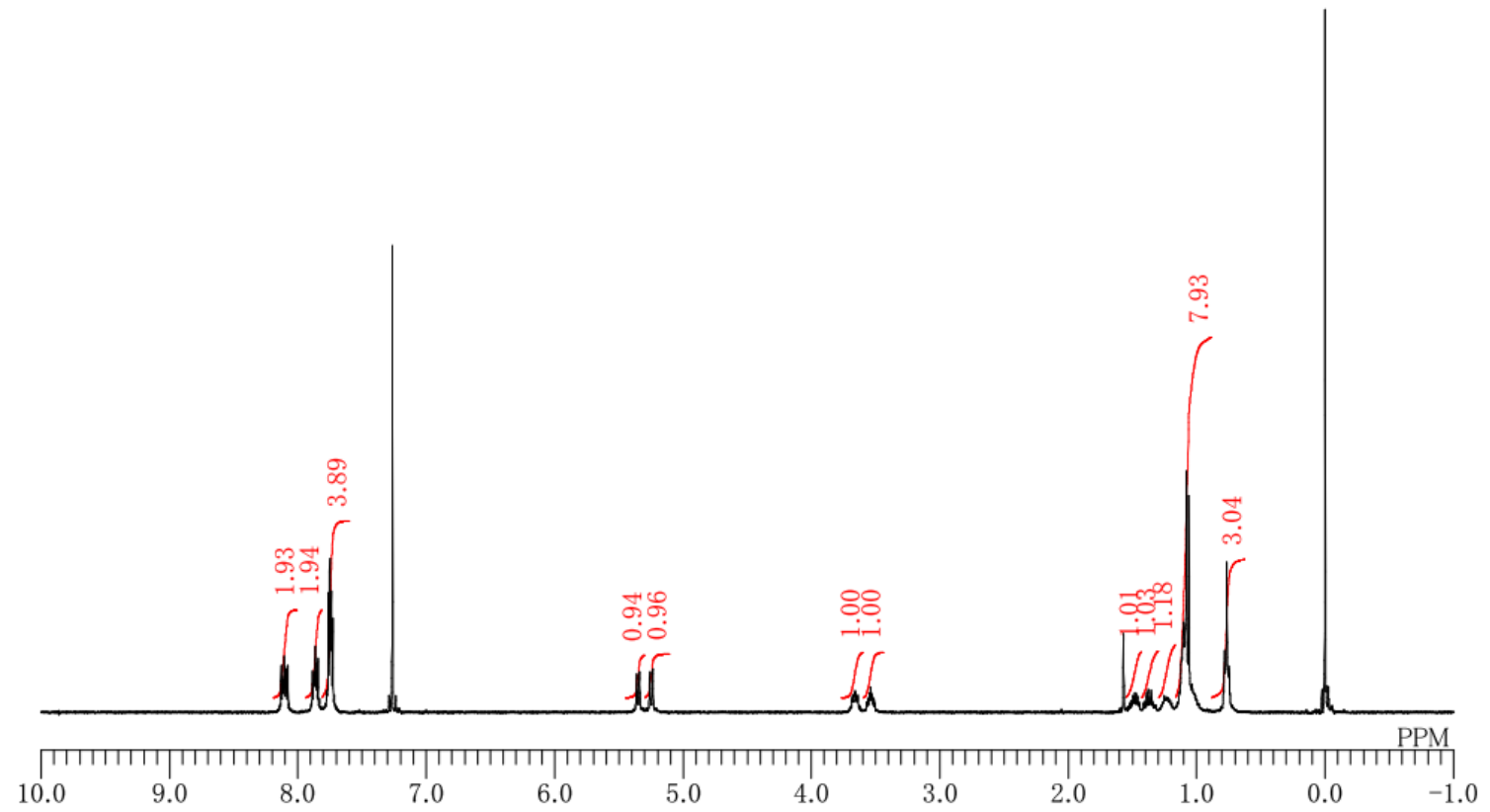

${ }^{13} \mathrm{C}$ NMR $\left(100 \mathrm{MHz}, \mathrm{CDCl}_{3}\right)$

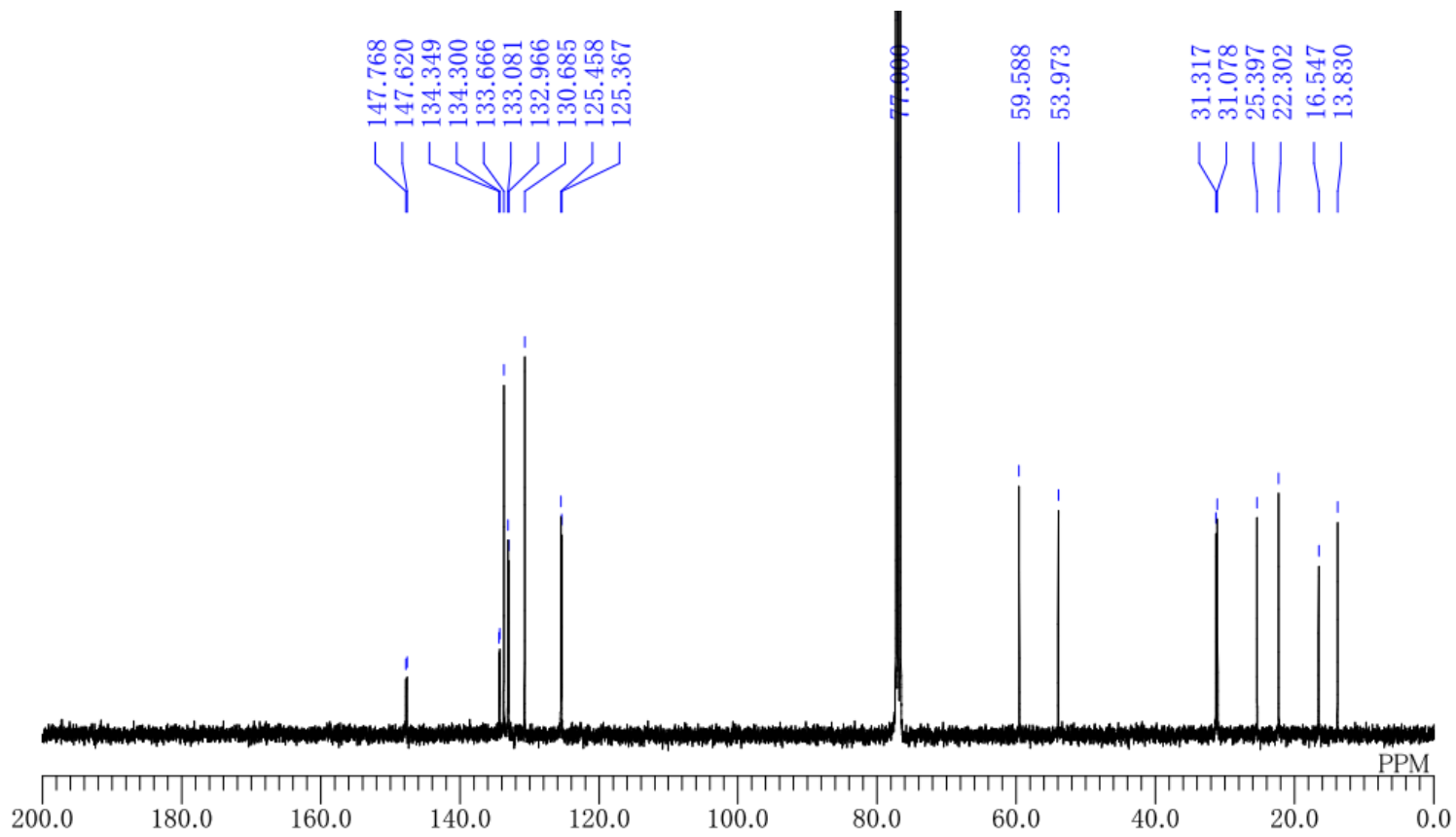


$N, N^{\prime}-\left(\left(2 R^{*}, 3 R^{*}\right)\right.$-octane-2,3-diyl)bis(2-nitrobenzenesulfonamide) (4i)<smiles>CC(N)C(C)NS(=O)(=O)c1ccccc1</smiles>

${ }^{1} \mathrm{H}$ NMR (400 MHz, $\left.\mathrm{CDCl}_{3}\right)$

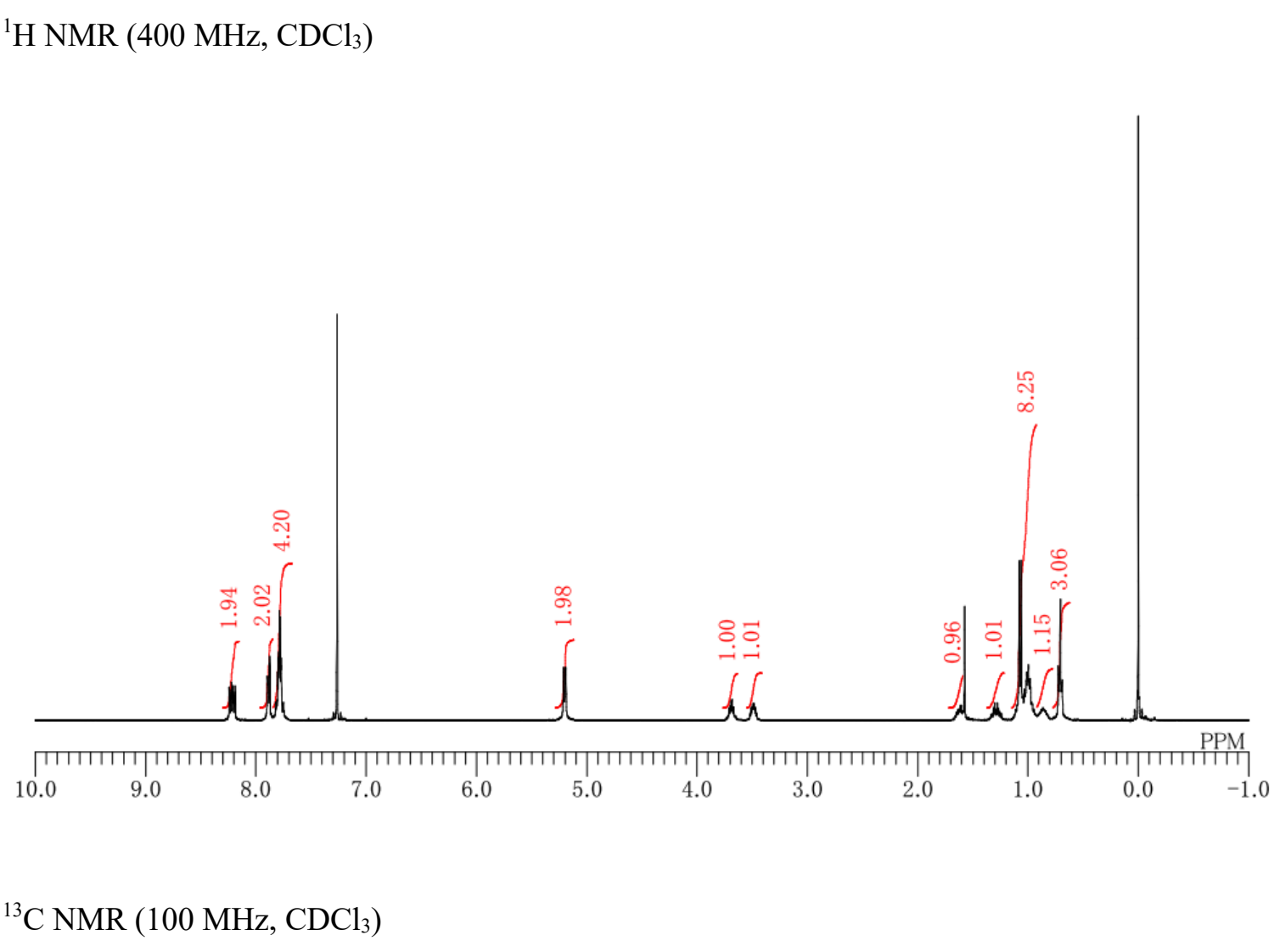

${ }^{13} \mathrm{C}$ NMR $\left(100 \mathrm{MHz}, \mathrm{CDCl}_{3}\right)$
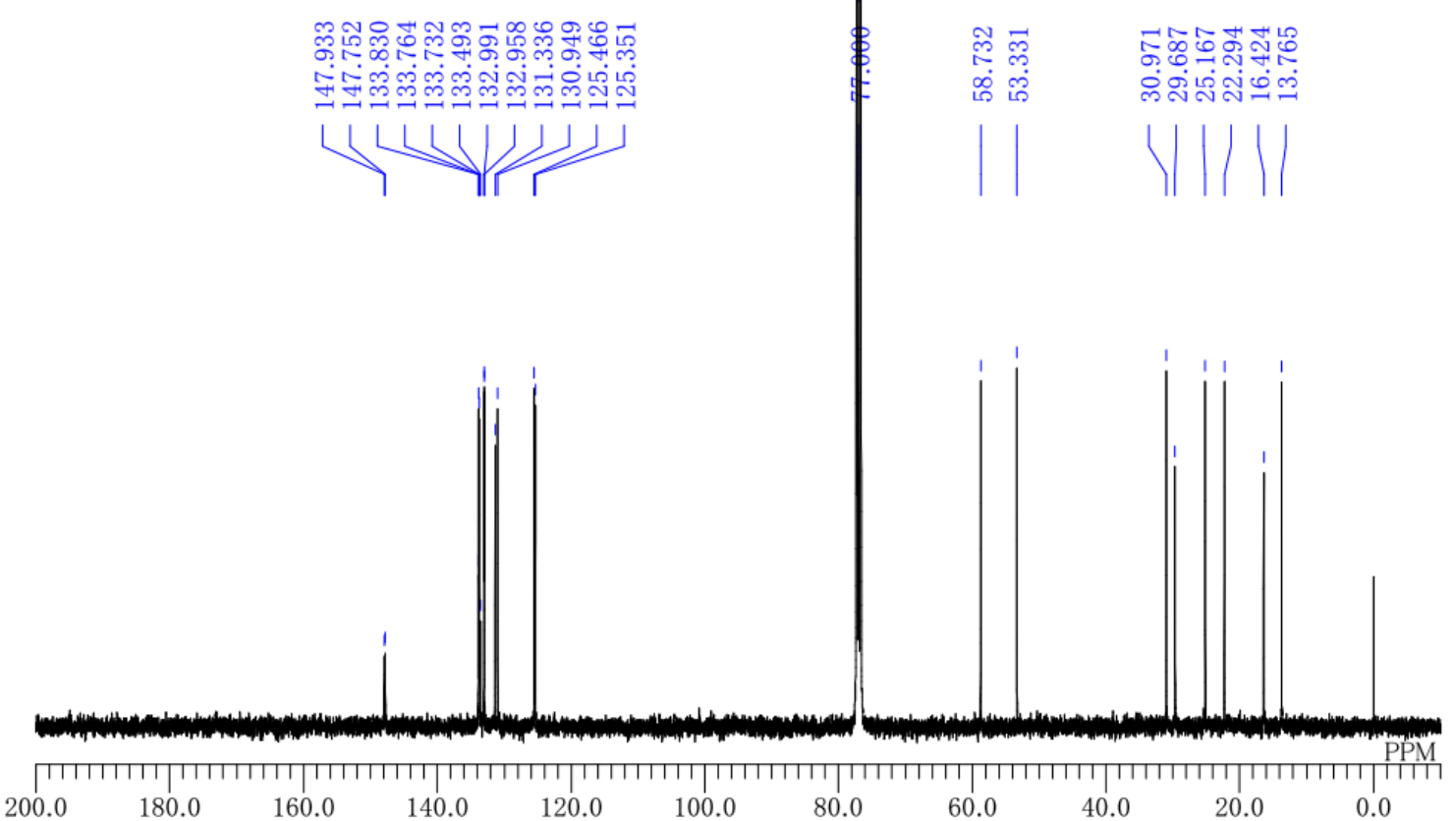
$N, N^{\prime}-\left(\left(1 R^{*}, 2 S^{*}\right)\right.$-1-phenylpropane-1,2-diyl)bis(2-nitrobenzenesulfonamide) (4j)<smiles>CC(C(c1ccccc1)[N+](=O)[O-])[N+](=O)[O-]</smiles>

${ }^{1} \mathrm{H}$ NMR (400 MHz, DMSO- $\left.d_{6}\right)$

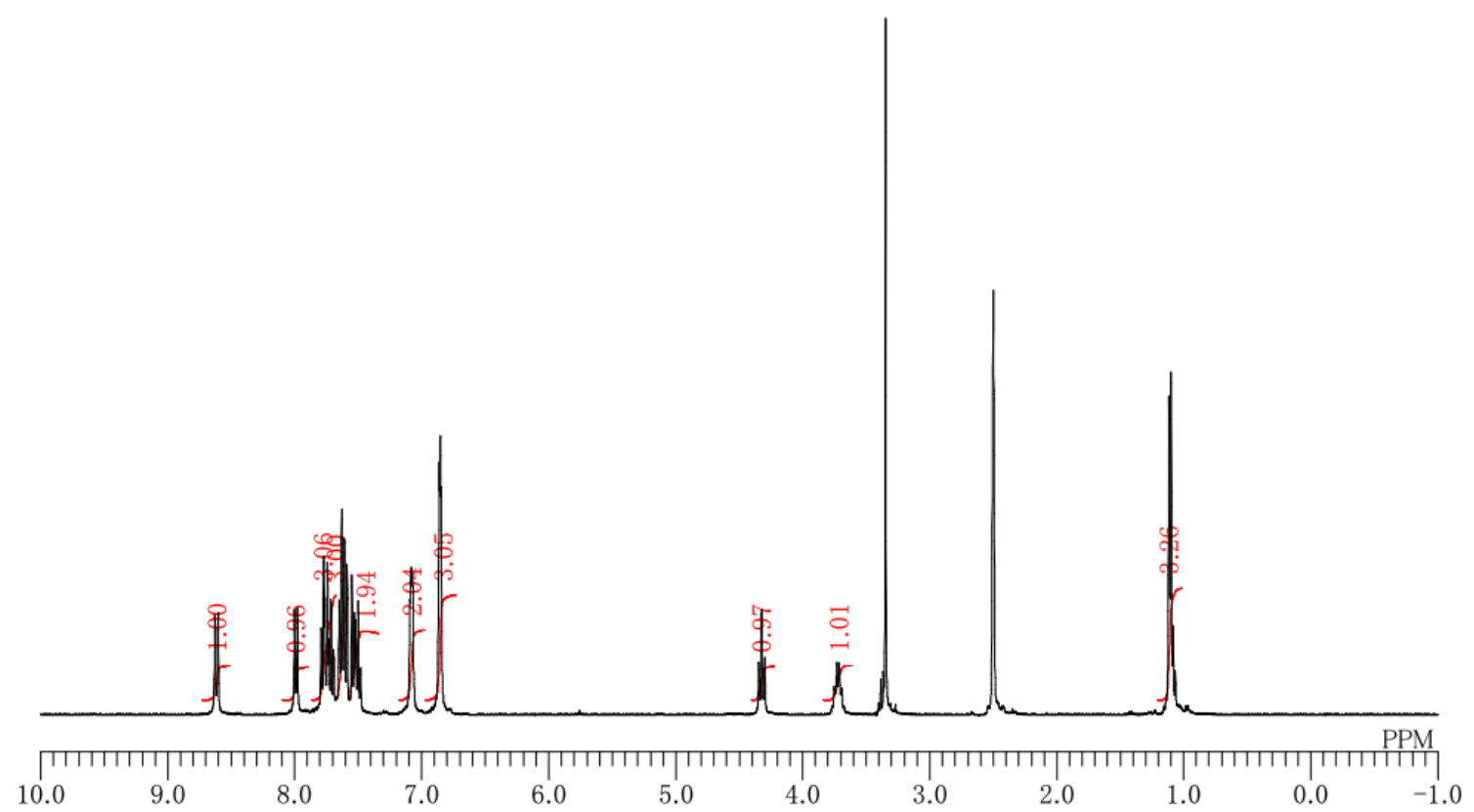

${ }^{13} \mathrm{C}$ NMR $\left(100 \mathrm{MHz}\right.$, DMSO- $\left.d_{6}\right)$
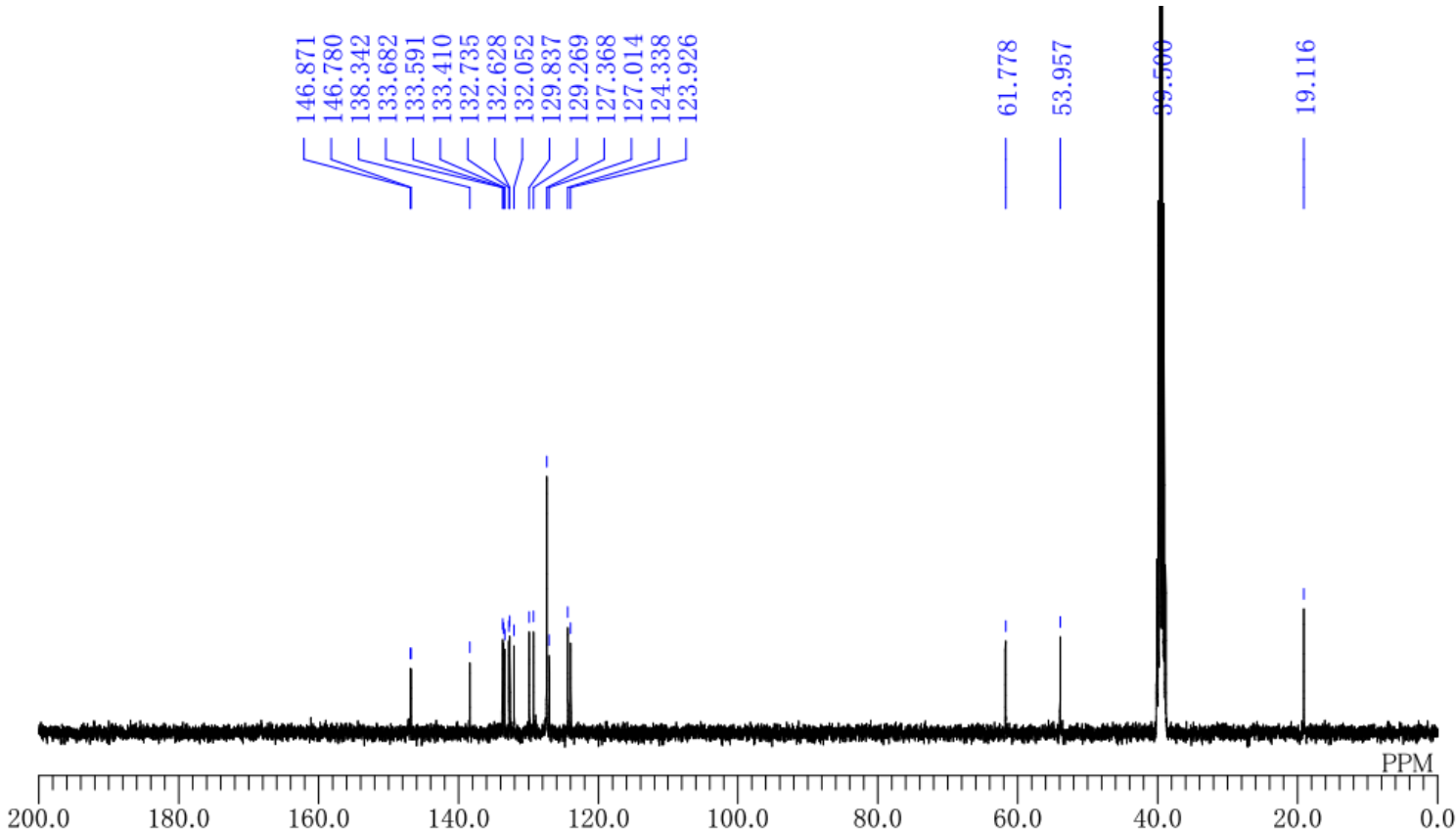
$N, N^{\prime}$-((1 $\left.\left.R^{*}, 2 R^{*}\right)-1-p h e n y l p r o p a n e-1,2-d i y l\right) b i s(2-n i t r o b e n z e n e s u l f o n a m i d e) ~(4 k)$<smiles>CC(NS(C)(=O)=O)C(c1ccccc1)c1ccccc1</smiles>

${ }^{1} \mathrm{H}$ NMR (400 MHz, DMSO- $\left.d_{6}\right)$

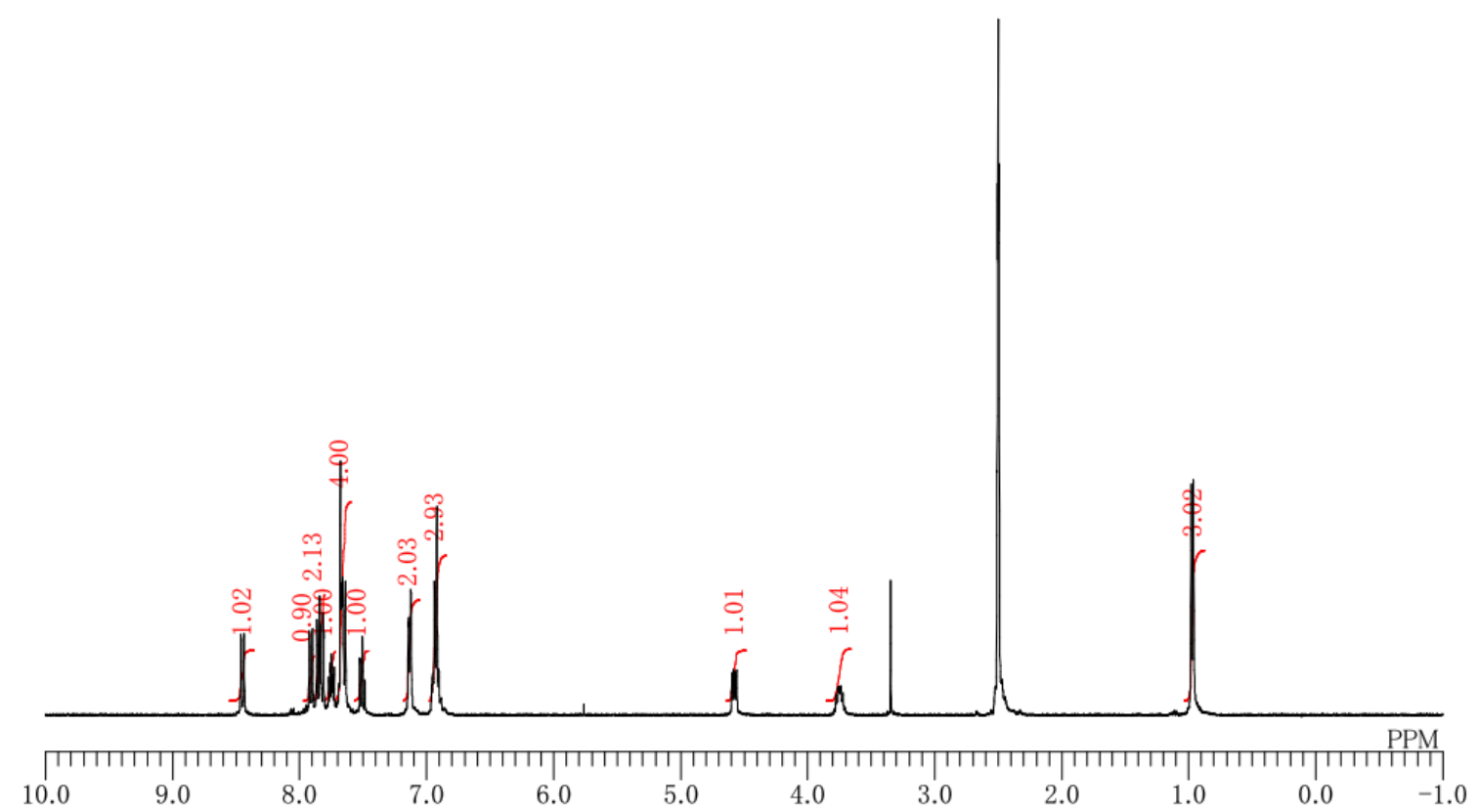

${ }^{13} \mathrm{C}$ NMR $\left(100 \mathrm{MHz}\right.$, DMSO- $\left.d_{6}\right)$
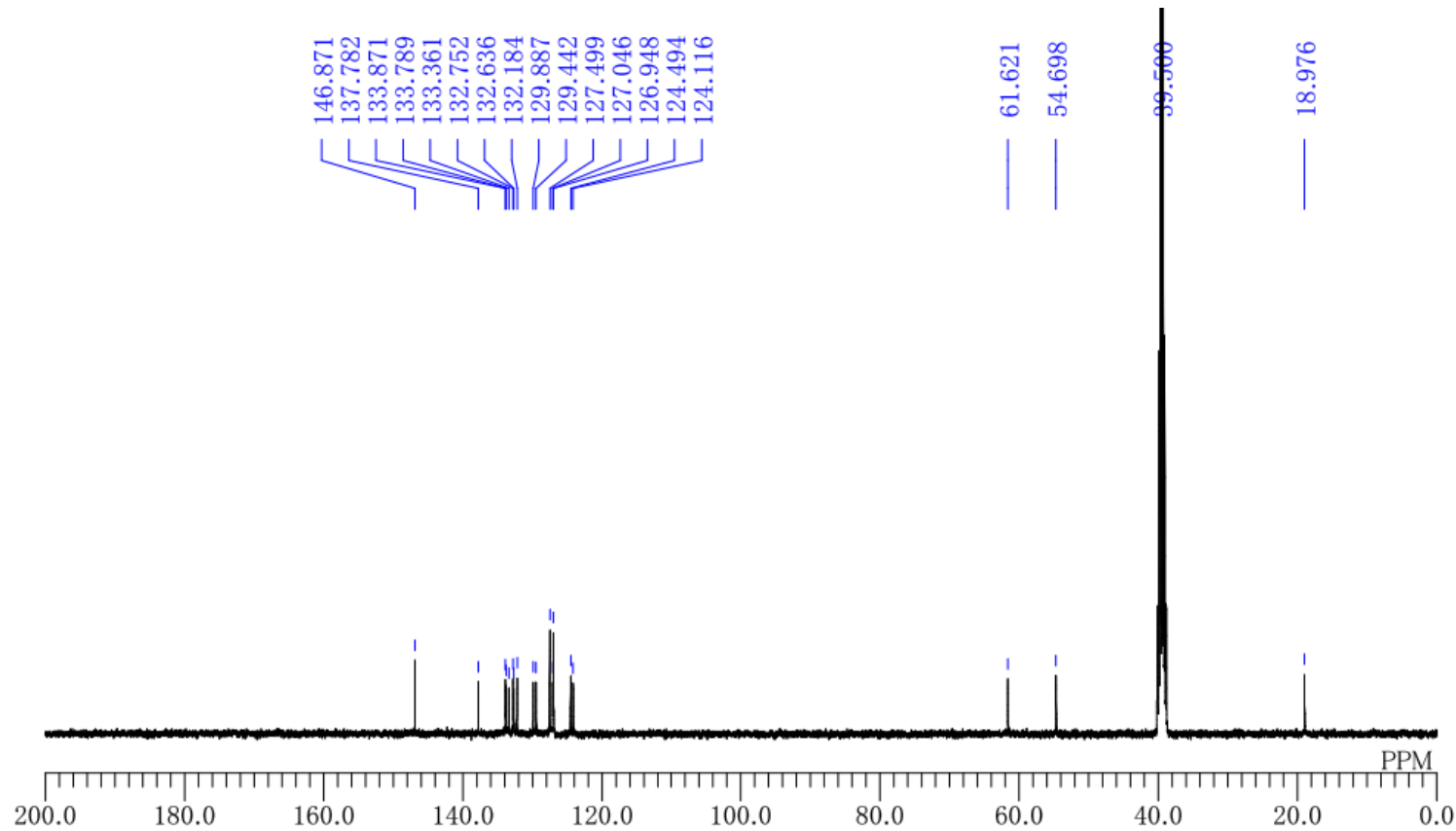


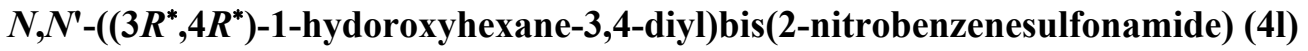<smiles>CCC(NS(C)(=O)=O)C(CCO)[N+](=O)[O-]</smiles>

${ }^{1} \mathrm{H}$ NMR (400 MHz, DMSO- $\left.d_{6}\right)$

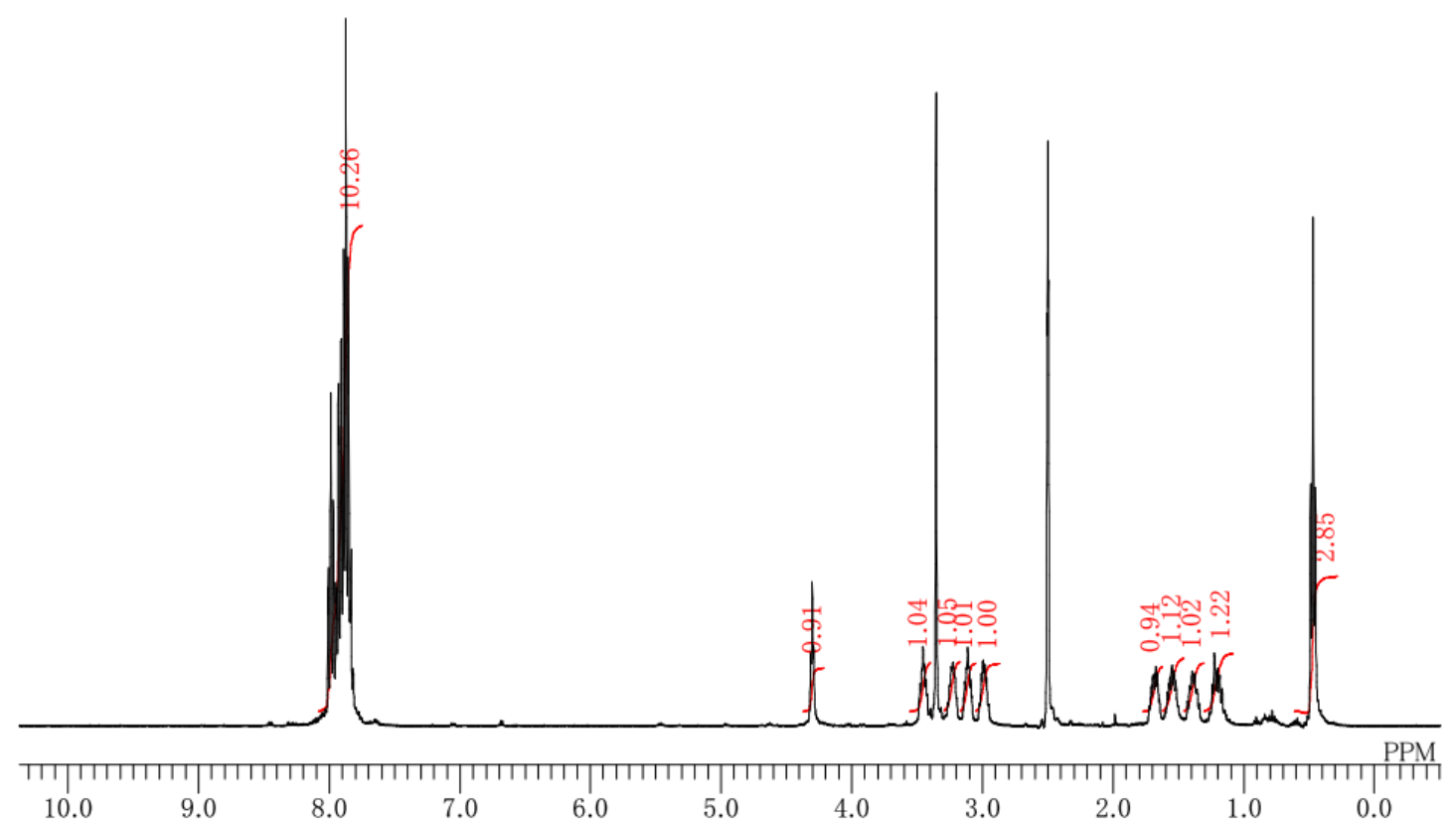

${ }^{13} \mathrm{C}$ NMR $\left(100 \mathrm{MHz}\right.$, DMSO- $\left.d_{6}\right)$
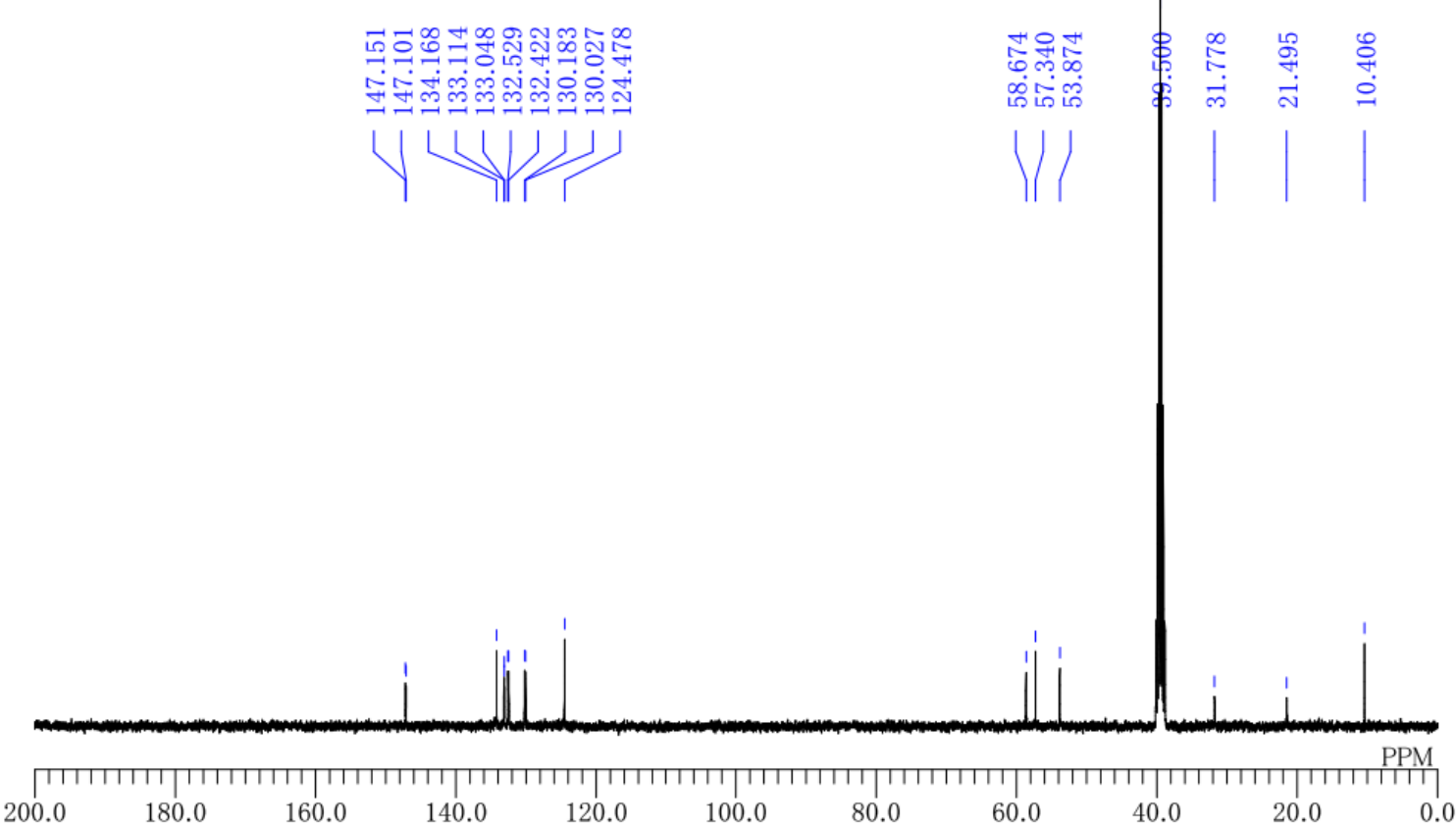
$N, N^{\prime}-\left(\left(2 R^{*}, 3 R^{*}\right)-1,4-d i h y d o r o x y b u t a n e-2,3-d i y l\right) b i s(2-n i t r o b e n z e n e s u l f o n a m i d e)(4 \mathrm{~m})$<smiles>[N-]S(=O)(=O)C(CO)C(CO)[N+](=O)[O-]</smiles>

${ }^{1} \mathrm{H}$ NMR (400 MHz, DMSO- $\left.d_{6}\right)$

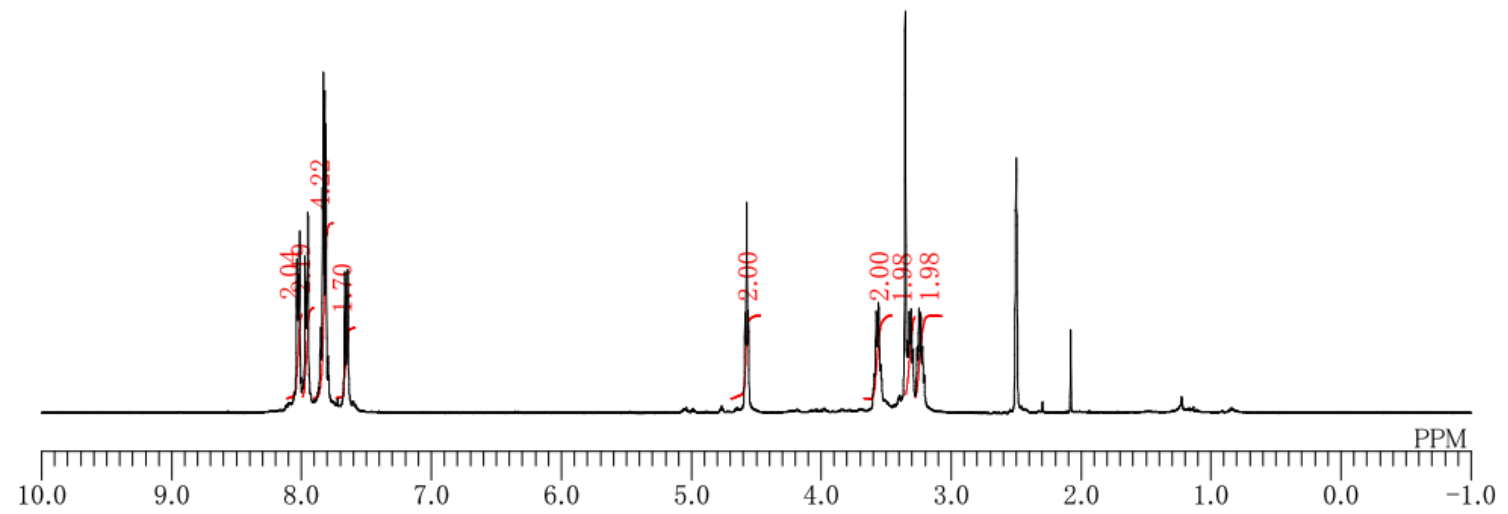

${ }^{13} \mathrm{C}$ NMR $\left(100 \mathrm{MHz}\right.$, DMSO- $\left.d_{6}\right)$
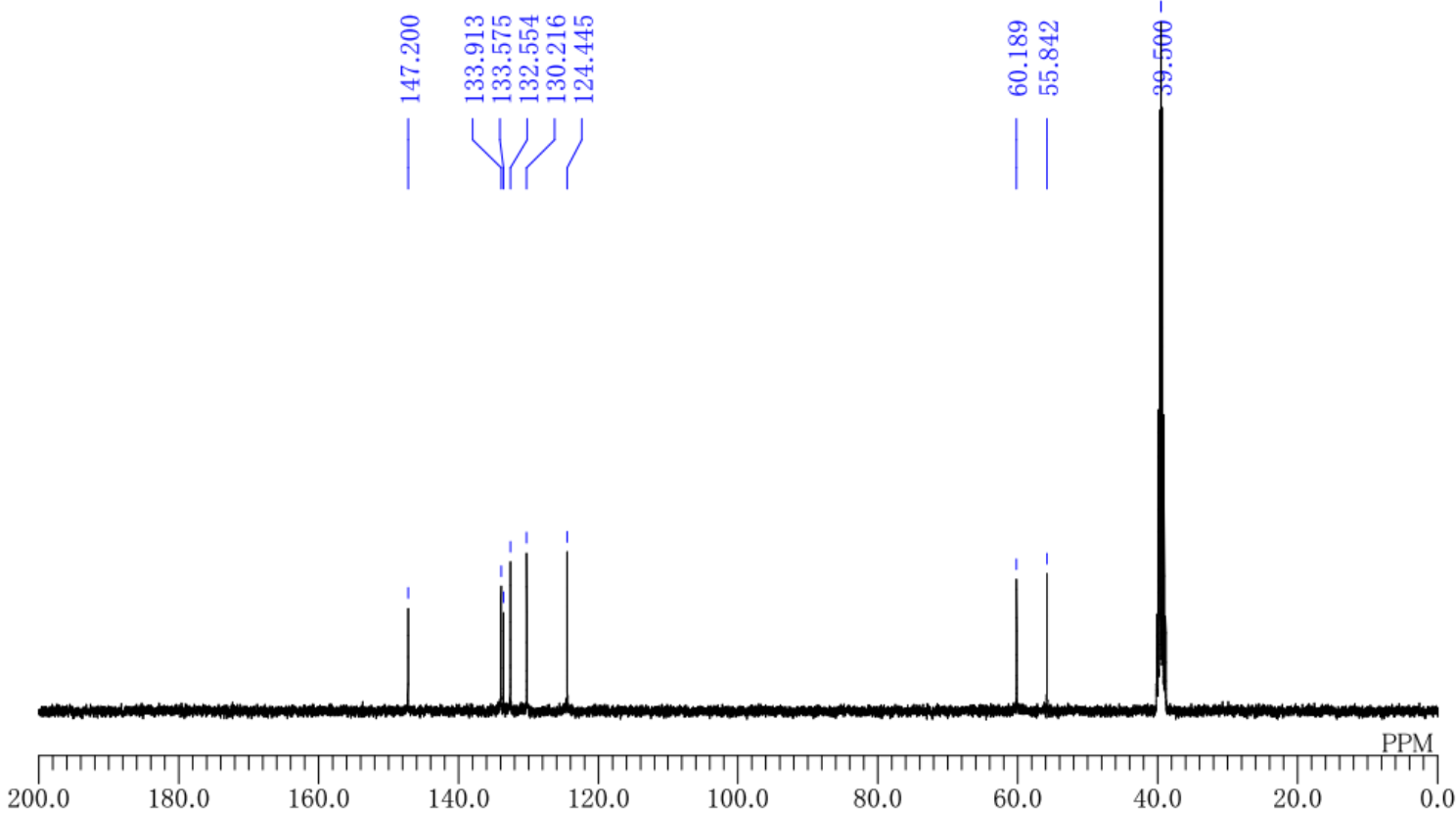


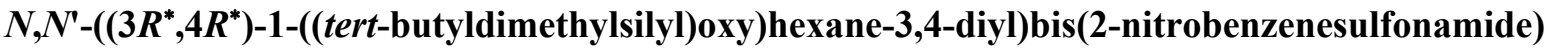

(4n)<smiles>CCC(NS(=O)(=O)[O-])C(CCOC(C)(C)C)NS(C)(=O)=O</smiles>

${ }^{1} \mathrm{H} \mathrm{NMR}\left(400 \mathrm{MHz}, \mathrm{CDCl}_{3}\right)$

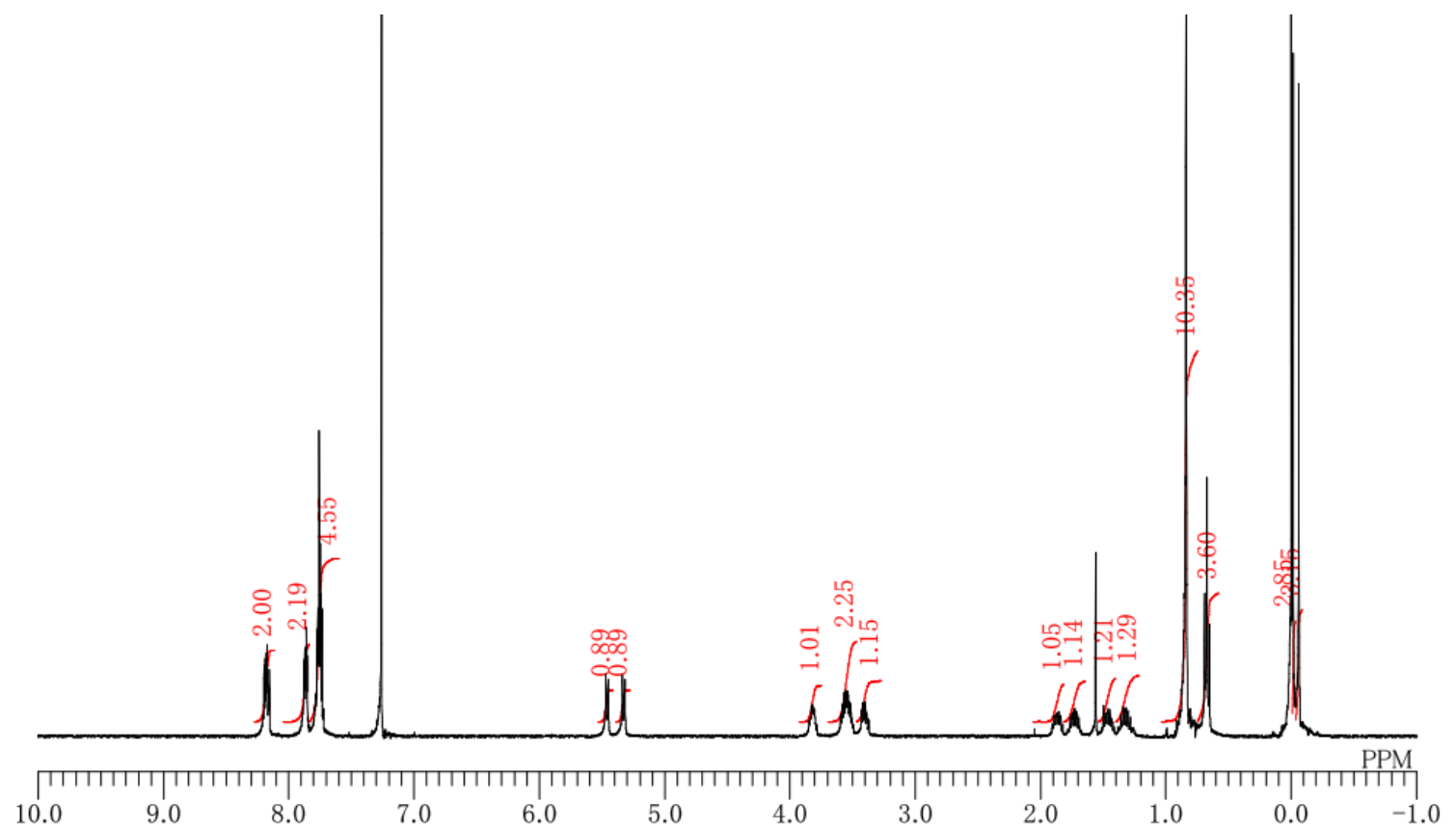

${ }^{13} \mathrm{C}$ NMR $\left(100 \mathrm{MHz}, \mathrm{CDCl}_{3}\right)$

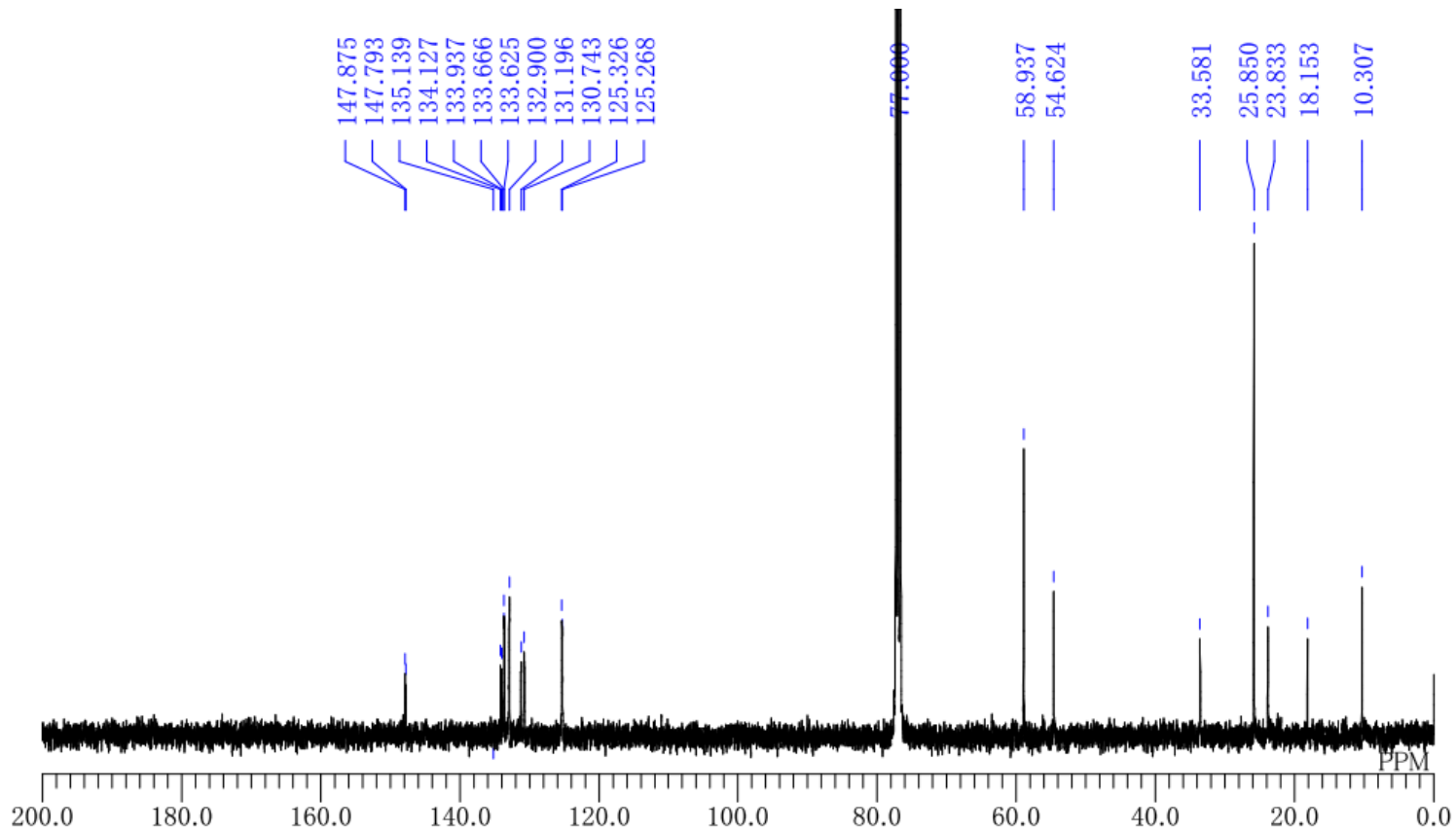


$N, N^{\prime}$-(2-methylhexane-2,3-diyl)bis(2-nitrobenzenesulfonamide) (40)<smiles>CCCCC(C)(C)C(C)(NS(=O)(=O)[O-])S(=O)(=O)c1ccccc1</smiles>

${ }^{1} \mathrm{H}$ NMR $\left(400 \mathrm{MHz}, \mathrm{CDCl}_{3}\right)$

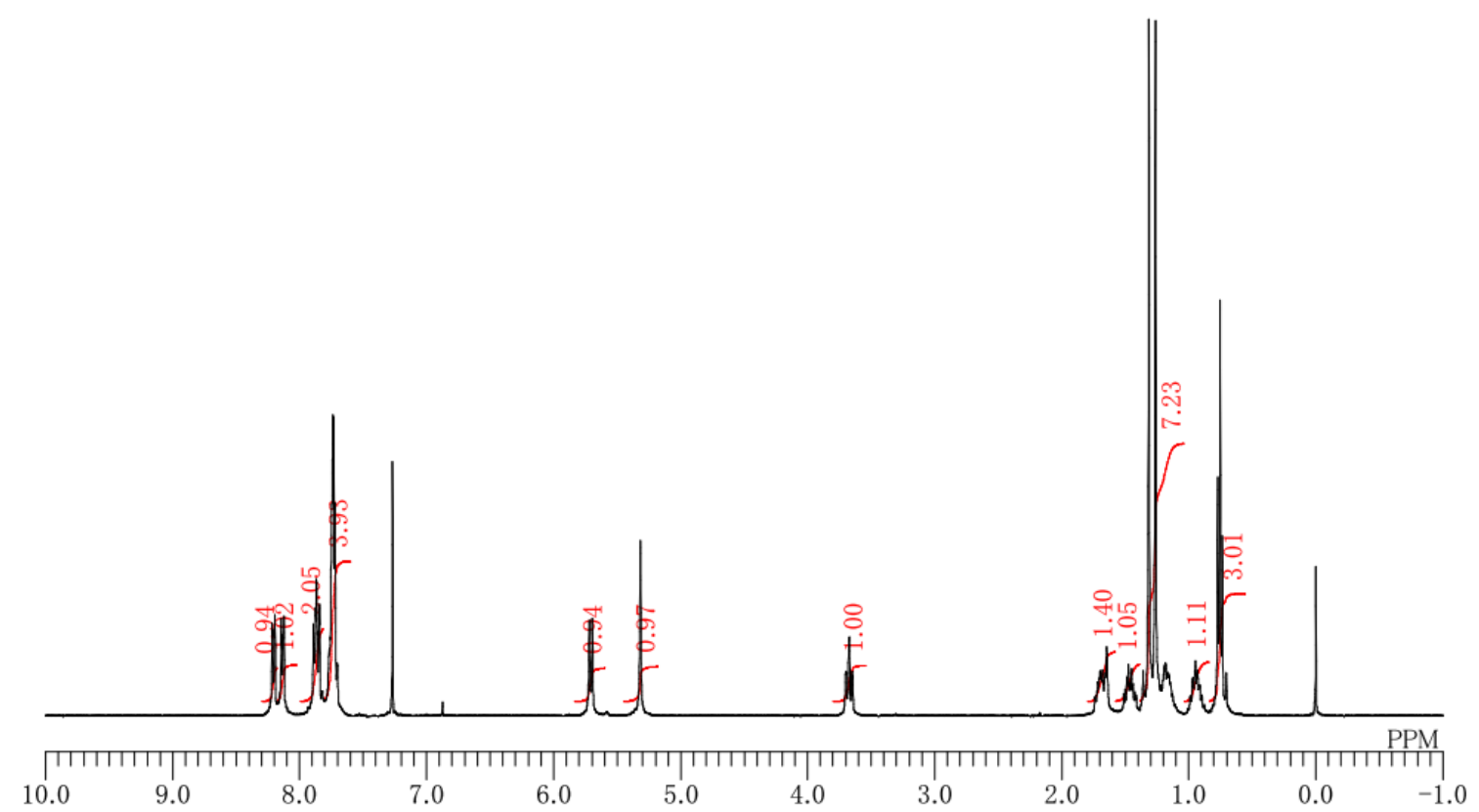

${ }^{13} \mathrm{C}$ NMR $\left(100 \mathrm{MHz}, \mathrm{CDCl}_{3}\right)$

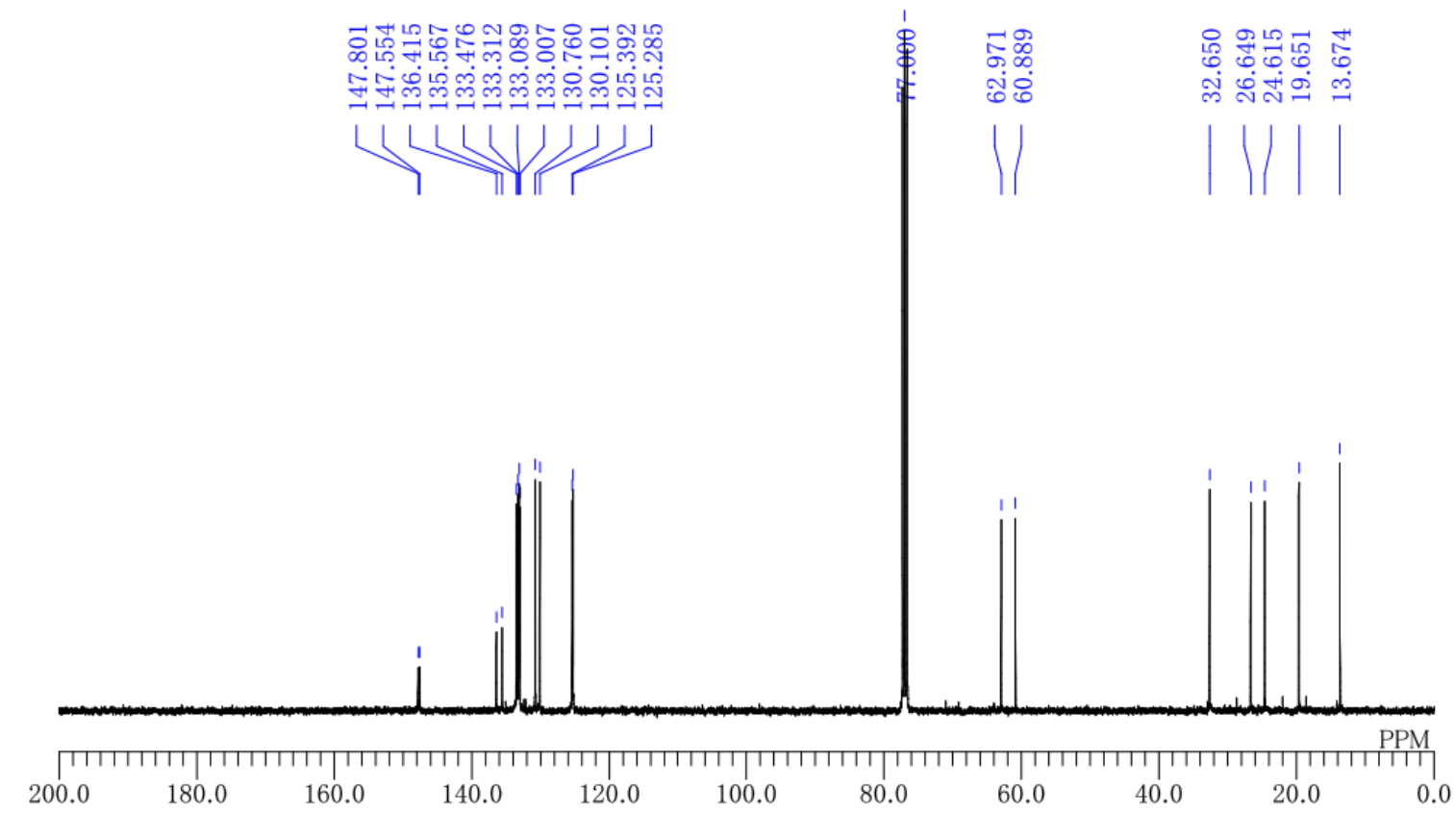


$N, N^{\prime}$-((1 $\left.R^{*}, 2 R^{*}\right)-1,2$-diphenylethane-1,2-diyl)bis(2-nitrobenzenesulfonamide) (4p)<smiles>O=[N+](c1ccccc1)C(c1ccccc1)c1ccccc1</smiles>

${ }^{1} \mathrm{H}$ NMR (400 MHz, DMSO- $\left.d_{6}\right)$

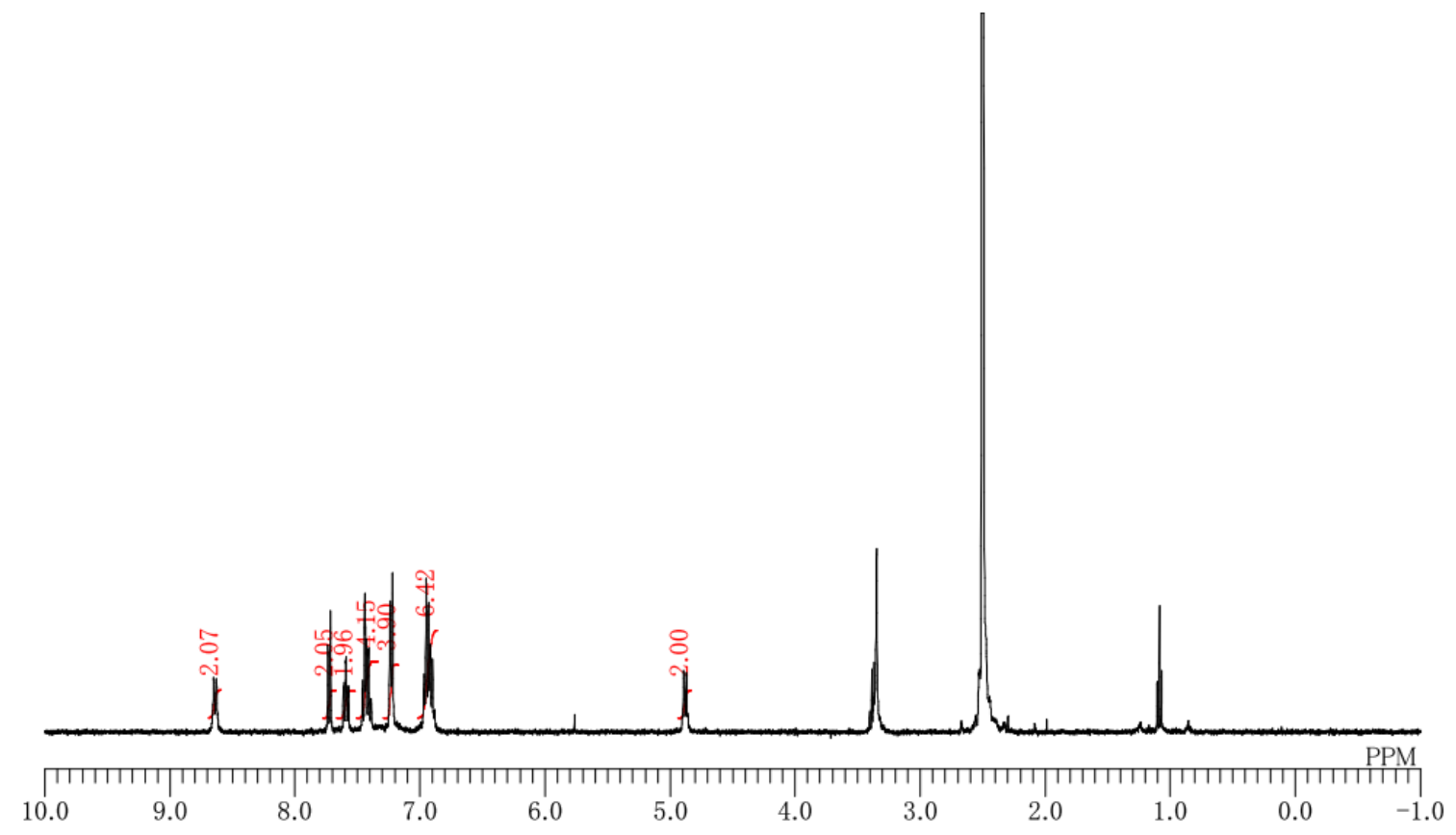

${ }^{13} \mathrm{C}$ NMR $\left(100 \mathrm{MHz}\right.$, DMSO- $\left.d_{6}\right)$
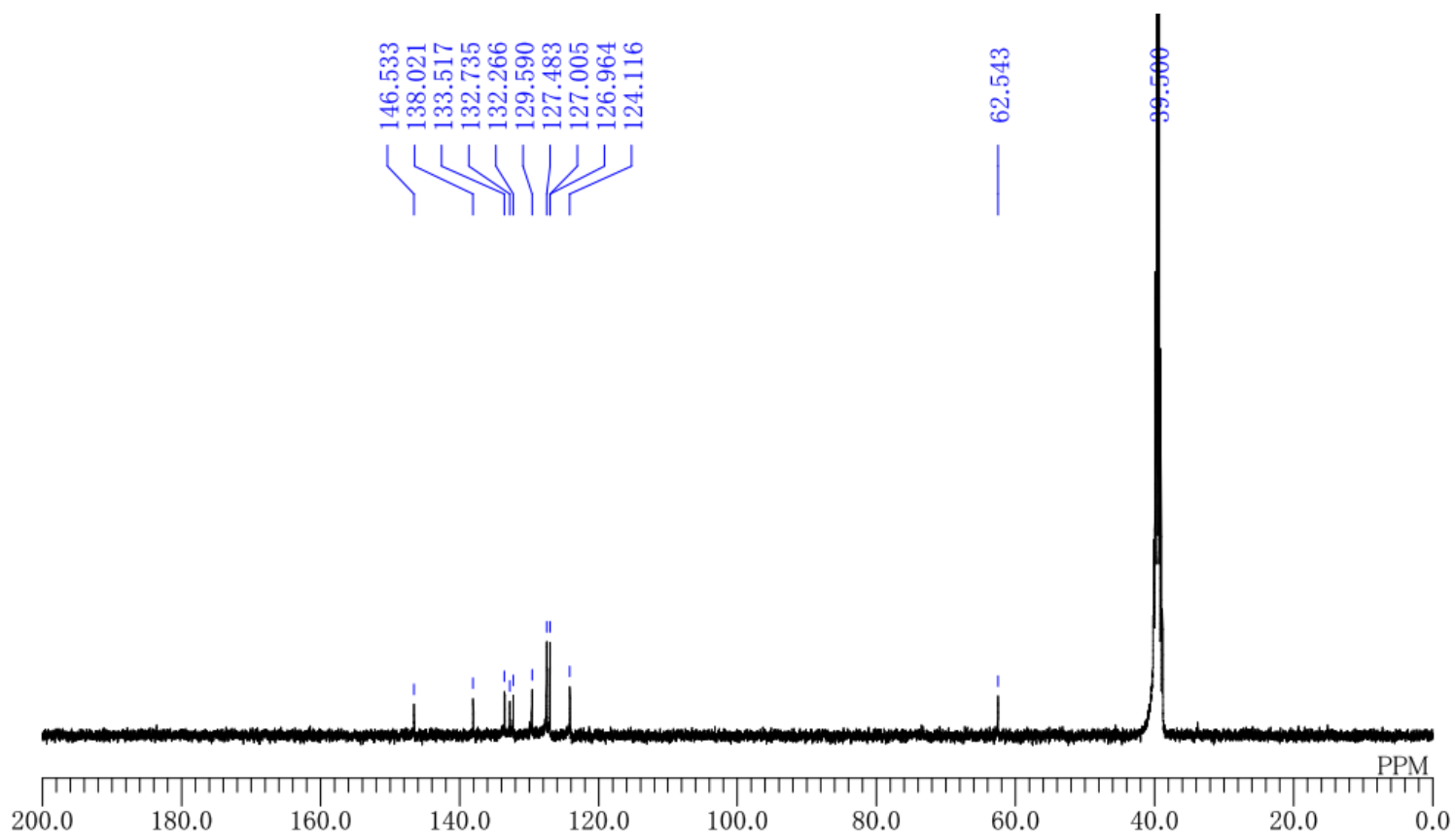
di-tert-butyl $\left(3 \mathrm{a} R^{*}, 6 \mathrm{a} S^{*}\right)$-cyclopenta[c] $[1,2,5]$ thiadiazolidine-1,3-dicarboxylate 2,2-dioxide (5a)

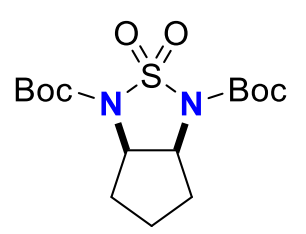

${ }^{1} \mathrm{H}$ NMR $\left(400 \mathrm{MHz}, \mathrm{CDCl}_{3}\right)$

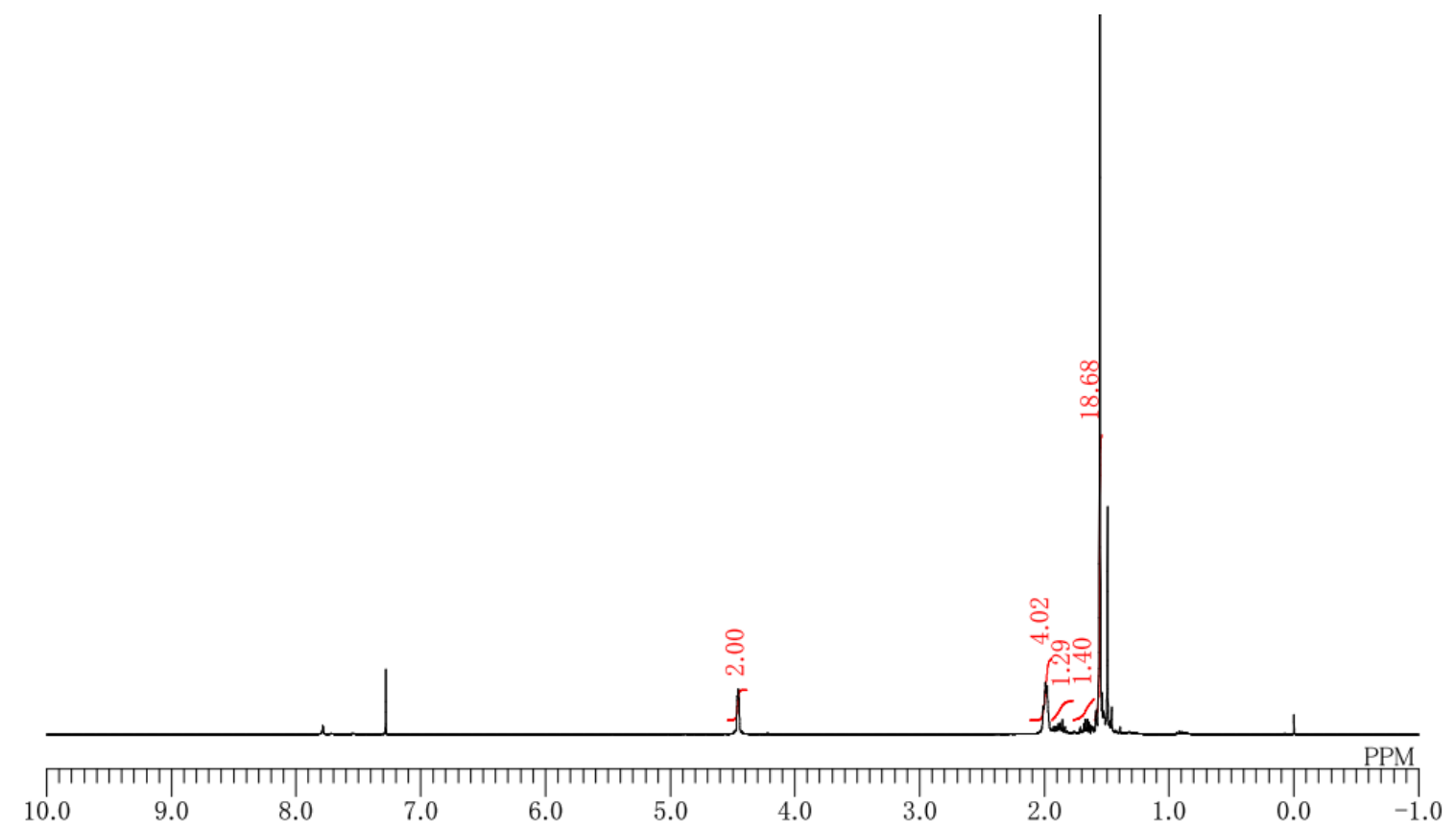

${ }^{13} \mathrm{C}$ NMR $\left(100 \mathrm{MHz}, \mathrm{CDCl}_{3}\right)$

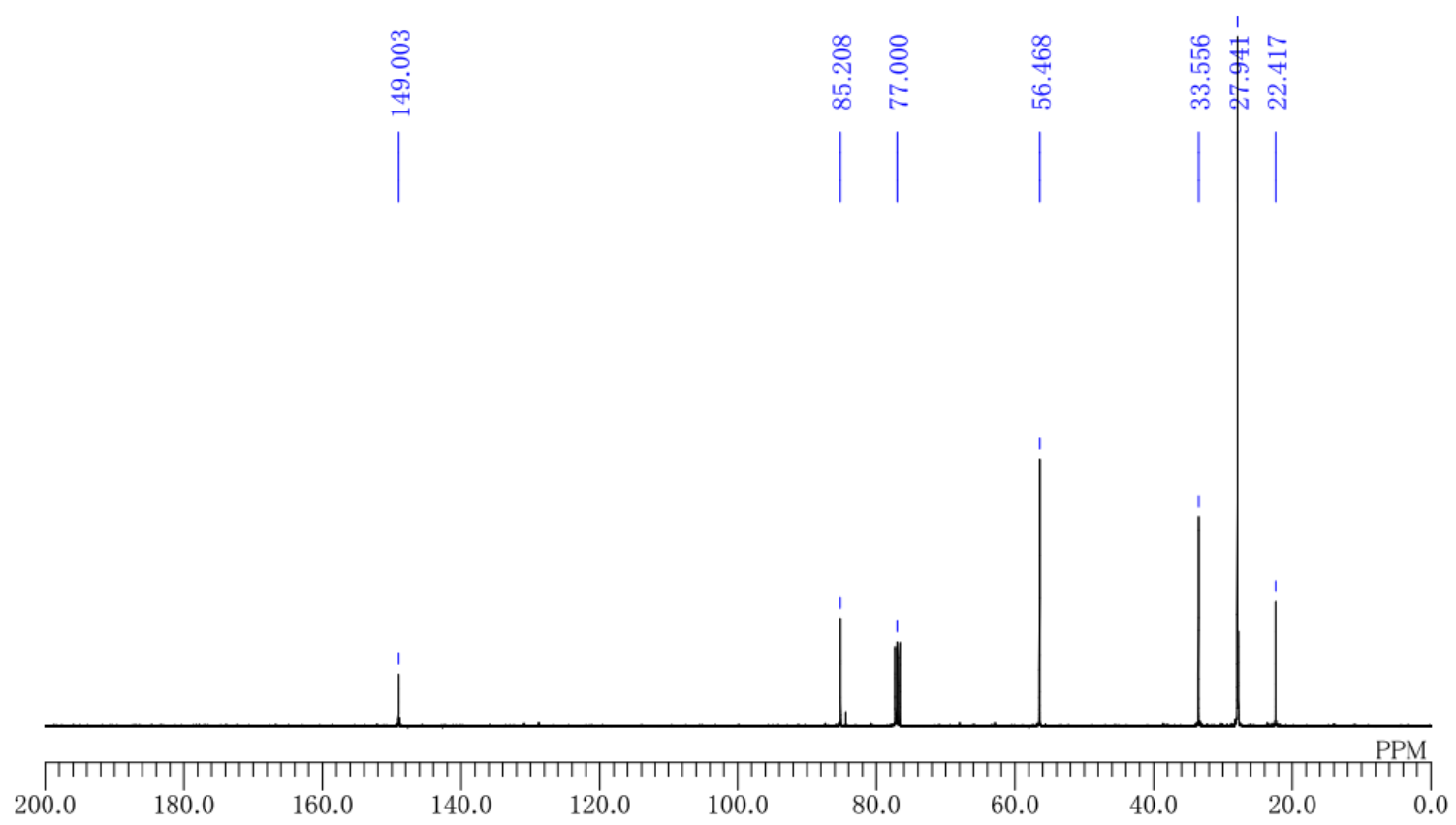


di-tert-butyl $\left(3 \mathrm{a} R^{*}, 7 \mathrm{a} S^{*}\right)$-cyclohexa[c][1,2,5]thiadiazolidine-1,3-dicarboxylate 2,2-dioxide (5b)

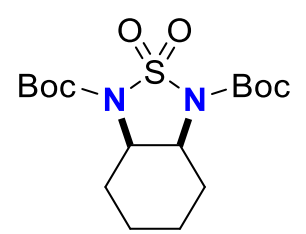

${ }^{1} \mathrm{H}$ NMR $\left(400 \mathrm{MHz}, \mathrm{CDCl}_{3}\right)$

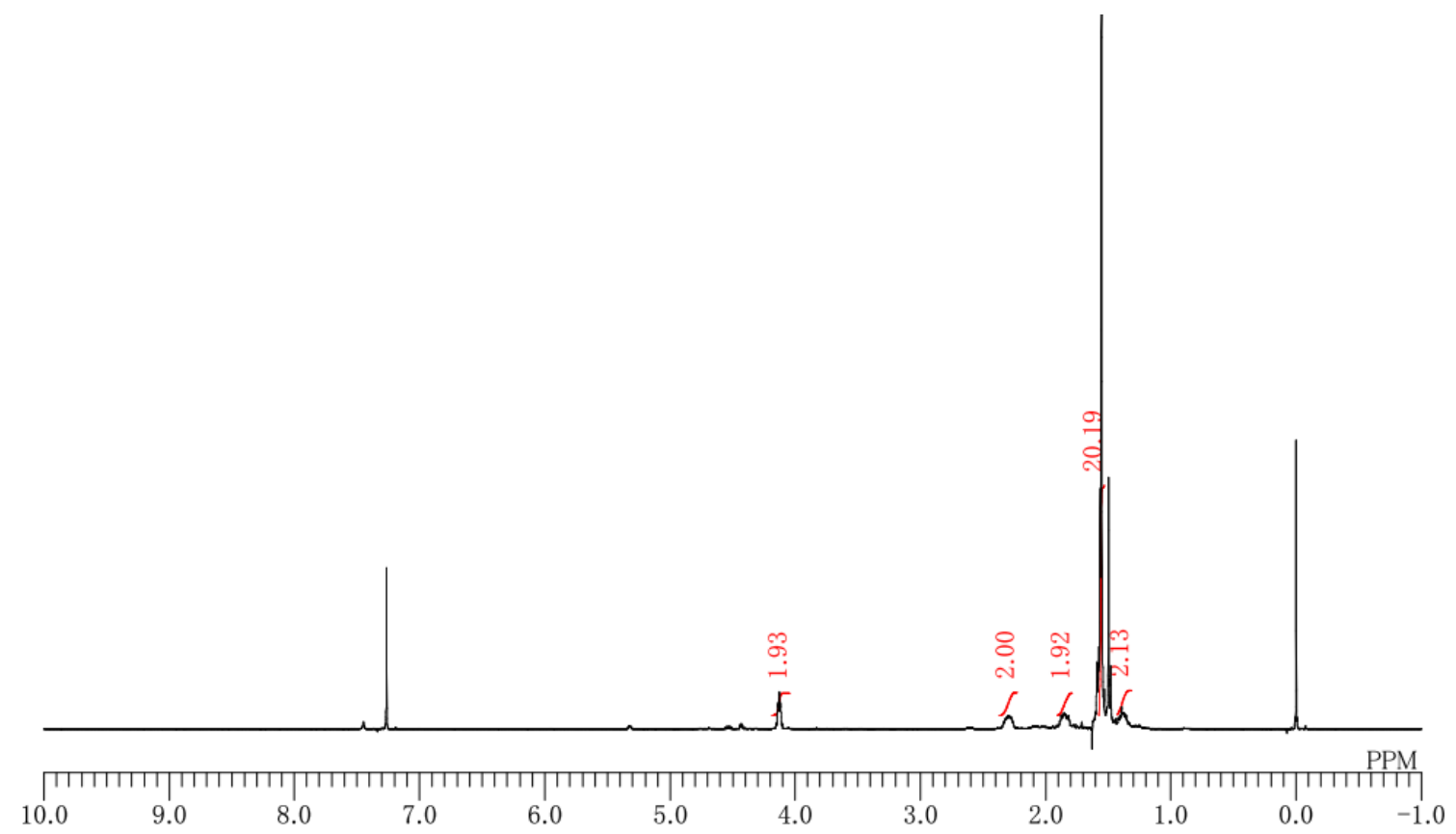

${ }^{13} \mathrm{C}$ NMR $\left(100 \mathrm{MHz}, \mathrm{CDCl}_{3}\right)$

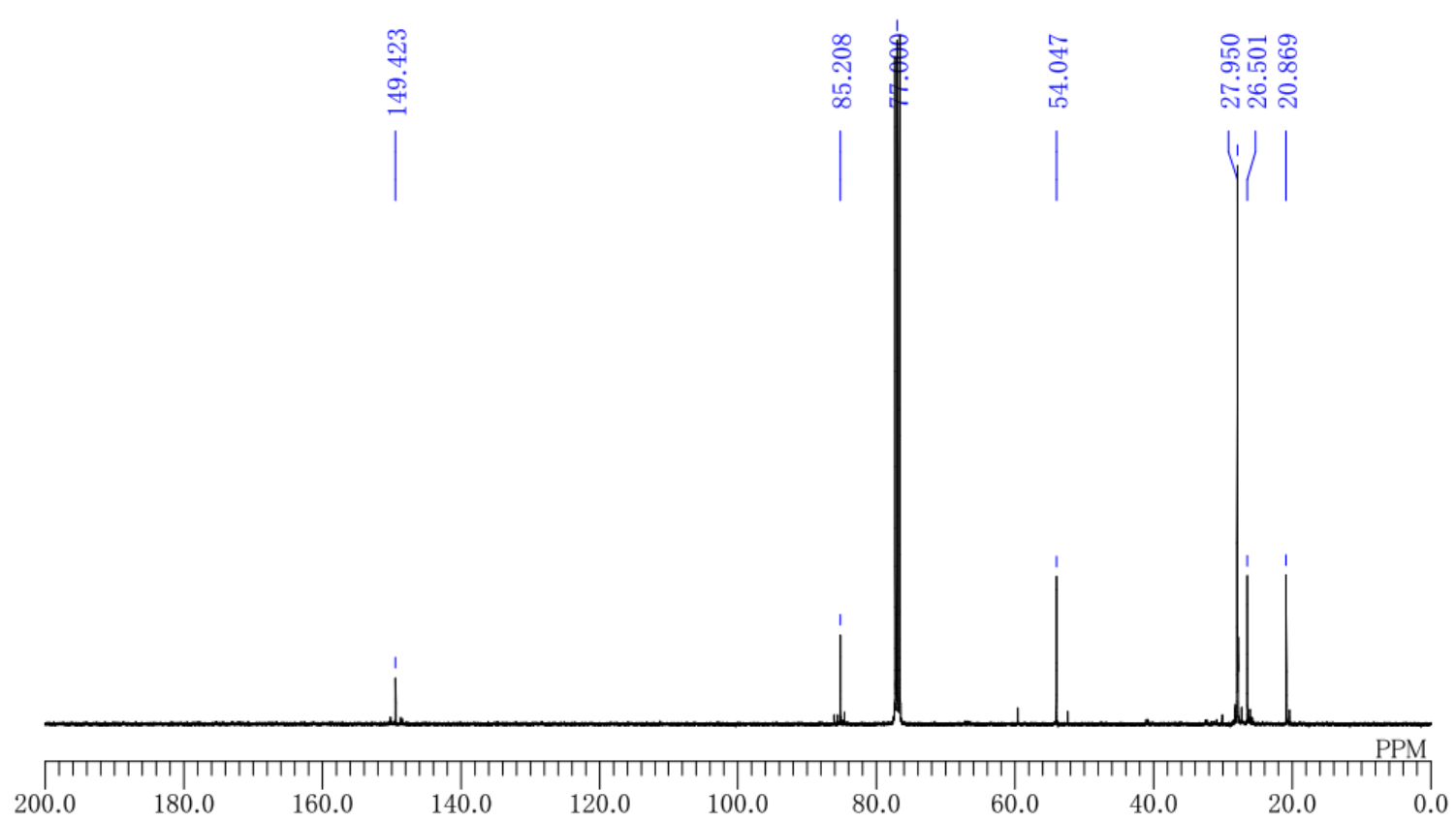


di-tert-butyl $\left(3 \mathrm{a} R^{*}, 8 \mathrm{a} S^{*}\right)$-cyclohepta $[c][1,2,5]$ thiadiazolidine-1,3-dicarboxylate 2,2-dioxide (5q)

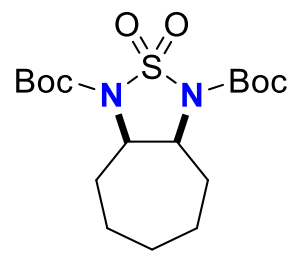

${ }^{1} \mathrm{H}$ NMR $\left(400 \mathrm{MHz}, \mathrm{CDCl}_{3}\right)$

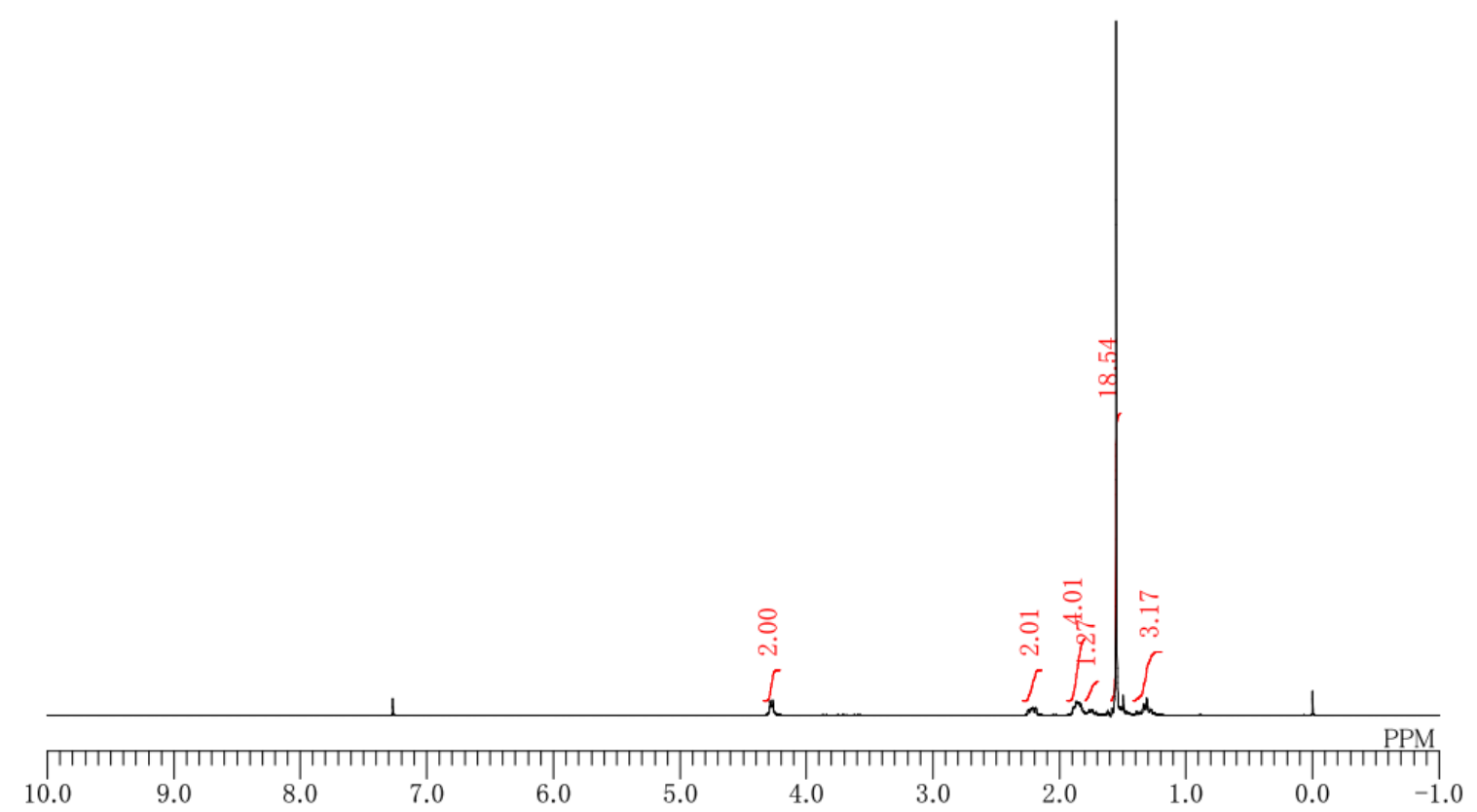

${ }^{13} \mathrm{C}$ NMR $\left(100 \mathrm{MHz}, \mathrm{CDCl}_{3}\right)$

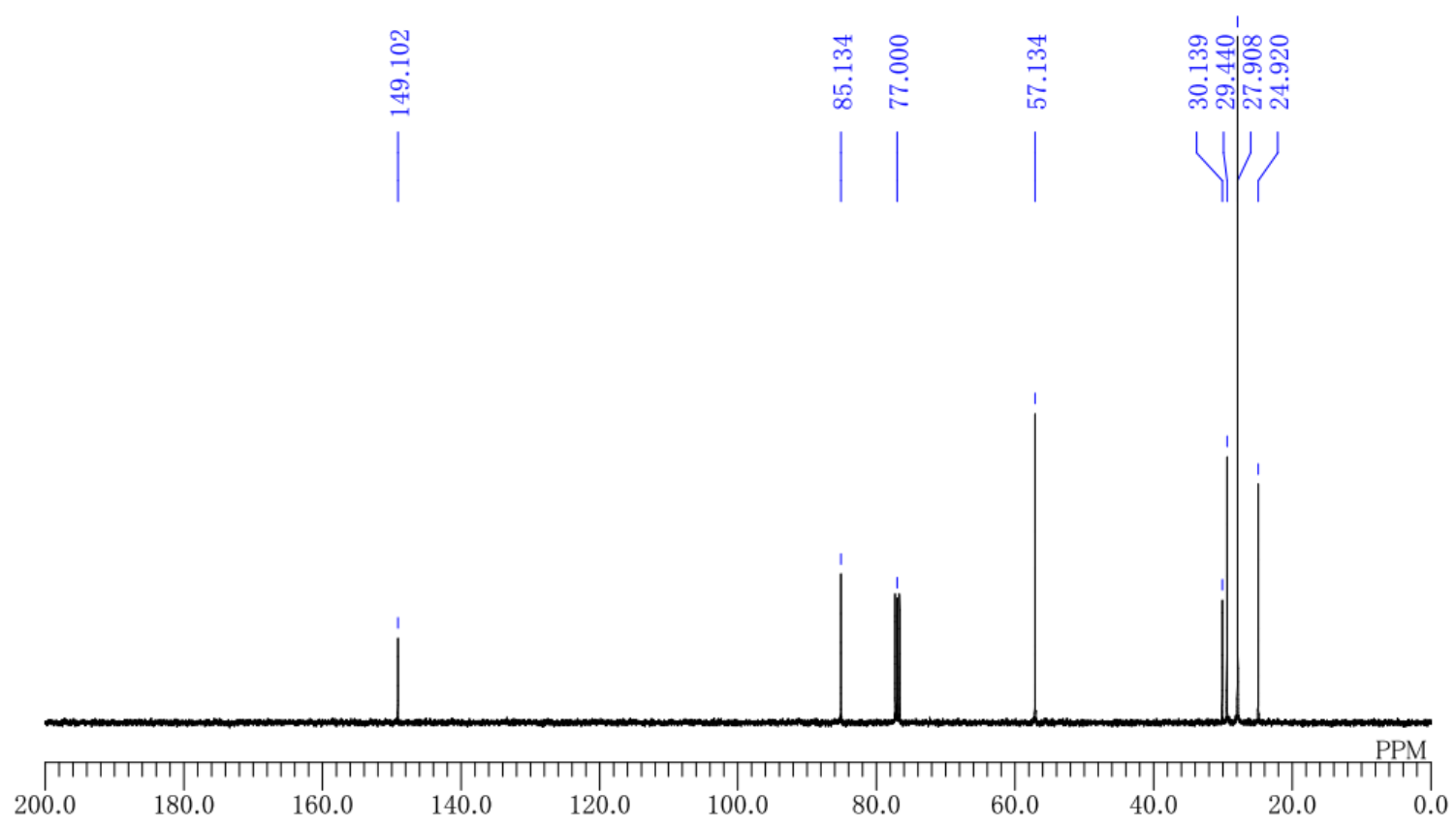


di-tert-butyl $\left(3 \mathrm{a} R^{*}, 8 \mathrm{~b} S^{*}\right)$-inda[1,2-c][1,2,5]thiadiazolidine-1,3-dicarboxylate 2,2-dioxide (5e)<smiles>CC(C)(C)OC(=O)N1C2Cc3ccccc3C2N(C(=O)OC(C)(C)C)S1(=O)=O</smiles>

${ }^{1} \mathrm{H}$ NMR $\left(400 \mathrm{MHz}, \mathrm{CDCl}_{3}\right)$

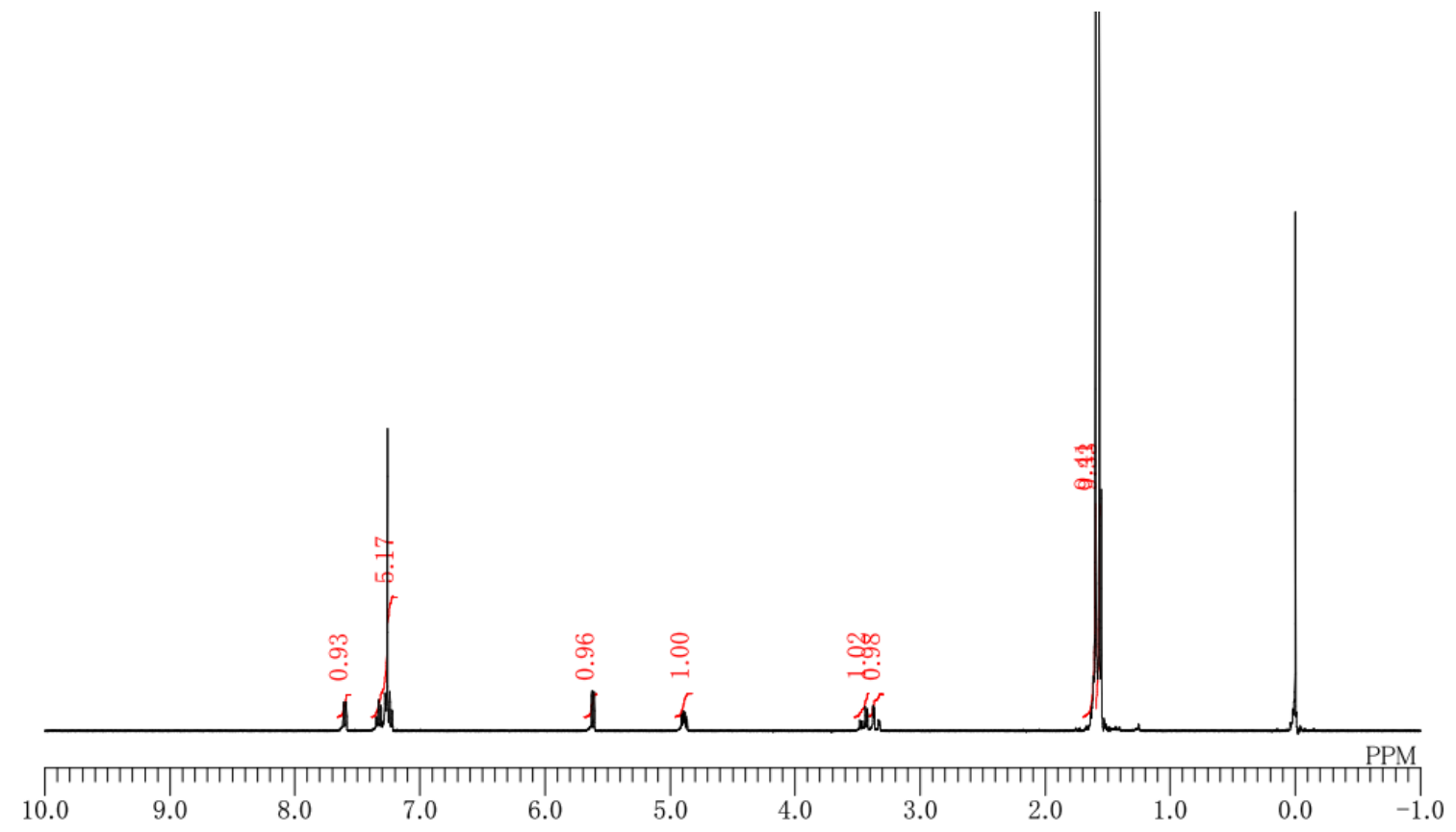

${ }^{13} \mathrm{C}$ NMR $\left(100 \mathrm{MHz}, \mathrm{CDCl}_{3}\right)$

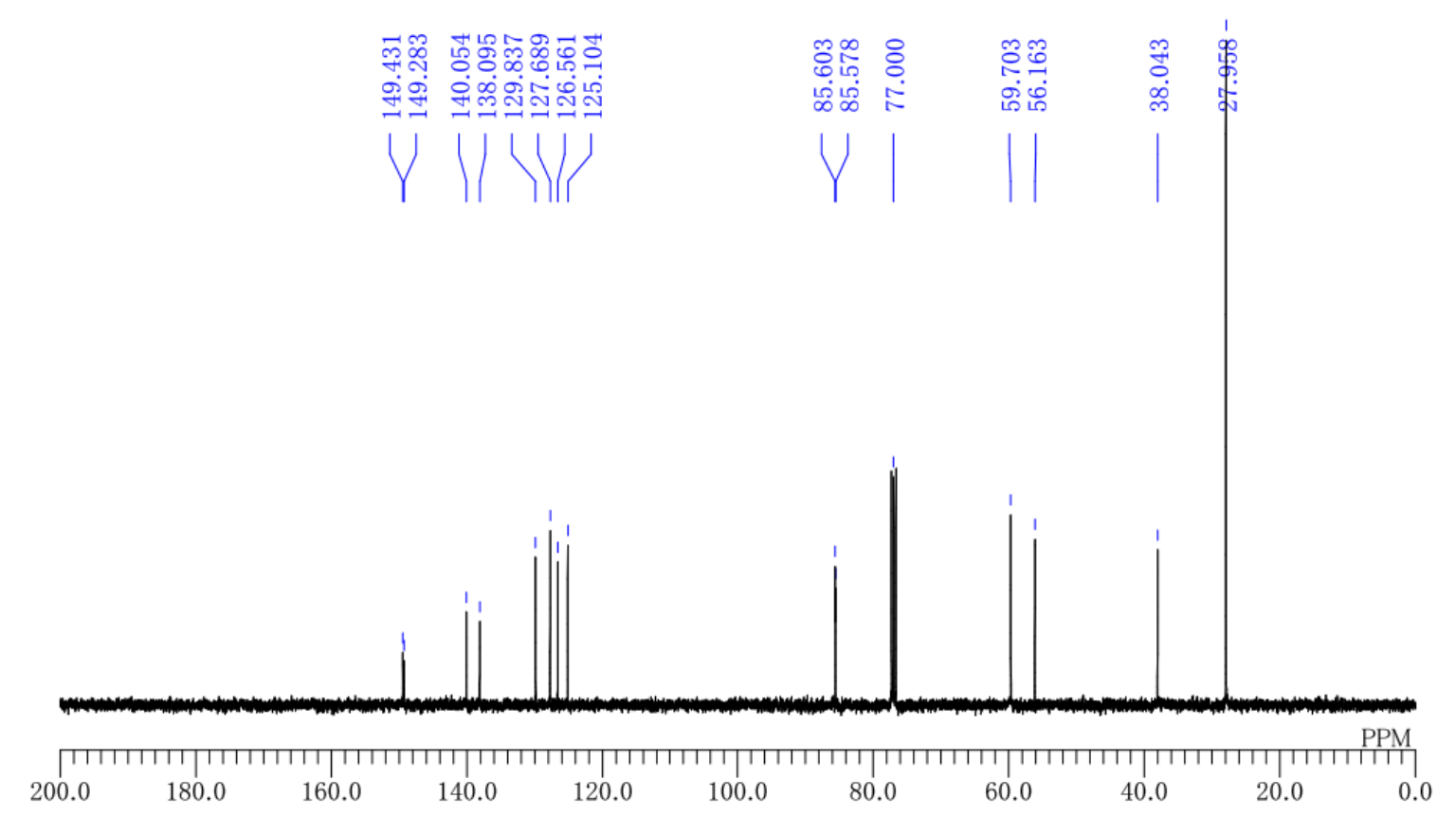


di-tert-butyl $\left(3 \mathrm{a} R^{*}, 8 \mathrm{~b} S^{*}\right)$-6-bromo-inda[1,2-c][1,2,5]thiadiazolidine-1,3-dicarboxylate 2,2-dioxide (5r)<smiles>CC(C)(C)OC(=O)c1ccccc1N1C2Cc3cc(Br)ccc3C2N(N(C(=O)OC(C)(C)C)C(=O)c2ccccc2)S1(=O)=O</smiles>

${ }^{1} \mathrm{H}$ NMR $\left(400 \mathrm{MHz}, \mathrm{CDCl}_{3}\right)$

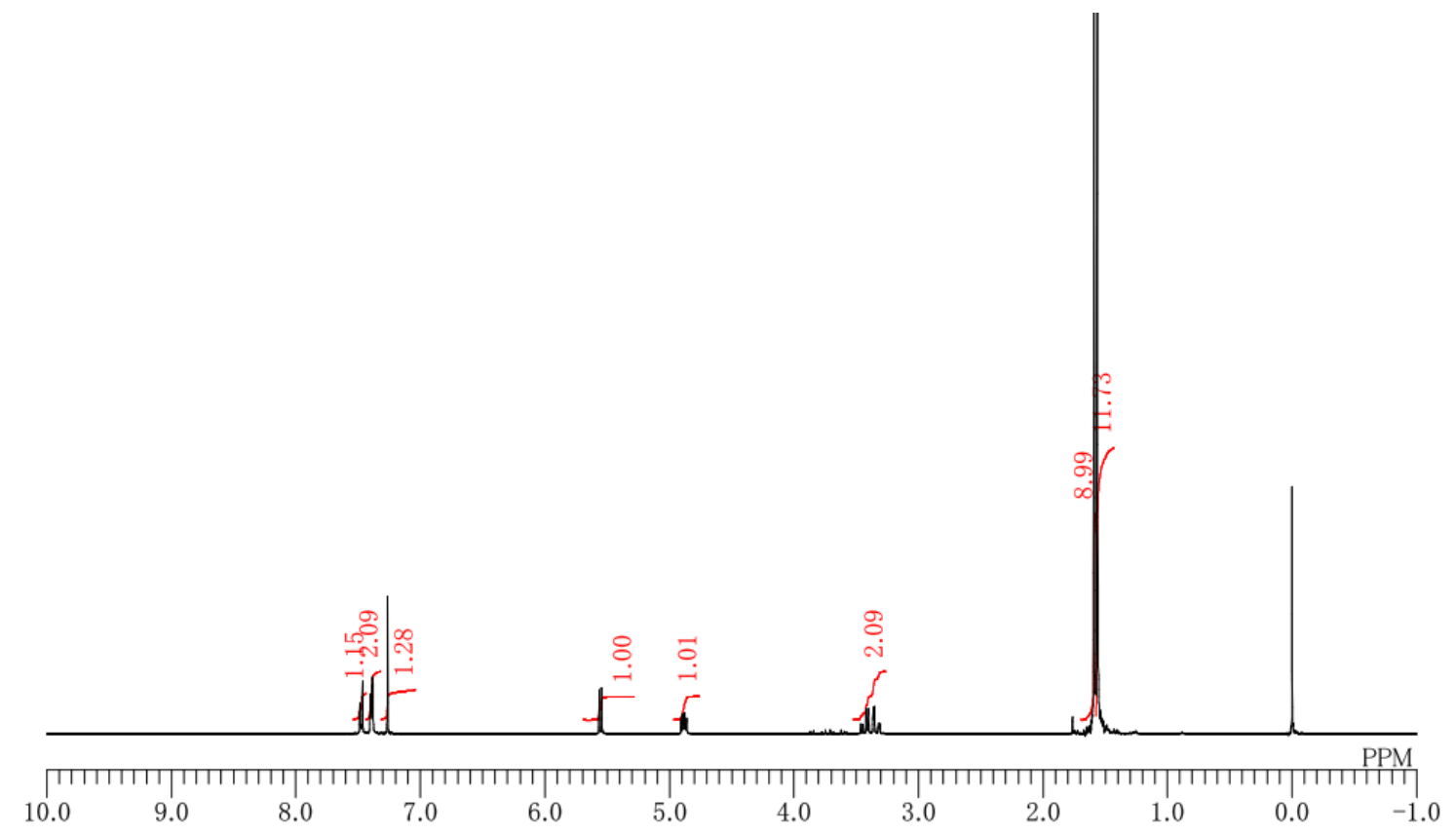

${ }^{13} \mathrm{C}$ NMR $\left(100 \mathrm{MHz}, \mathrm{CDCl}_{3}\right)$
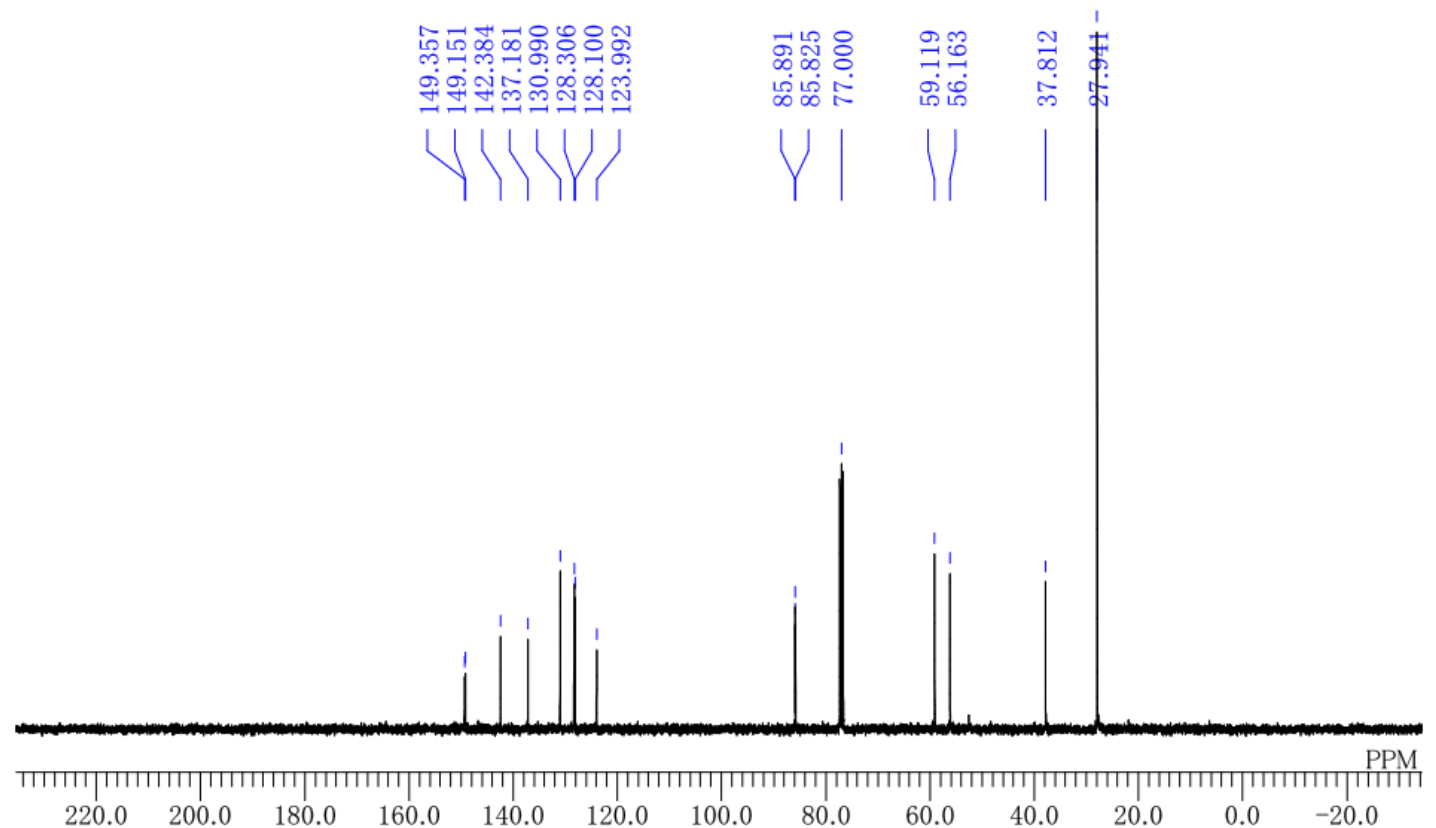
di-tert-butyl $\left(3 \mathrm{a} R^{*}, 9 \mathrm{~b} S^{*}\right)-4,5-d i h y d r o n a p h t h a[1,2-c][1,2,5]$ thiadiazolidine-1,3-dicarboxylate 2,2dioxide (5s)

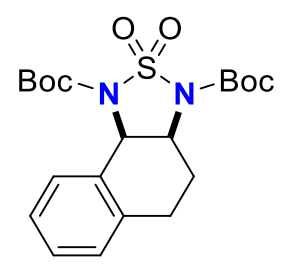

${ }^{1} \mathrm{H}$ NMR $\left(400 \mathrm{MHz}, \mathrm{CDCl}_{3}\right)$

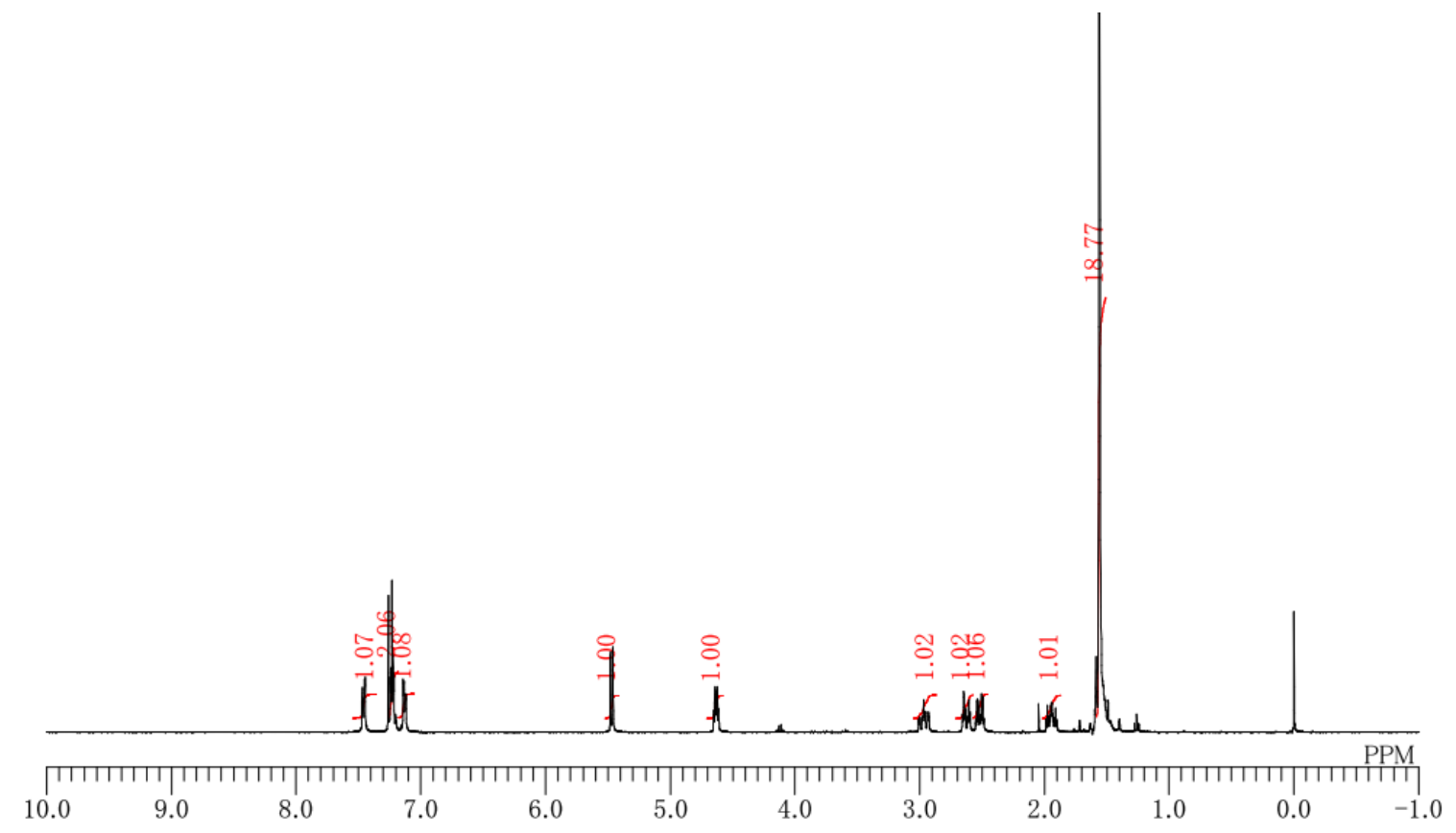

${ }^{13} \mathrm{C}$ NMR $\left(100 \mathrm{MHz}, \mathrm{CDCl}_{3}\right)$

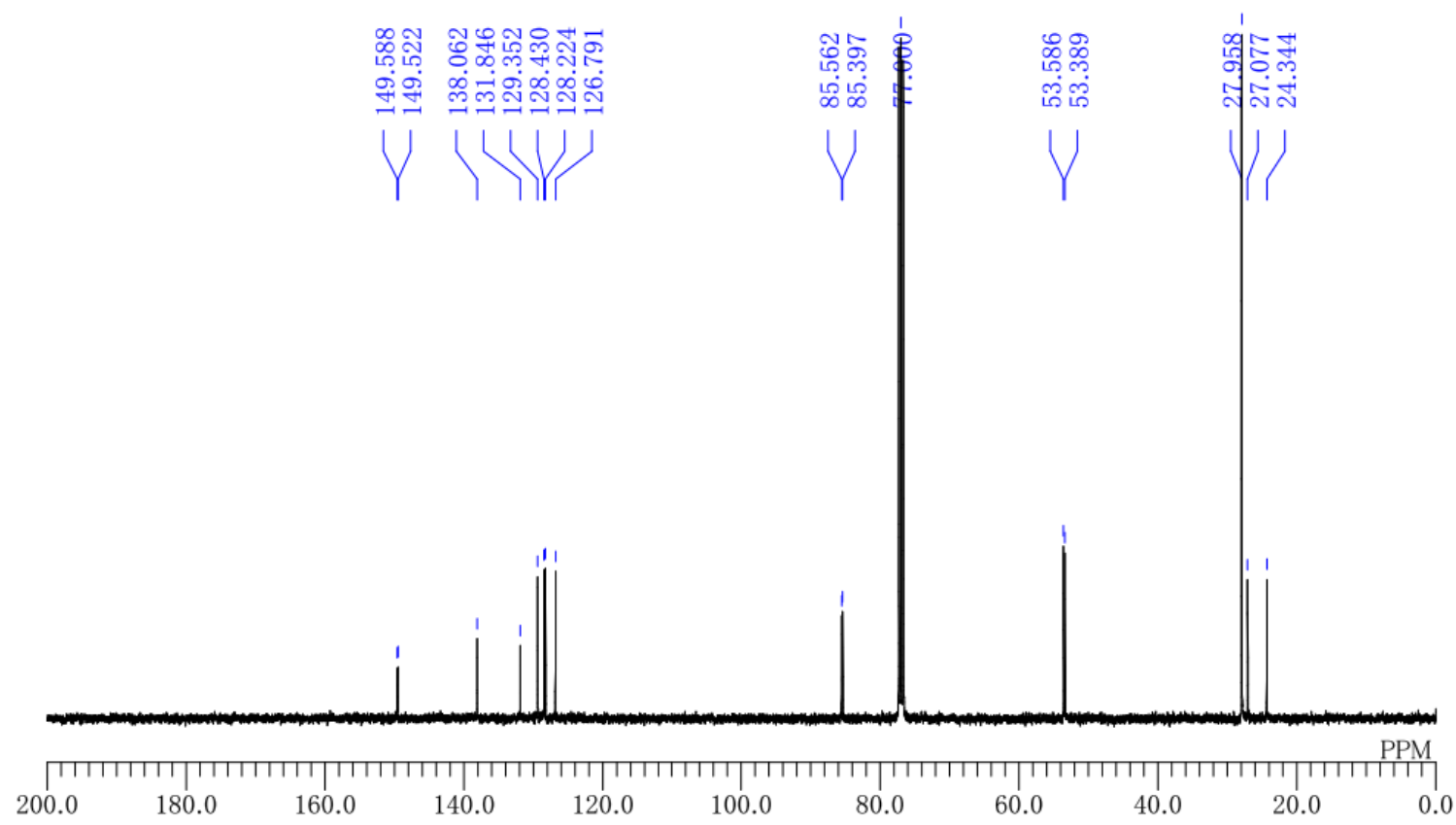

di-tert-butyl (3aS,9bR)-7-methoxy-3a,4,5,9b-tetrahydronaphtho[1,2-c][1,2,5]thiadiazole-1,3dicarboxylate 2,2-dioxide (5t) 


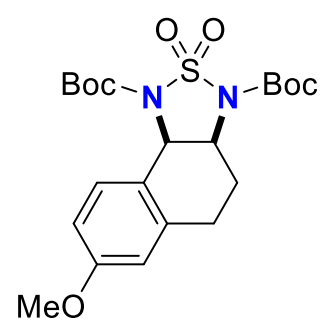

${ }^{1} \mathrm{H}$ NMR (400 MHz, $\left.\mathrm{CDCl}_{3}\right)$

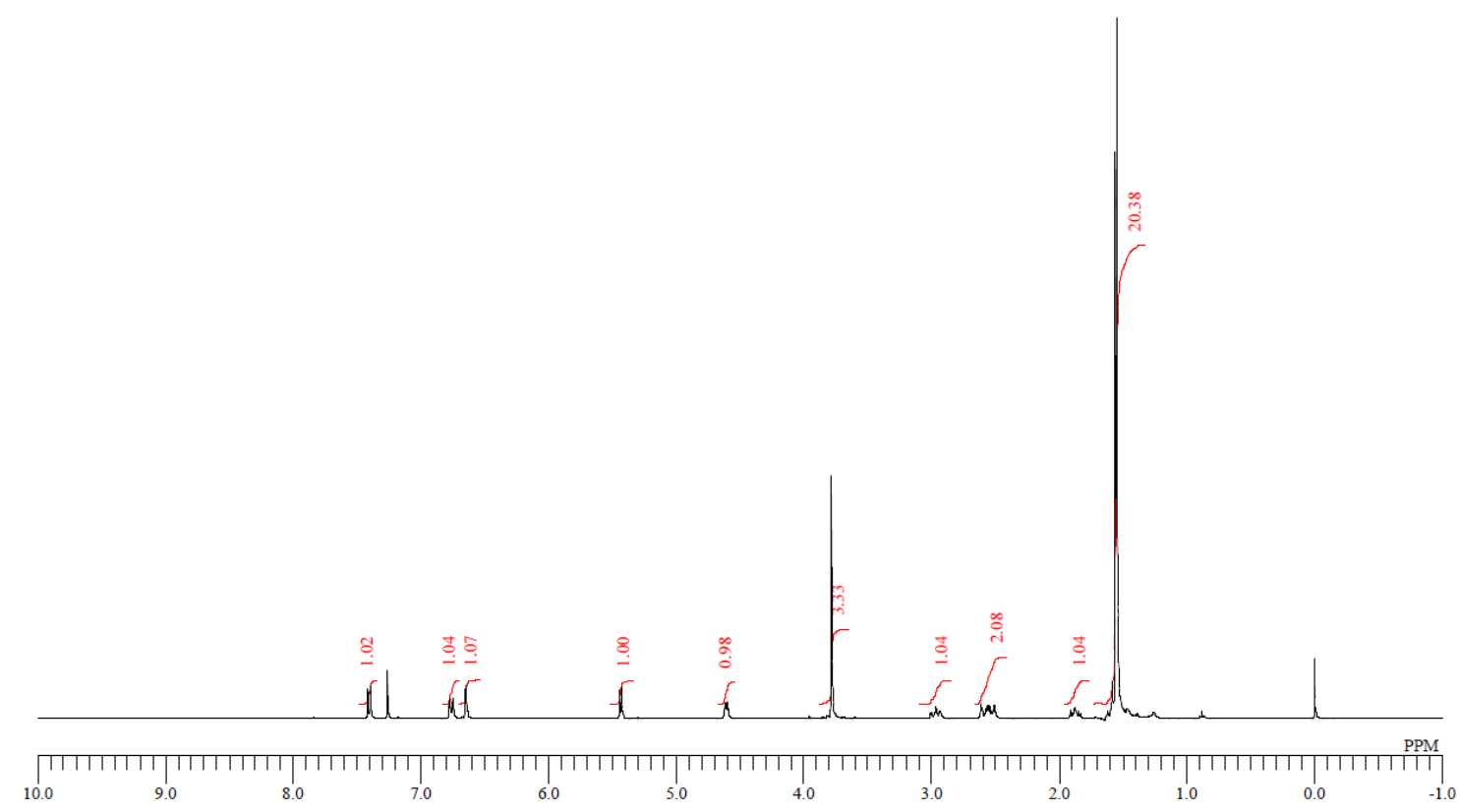

${ }^{13} \mathrm{C} \mathrm{NMR}\left(100 \mathrm{MHz}, \mathrm{CDCl}_{3}\right)$
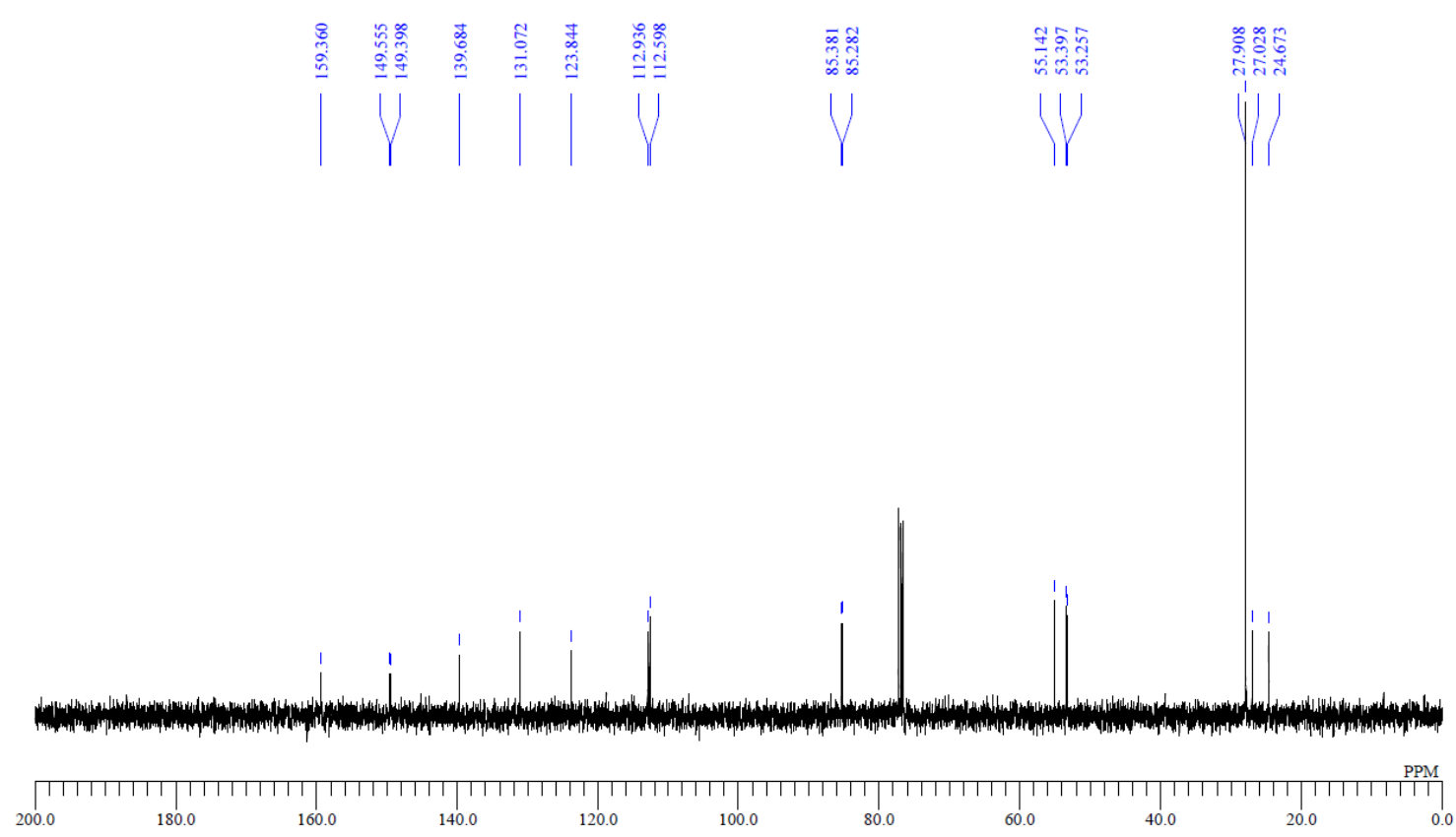

tri-tert-butyl $\left(3 \mathrm{a} R^{*}, 8 \mathrm{~b} S^{*}\right)$-indo[2,3-c][1,2,5]thiadiazolidine-1,3,4-tricarboxylate 2,2-dioxide (5u) 


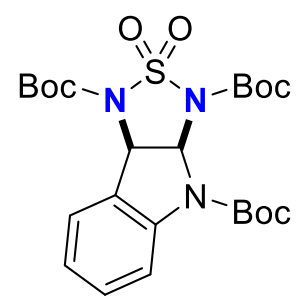

${ }^{1} \mathrm{H}$ NMR $\left(400 \mathrm{MHz}, \mathrm{CDCl}_{3}\right)$

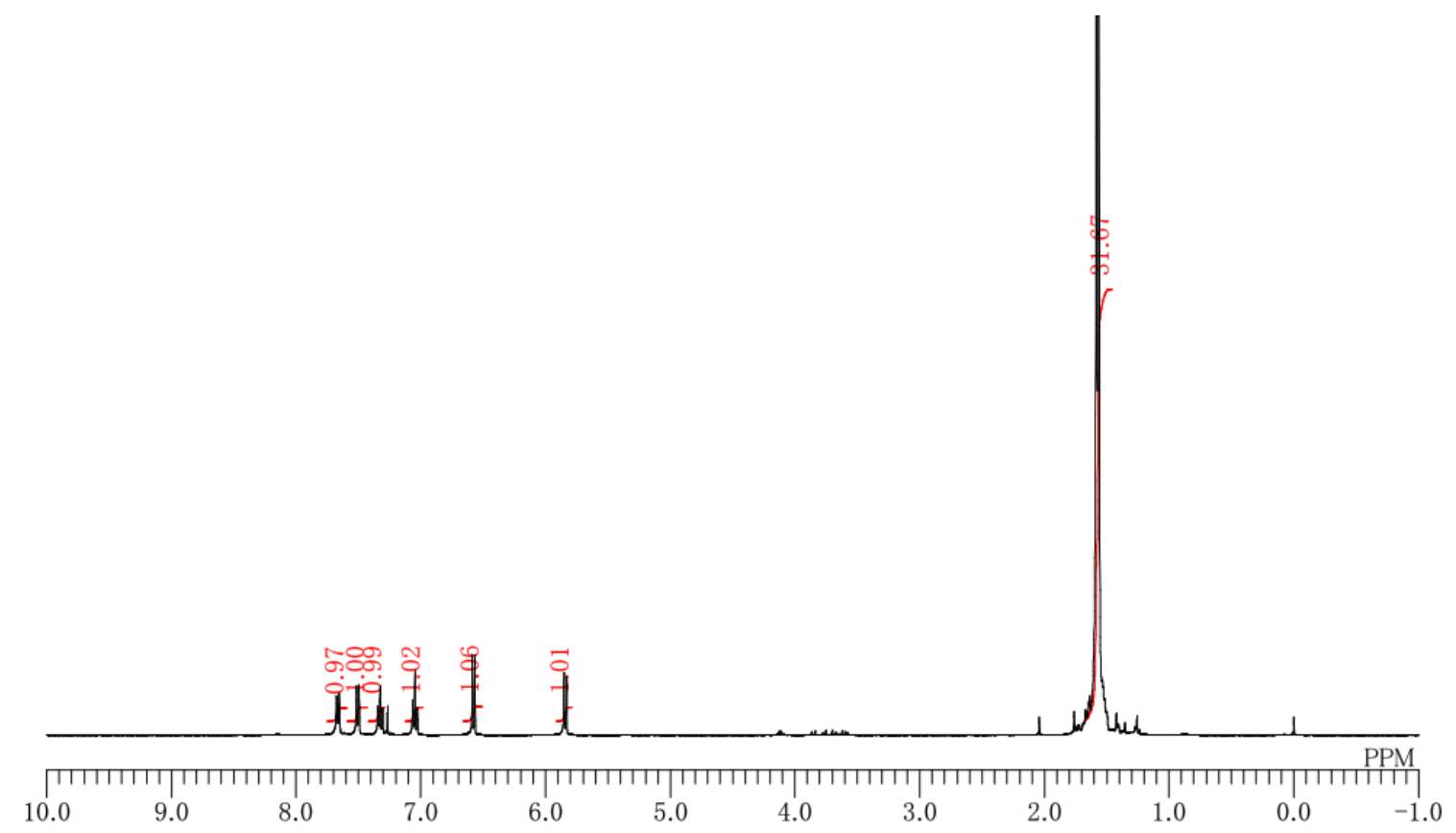

${ }^{13} \mathrm{C}$ NMR $\left(100 \mathrm{MHz}, \mathrm{CDCl}_{3}\right)$

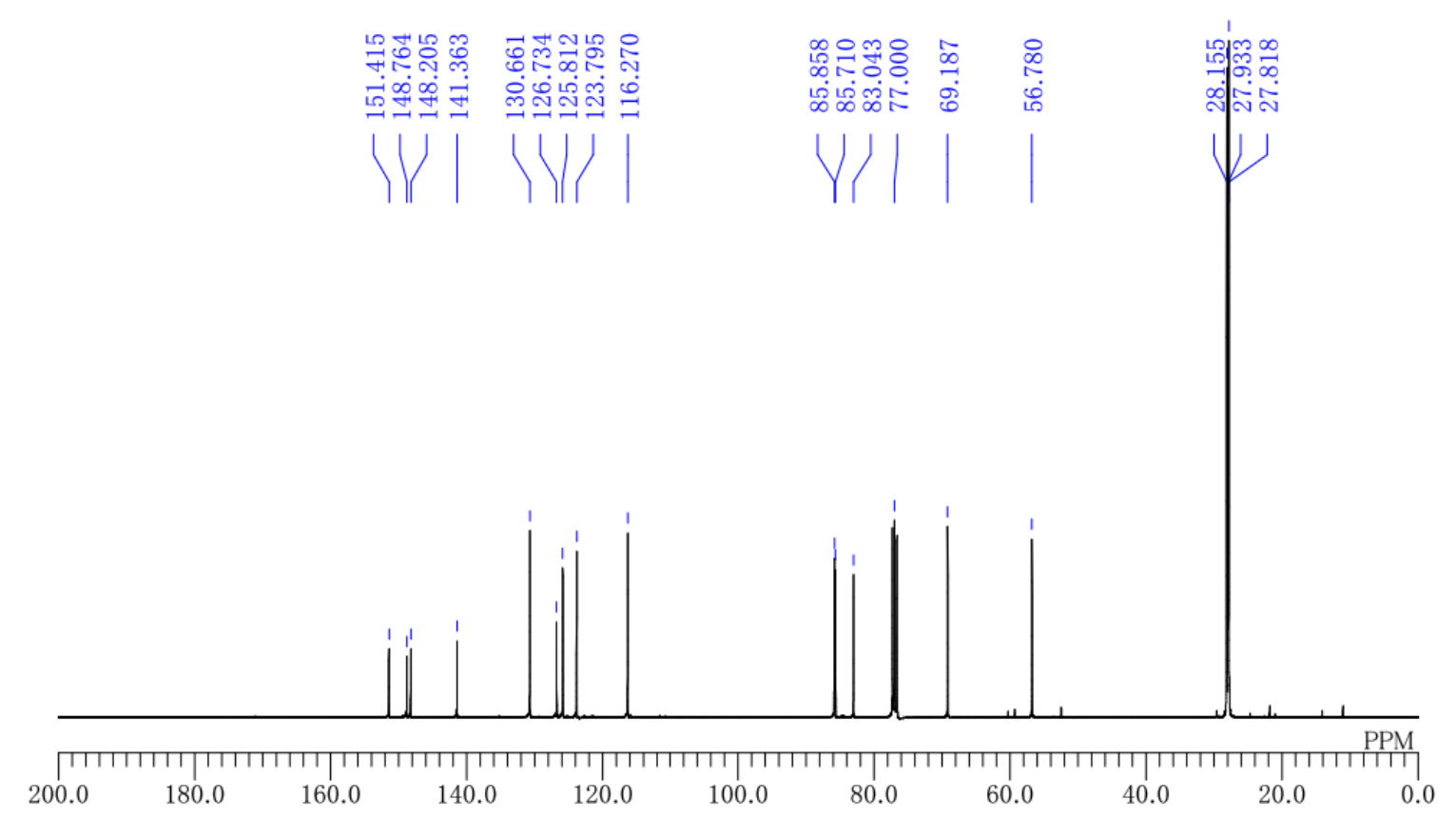

tri-tert-butyl (3aR,8bR)-7-methoxy-3a,8b-dihydro-1H-[1,2,5] thiadiazolo[3,4-b]indole-1,3,4tricarboxylate 2,2-dioxide (5v) 


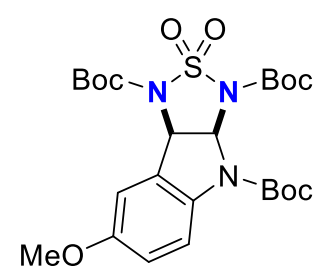

${ }^{1} \mathrm{H}$ NMR (400 MHz, $\left.\mathrm{CDCl}_{3}\right)$

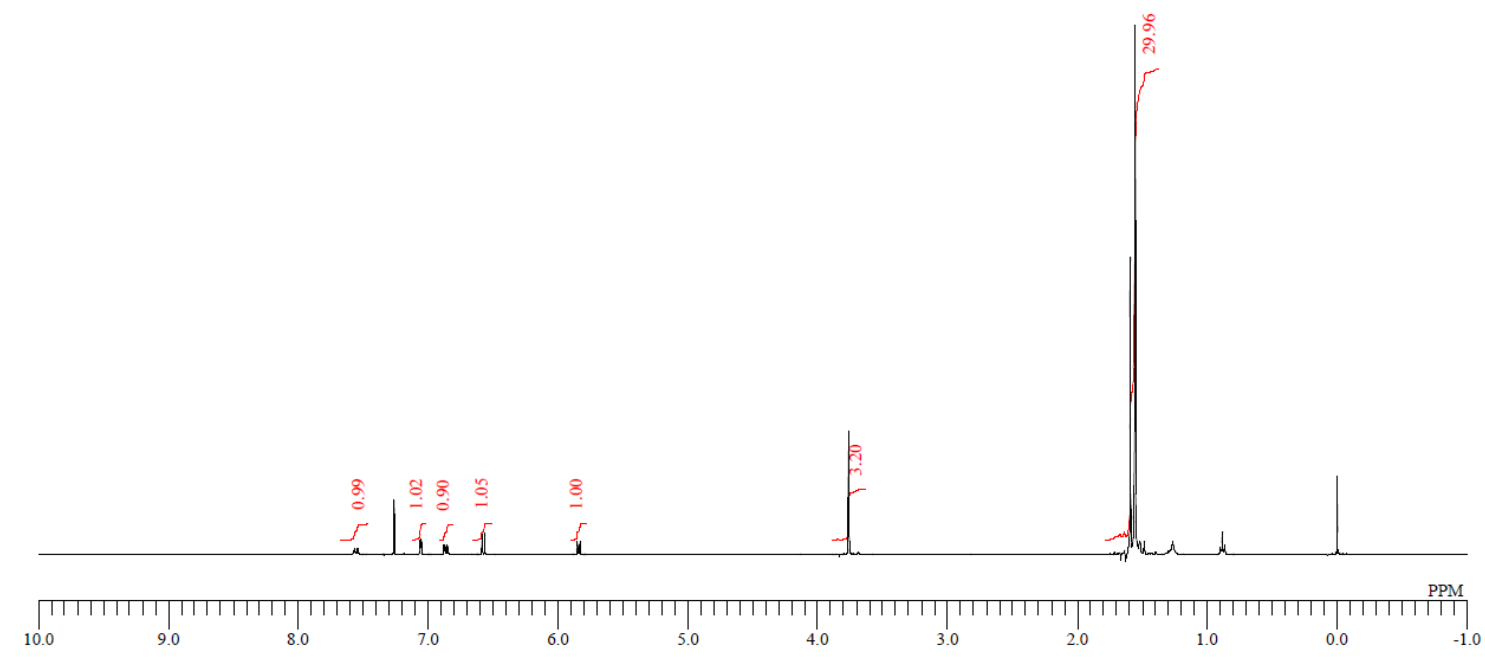

${ }^{13} \mathrm{C}$ NMR $\left(100 \mathrm{MHz}, \mathrm{CDCl}_{3}\right)$
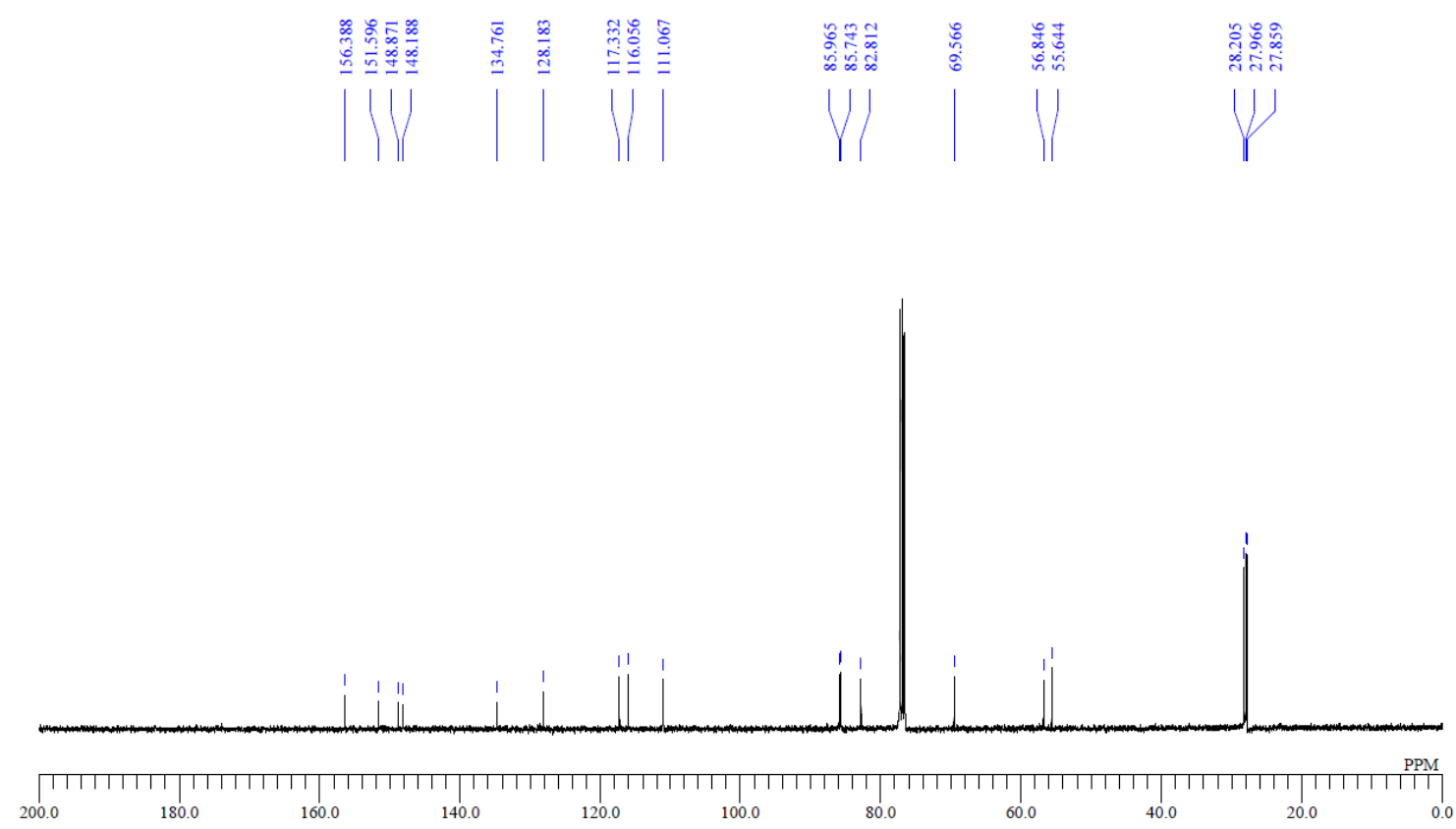

di-tert-butyl $\left(3 R^{*}, 4 R^{*}\right)$-3,4-dipropyl-1,2,5-thiadiazolidine-2,5-dicarboxylate 1,1-dioxide (5f) 


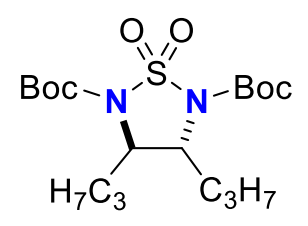

${ }^{1} \mathrm{H}$ NMR (400 MHz, $\mathrm{CDCl}_{3}$ )

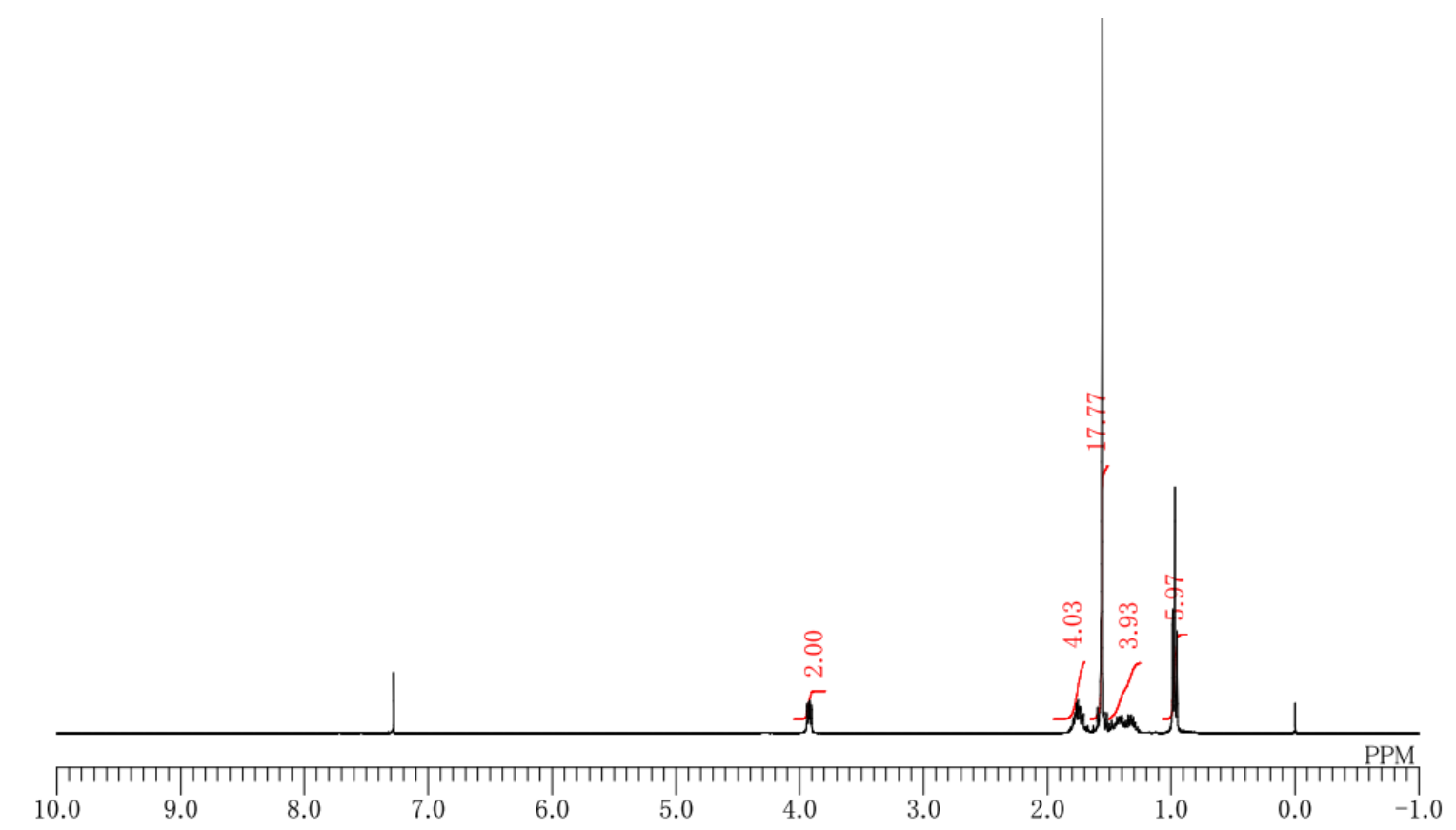

${ }^{13} \mathrm{C}$ NMR (100 MHz, $\mathrm{CDCl}_{3}$ )

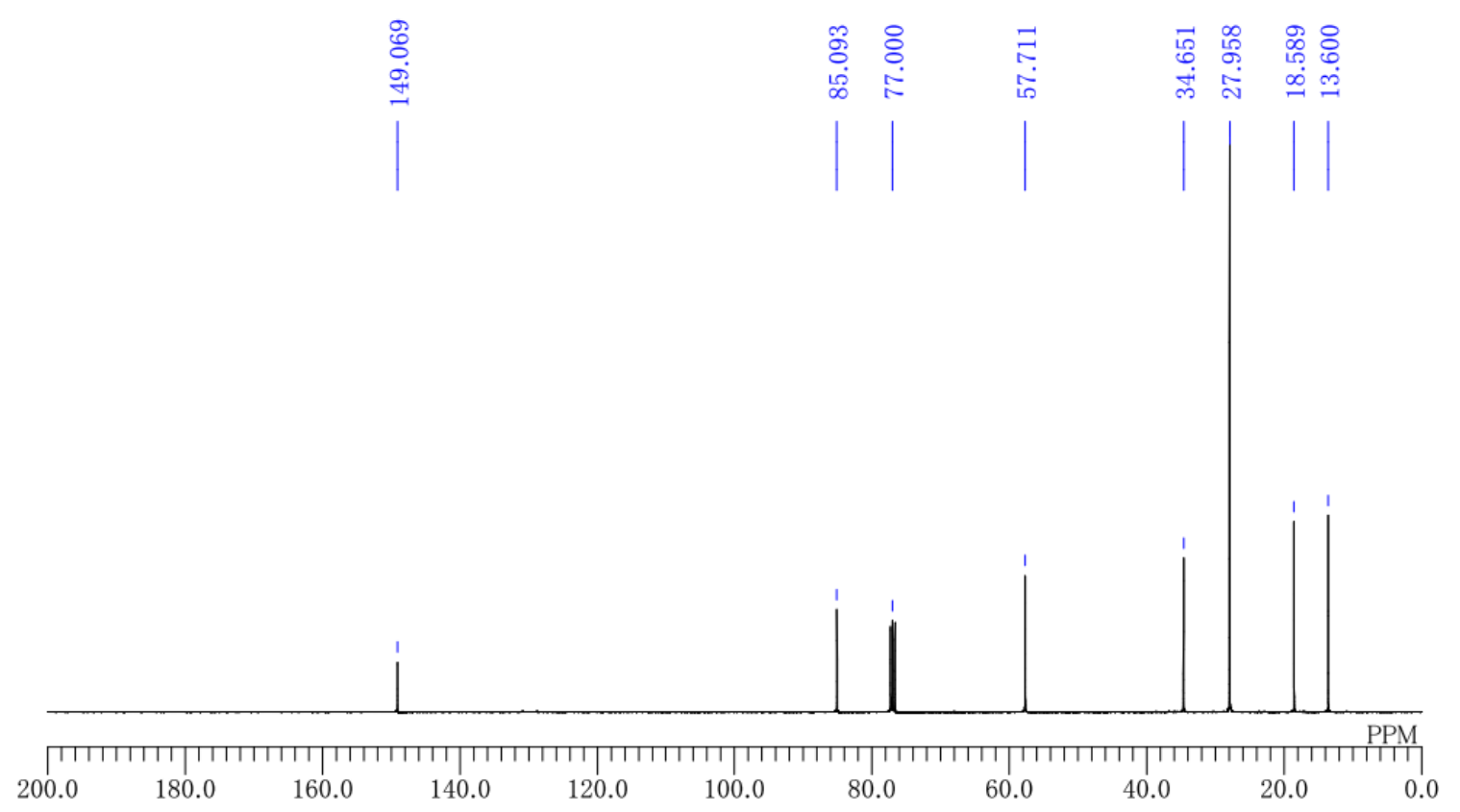

di-tert-butyl $\left(3 R^{*}, 4 S^{*}\right)$-3,4-dipropyl-1,2,5-thiadiazolidine-2,5-dicarboxylate 1,1-dioxide (5g) 


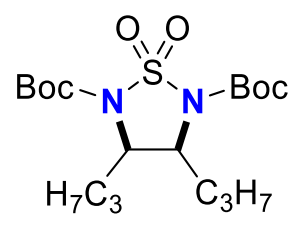

${ }^{1} \mathrm{H}$ NMR (400 MHz, $\mathrm{CDCl}_{3}$ )

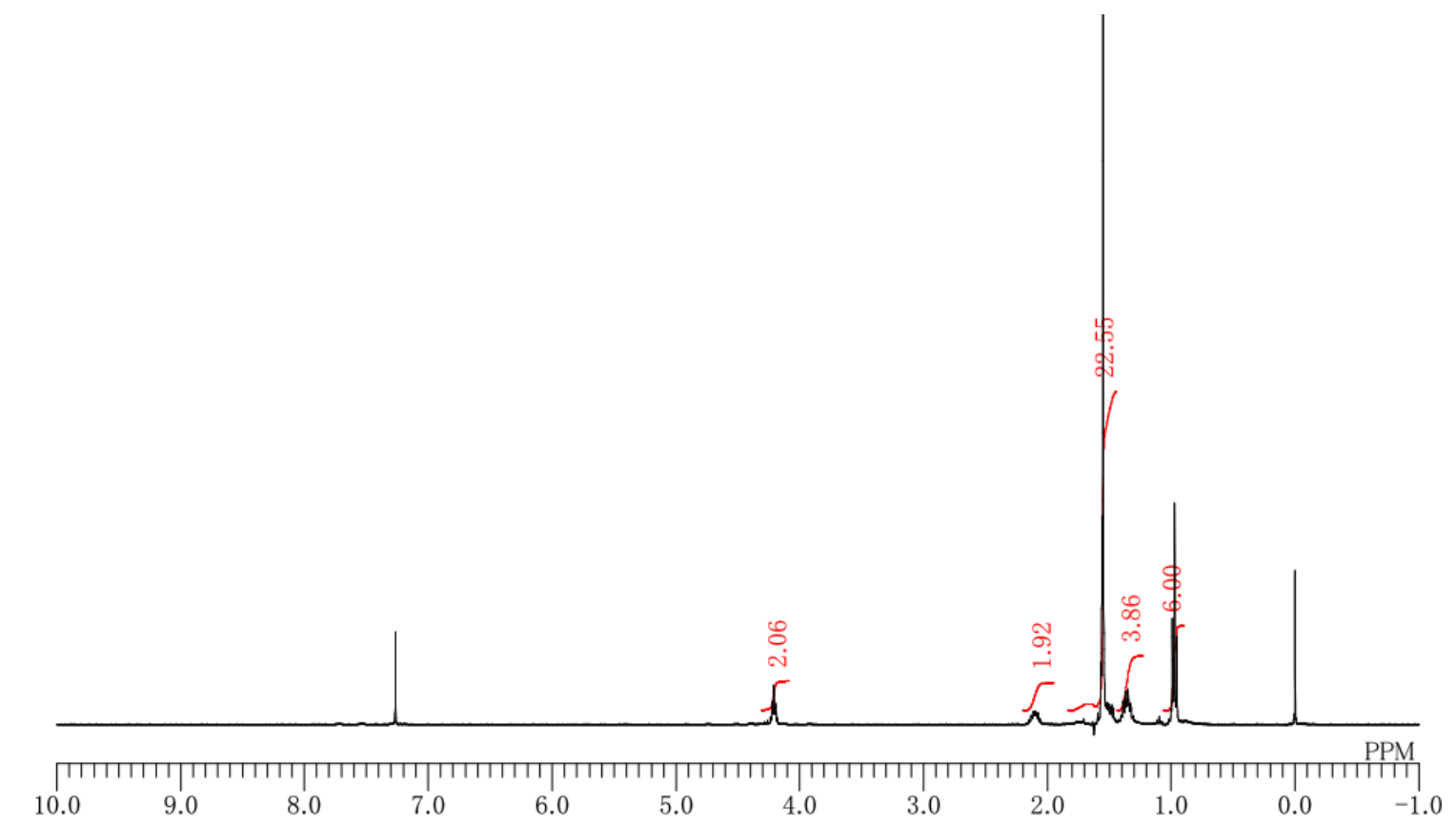

${ }^{13} \mathrm{C}$ NMR (100 MHz, $\mathrm{CDCl}_{3}$ )

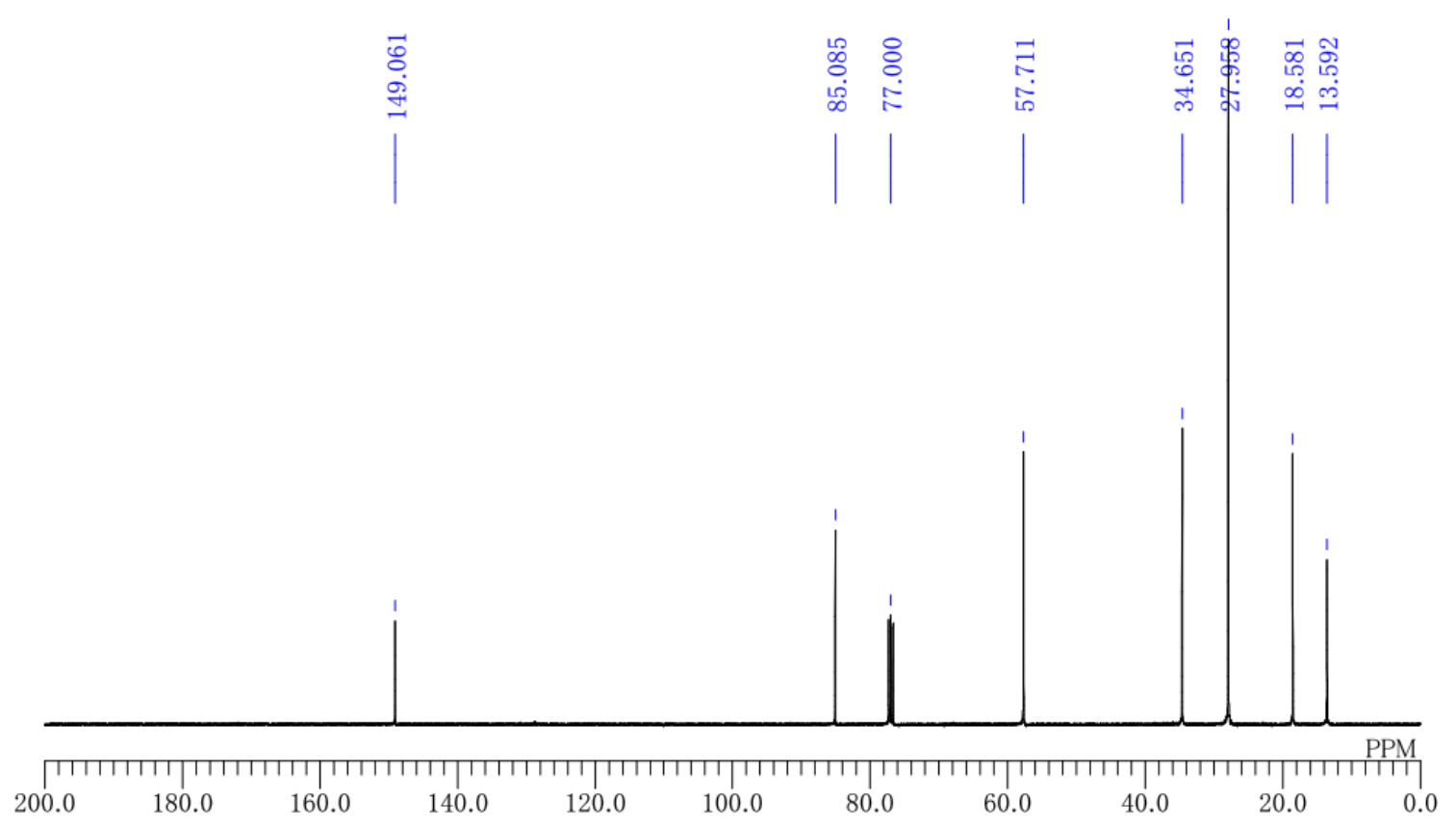

di-tert-butyl (3R,4R)-3-methyl-4-propyl-1,2,5-thiadiazolidine-2,5-dicarboxylate 1,1-dioxide-benzene (5j) 


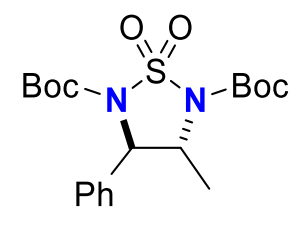

${ }^{1} \mathrm{H}$ NMR (400 MHz, $\mathrm{CDCl}_{3}$ )

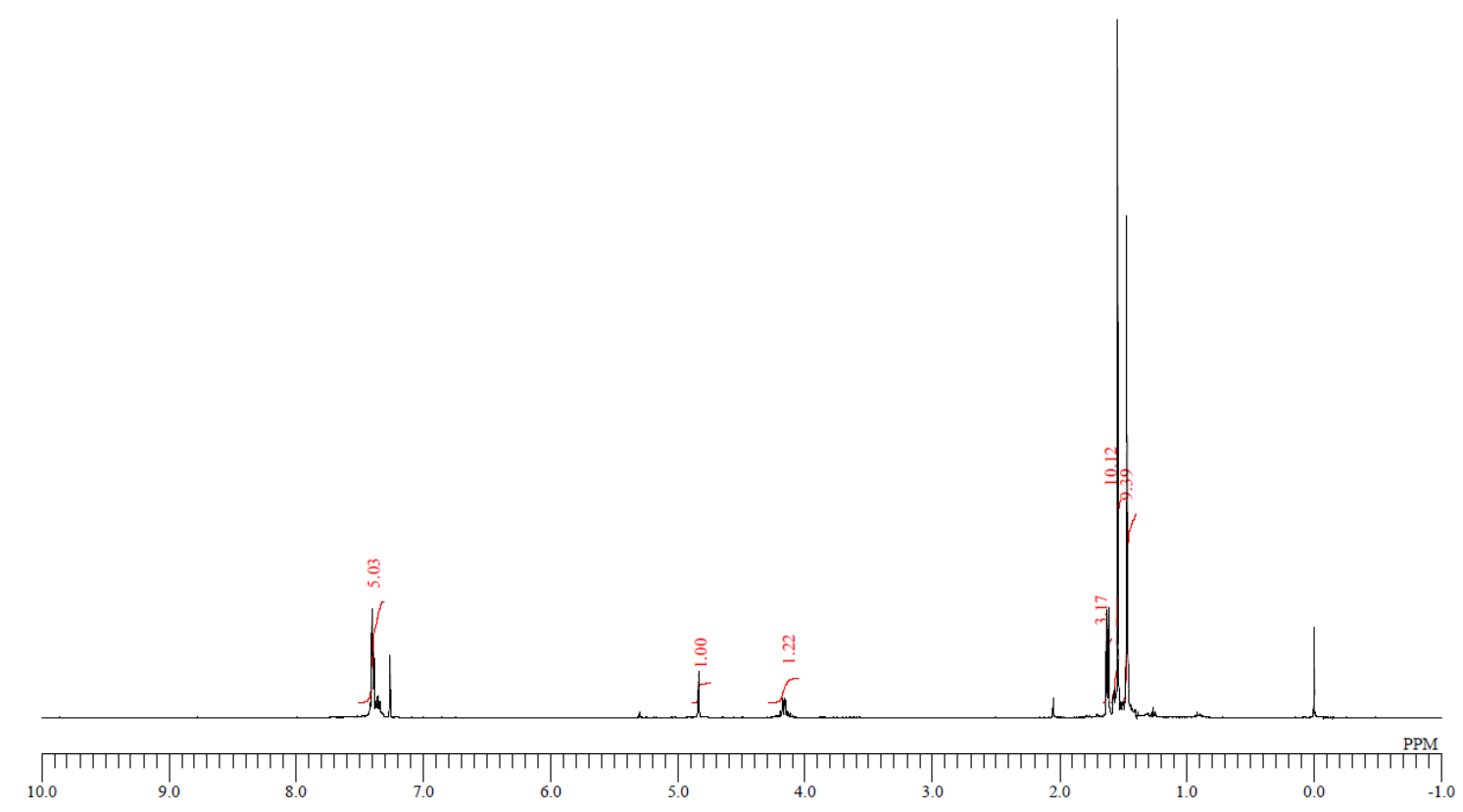

${ }^{13} \mathrm{C}$ NMR $\left(100 \mathrm{MHz}, \mathrm{CDCl}_{3}\right)$
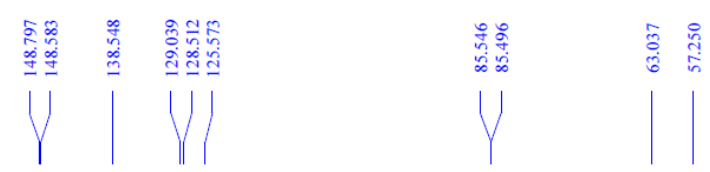

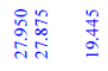
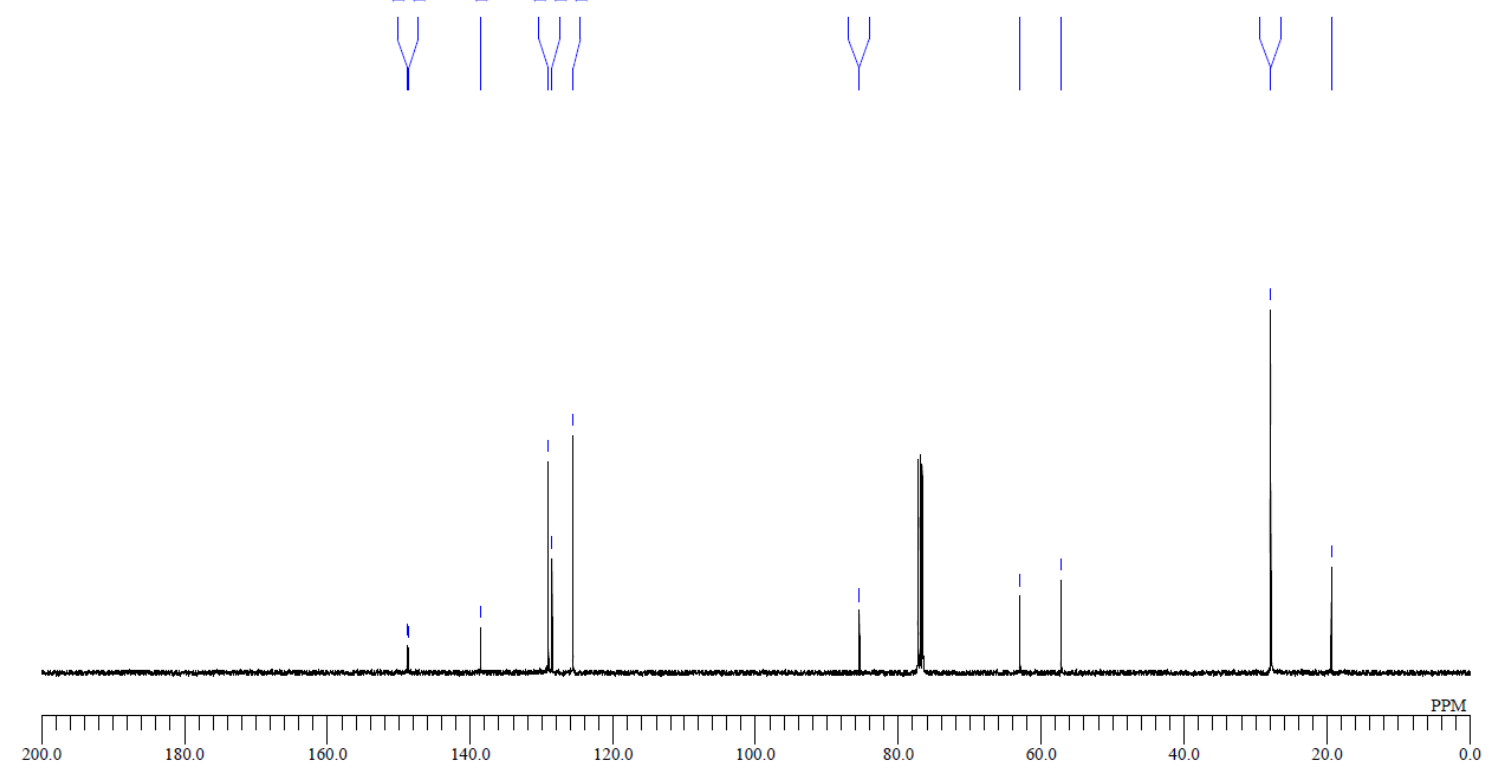

tert-butyl ((4R*, $\left.\left.5 S^{*}\right)-5-((2-n i t r o p h e n y l) s u l f o n a m i d o) o c t a n-4-y l\right)$ carbamate (4fa) 


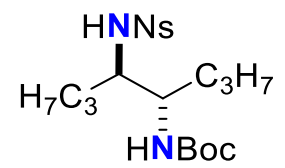

${ }^{1} \mathrm{H}$ NMR (400 MHz, $\mathrm{CDCl}_{3}$ )

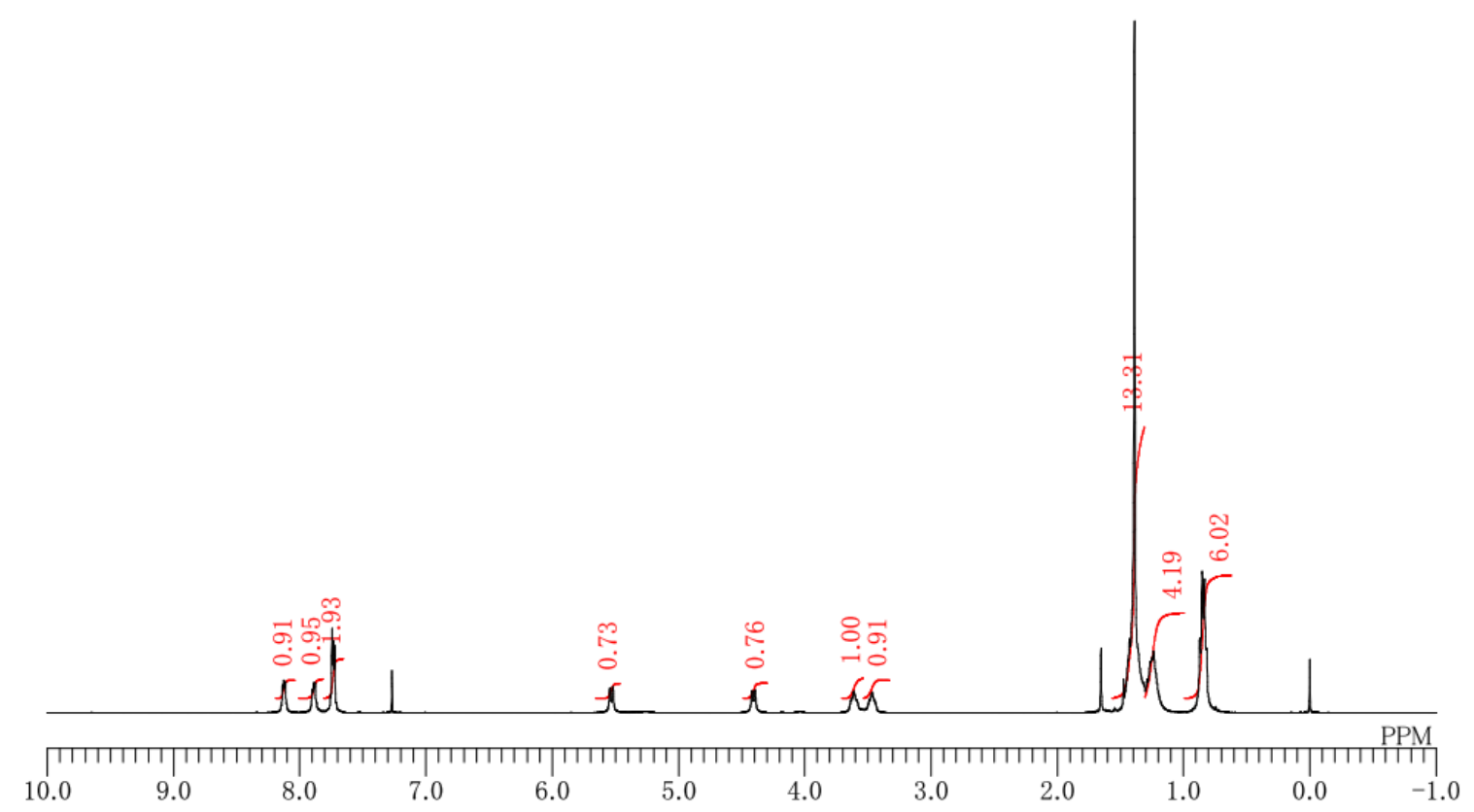

${ }^{13} \mathrm{C}$ NMR (100 MHz, $\mathrm{CDCl}_{3}$ )

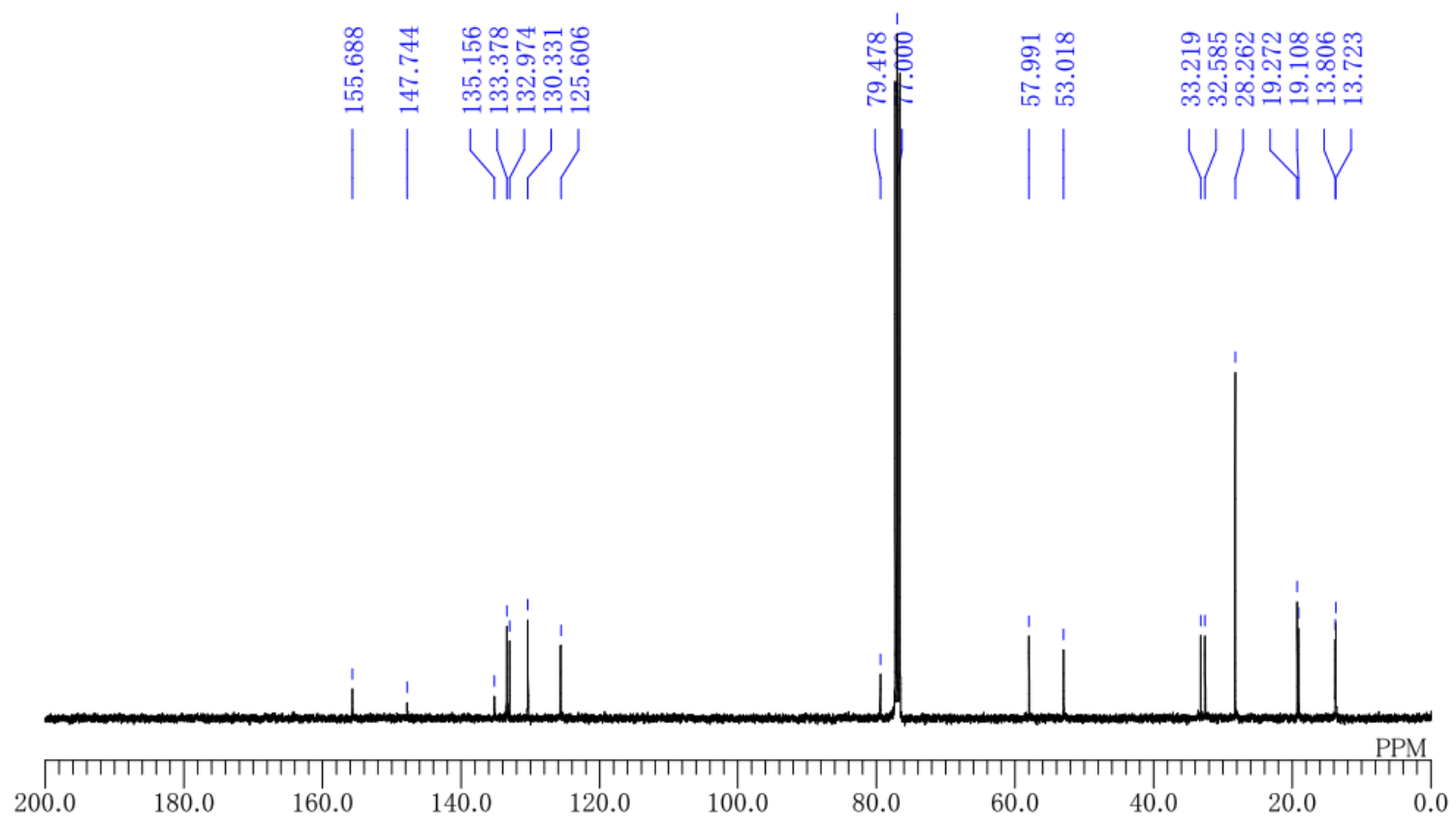

tert-butyl $\left(\left(4 \mathrm{R}^{*}, 5 \mathrm{~S} *\right)-5\right.$-aminooctan-4-yl)carbamate $(4 \mathrm{fb})$ 


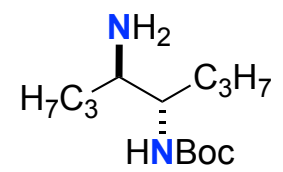

${ }^{1} \mathrm{H}$ NMR $\left(400 \mathrm{MHz}, \mathrm{CDCl}_{3}\right)$

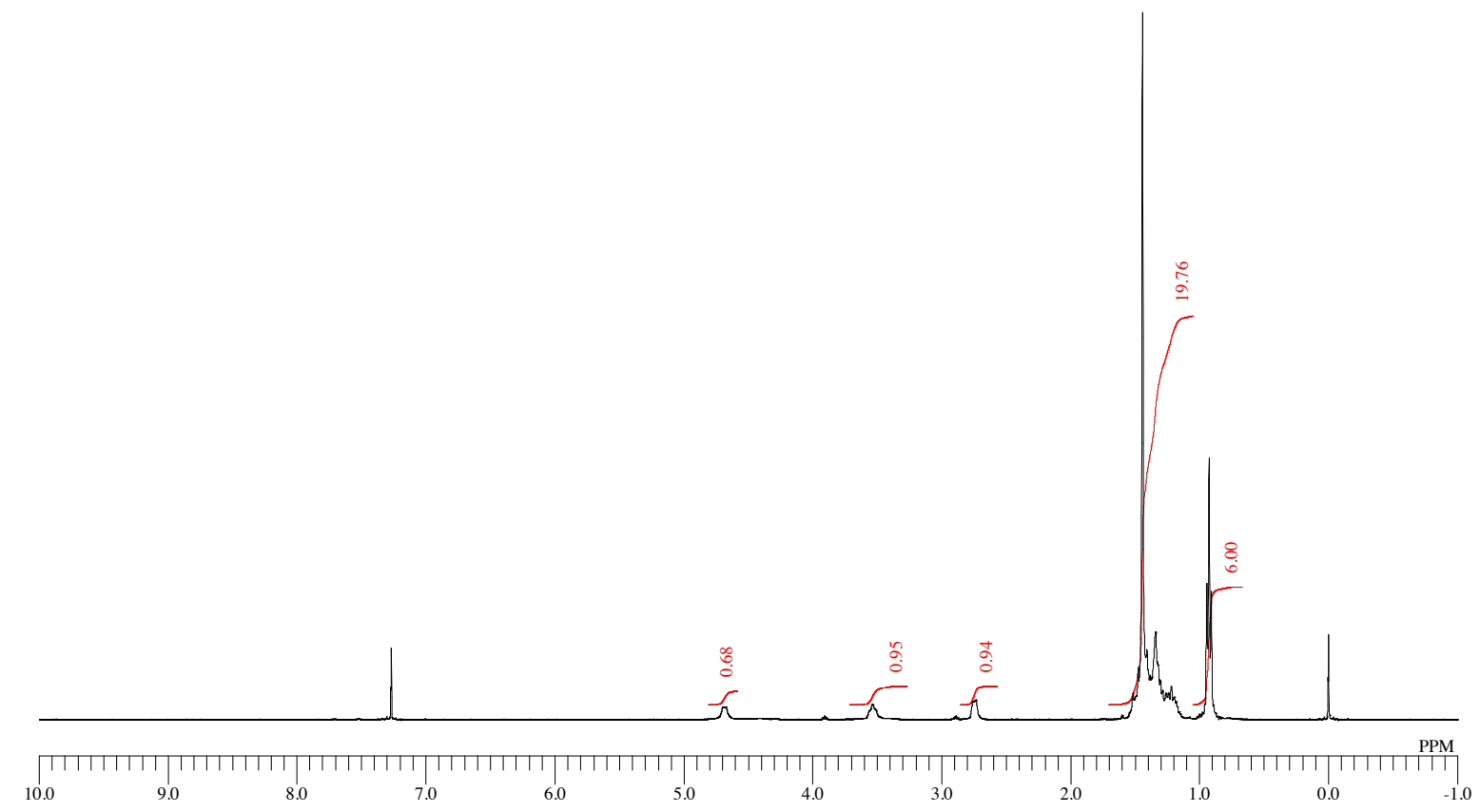

${ }^{13} \mathrm{C}$ NMR $\left(100 \mathrm{MHz}, \mathrm{CDCl}_{3}\right)$

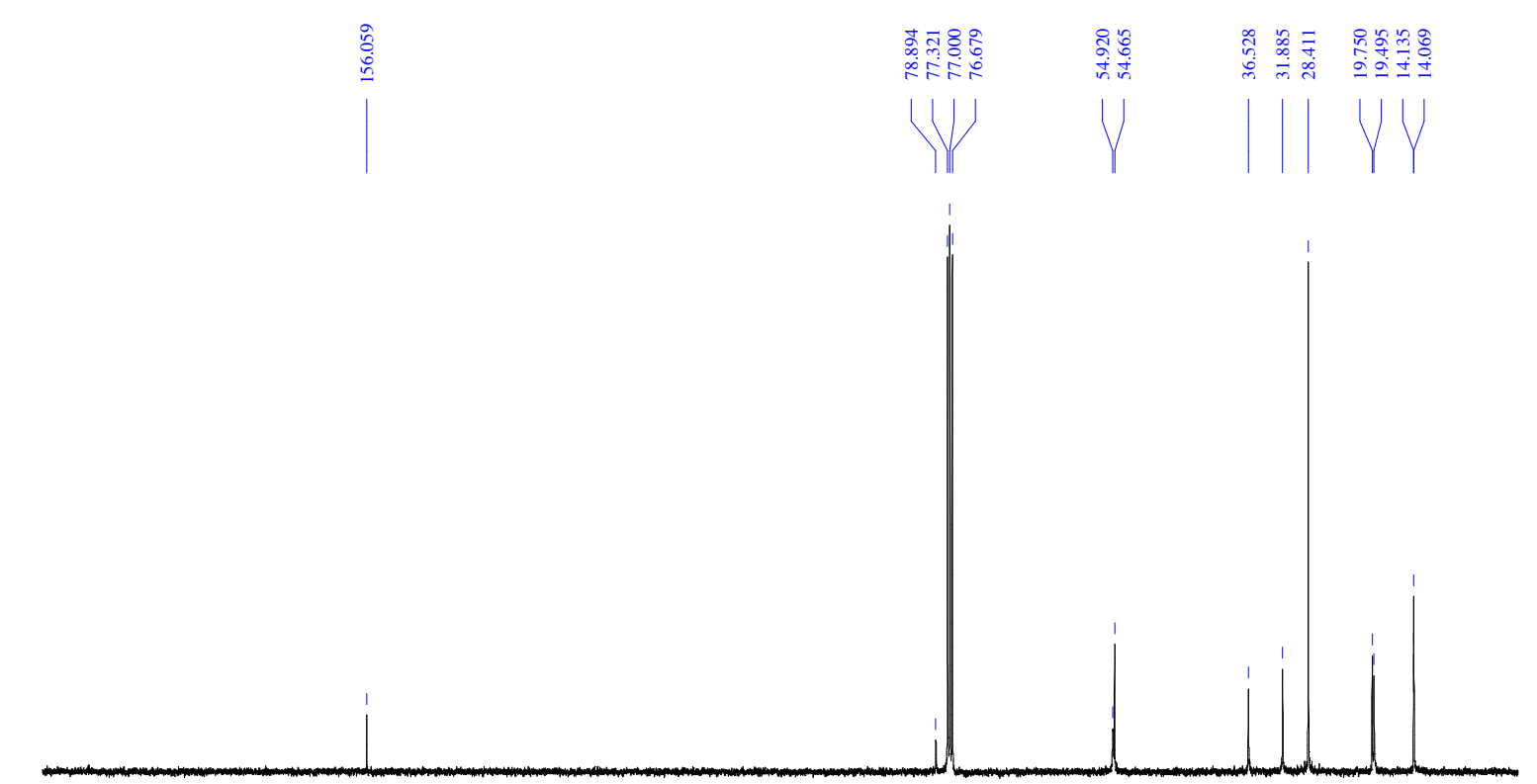

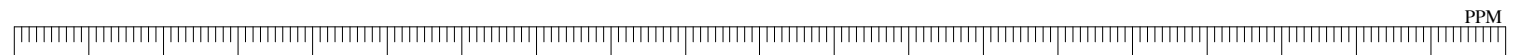

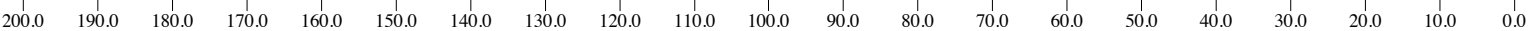

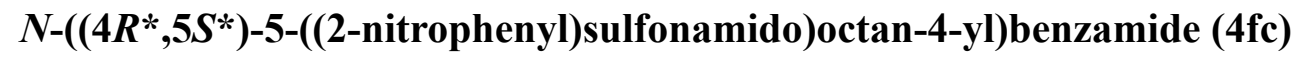




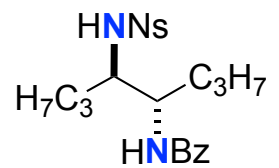

${ }^{1} \mathrm{H}$ NMR $\left(400 \mathrm{MHz}, \mathrm{CDCl}_{3}\right)$

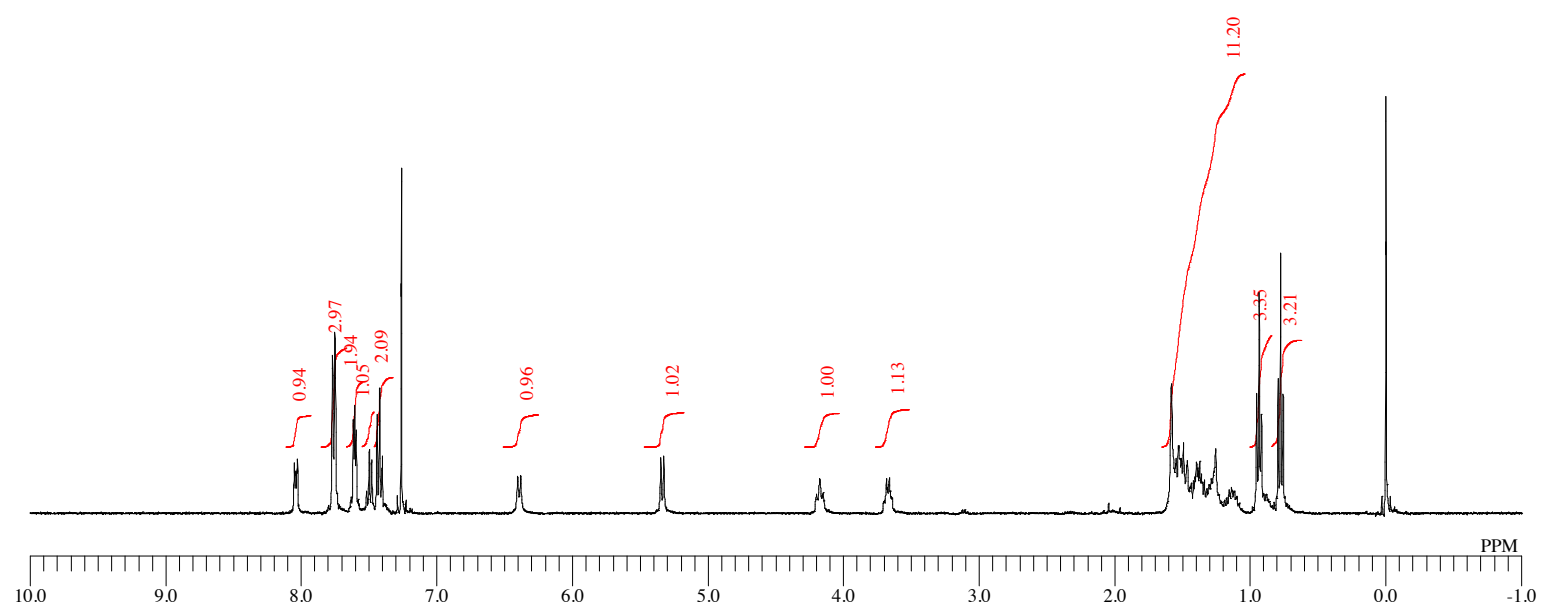

${ }^{13} \mathrm{C} \mathrm{NMR}\left(100 \mathrm{MHz}, \mathrm{CDCl}_{3}\right)$

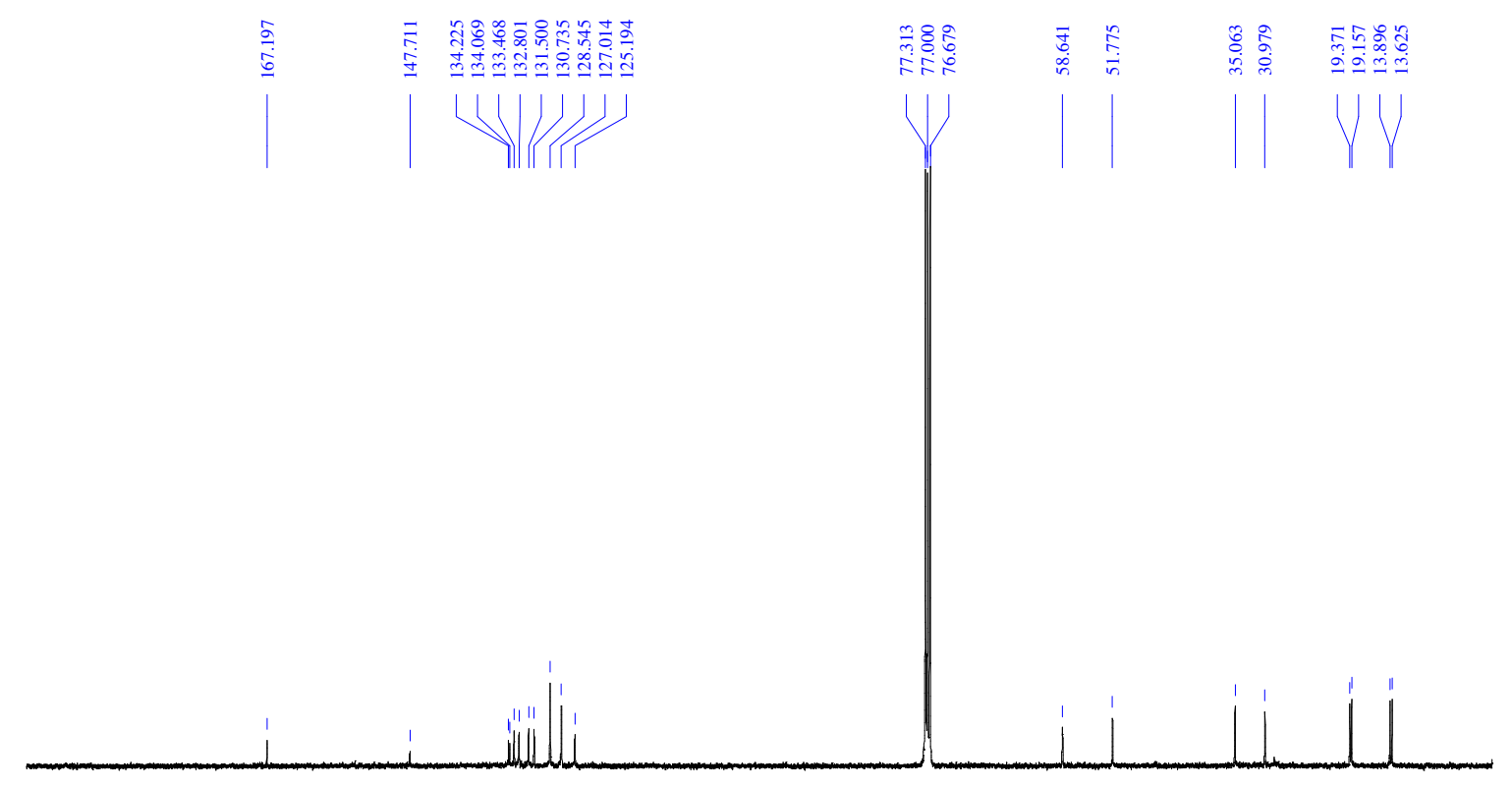

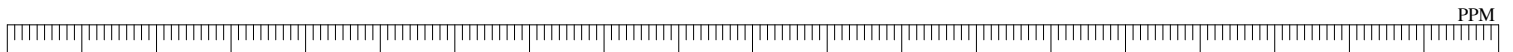

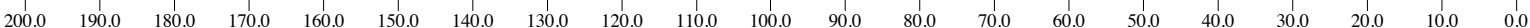


$N, N^{\prime}$-(1-phenylethane-1,2-diyl)bis( $N, N^{\prime}$-dimethyl-2-nitrobenzenesulfonamide) (3ka)<smiles>CN(C)S(C)(=O)(O)CC(c1ccccc1)c1ccccc1</smiles>

${ }^{1} \mathrm{H}$ NMR (400 MHz, $\mathrm{CDCl}_{3}$ )

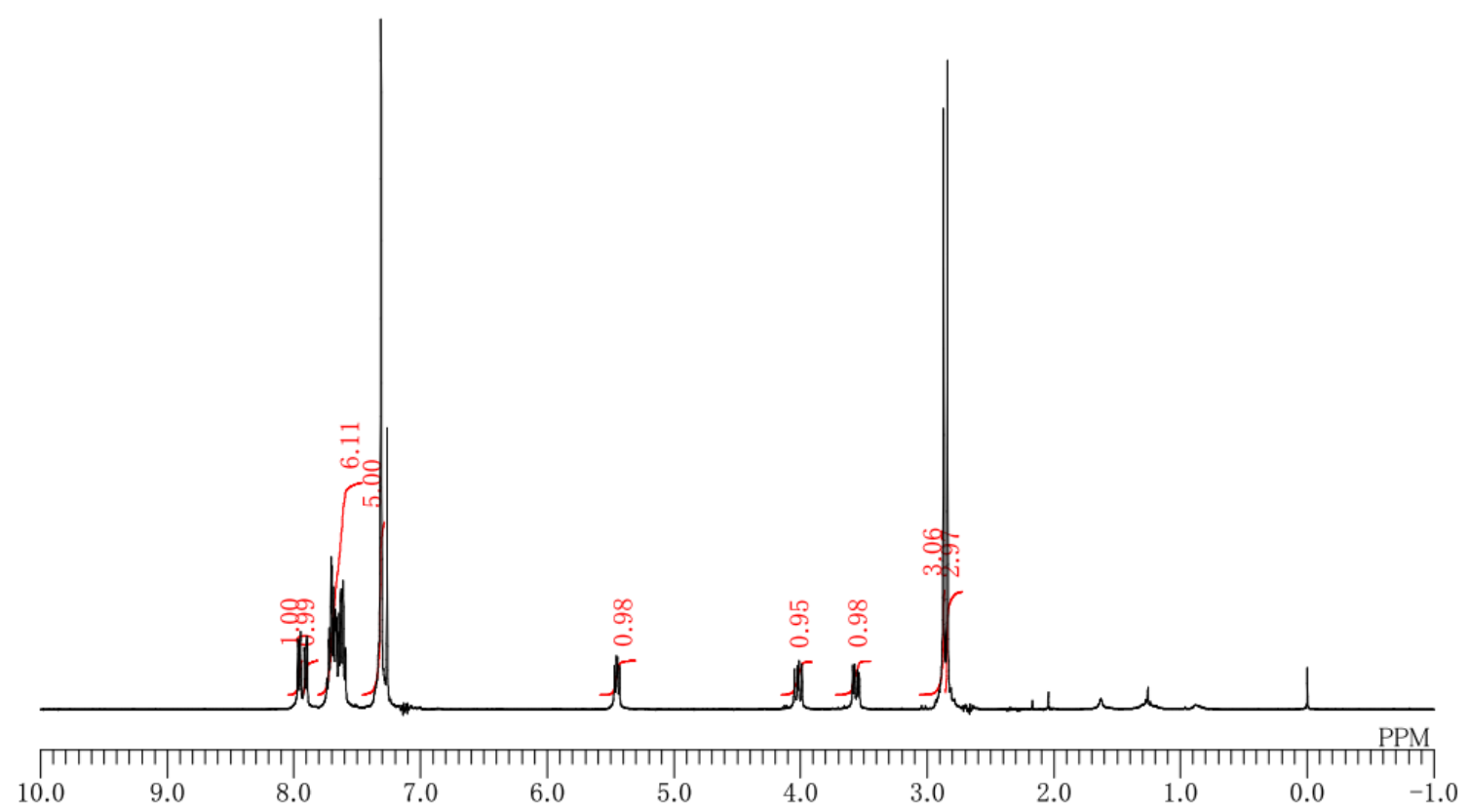

${ }^{13} \mathrm{C}$ NMR $\left(100 \mathrm{MHz}, \mathrm{CDCl}_{3}\right)$

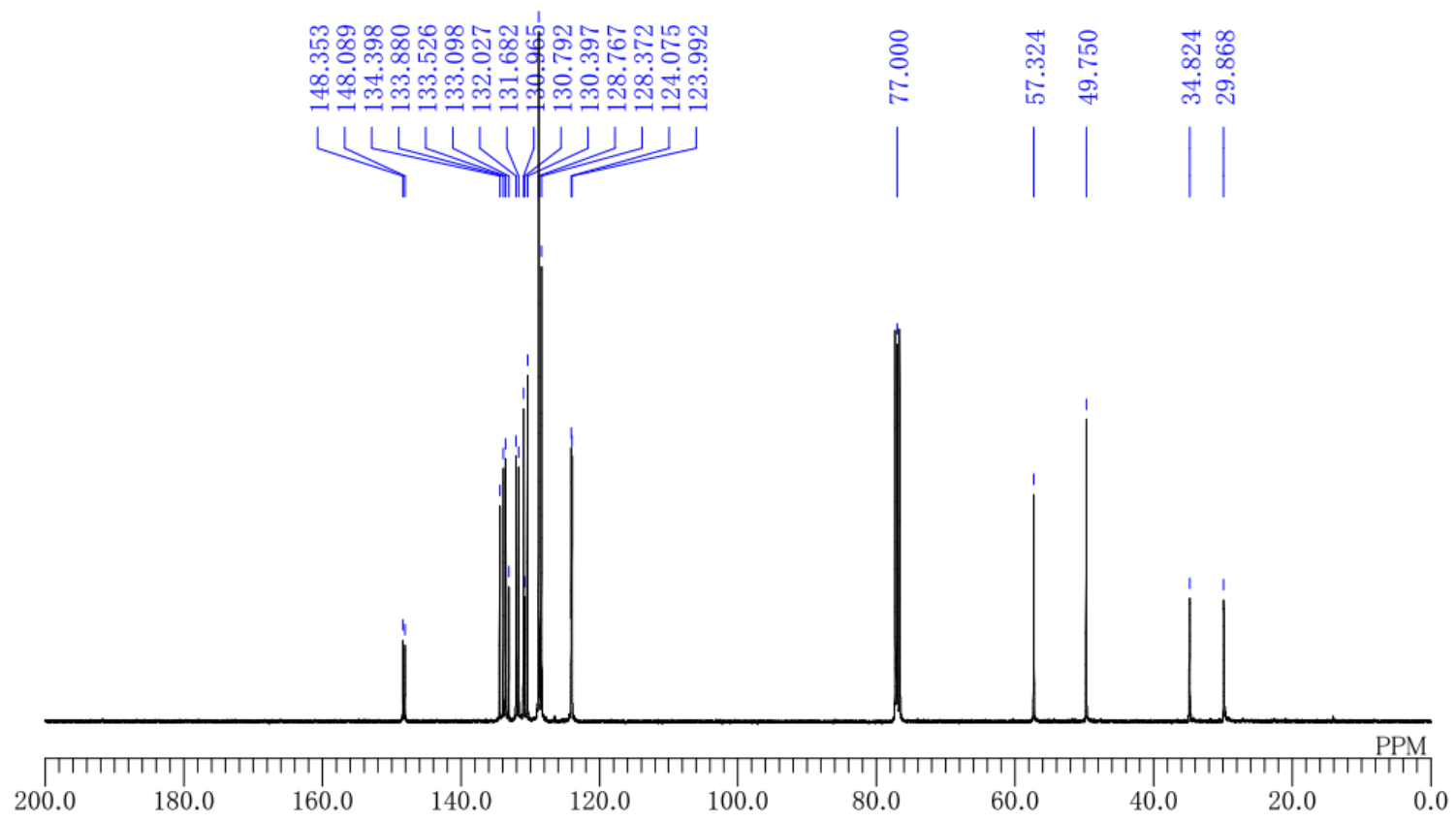


$\left(1 R^{*}, 2 R^{*}\right)$-2,3-dihydro-1H-indene-1,2-diaminium ditosylate (4ea)<smiles>N[C@@H]1Cc2ccccc2[C@H]1N</smiles>

${ }^{1} \mathrm{H}$ NMR (400 MHz, DMSO- $\left.d_{6}\right)$

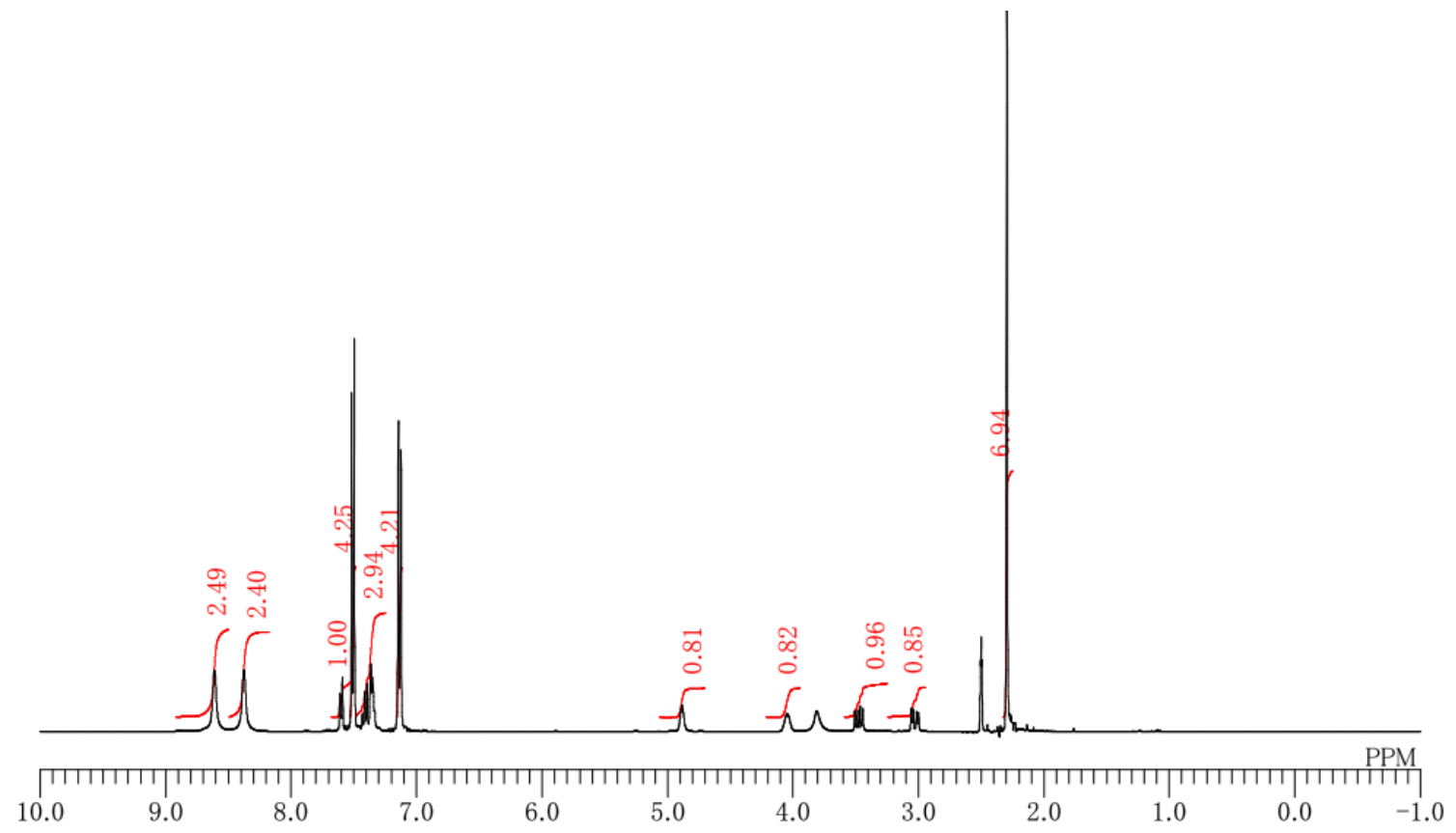

${ }^{13} \mathrm{C}$ NMR $\left(100 \mathrm{MHz}\right.$, DMSO- $\left.d_{6}\right)$

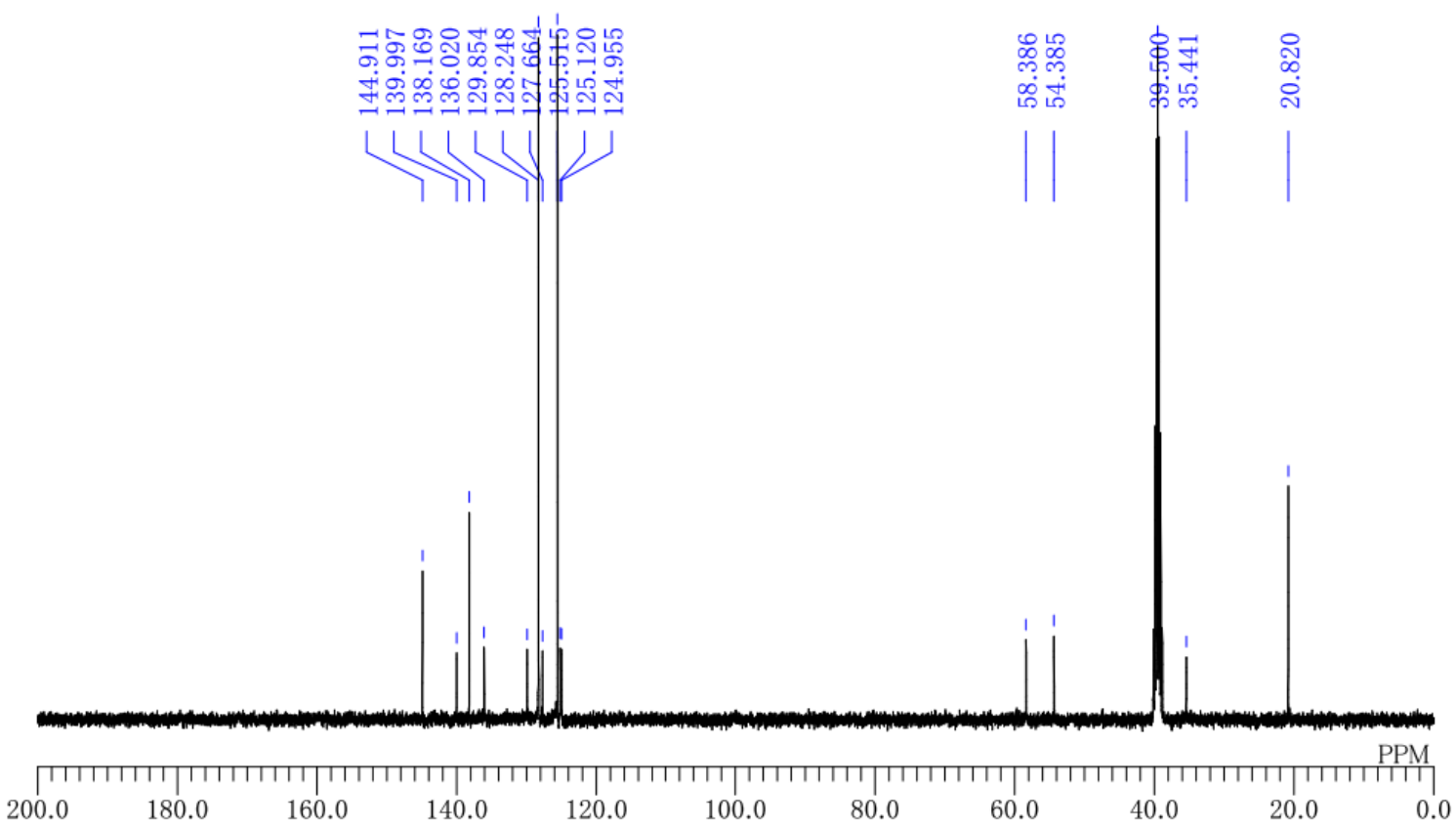


$\left(1 R^{*}, 2 S^{*}\right)-2,3-d i h y d r o-1 H$-indene-1,2-diaminium ditosylate (5ea)<smiles>NC1Cc2ccccc2C1N</smiles>

${ }^{1} \mathrm{H}$ NMR (400 MHz, DMSO- $\left.d_{6}\right)$

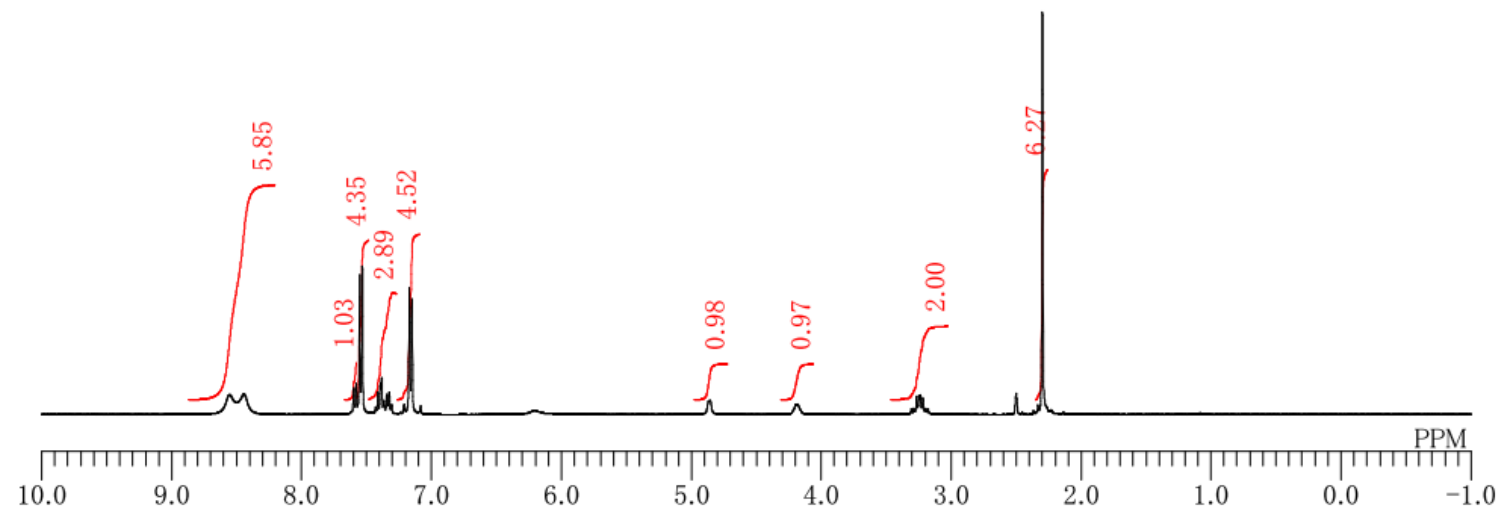

${ }^{13} \mathrm{C}$ NMR (100 MHz, DMSO- $\left.d_{6}\right)$

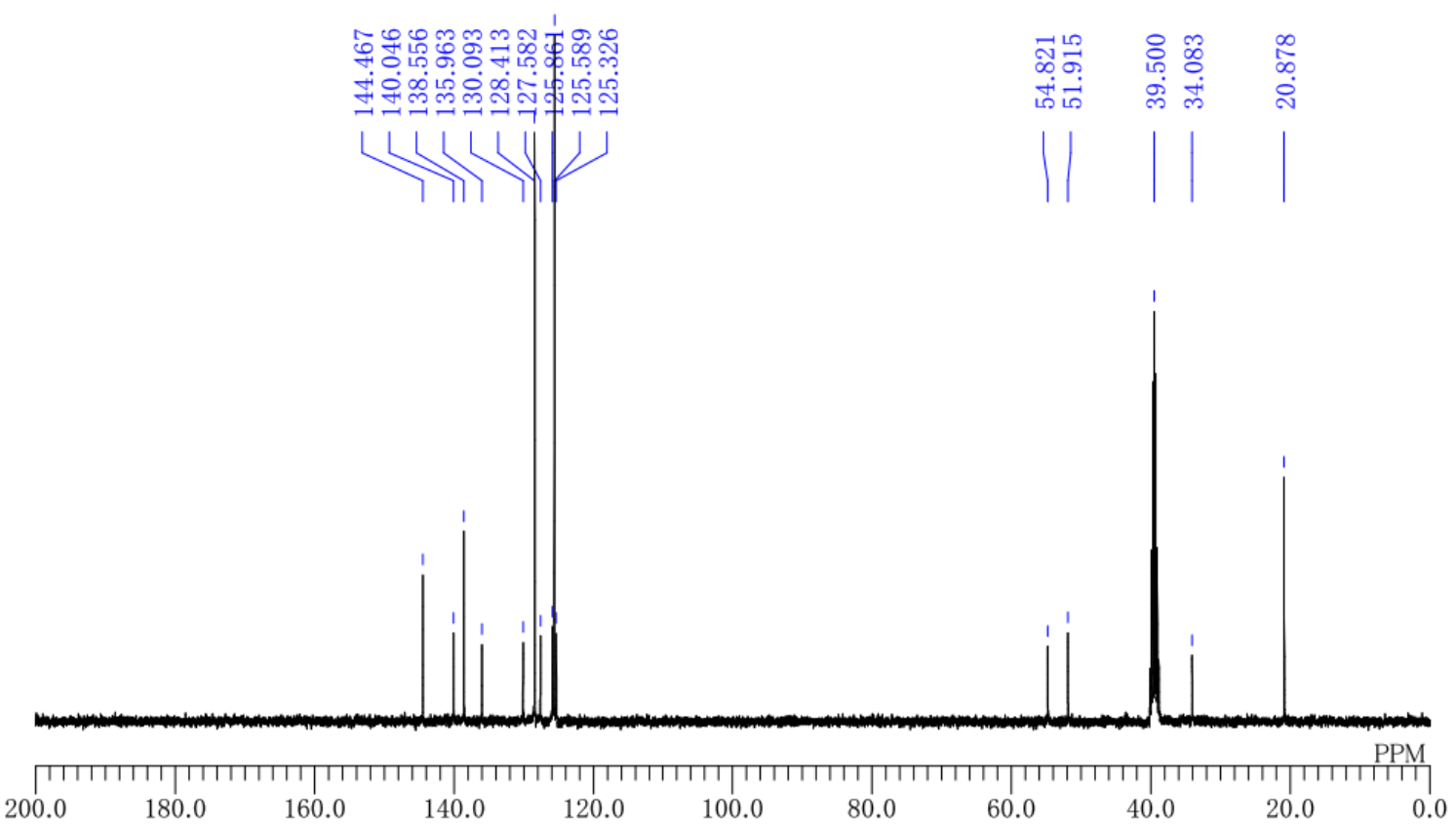


$\mathrm{N}$-allyl-4-methyl-benzenesulfonamide (1j)

Ts ${ }^{H}$<smiles>C=CC[AlH2]</smiles>

${ }^{1} \mathrm{H}$ NMR $\left(400 \mathrm{MHz}, \mathrm{CDCl}_{3}\right)$

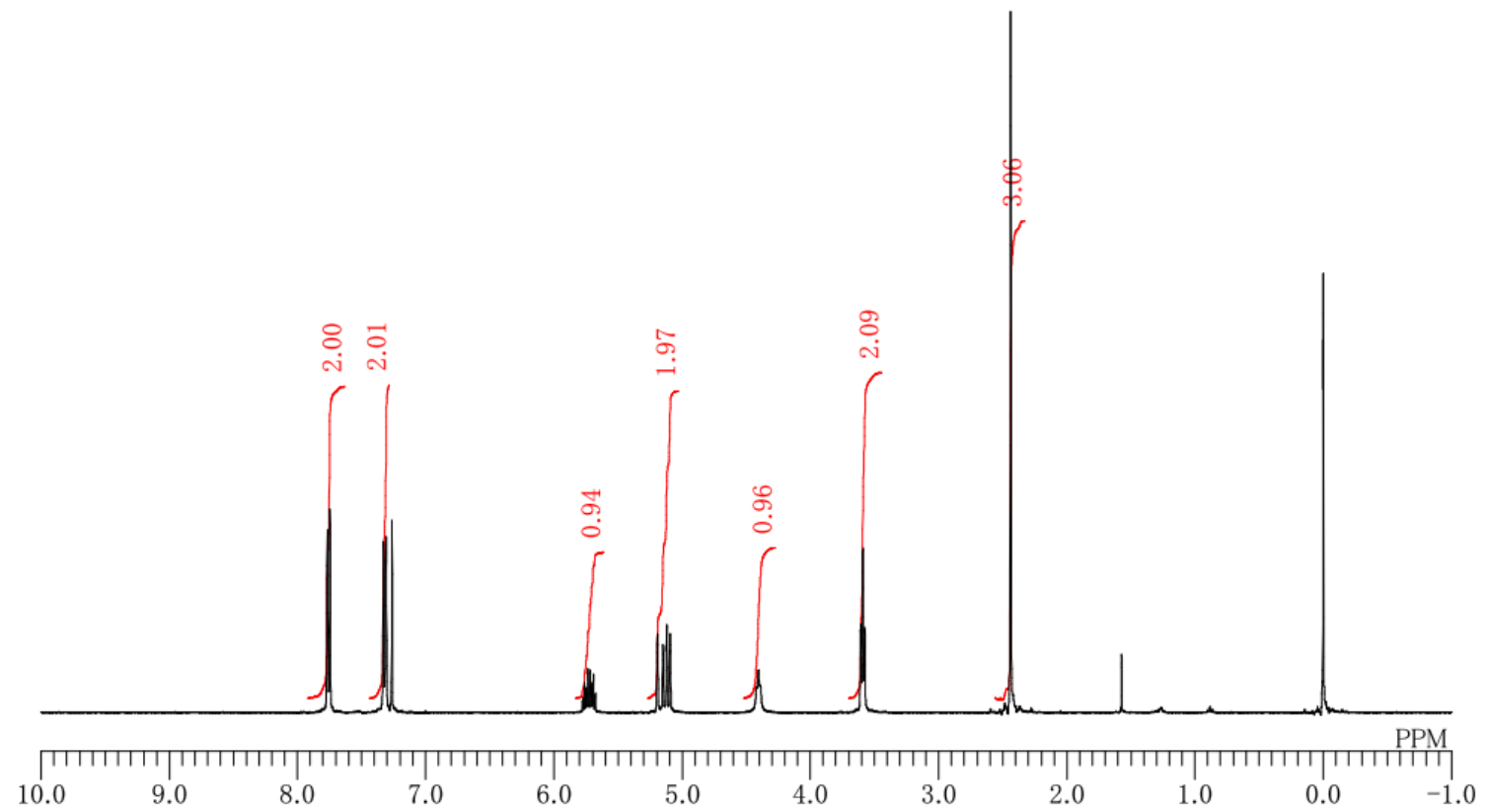

${ }^{13} \mathrm{C}$ NMR $\left(100 \mathrm{MHz}, \mathrm{CDCl}_{3}\right)$

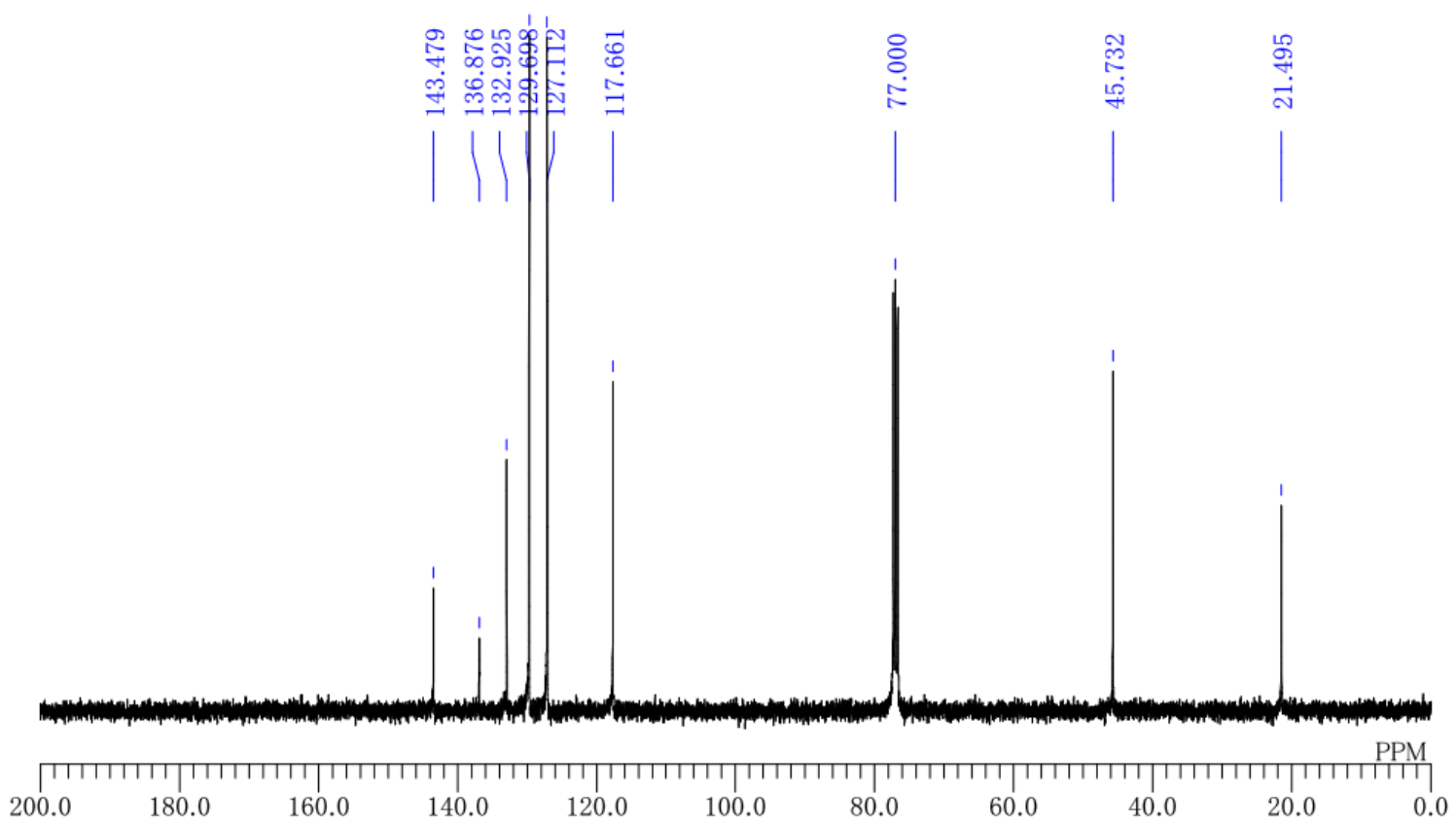




\section{3-vinylanisole (1p)}<smiles>C=Cc1cccc(OC)c1</smiles>

${ }^{1} \mathrm{H}$ NMR (400 MHz, $\mathrm{CDCl}_{3}$ )

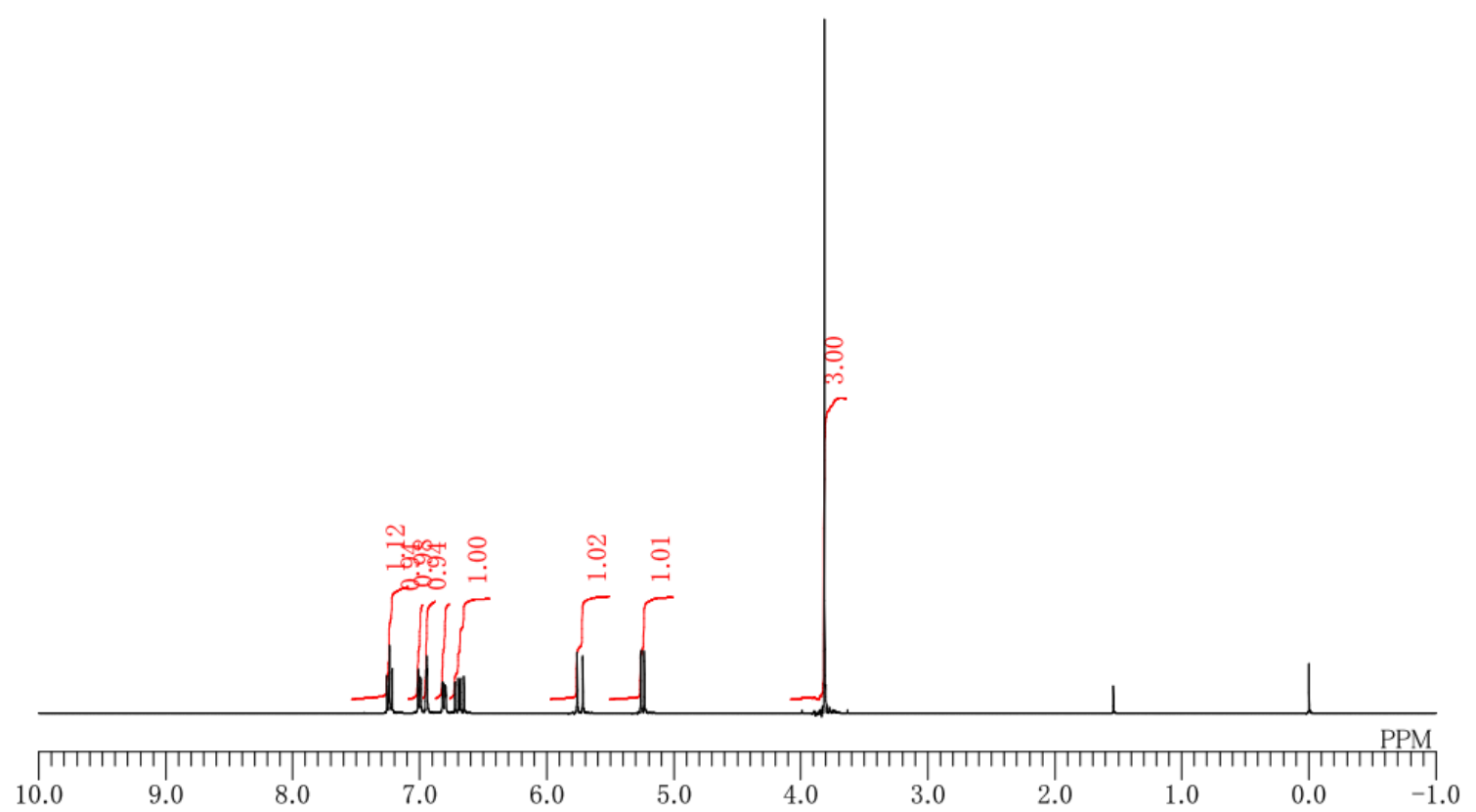

${ }^{13} \mathrm{C}$ NMR $\left(100 \mathrm{MHz}, \mathrm{CDCl}_{3}\right)$
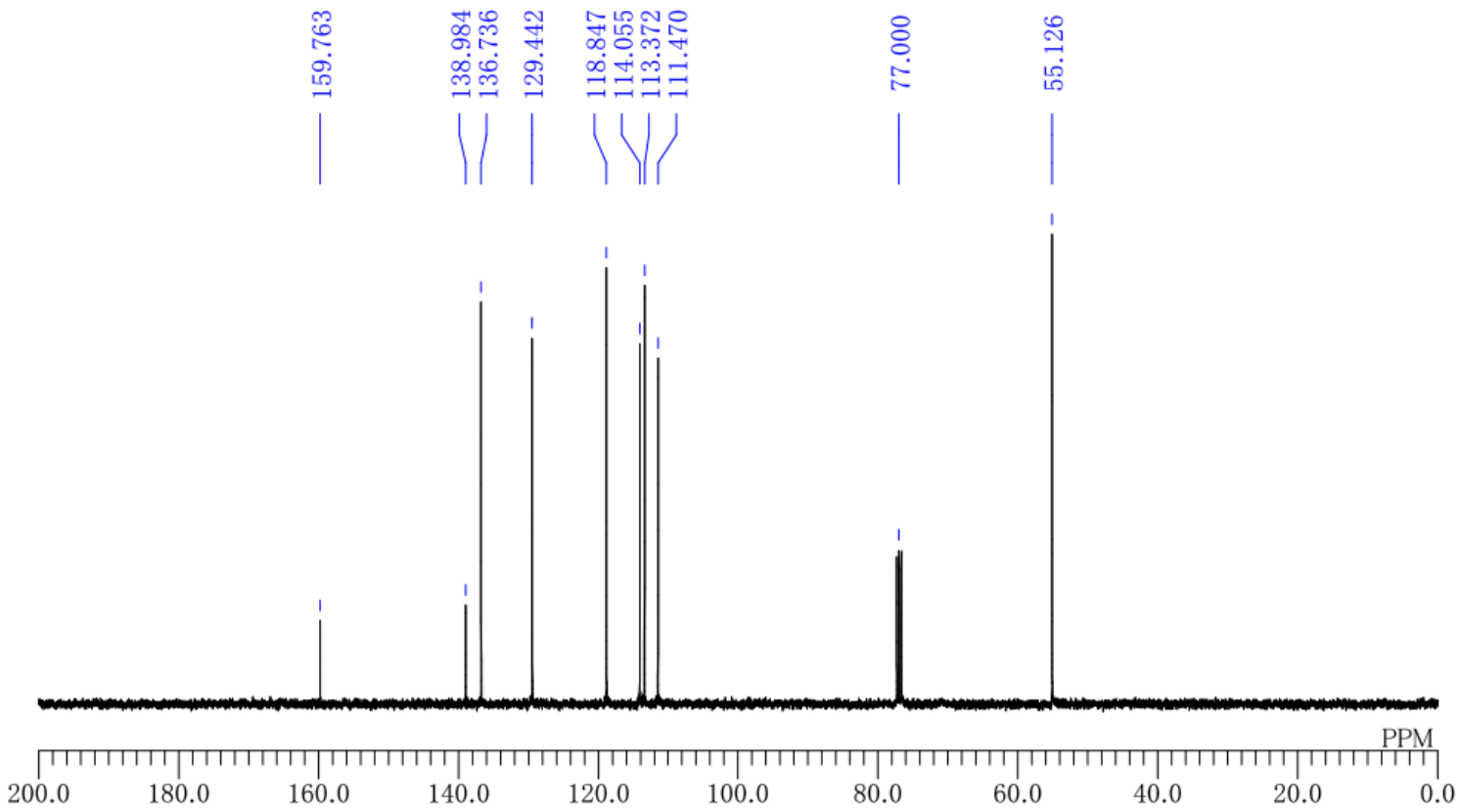
cis-(hex-3-en-1-yloxy)tert-butyldimethylsilane (2n)<smiles></smiles>

${ }^{1} \mathrm{H}$ NMR (400 MHz, $\mathrm{CDCl}_{3}$ )

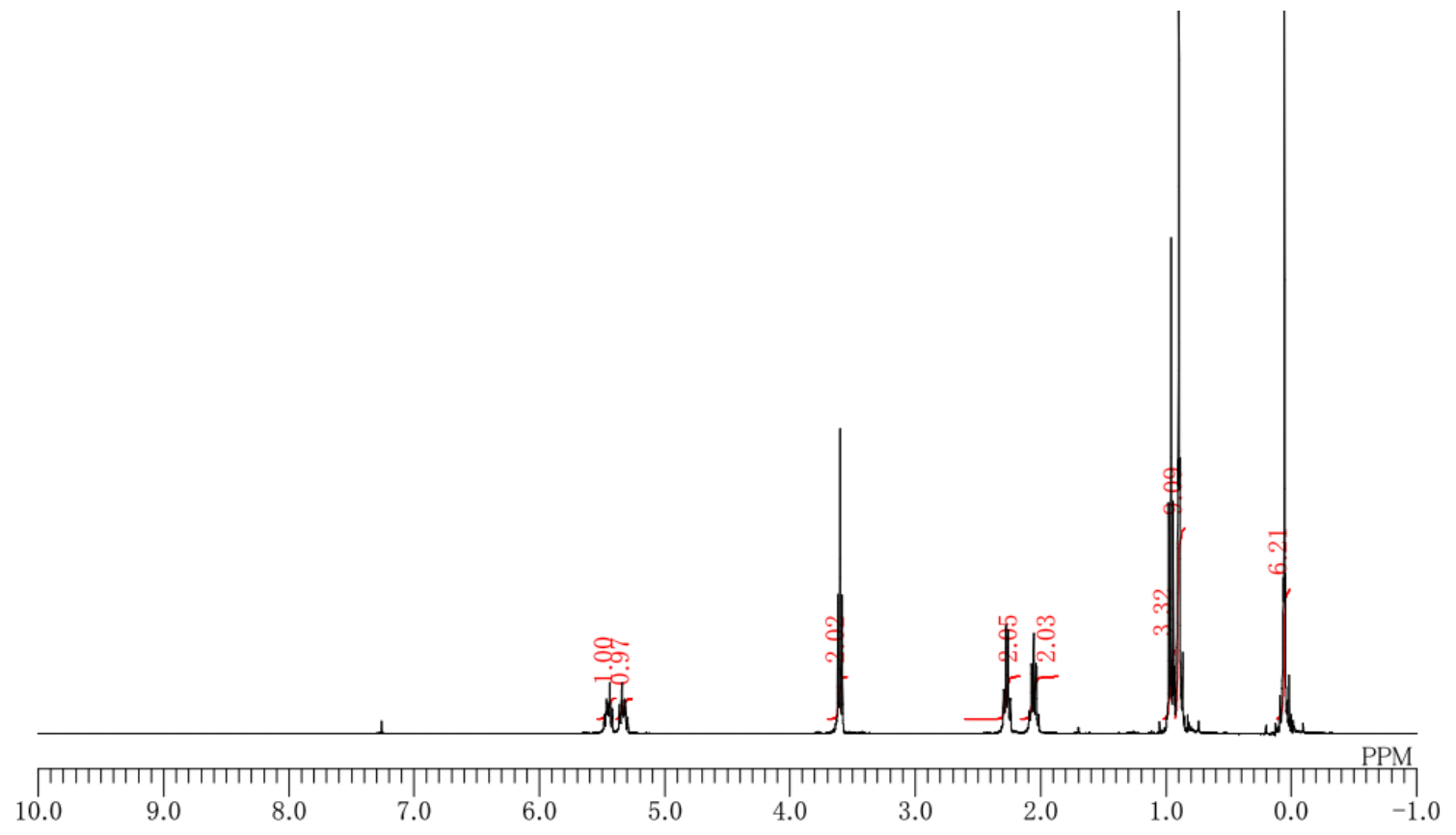

${ }^{13} \mathrm{C} \mathrm{NMR}\left(100 \mathrm{MHz}, \mathrm{CDCl}_{3}\right)$
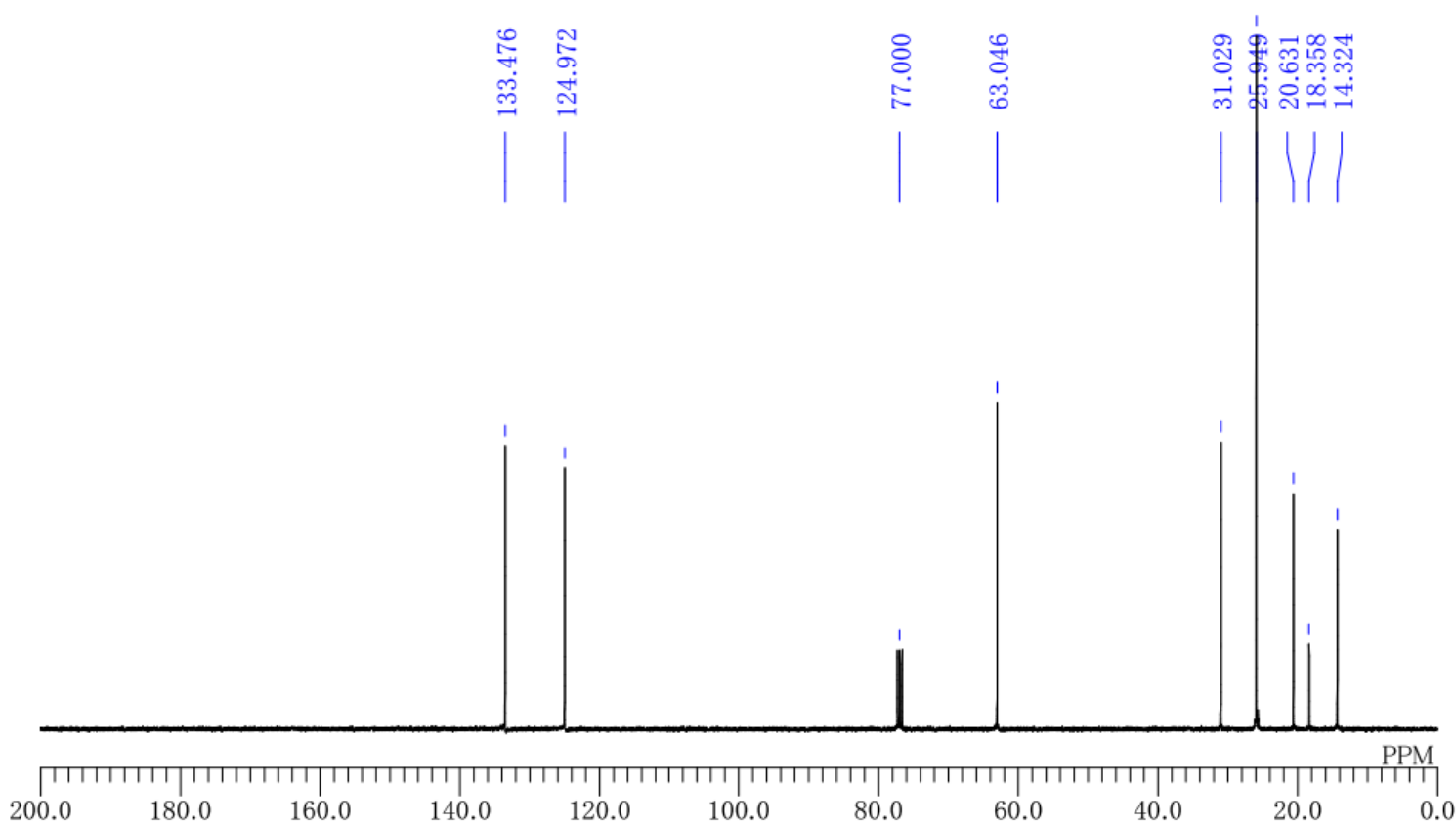
6-bromo-1 $H$-indene (2r)<smiles>Brc1ccc2c(c1)CC=C2</smiles>

${ }^{1} \mathrm{H}$ NMR $\left(400 \mathrm{MHz}, \mathrm{CDCl}_{3}\right)$

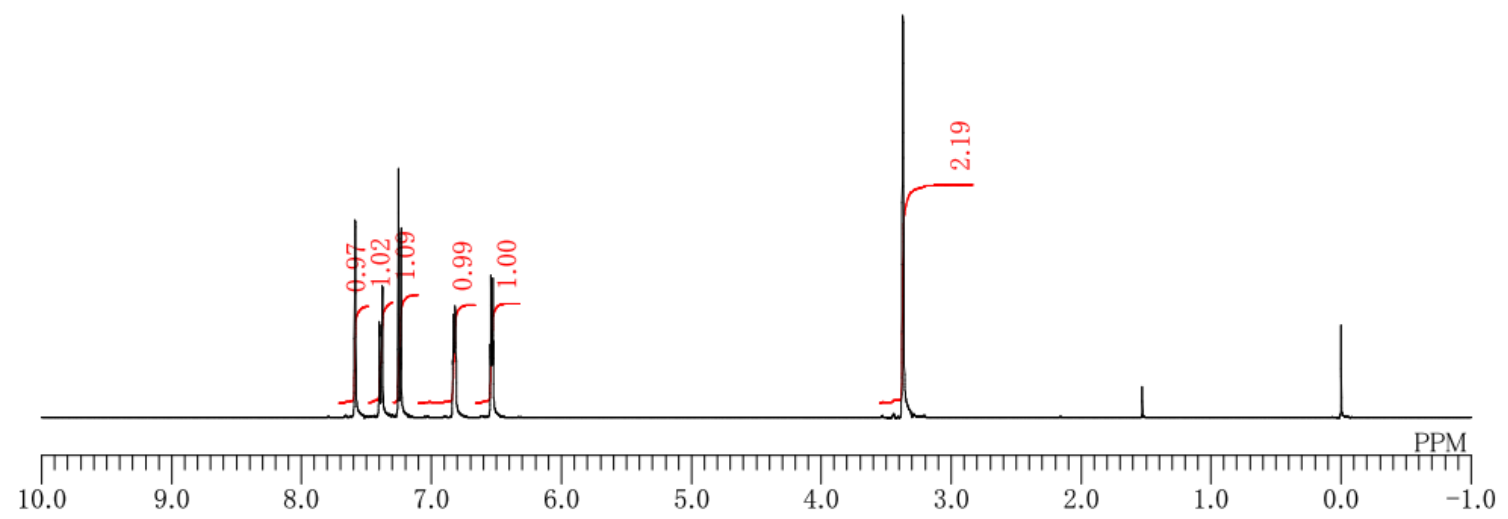

${ }^{13} \mathrm{C}$ NMR $\left(100 \mathrm{MHz}, \mathrm{CDCl}_{3}\right)$

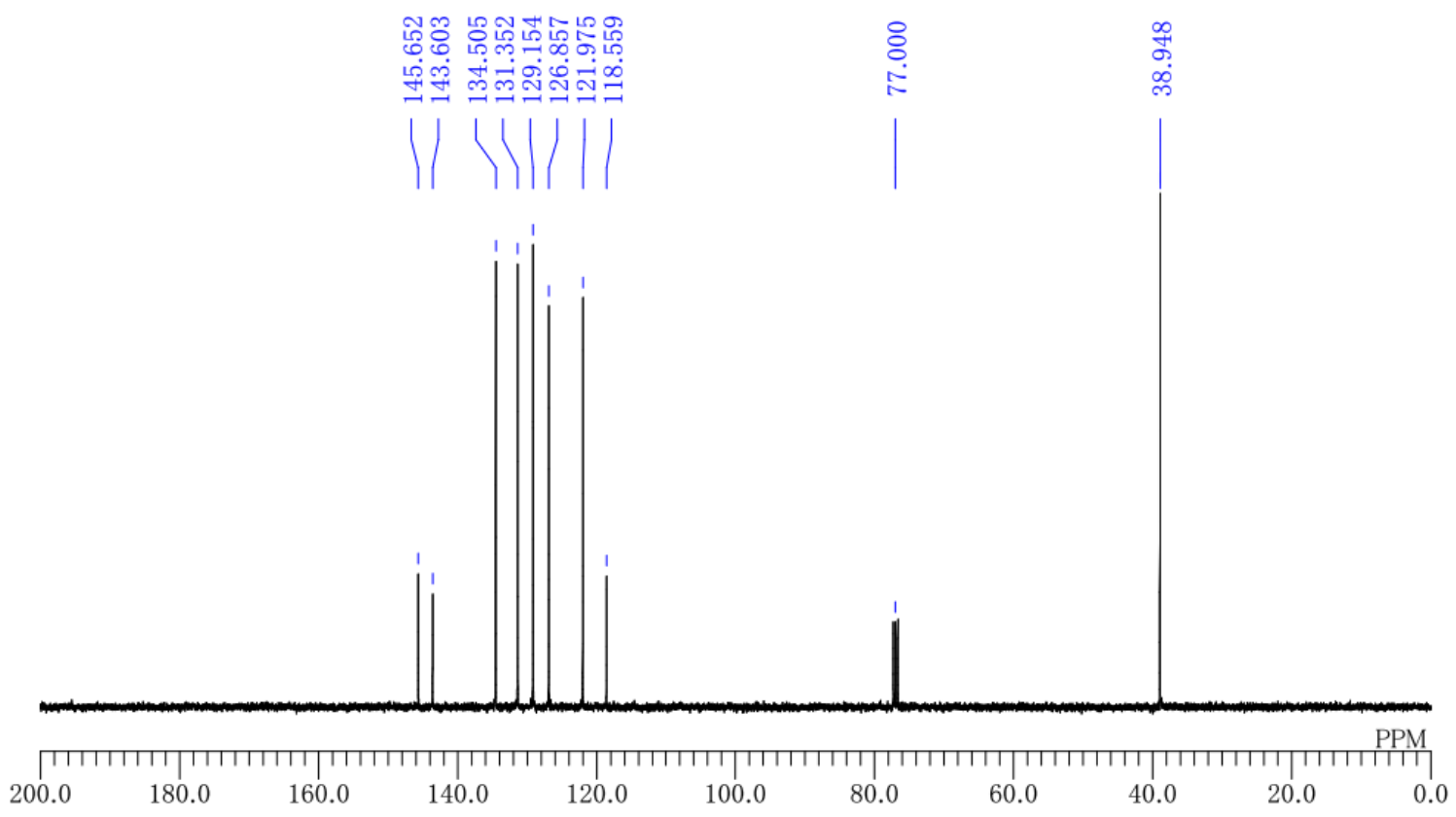


tert-butyl-1H-indole-1-carboxylate (2t)<smiles>CC(C)(C)OC(=O)n1ccc2ccccc21</smiles>

${ }^{1} \mathrm{H}$ NMR (400 MHz, $\left.\mathrm{CDCl}_{3}\right)$

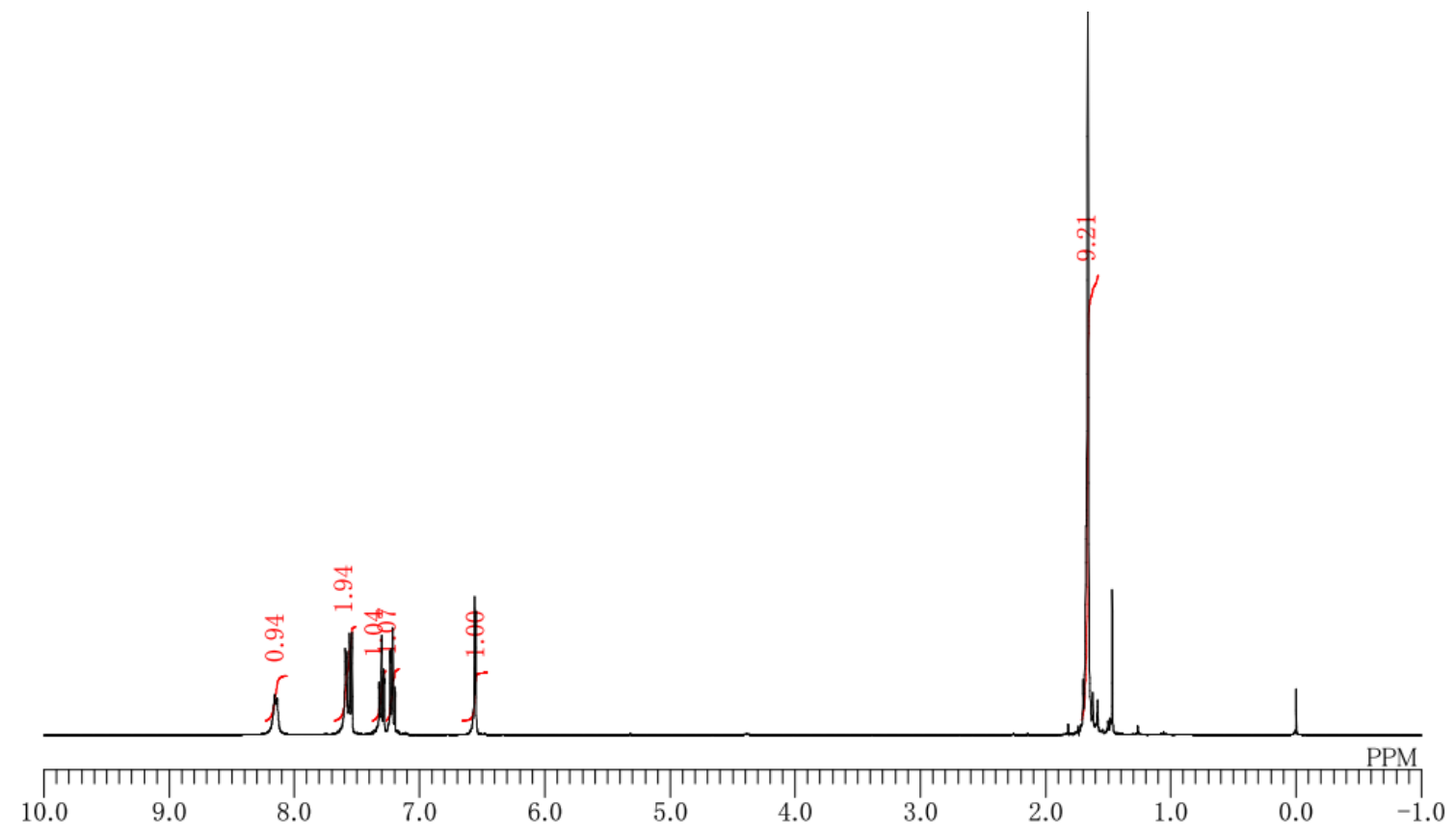

${ }^{13} \mathrm{C}$ NMR $\left(100 \mathrm{MHz}, \mathrm{CDCl}_{3}\right)$
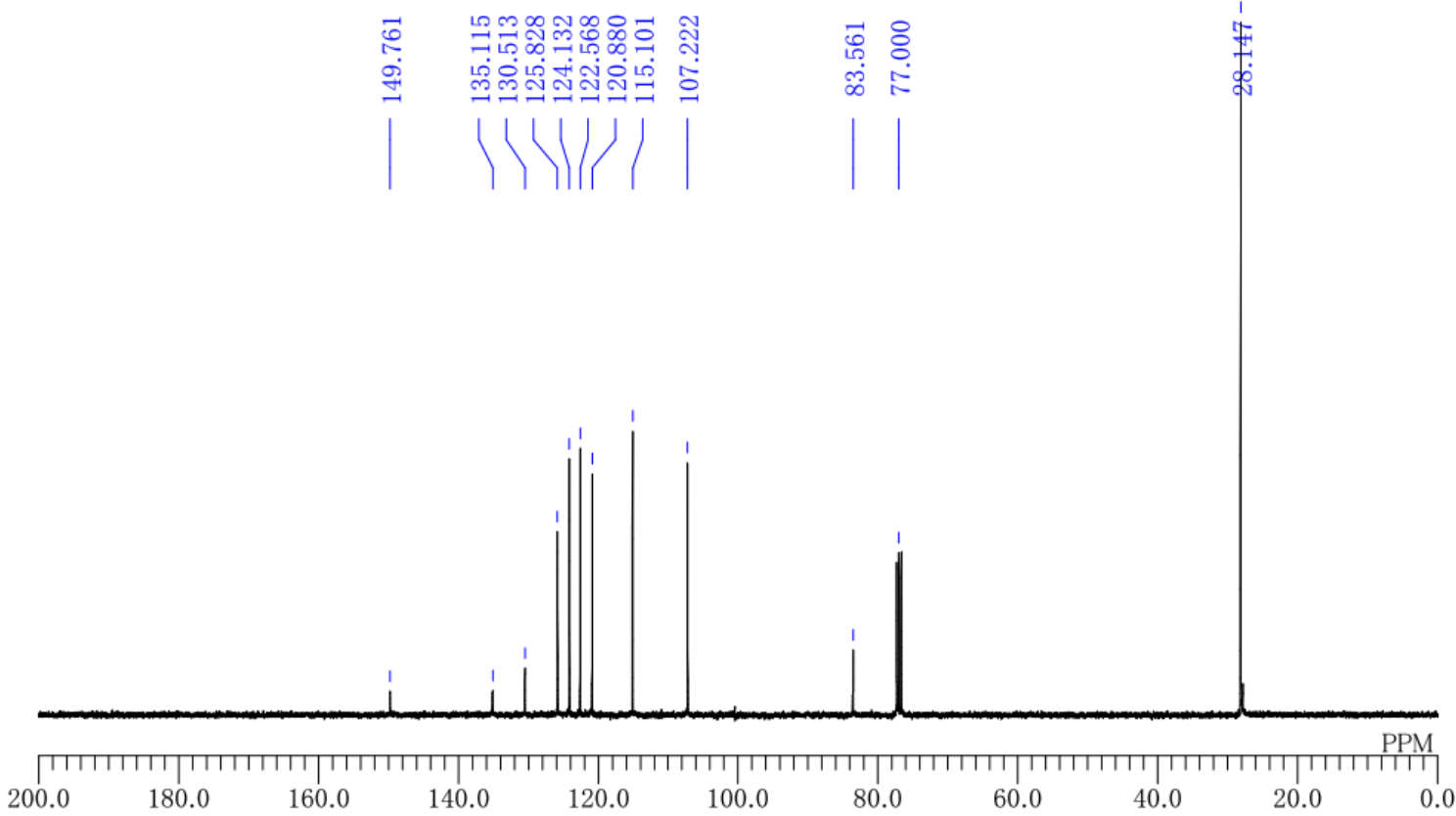
7-methoxy-1.2-dihydronaphthalene (2s)<smiles>COc1ccc2c(c1)CCC=C2</smiles>

${ }^{1} \mathrm{H}$ NMR $\left(400 \mathrm{MHz}, \mathrm{CDCl}_{3}\right)$

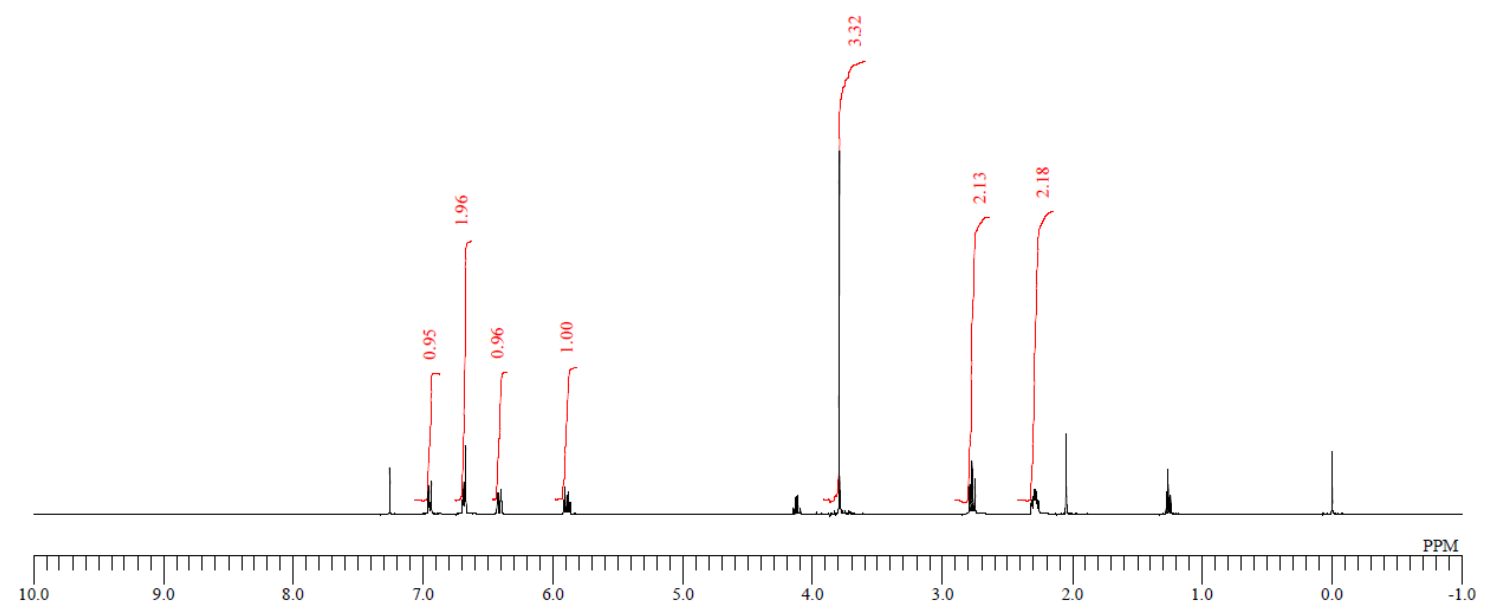

${ }^{13} \mathrm{C}$ NMR (100 MHz, $\left.\mathrm{CDCl}_{3}\right)$
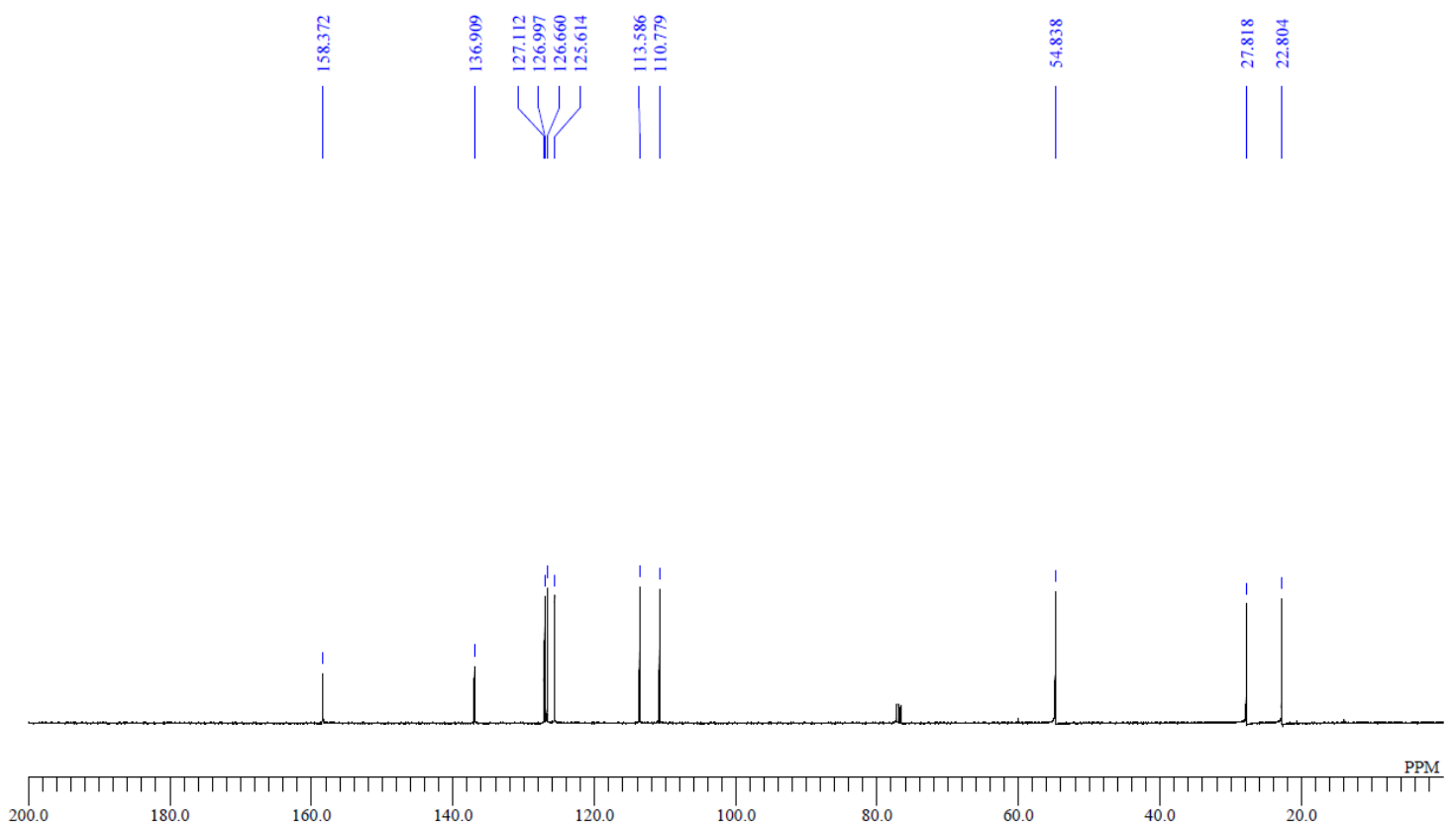
tert-butyl 5-methoxy-1 $H$-indole-1-carboxylate (2v)<smiles>COc1ccc2c(ccn2C(=O)OCc2ccccc2)c1</smiles>

${ }^{1} \mathrm{H}$ NMR $\left(400 \mathrm{MHz}, \mathrm{CDCl}_{3}\right)$

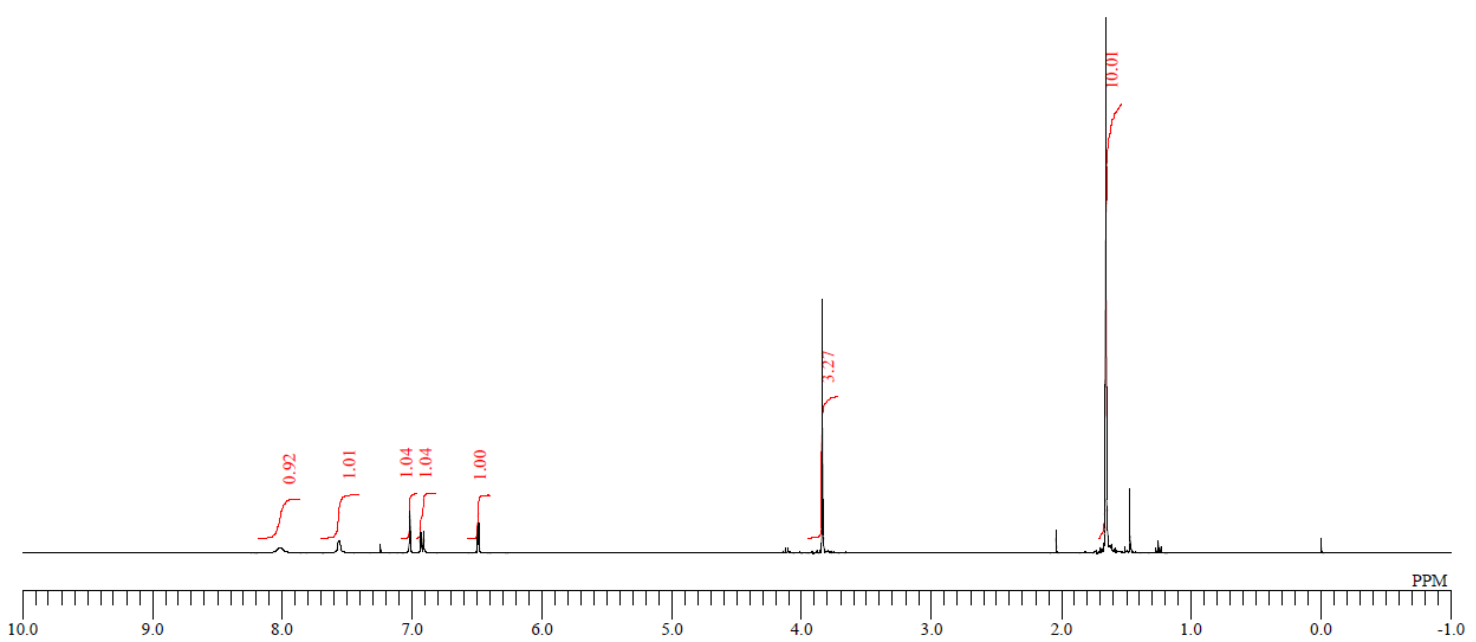

${ }^{13} \mathrm{C}$ NMR (100 MHz, $\mathrm{CDCl}_{3}$ )
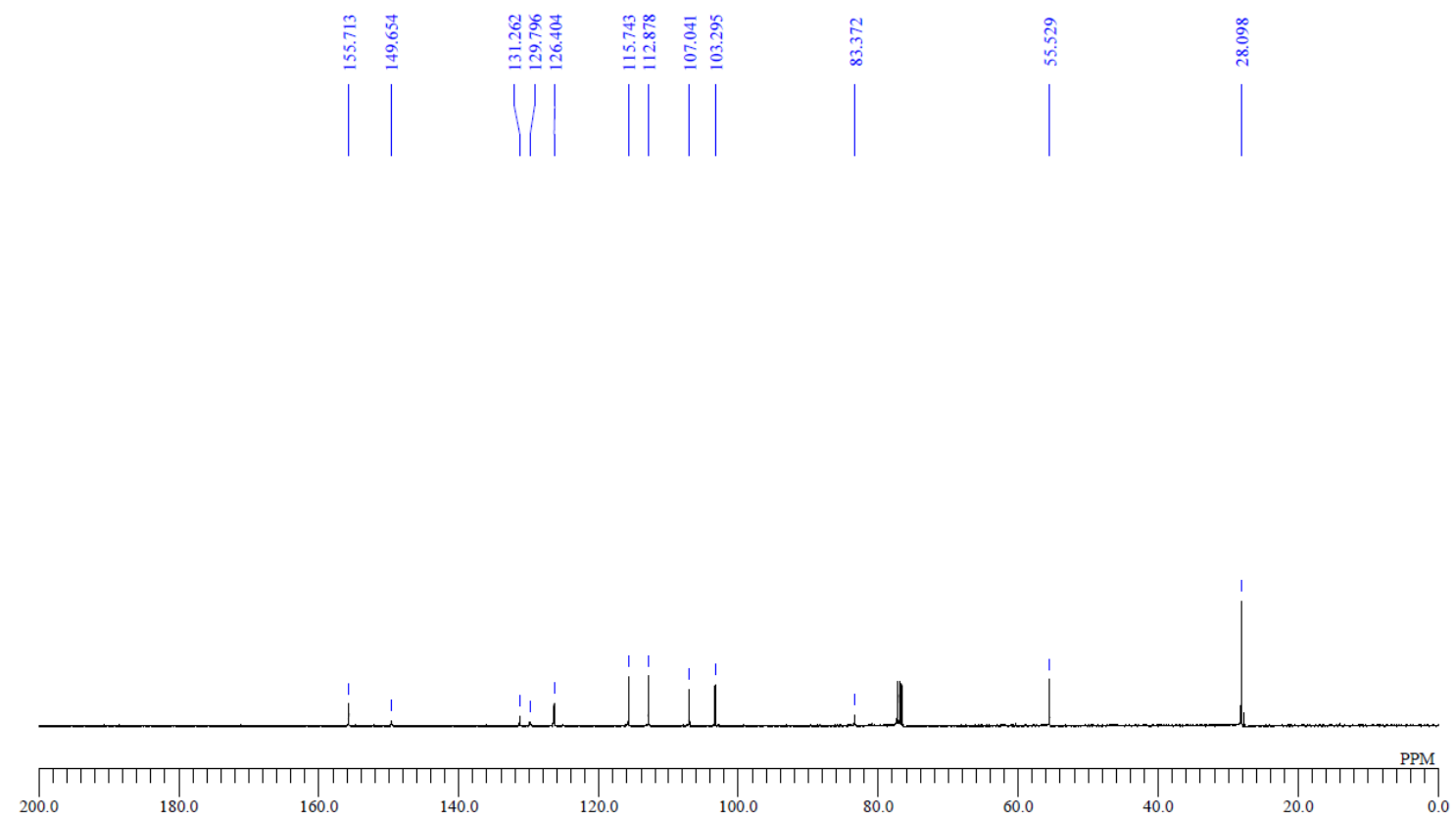
$N$-chloro- $N$-sodio-2-nitrobenzenesulfonamide dihydrate

$\mathrm{Na}$

$\mathrm{N}-\mathrm{Ns} \cdot 2 \mathrm{H}_{2} \mathrm{O}$

$\mathrm{Cl}^{\prime}$

${ }^{1} \mathrm{H}$ NMR (400 MHz, DMSO- $\left.d_{6}\right)$

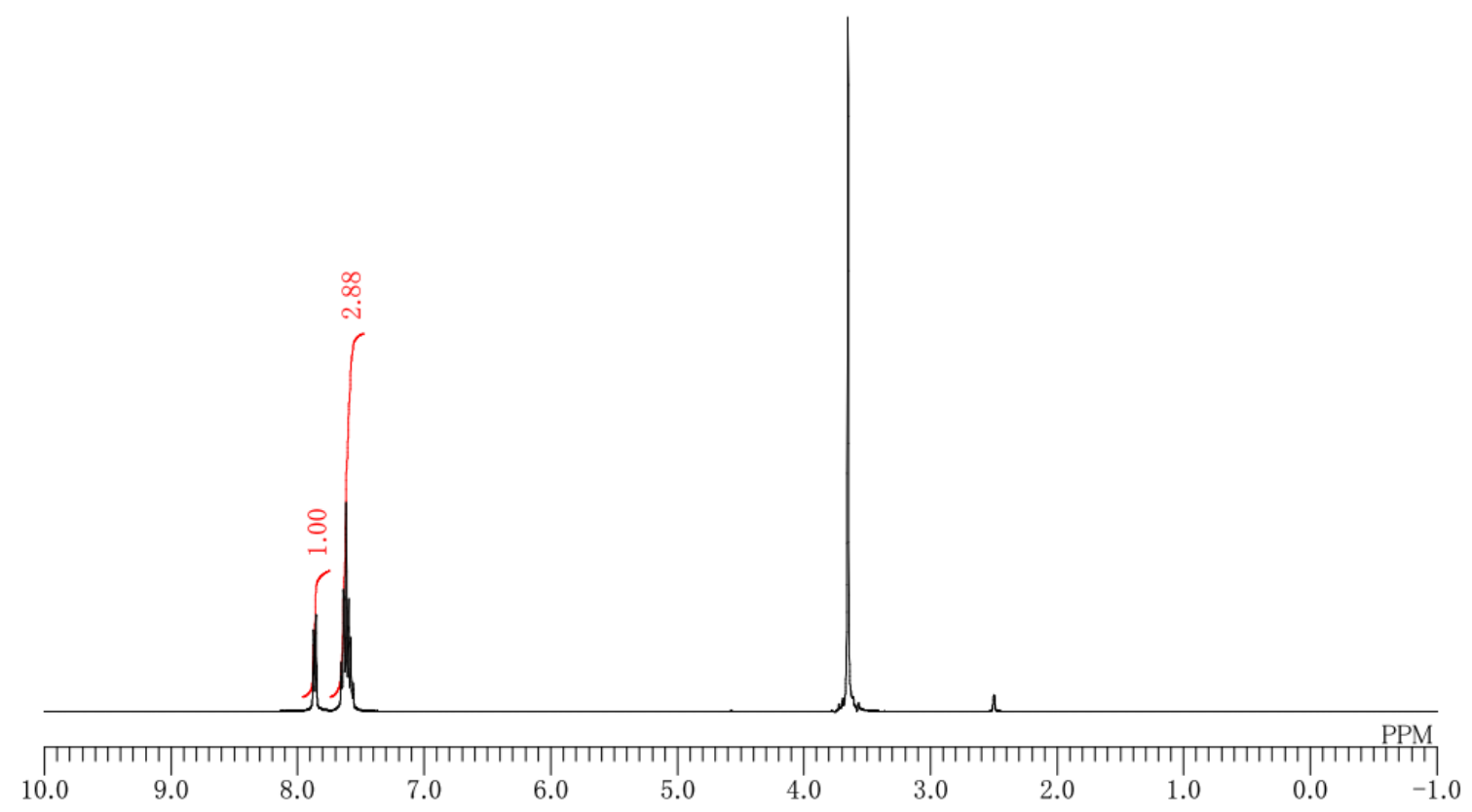

${ }^{13} \mathrm{C}$ NMR (100 MHz, DMSO- $\left.d_{6}\right)$

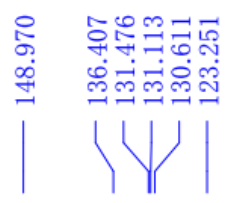

울.

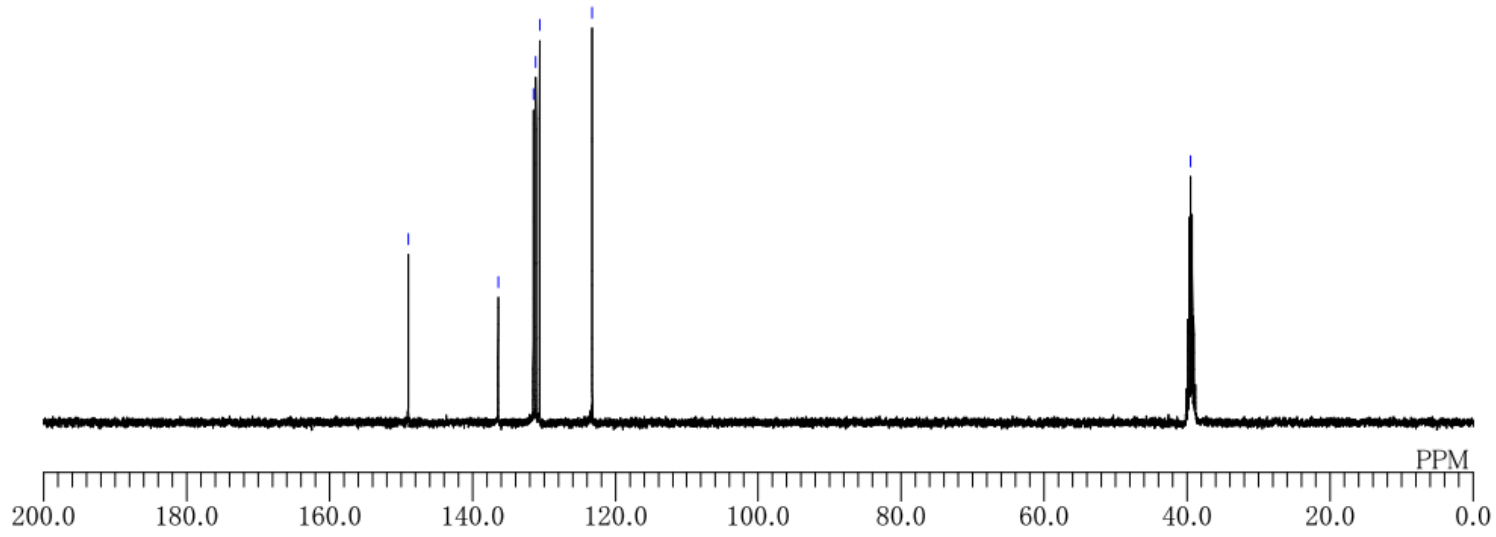




\section{$N, N^{\prime}$-bis(tert-butoxycarbonyl)sulfamide (BBS)}

Boc $\mathrm{H}^{\mathrm{O}} \mathrm{H}^{\mathrm{O}}, \mathrm{S}^{\prime \prime} \mathrm{H}^{-\mathrm{O}}$

${ }^{1} \mathrm{H}$ NMR (400 MHz, DMSO-d $)$

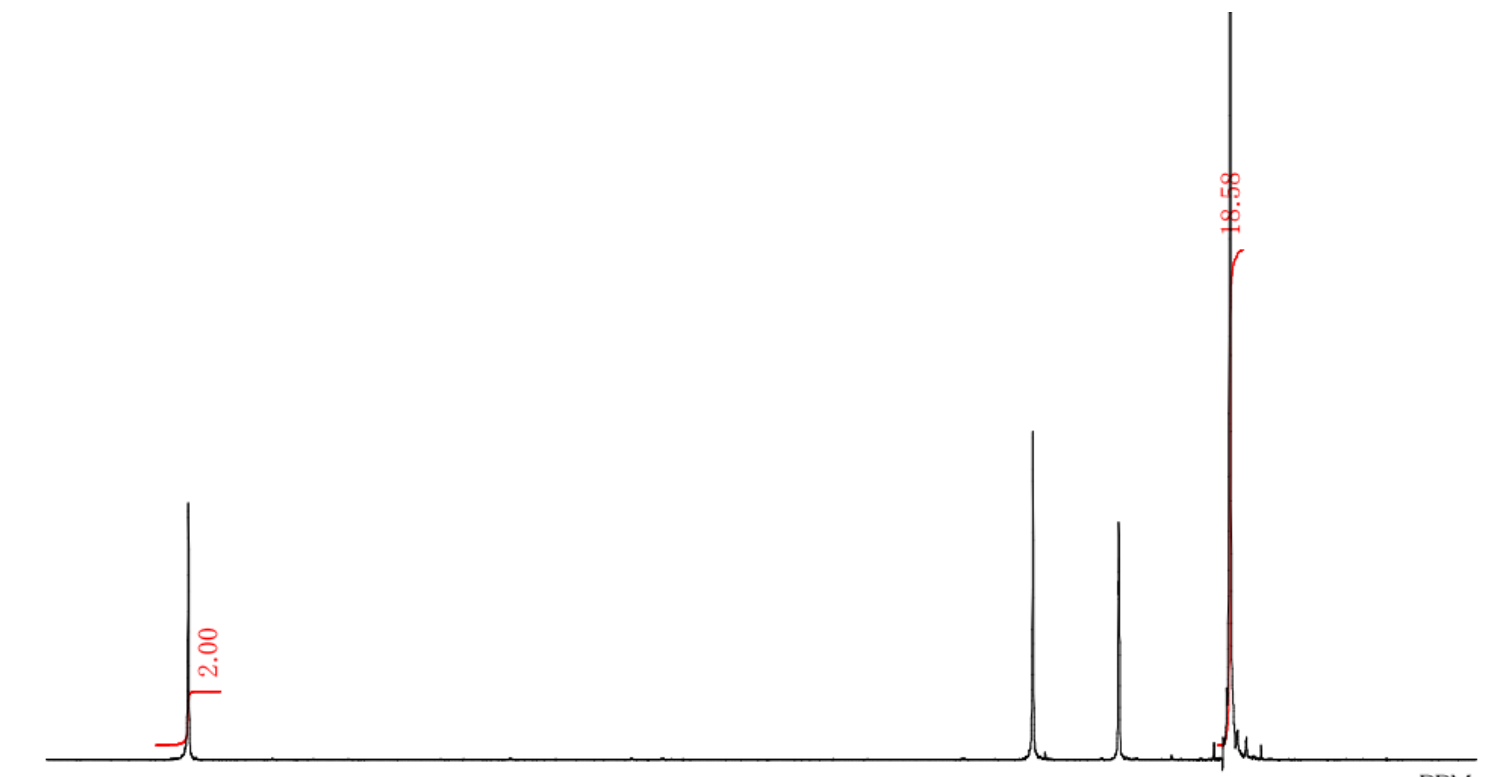

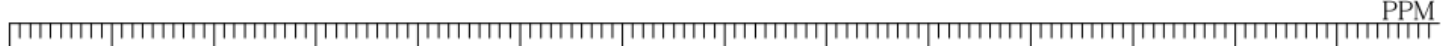

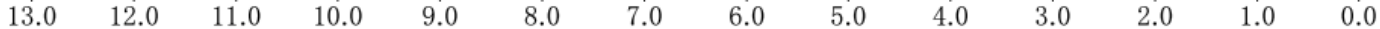

${ }^{13} \mathrm{C}$ NMR $\left(100 \mathrm{MHz}, \mathrm{DMSO}-d_{6}\right)$

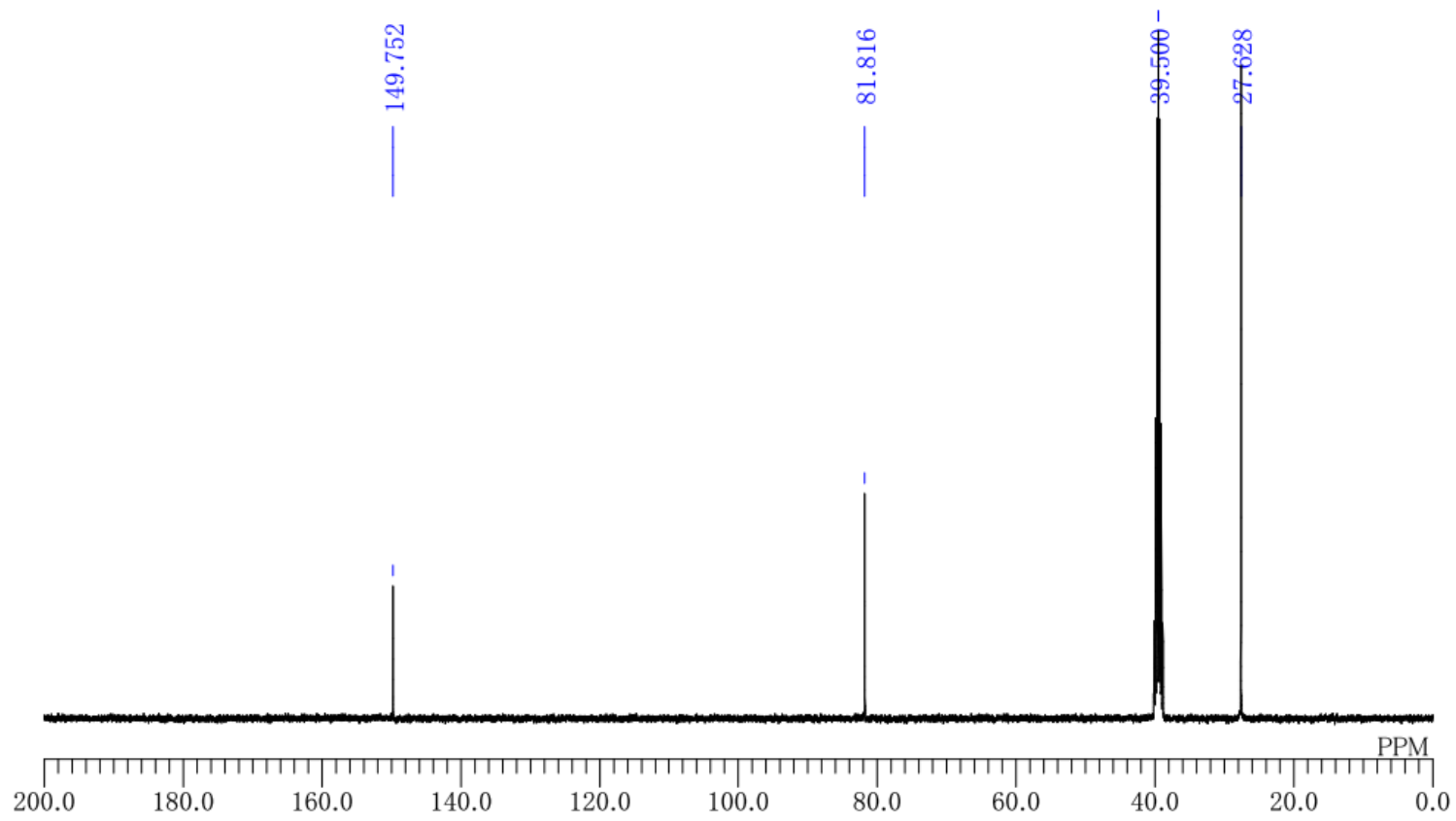


sodium(tert-butoxycarbonyl)( $N$-(tert-butoxycarbonyl)- $N$-chlorosulfamoyl)amide BBS)

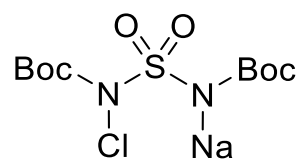

${ }^{1} \mathrm{H}$ NMR $\left(400 \mathrm{MHz}\right.$, Acetone- $\left.d_{6}\right)$

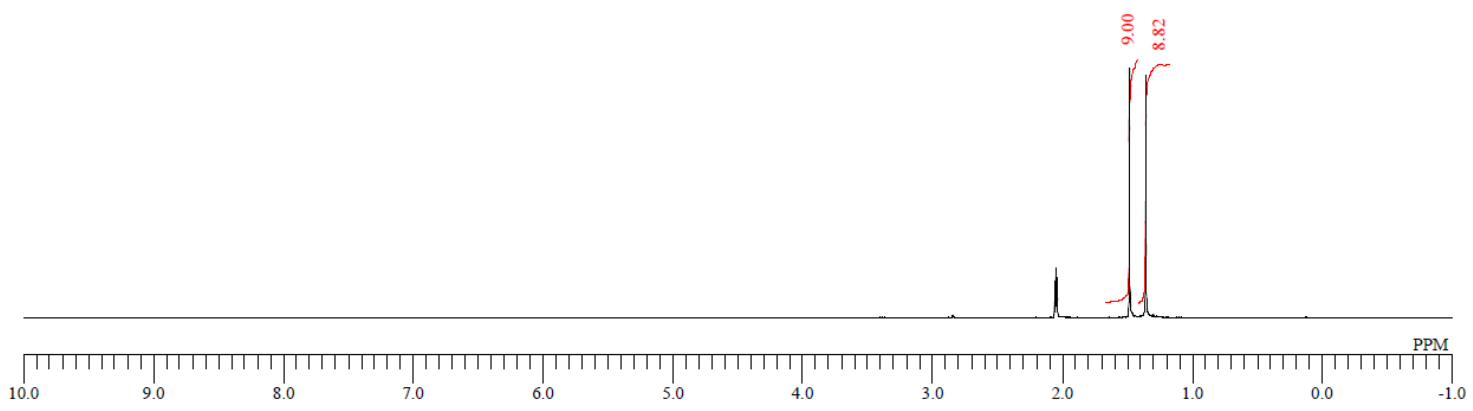




\section{References}

1. Ando, T.; Kano, D.; Minakata, S.; Ryu, I.; Komatsu, M. Iodine-catalyzed aziridination of alkenes using chloramine-T as a nitrogen source. Tetrahedron 1988, 54, 13485-13494.

2. Yuan, Y.-A.; Lu, D,-F.; Chen, Y.-R.; Xu, H. Iron-catalyzed direct diazidation for a broad range of olefins. Angew. Chem. Int. Ed. 2016, 53, 534-538.

3. Park, H.; Hong, Y. L.; Kim, Y. B.; Choi, T. L. Synthesis of small and large fused bicyclic compounds by tandem dienyne ring-closing metathesis. Org. Lett. 2010, 12, 3442-3445 .

4. Jones, A. S.; Paliga, J. F.; Greenhalgh, M. D.; Quibell, J. M.; Steven, A.; Thomas, S. P. Broad scope hydrofunctionalization of styrene derivatives using iron-catalyzed hydromagnesiation. Org. Lett. 2014, 16, 5964-5967.

5. Jat, J. L.; Paudyal, M. P.; Gao, H.; Xu, Q. L.; Yousufuddin, M.; Devarajan, D.; Ess, D. H.; Kürti, L.; Falck, J. R. Direct stereospecific synthesis of unprotected N-H and N-Me aziridines from olefins. Science 2014, 343, 61-65.

6. Constanzo, M. J. et al., patent application WO2009067202 (2009).

7. Barcellos, J. C. F.; Borges, B. H. F.; Mendes, J. A.; Ceron, M. C.; Buarque, C. D.; Dias, A. G.; Costa, P. R. R. Synthesis of 11a- $N$-arylsulfonyl-5-carbapterocarpans (tetrahydro$5 H$-benzo $[a]$ carbazoles) by azaarylation of dihydronaphthalenes with $o$-iodo- $N$ (arylsulfonyl)anilines in poly(ethylene glycol). Synthesis 2015, 47, 3013-3019.

8. Chen, M.; Huang, Z. T.; Zheng, Q. Y. Visible light-induced 3-sulfenylation of $N$ methylindoles with arylsulfonyl chlorides. Chem. Commun. 2012, 48, 11686-11688.

9. Caramenti, P.; Nandi, R. K.; Waser, J. Metal-free oxidative cross coupling of indoles with electron-rich (hetero)arenes. Chem. Eur. J. 2018, 24, 10049-10053. 